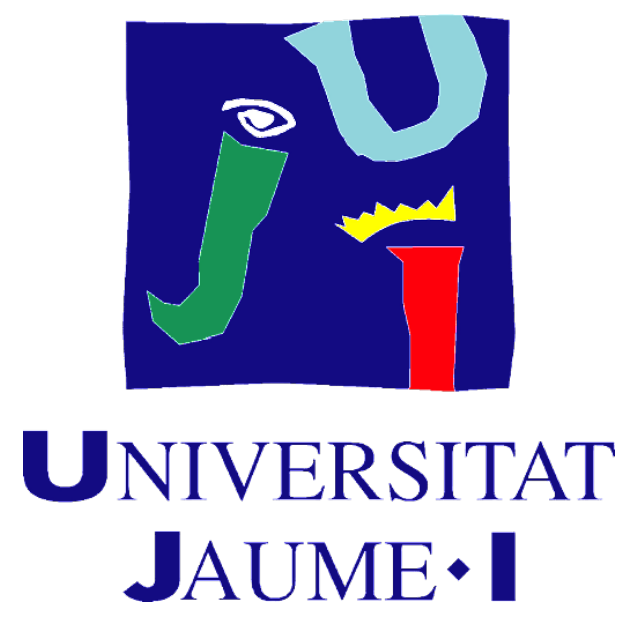

\title{
Making biosignals available
}

\author{
Doctoral candidate \\ Miquel Alfaras Espinàs
}

Supervisors: $\quad$ Dr. Hugo Gamboa (PLUX Wireless Biosignals S.A.) Dr. Angel Pascual del Pobil (Universitat Jaume I)

Submission date: July 2021

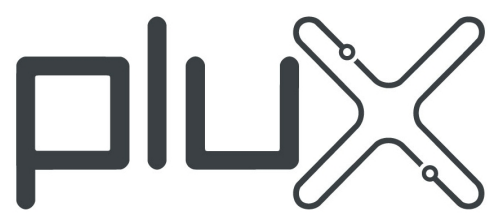




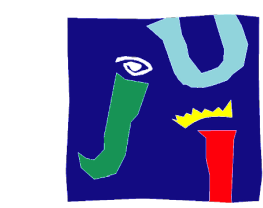

UNIVERSITAT

JAUME• I

Programme: Doctoral Programme in Computer Science Centre: Doctoral School of Universitat Jaume I

Thesis submitted by Miquel Alfaras Espinàs in fulfilment of the requirements for the degree of Doctor of Philosophy

Signatures:
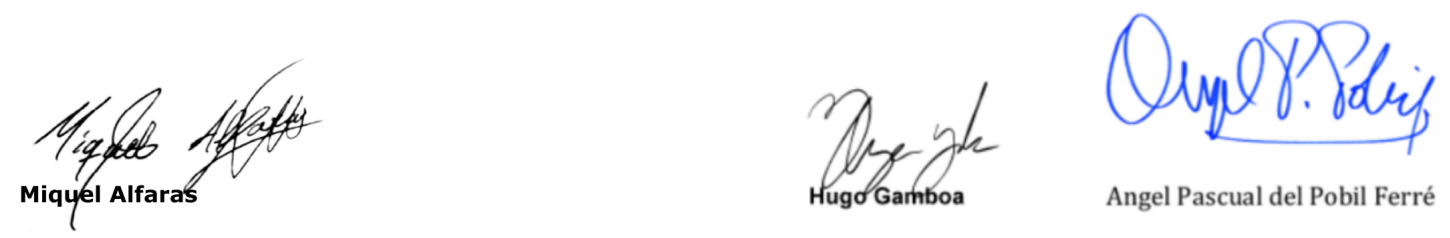

Castelló de la Plana, July 2021 


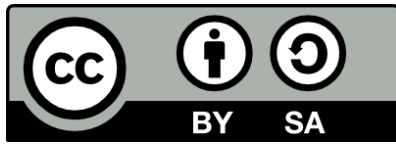

This work is licensed under CC BY-SA terms (Attribution-ShareAlike 2.0 Generic CC BY-SA 2.0)

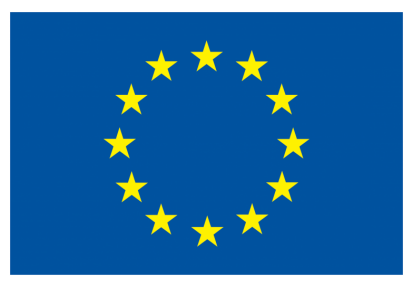

This research is funded by the European Commission within the Marie Skłodowska-Curie Actions (ITN-ETN). Grant Agreement num. 722022 AffecTech - H2020-MSCA-ITN-2016.

This thesis has been carried out under the support of an employment contract awarded at PLUX S.A.(Portugal) 2017-2020 within the European AffecTech ITN framework 



\section{Acknowledgements}

A PhD is a significant endeavour to push science and technology progress, with novel, unprecedented research fronts. A 3 year journey! 3+ years, actually. Marie Skłodowska Curie is a big name - one I was (and will be) proud to carry with honour. No wonder the flagship EU training frameworks are named after the physicist. That who best strived to feed her science hunger and her daughters too, opening a world that was never to be closed.

No matter how hard I try, I am likely missing some of the many people to thank for my doctoral path being a reality. First, to my ever-growing family, two to four, aquells més propers $i$ aquells més Ilunyans també, for the unconditional support and reliance. If living up to the expectations, part of the researcher in me comes from great glimmers of science in Granada discussions, long hours of physicists messing with other disciplines at IFISC Mallorca - such as my take at machine learning and biosignal processing-, and a recurrent curiosity I have not abandoned ever since, so that I can sneak peek into other fields. To those who believed in me, from algorithm optimisation to business intelligence leads required to fund a start-up, many thanks. I certainly learnt. To those who did not believe in me too, or did not see what I brought to the table. Failure - underestimated and overlooked failure - taught me more than success. Psychologists challenging science possibilities, taking technology to their limits, or our attempts to come up with novel audio-physiologic links, among many. For all those times I learnt in bursts. Those times I was allowed to be myself, hardheaded if needed, or letting me dream high, and those times doors were shut, me being brought down. A European PhD was for me a demanding challenge, making it into a position hard-fought by many, having an eye for tech transfer in the private side. First scene, thesis framework, supervisor, topic and being officially introduced at the company. "Talhado para mim, mal o conheci, eu achei-o desse modo. Logo pude perceber o fado que ia ter... " But honestly, many are to be thanked for it. First, to the supervisors, for opening the doors of their homes, getting close, in Lisbon, with a vast world built up around physiology tech; and in Castelló, for a nothing-to-lose opportunity that brought me closer to the Spanish academia flair - the good and the bad - while exchanging thoughts with roboticists (all RoblnLab, IRS and EMAROs), bringing a much needed writing window. The AffecTech crowd deserves a high place in the acknowledgements. Mentors in the battlefield and ESRs, above all. To the survivors, to the pioneers whose strength and supervisory team led to defend on time, and to the ones struggling to get through. Shadi, Claudia... M.Umair, my most relevant discovery alongside my adoptive $\mathrm{HCl}$ parents. Charles, for laying bridges. Javier, for having a truly interdisciplinary spirit. To Pavel, Andrea, Chengcheng, Niaz, Dionne, Camille, Hristo, Desirèe. All the places that welcomed us. The resulting language mixture I was always eager to grasp. To all those who welcomed us at Boğaziçi, Grinda, Milano, al LABPSITEC, and in InfoLab21. And to William, the travelling companion in Lisbonshire who, to me, appeared as infused by Grierson-Fiebrink teachings, exemplifying creative computing on a daily basis, with the 
AffecTech tools at hand but not the burden, too often overlooked by a team for whom his interests actually represent a not negligible source of revenue and future view. PLUX family is a big one to thank, doubling in size as I stepped down. To Guilherme who, before becoming the heart of dev team, customer support and pillar of interactive tutorials, established the link with NOVA for me and brought research and dissemination challenges I was pleased to contribute to. To Rui, for balancing the double-edged better than anyone. To the whole developer team, the past and future of the company, taking me from 0 understanding of these familiar phonetics to proper command. Hence, to those they let go, and to those they allowed to grow. rfreixo, gtelo, Nuno, Maria, André, Catarina, Diogo, Vanessa, Pedro... to the team behind and heart of PLUX, much needed Manuel, Joana, alongside João, who could effortlessly design a smile on anyone. To Maysa, Liliana, José... To the hands of PLUX, Paulo, Reginaldo, Tiago e à Carla, because a team is made by sharing each day, whether or not the early bird catches it first. For holding the practical knowledge and making the magic happen. To those borrowed from LIBPhys and to LIBPhys itself. Ricardo, Daniel, João, David, Cátia, Nafise, Rodrigo, Alexandra... To AICOS' loans, Ricardo... To those who suffered my early self-awareness physiology tests. To those who left an imprint in the boards I learnt to appreciate, - Filipe, Hugo. As important as the fresh air that came with Pedro, Madalena, Manuel, Patrícia, Rita, the international links (danke), the so-called miudagem and exchanges that made the place more student-ready, Katrin, Annkatrin, Takuya, Laura, le nouveau garçon... Às voltas ao quarteirão, my walks through the seven hills, olhando para o Tejo entre baloiços e escorregas, Xabregas to Madre Deus... to the soundtrack and few lines of those escapes. And then, obviously, to those who took AffecTech to heart and committed themselves to it. To those who reconnected me with heartfelt research. To Kia, for mastering the leadership of a unique, yet ahead of the curve research group. Tack! If this thesis exists that's because KTH's IxD jungle put me where I needed to be. With unbeatable partners in crime, with care, awe, respect and sincere criticism when needed, Vasiliki, Pedro, Anna, the Nottingham Mixed Reality lot, - particularly Paul and Steve - and those to be always inspired by, in my Södermalm and fika days, Jordi, Nadia, Marie Louise, Özgün, Karey, Teresa, Ylva, Madeline... letting me approach what made it into our days from the thoughts of a Bielorussian physicist with a true account of body awareness aside any buzz. Reading into design fiction, digital futures... The physicist sleeping with biomedical engineers, turned engineer sleeping with body-aware HCls... Everything is designed. Some at least dispute how. A pandemic wiped out any future plan on physiology-backed psychotherapy, deeper audio enactments, body-aware Human-Robot emotional interaction, or even the much-worked Hawaiian prom and Californian links (thanks Noura). Now inured, curtido, con un bagaje para llevar dondequiera, looking forward to contributing to the future of health technology, novel interaction design and tracking devices. 


\section{Abstract}

With the emergence of high quality low-cost personal sensors in recent years, biosignal research and the integration promises of monitoring technologies are gaining momentum. Within health and well-being domains, leveraging unprecedented machine learning processing opportunities for such technologies, wearable sensors are called upon to shake how people personally engage with health, reconnect with the body and gain self-awareness. This transformation is a change that involves the efforts of a research that is highly interdisciplinary in nature, gathering the attention of both public and private sectors. Driven by the inspiration that personal sensing entails, Human-Computer interaction and experience design teams create tools that challenge foundations, uncover the potential and limitations that pave the way to a novel, personal, form of rapport with technologies. Opening the design space, with interaction designers and engineers working hand-in-hand, enables people to look afresh at technologies that have come to stay.

This thesis is an invitation to rethink how simplistic approaches to biosignal processing enrich the potential of personal sensing in line with the fast development of what have come to be undeniably ubiquitous technologies. Moreover, the aim of taking signal processing, biomedical engineering techniques and machine learning, "Making biosensing available" to design with, is at the core of the thesis. Entering interaction design, pushing for mid tools that expose the affordances of the technologies used, embracing first-person and body-centred design and addressing how biosignal data touches sense-making, ownership and interpretation, a disruption that makes society reflect on how sociodigital materials affect our lives is sought. As a result, this thesis weaves advances in the development of biosignal processing and visualisation interfaces suitable to digital health with novel ways to interact with biosignals, addressing affective technologies, body selfawareness, and alternative biofeedback modalities that render biosignals available 
for the design of engaging interactions. On the one hand, the outcomes of this research encompass the study of a simplistic recurrent neural network paradigm suitable for biosignal and health data processing, and a closely intertwined study of biosignal data visualisation tools with the use of real-time, elaborate features for the recognition or characterisation of complex physiological phenomena. On the other hand, and more notably, the outcomes of this thesis draw on a general overview of biosignals and first-person, body-centred design techniques that lay the foundations for a major design exploration of physiology. This interdisciplinary design exploration yields insight in unpacking the relationship between people and personal affective technologies targeting self-awareness, a design stance that leverages individual experiences to unpack challenges and boundaries in the design of body-centred interactions, and the creation of bodily sensing-actuation couplings that challenge biosignal data, the input-output effect coordination needs, their interpretation, and their tangible or physical properties, by making biosignals available for interaction design. 


\section{Resumo}

Com a aparição da sensorização pessoal, de baixo custo e alta qualidade, a investigação em biosinais e as promessas de integração das tecnologias de monitorização ganharam impulso. Dentro do campo da saúde e do bem-estar, aproveitando as oportunidades sem precedente quanto ao processamento de sinais e aprendizagem automática, os sensores "wearable" são chamados a revolucionar como se participa na sua própria saúde, como reconecta com o seu corpo e se ganha maior auto-consciência. Esta transformação é uma mudança que implica os esforços de investigação que é altamente interdisciplinar, chamando a atenção do setor público, assim como o privado. Levados pela inspiração que envolvem os sensores pessoais, investigadores da área de interação homem máquina e o design de experiências criam ferramentas que desafiam os fundamentos, expõem o potencial e as limitações que prepararam o caminho para uma direção inovadora e personalizável na maneira de se relacionar com a tecnologia. Abrir o espaço do design, com designers de interações e engenheiros trabalhando conjuntamente, pode facilitar um novo olhar para umas tecnologias que chegaram para ficar.

Esta tese é um convite a repensar como certas abordagens simplificadas em processamento de biossinais enriquecem o potencial dos sensores pessoais, em linha com o desenvolvimento do que tem vindo a ser tecnologias inegavelmente ubíquas. Para além disto, o objetivo de usar o processamento de sinal, as técnicas da engenharia biomédica e a aprendizagem automática, "Tornando os biosensores disponíveis", para implementar soluções de design com os biossinais, encontra-se no centro desta tese. Entrando no design das interações, apostando por ferramentas intermédias que expõem as potencialidades das tecnologias utilizadas, abraçando o design na primeira-pessoa e centrado no corpo, e abordando como biossinais tocam a construção de significado, a sua possessão e interpretação, 
procura-se uma disrupção que faça à sociedade refletir sobre como os materiais socio-digitais afetam as nossas vidas. Como resultado, esta tese apresenta avanços nas áreas de processamento de sinais e interfaces de visualização adequadas para a saúde digital com novas formas de interactuar com biossinais, abordando as tecnologias afetivas, a autoconsciência do corpo, e as modalidades alternativas de "biofeedback" que tornam os biossinais disponíveis para o design de interações atrativas. Por um lado, os resultados desta investigação englobam o estudo de um paradigma simplista de redes neuronais recursivas adequadas para o processamento de biossinais e dados da saúde, e o estudo estreitamente ligado das ferramentas de visualização de biossinais com características avançadas para o reconhecimento ou caracterização de fenômenos fisiológicos complexos. Por outro lado, e duma maneira mais notável, os resultados desta tese beneficiam de uma abordagem geral aos biossinais e as técnicas de design na primeira-pessoa, centradas no corpo, que estabelecem os fundamentos para uma importante exploração de design sobre a fisiologia. Esta exploração de design interdisciplinar, leva a uma visão importante no decifrar a relação entre pessoas e tecnologias afetivas pessoais que procuram a autoconsciência, uma postura de design que aproveita as experiências individuais para elucidar os desafios e limitações presentes no design de interações centradas no corpo, e a criação de acoplamentos de sensorização-atuação que desafiam os biossinais, as necessidades de coordenação de efeitos entrada-saída, a sua interpretação, e as suas propriedades tangíveis ou físicas, fazendo os biossinais disponíveis para o design de interações. 


\section{Resum}

L'aparició dels sensors personals de baix cost i d'alta qualitat, han suposat un gran impuls per a la recerca en biosenyals i la integració de tecnologies de monitorització. Dins del camp de la salut i el benestar, aprofitant oportunitats sense precedents quant al processament de senyals en aprenentatge automàtic per a tals tecnologies, els sensors "wearable" estan cridats a revolucionar com la gent gestiona personalment la seva pròpia salut, com reconnecta amb el seu cos $\mathrm{i}$ guanya autoconsciència. Aquesta transformació suposa un canvi que implica els esforços d'una recerca que és altament interdisciplinària, reunint l'atenció tant del sector públic com el privat. Portats per la inspiració que comporten els sensors personals, els equips de d'interacció Persona-Ordinador i disseny d'experiències creen eines que desafien els fonaments, exposen el potencial i les limitacions que preparen el terreny cap a una innovadora i personal manera de relacionar-se amb la tecnologia. Obrir l'espai de disseny, amb dissenyadors d'interaccions i enginyers treballant conjuntament, facilita que la gent tingui una nova mirada cap a unes tecnologies que han arribat per quedar-se.

Aquesta tesi és una invitació a repensar com certs abordatges simplistes en processament de biosenyals enriqueixen el potencial dels sensors personals, en línia amb el ràpid desenvolupament del que han acabat esdevenint tecnologies innegablement ubiqües. Més enllà d'això, l'objectiu de combinar el processament de senyal, les tècniques d'enginyeria biomèdica i l'aprenentatge automàtic, "Fent els biosensors disponibles", per a dissenyar amb ells, constitueix el nucli central d'aquesta tesi. Abordant el disseny d'interaccions, apostant per eines intermèdies que exposin les potencialitats de les tecnologies utilitzades, abraçant el disseny en primera-persona i centrat en el cos, i analitzant com les dades de biosenyals tenen un impacte en la construcció de significat, la seva possessió i interpretació, es busca una disrupció que faci a la nostra societat reflexionar sobre com els materials 
sociodigitals afecten les nostres vides. Com a resultat, aquesta tesi entreteixeix avenços en processament de senyals i interfícies de visualització adequades per a la salut digital amb noves formes d'interactuar amb biosenyals, abordant les tecnologies afectives, l'autoconsciència del cos, i les modalitats alternatives de "biofeedback" que tornen els biosenyals disponibles per al disseny d'interaccions atractives. Per una banda, els resultats d'aquesta recerca engloben l'estudi d'un paradigma simplista de xarxes neuronals recursives adients per al processament de biosenyals i dades de salut, i l'estudi -estretament relacionat amb això- d'eines de visualització de dades de biosenyals amb l'ús de característiques avançades per al reconeixement o caracterització de fenòmens fisiològics complexos. Per altra banda, i de manera destacable, els resultats d'aquesta tesi es beneficien d'un abordatge general dels biosenyals i les tècniques de disseny en primerapersona i centrades en el cos, que estableixen els fonaments per a una important exploració fonamental del disseny amb fisiologia. Aquesta exploració de disseny interdisciplinària, aporta nova llum per desxifrar la relació entre les persones i les tecnologies afectives personals que busquen l'autoconsciència; una perspectiva de disseny que aprofita les experiències individuals per identificar els desafiaments i limitacions presents en el disseny d'interaccions centrades en el cos, així com també la creació d'acoblaments de sensorització-actuació que desafien les dades de biosenyals, les necessitats de coordinació d'efectes input-output, la seva interpretació, i les seves propietats tangibles o físiques, fent que els biosenyals estiguin disponibles per al disseny d'interaccions. 


\section{Resumen}

La aparición de los sensores personales de bajo coste y alta calidad, ha supuesto un gran impulso para la investigación en bioseñales y la integración de tecnologías de monitorización. Dentro del campo de la salud y el bienestar, aprovechando oportunidades sin precedentes por lo que al procesamiento de señal en aprendizaje automático respecta, para tales tecnologías, los sensores "wearable" están llamados a revolucionar cómo la gente gestiona personalmente su propia salud, cómo reconecta con su cuerpo y gana autoconciencia. Esta transformación supone un cambio que implica los esfuerzos de una investigación que es altamente interdisciplinar, reuniendo la atención tanto del sector público como el privado. Llevados por la inspiración que conllevan los sensores personales, los equipos de interacción Persona-Ordenador y el diseño de experiencias crean herramientas que desafían los fundamentos, exponen el potencial y las limitaciones que preparan el terreno hacia una innovadora y personal manera de relacionarse con la tecnología. Abrir el espacio de diseño, con diseñadores de interacciones e ingenieros trabajando conjuntamente, facilita que la gente tenga una nueva mirada hacia unas tecnologías que han llegado para quedarse.

Esta tesis es una invitación a repensar cómo ciertos abordajes simplistas en procesamiento de bioseñales enriquecen el potencial de los sensores personales,

en línea con el rápido desarrollo de lo que han acabado por ser tecnologías innegablemente ubicuas. Más allá de ello, el objetivo de combinar el procesamiento de señales, las técnicas de la ingeniería biomédica y aprendizaje automático, "para hacer que los biosensores estén disponibles" para diseñar con ellos, constituye el núcleo central de esta tesis. Abordando el diseño de interacciones, apostando por herramientas intermedias que expongan las potencialidades de las tecnologías utilizadas, abrazando el diseño en primera-persona y centrado en el cuerpo, y analizando cómo los datos de bioseñales tienen un impacto en la construcción 
de significado, su posesión e interpretación, se busca una disrupción que haga a nuestra sociedad reflexionar sobre cómo los materiales sociodigitales afectan a nuestras vidas. Como resultado, esta tesis entreteje avances en procesamiento de señales e interfaces de visualización adecuados para la salud digital con nuevas formas de interactuar con bioseñales, abordando las tecnologías afectivas, la autoconciencia del cuerpo, y las modalidades alternativas de "biofeedback" que hacen que las bioseñales estén disponibles para el diseño de interacciones atractivas. Por un lado, los resultados de esta investigación engloban el estudio de un paradigma simplista de redes neuronales recursivas adecuadas para el procesamiento de bioseñales y datos de salud, y el estudio - estrechamente relacionado con esto - de herramientas de visualización de datos de bioseñales con el uso de características avanzadas para el reconocimiento o caracterización de fenómenos fisiológicos complejos. Por otro lado, y de manera destacable, los resultados de esta tesis se benefician de un abordaje general de las bioseñales, las técnicas de diseño en primera-persona y centrado en el cuerpo, que establecen los fundamentos para una importante exploración fundamental del diseño con fisiología. Esta exploración de diseño interdisciplinar aporta nueva luz para descifrar la relación entre las personas y las tecnologías afectivas personales que buscan la autoconciencia; una perspectiva de diseño que aprovecha las experiencias individuales para identificar los desafíos y limitaciones presentes en el diseño de interacciones centradas en el cuerpo, así como la creación de acoplamientos de sensorización-actuación que desafían los datos de bioseñales, las necesidades de coordinación de efectos input-output, su interpretación, y sus propiedades tangibles o físicas, haciendo que las bioseñales estén disponibles para el diseño de interacciones. 


\section{Index}

Acknowledgements

Abstract / Resumo / Resum / Resumen iii

1 Making biosignals available: A multidisciplinary perspective 1

1.1 Project and PhD Overview . . . . . . . . . . . . . . . . 1

1.1.1 Biosignals: Taking two research perspectives . . . . . . . 2

1.1.2 AffecTech: Personal technologies for affective health . . . . 4

1.2 Thesis objectives . . . . . . . . . . . . . . . . . 8

1.3 Thesis structure $\ldots \ldots \ldots \ldots \ldots \ldots$

2 Main materials and methods 13

2.1 Main materials and methods . . . . . . . . . . . . 13

2.1.1 Multimodal biosignal acquisition platforms . . . . . . . . 14

BITalino revolution board . . . . . . . . . . . . . 15

biosignalsplux acquisition platform . . . . . . . . . . 16

2.1 .2 Biosignals . . . . . . . . . . . . . . . . . . . . . . . 17

2.1.3 Surface electromyography (sEMG) . . . . . . . . . . 19

2.1 .4 Electrocardiography $(\mathrm{ECG}) \ldots \ldots$

Photoplethysmography (PPG) . . . . . . . . . . . . . . 22

2.1.5 Electrodermal Activity (EDA) . . . . . . . . . . . 23

2.1.6 Electroencephalography (EEG) . . . . . . . . . . . . 25

2.1.7 Inertial Measurement Unit (IMU) signals . . . . . . . . . 26

2.1 .8 Breathing activity . . . . . . . . . . . . . . 28 
3 Wearable health: Simplistic processing and other advances

3.1 Making biosignals available:

Biosignal monitoring interfaces . . . . . . . . . . . . . . . 34

3.1 .1 The problem . . . . . . . . . . . . . . . . . . . 34

3.1.2 Health and Machine Learning:

The case for simplistic machine learning . . . . . . . . . . 38

3.2 Materials and methods for the biosignal developer . . . . . . . . . . . . . . . . . . 39

3.2.1 Echo state networks $(\mathrm{ESN}) \ldots \ldots \ldots$

Training the echo state network . . . . . . . . . . . . . 42

Solving an ESN system . . . . . . . . . . . . . . . . . . . 44

3.3 Echo state networks and arrhythmia. When alternative, simplistic recurrent neural topologies compete with the state-of-the-art algorithms 44

3.3.1 Holter ECGs and heartbeats of different origin . . . . . . 45

3.3.2 $\mathrm{ML}$ and intuition for wearable physiology . . . . . . . . 46

3.4 Health records, Echo State Network morphology processing capabilities and heterogeneous data . . . . . . . . . . . 52

3.4.1 Training an ESN sepsis classifier . . . . . . . . . . . . 55

3.4.2 Discussion: Predicting sepsis . . . . . . . . . . . . . . 58

3.5 Beyond classification: The path to real-time processing and new

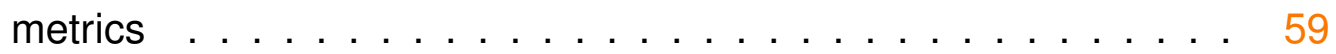

3.5.1 Monitoring physiology in real-time: HRV . . . . . . . . 60

Analysing heart rate variability $\ldots \ldots$. . . . . . . . 61

List of implemented HRV features . . . . . . . . . . . . 62

Discussion and limitations . . . . . . . . . . . . . . . 65

3.5.2 Combining biosignal features into new descriptors: The case of fatigue monitoring . . . . . . . . . . . . . . . 67

Assessing fatigue: Tests and features . . . . . . . . . 67

Combining fatigue descriptors into a global index . . . . . . 70

Discussion and limitations . . . . . . . . . . . . . . . 72 
4 Biosensing, body self-awareness and first-person design

4.1 Making biosignals available:

The body, interaction design and affective technologies . . . . . . . . . . . . . 77

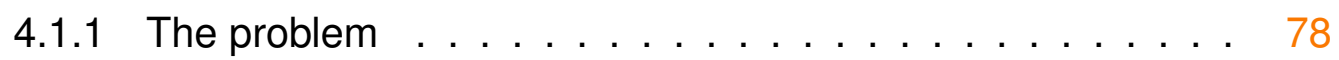

4.2 Materials and methods for the interaction designer . . . . . . . . . . . . . . . 81

4.2.1 The third wave of Human-Computer Interaction . . . . . . . 82

4.2.2 First-person perspective design . . . . . . . . . . . . . . . 84

Autobiographical design . . . . . . . . . . . . . 85

4.2 .3 Soma design . . . . . . . . . . . . . . . . . . . 85

4.2.4 Haptics and the body . . . . . . . . . . . . . . 87

4.3 Affective technology. Interacting with the body . . . . . . . 88

4.3.1 When design redefines technology . . . . . . . . . . 90

4.4 The ThermoPixels:

User-engaging affective biodata . . . . . . . . . . . . . . 91

4.4.1 Background: Affect, material approach and users' input . . 93

4.4 .2 A toolkit to design with . . . . . . . . . . . . . . 95

4.4.3 Prototypes. Affective technology design lessons . . . . . 99

4.4 .4 Limitations . . . . . . . . . . . . . . . . . . . . . 102

4.5 Designing from a first-person perspective . . . . . . . . . 102

4.5.1 Embracing discomfort . . . . . . . . . . . . . . . . 102

4.5 .2 Electrodermal haptics . . . . . . . . . . . . . . . 105

Discomfort as a design element . . . . . . . . . 106

4.6 Dare to design: Sensory misalignment . . . . . . . . . . . 106

4.6.1 First-person account insights . . . . . . . . . . . 108

Balancing on a beam . . . . . . . . . . . . 110

Suspended on a VR harness . . . . . . . . . . . . 111

4.6 .2 Limitations . . . . . . . . . . . . . . . . . . . . . . . 113

4.7 First-person Physiology: The Sensing Self . . . . . . . . . . . . 114 
Capturing body information. EMG, ECG and EDA . . . . 115

4.8 From Biodata to Somadata . . . . . . . . . . . . . . . . 122

4.8.1 Biosignals as a design material . . . . . . . . . . . . 123

4.8.2 Collaborative design of biodata interactions . . . . . . . 125

4.8.3 Biosensing-Actuation couplings . . . . . . . . . . . 126

4.8.4 Somadata and design insights . . . . . . . . . . . 128

4.8 .5 Limitations . . . . . . . . . . . . . . . . . . . . . . 129

5 Making it real. Discussion on biosignal-enhanced body interactions 131

5.1 A research outlook on biosignal-enhanced interactions . . . . . . . 131

5.2 Orchestration, design platforms and interfaces . . . . . . . . 133

5.2.1 A repertoire of biosignal effects . . . . . . . . . . . 135

5.2.2 Machine learning for biosignal interactions . . . . . . . . 137

5.2.3 Supporting the design process . . . . . . . . . . . . . . 139

A design interface candidate . . . . . . . . . . . . 141

5.3 Shape-changing breathing: supporting the design of a somaesthetic biosignal coupling . . . . . . . . . . . . . . . . . . 142

5.3.1 Deep Touch Pressure . . . . . . . . . . . . . . . . . . . . . 146

5.3.2 Breathing as input . . . . . . . . . . . . . . . . . . 148

Processing breathing data . . . . . . . . . . . . . 149

6 Conclusion and final remarks 151

6.1 General overview . . . . . . . . . . . . . . . . . . . 151

6.2 Thesis contributions . . . . . . . . . . . . . . . . . 154

6.2.1 Engineering and biosignal development contributions . . 155

6.2.2 $\mathrm{HCl}$ and Interaction design research contributions . . . . 157

Follow-up works . . . . . . . . . . . . . . . . . . 162

Concurrent works . . . . . . . . . . . . . 163

Additional publications . . . . . . . . . . . . . . 163 


\section{Index of figures}

1.1 Patient trying a VR application for chronic pain [Jones et al., 2016]

1.2 World Health Organization estimates of depression prevalence (cases) worldwide [World Health Organization, 2017] . . . . . . . 5

1.3 AffecTech expertise and research areas . . . . . . . . . 7

1.4 Thesis structure . . . . . . . . . . . . . . . . . . . 11

2.1 BITalino revolution biosignal acquisition platform [BITalino, 2013] . 16

2.2 biosignalsplux acquisition platform [PLUX S.A., 2017] . . . . . . 17

2.3 Different monitored biosignals: a) Electromyography (EMG), b) Electrocardiography (ECG), c) Electrodermal activity (EDA), d) Electroencephalography (EEG), e) Inertial measurement unit (IMU) signals and f) Respiration . . . . . . . . . . . . . . . . . .

3.1 PLUX OpenSignals software implementation of histogram (statistics) and battery monitoring assets . . . . . . . . . . 36

3.2 Study of blood volume pulse (BVP) noise in a custom-made wrist PPG sensor . . . . . . . . . . . . . . . . . . . . 37

3.3 Example of open source BITalino Hands-on coding tutorials for biosignal acquisition and processing sessions (Python Jupyter Notebooks) . . . . . . . . . . . . . . .

3.4 ESN structure, with the input layer, fixed reservoir and weighted output layer . . . . . . . . . . . . . . . . . . . . . 42 42

3.5 Example of a template sinus (normal) reference ECG heartbeat and their Supraventricular (SB') and Ventricular (VB') beat counterparts

3.6 Average healthy heartbeat minmax scaling . . . . . . . . . 50

3.7 Clinical utility rating function for septic prediction . . . . . . . . 54 
3.8 Sepsis dataset ratios of missing data (\% of not a number values, $\mathrm{NaN})$ per feature. . . . . . . . . . . . . . . . . . . . 55

3.9 ESN AUC-based input $(\gamma)$ and feedback $(\eta)$ strength parameter grid search for the classification of septic entries in ICU medical record data . . . . . . . . . . . . . . . . .

3.10 Example of an electrocardiography ECG signal and its waves and peaks [Rehua (derivative) et al., 2014] . . . . . . . . . . . .

3.11 PLUX OpenSignals software HRV real-time add-on panes, with filtering settings, selected features and generated graphs (left to right) 65

3.12 Example of a Global Fatigue Descriptor (GFD) and its dynamics for a given subject. Red (below first quartile) and green (above median) ranges denote dangerous and non-dangerous fatigue levels, respectively. . . . . . . . . . . . . . . . . . . . . 71

3.13 Support Vector Machine (SVM) fatigue classifier output for 5 subjects. Green datapoints represent non-fatigued class outputs and red datapoints represent fatigued class outputs. . . . . . . . . . . 73

4.1 2D representation of arousal-valence affect, where affect words can be placed attending to their intensity and pleasure . . . . . . . . .

4.2 Crafting arousal representation prototypes with ThermoPixels. Badge: (a) sketching, (b) layers, (c) thermal/touch exploration of liquid crystal layer, (d) connecting the nichrome wire, (e) arousal EDA sensing test, (f) wearable badge . . . . . . . . . . . . . . . . .

4.3 Crafting arousal representation prototypes with ThermoPixels. Flowers: (a) sketching, (b) arousal transitions, (c) layer structure/revealing sheets, (d) arousal EDA sensing test, (e) nichrome wire heating .

4.4 VR harness depiction, with (a) rotation axes, (b) real example flying downwards, (c) VR scaffold position . . . . . . . . . . . . 108

4.5 Body shapes to reflect on our own limitations, sketch sensor placements and decide involved body parts . . . . . . . . . . . . 114

4.6 BITalino EMG setup . . . . . . . . . . . . . . . . . . 116

4.7 BITalino ECG setup . . . . . . . . . . . . . . . . . . . . . 119

4.8 ECG signal representation . . . . . . . . . . . . . . . . . . . . 119

4.9 BITalino EDA setup . . . . . . . . . . . . . . . . . . . . 120 
4.10 EDA signal representation . . . . . . . . . . . . . . . . . . 121

4.11 Creating extra examples of first-person sensing (EEG and breathing) 121

4.12 Somadata coupling examples: Balance EMG-to-sound experience, Scarfy thermal-based EDA feedback, ACC-based sounds of synchronous movements (left to right) . . . . . . . . . . 126

5.1 Design interface for the creation of am EMG-audio feedback coupling (using PureData). . . . . . . . . . . . . . . . . . 139

5.2 Shape-changing breathing communication workflow: Processing hub on top, managing OSC communication with (shown from left to right): sensors (R-IoT), its data processing and configuration interface (jupyter and Node-RED) and the actuation effects (Arduino) 143

5.3 Node-RED UI configuration flow and browser configuration interface (bottom right) to be used during design sessions . . . . . . . . . 144

5.4 Instances of sharing a breathing biofeedback actuation via pressure inflatables on different body locations (neck, shoulder and hands) . 147 


\section{Chapter 1}

\section{Making biosignals available: A multidisciplinary perspective}

\subsection{Project and PhD Overview}

With the turn of the century, the Western society witnessed a new wave of sensing technologies that have garnered the interest of clinical psychology. With the aims of reducing the cost of accessing treatment, adding objective measurements, strengthening the client-therapist rapport or the compliance with the therapy, research has started to look at these technologies. Aligned with the rise of personal sensing, sports and physical health have capitalised the adoption of these technologies at home. With regard to mental wellbeing, though, treatments that had flourished in the online space, remote counselling, and computer-based therapy, are nowadays showing interesting paradigms using physiological sensing. Affective technologies have been unexplored, to some extent. Throughout the 2000s, research in Human-Computer Interaction ( $\mathrm{HCl}$ ), much inspired by the advent of the computation that relates or influences emotions - a novel Affective Computing field [Picard, 1997] - recognised the value of biosensing to capture affect in several experiences, or even the use of the nowadays ubiquitous smart phones to address emotion tracking through behavioural notes and metrics. This is the case of Matthews et al. [Matthews et al., 2008], one of the first instances in which phones take paper-based mood charting concepts to screens that are available 24/7, suggesting the potential of embracing technology in mental health. In the work of Lindström, Ståhl, Höök et al. on the Affective Diary [Lindström 
et al., 2006, Ståhl and Höök, 2008], biosensing is leveraged together with mobile technologies for affect self-reflection. Projects such as ICT4D yielded outcomes arguing in favour of biosensing and tracking device ecosystems aimed at the treatment of depression [van de Ven et al., 2012].

The study of interaction design surrounding affective technology usage, as seen in mental wellbeing contexts, inspires novel biosignal research that pushes the boundaries of the processing that these data undergo and how information is interpreted and handled. In this $\mathrm{PhD}$ project, biosignals are taken as the main object of study. In order to work closely with the application of biosignals in real-life scenarios, i.e. not only the processing of information but also how it is used alongside technology, what it entails and in what contexts, a focus on two research perspectives is adopted.

\subsubsection{Biosignals: Taking two research perspectives}

As of today, designing affective interactions that support emotion awareness and self-reflection is still open for research questions. Acknowledging the growth of interest towards affective technologies, new research projects set out to face a list of challenges. In all of them, biosensing and psychophysiology potentially have a major role. Biosignals need to be made available. But what does making biosignals available mean? There is room for improvement in the ways that biosignal data is being delivered and used today. The research presented in this thesis stems from two different perspectives, in other words, making biosignals available for developers and making biosignals available for interaction designers. On the one hand, from an engineering perspective, one could ask what fronts are there to extend a broader adoption of physiological monitoring. The case of wearable health is paradigmatic, as current software interfaces presenting, customising and enabling different processing mechanisms to handle biosignal features, condition the way data makes it into use cases. Richer information, fine-grained visualisation of data and uncovered features (or combinations of features) facilitate the work of developers. Biosignal acquisition platforms progress alongside learning materials and processing code snippets whose ease of code affects how technologies are adopted and refined. Furthermore, wearable health devices are crucial to probe to what extent simplistic algorithms are able to run fast, provide reliable data processing and execute without necessarily relying on a high performance 
computer. On the other hand, a range of different questions emerge if we take an interaction design perspective. How can designers leverage an information that is seldom tangible? If we consider biodata as a material, one can argue that biodata is not a malleable material to design with. Biosignals, although specific, temporally accurate or precise, face the same design challenge. This, of course, differs substantially from designing artefacts or experiences with tangible materials found in crafting practices, such as using wood, fabrics or metals. Regarding the body, designers, interaction researchers and artists have tried to capture movement, laughter, drug intake, hormonal and circadian rhythms, among other sorts of biodata in order to gain an understanding of our bodies and propose novel interpretations. A biosignal, i.e. biodata acquired by electrophysiology sensors, is a computational material, often based on fluctuations of body properties that are digitised. Research on the ways in which biosignals can be stretched, adapted, modified and interpreted is needed. How do users embrace such information and make it their own? Would that suffice to integrate psychophysiology in psychotherapy contexts? Moreover, aligned with alternative ways to relate to affective computing, the body is no longer a mere object to be measured. Paradigms that address affect as interaction rather than states to be algorithmically identified bring an interaction design alternative to the table. How can one design with biodata, i.e. data making users intimately relate to bodily properties? These properties are often addressed intangibly. However, biosignals used for - body and emotional - self-awareness, offer a fertile ground for discussion on subjective connections to biodata, feeling alive and the interpretation of data. One of the pillars of this thesis revolves around new avenues of using biosignals, inspired and approached by an interaction design research perspective. Of particular interest is the use of Somaesthetical Design [Höök, 2018], an approach that highlights the need to take the body into consideration when creating interaction experiences (introspective or not), following Richard Shusterman's Somaesthetics [Shusterman, 2012], not taking the body as a disconnected entity in the traditional brain-body dichotomy but holistically, acknowledging the role it plays in experiencing the world and in shaping our subjective interpretations.

On another front not directly covered in this thesis, there is the case of clinical psychology research. When in clinical contexts, e.g. using cognitive behavioural therapy, the construct of emotion regulation comes to relevance, inevitably making it possible to find links between physiology and self-awareness, its interpretation, 
cognitive re-appraisal, modulation of the responses triggered by an emotionally charged event, etc. Clinical psychology researchers have even fostered the

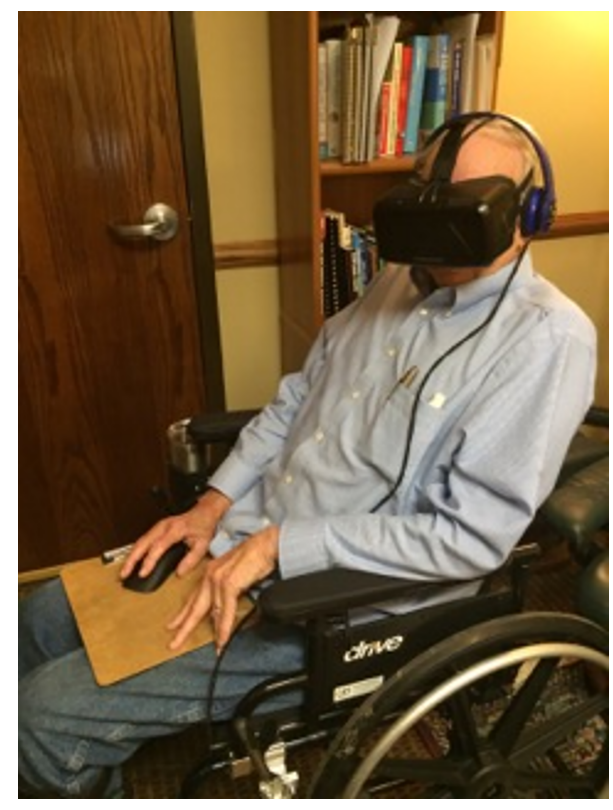

Figure 1.1: Patient trying a VR application for chronic pain [Jones et al., 2016]

use of technologies that go from virtual/augmented reality for exposure therapy addressing phobias and anxiety [Tortella-Feliu et al., 2010] - with other use cases researched in chronic pain [Jones et al., 2016] (see fig. 1.1) and post traumatic stress disorder -, the psychophysiology validation of therapy through biosensing, and for example the mechanisms for the self-assessment of moods [GarciaPalacios et al., 2013] (known as ecological momentary assessment, EMA) when out of the lab and adapting traditional paper-based questionnaires into simplified versions on portable screens (mobile phones or wearables).

\subsubsection{AffecTech: Personal technologies for affective health}

The interest in the interplay of mental wellbeing, self-awareness and sensing technology has crystallised through several public-funded international and interdisciplinary projects that the European Commission has put forth. The AffecTech $\mathrm{H} 2020$ project, bringing together private and public stakeholders, is unique in achieving the direct involvement of original equipment manufacturers. But the 
public health challenges of mental wellbeing are vast. This thesis has benefited from the H2020 support and falls under the overarching AffecTech goals.

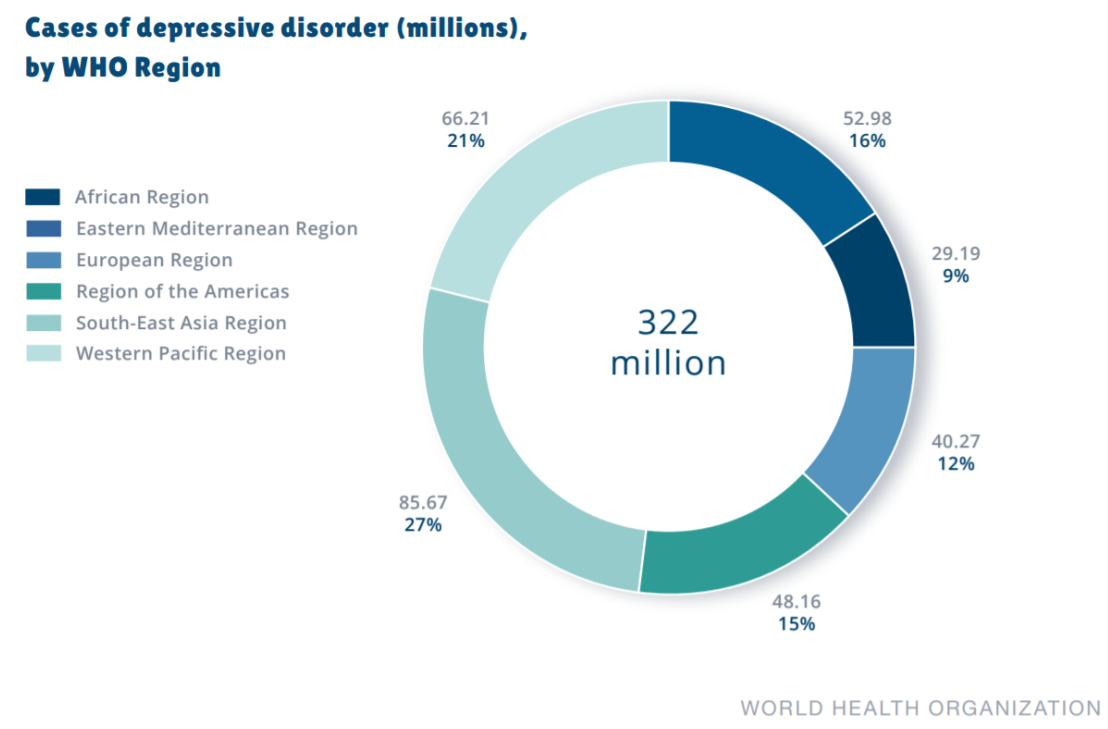

Figure 1.2: World Health Organization estimates of depression prevalence (cases) worldwide [World Health Organization, 2017]

According to the World Health Organization [World Health Organization, 2017], depression, for instance, is estimated to have a prevalence of 322 million cases worldwide as of 2017 (see fig. 1.2). Other problems like suicide, or affective disorders, such as Anxiety and Bipolar / Manic Depressive disorder are not of lesser concern. AffecTech efforts are timely, as European Union's efforts on creating international research networks addressing mental wellbeing acknowledges the utmost priority that Europe puts in a global problem causing staggering economic and health consequences. This can be seen throughout different research networks around the field but focusing in a wide range of particular aspects such as policy development, technology transfer, psychoeducation and awareness. Examples of such networks are [Ubicom Mental Health, 2015, TEAM ITN, 2016, eMEN, 2019]. AffecTech, in particular, highlights the personal aspect of the affective technologies, i.e., how biosensing, interactions and, potentially, clinical interventions, can better adapt to the user, be customised or respond to the different challenges that different health conditions and individual subject needs pose.

The AffecTech Innovative European Training Network is an ambitious consor- 
tium which is conformed by 11 institutions (8 Universities) throughout Europe, creating an unprecedented interdisciplinary framework to target Mental Health and technology goals:

- Lancaster University - ULANC; Lancaster, UK

- Trinity College Dublin - TCD; Dublin, Ireland

- University of Oxford - UOXF; Oxford, UK

- Università di Pisa - UP; Pisa, Italy

- Universitat Jaume I - UJI; Castelló, Spain

- Università Cattolica del Sacro Cuore - UNICATT; Milano, Italy

- Boğaziçi University - BU; Istanbul, Turkey

- Philips Electronics B.V. - PHILIPS; Eindhoven, The Netherlands

- Leeds teaching Hospitals National Health Service Trust - NHS; Leeds, UK

- PLUX Wireless Biosignals S.A. - PLUX; Lisboa, Portugal
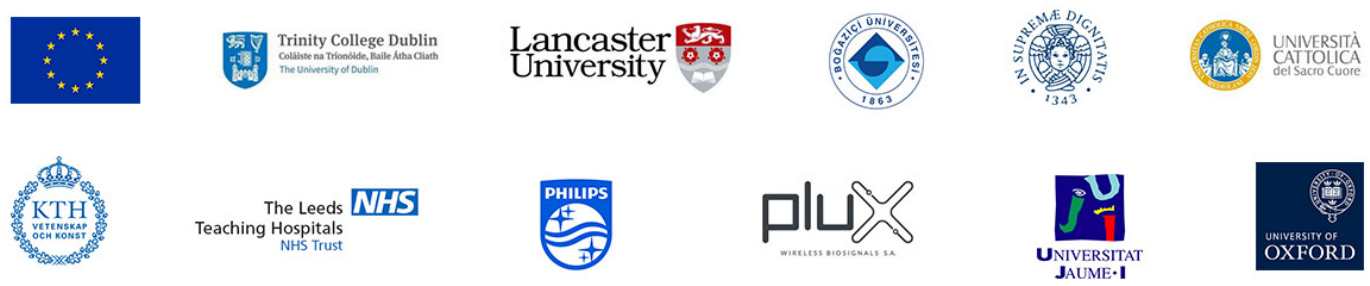

AffecTech gathers outstanding yet fragmented expertise in developing personal systems for mental health. AffecTech expertise areas are Electrical Engineering / Biosensing, Clinical Psychology and Human-Computer Interaction:

The contribution of clinical psychologists to the creation of affective technologies is of relevance. No other professionals are better equipped to assess which interactions make sense to a potential psychotherapy intervention. With background on adapting traditional therapy to the digital domain (e.g. eHealth, remote therapy, virtual reality, supported by personal sensing, etc.) the health professionals are 


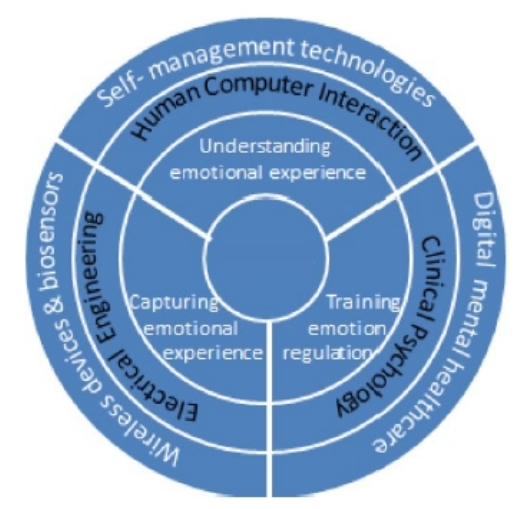

Figure 1.3: AffecTech expertise and research areas

in charge of the task of delineating use case needs, identify the most researched commercial platforms and capture the latest advances adopted clinically. Electrical Engineering / Biosensing must facilitate access to low-cost, high performance and multimodular biosensors that can be integrated in wearables and/or affective health interactions. By multimodularity, we refer to the possibility of integrating different acquisition sources in the same platform or interaction, as requested by the specific needs of the designed experience, in other words allowing the inclusion of heart data, muscle data, skin conductance, or breathing among others. These technologies must ideally operate through several programming interfaces, making it simpler for designers to explore different features, representations and processing algorithms that are the object of study of engineers. Human-Computer interaction must leverage the expertise on the creation of experiences related to affective health, provide the tools to review the state-of-the-art and adopt novel ways to address technology-mediated interactions that touch upon mental wellbeing, emotions and self-reflection.

The idea behind an interdisciplinary research project like this, is that researchers from the different areas are able to pave the way to explore intersections of the fields, understand the needs and limitations of all the areas involved and receive training that facilitates approaching the technology to the clinical practice, the therapist goals to the engineers and the expertise in designing interactions deploying the technology smoothly to increase acceptance, provide meaning, actively engage the subjects involved and ensure integrity, privacy and respect for the user. To pursue the necessary dialogue across research areas, a complete year 
of the project is devoted to a series of 6 training events that focus on one of the key disciplines for a week, involving academics of reference and unpacking use cases from the commercial sector. These complement research internships scheduled to take place during the project, so that the researchers are exposed to first-hand knowledge and development from areas other than their own background.

With the framework of the mental wellbeing domain, the UJI-PLUX industrial $\mathrm{PhD}$ project addresses the integration of Human-Computer interaction and biosensing expertise drawing on contributions by all the network participants. The $\mathrm{PhD}$ project is organised in order to understand the technical and practical knowledge of the state-of-the-art and research methodologies of AffecTech with the aim of developing joint experiences integrating wearable systems and interactive applications for capturing, understanding and reflecting upon affective health. This approach should leverage the use of wearable sensors, capturing meaningful events through physiological components of emotions, and put a strong emphasis on the creation of interactions that feel meaningful for the user that embraces affective technology to better understand and reflect on her/his mental wellbeing.

\subsection{Thesis objectives}

The work presented in this thesis is eminently interdisciplinary, between engineering or developer roles and that of an interaction designer, seeking to use technology to spur reflection on the body. Thanks to the AffecTech [AffecTech, 2017] and industrial scope of the PhD, this thesis is faced with the Making biosignals available challenges (see the framing of the problem in section 3.1 and 4.1) that would have a direct impact in researcher peers. In other words, the biosignal stakeholders are not end users of an interaction only, but the developers behind biosignal acquisition and visualisation platforms and the interaction design researchers that seek the inclusion of materials that unpack body/physiological information otherwise not achievable. A suitable approach to map this research space, would be that of investigating what of biosignal users. However, this thesis opts for an alternative. Two disjoint perspectives (engineer/developer and interaction designer) are taken. Following these, a list of unresolved topics is devised. To begin with, being immersed in a biosignal platform development environment, the limitations or lack of material in accompanying the novice biosignal user is experienced first-hand. Contributing to the development of such platforms is a means to 
expose novelty fronts. A development team is faced with the question on how to best support the use of biosignal visualisation software. Moreover, in most of the use case scenarios that reach to the development team from psychophysiology studies, real-time assessment - not only acquisition - appears consistently. This, of course, goes in line with other wearable developments that have seen the light in recent years, i.e., health risk alarms and recognition of patterns, all of them with the capability of being delivered in real time. But, real-time processing requires elaborate algorithms that leverage computations that are fast and accurate. As a consequence, Making biosignals available is translated in the following objectives:

- Development: Explore the ways to extend the handling of biosignals, such as tools for those working at the forefront of the development of processing stages, dealing with wearable health, algorithms and realtime interfaces that expose characteristics of the physiology.

01. Characterise the main elements present in the acquisition of biosignal data and their processing, i.e. characterise the most frequently used biosignals in physiology and affective computing alongside the acquisition hardware and data visualisation interfaces.

O2. Commit to the development of support material that accompanies the discovery and usage of biosignals, exposing specific features, enabling end user actions and transferring the control of the platform to them.

O3. Study computationally undemanding machine learning $(\mathbf{M L})$ tools. Investigate classification paradigms that are potentially suited to wearable biosignal devices with limited computing capabilities, but able to be customised by the user and integrated with biosignal data acquisition software.

O4. Study the possibility to customise and integrate several features to capture complex characteristics from physiology data .

O5. Study the transition of algorithms for biosignal processing into real-time physiology platforms.

Furthermore, assuming a fast processing and recognition of (real-time) specific biosignal features is feasible, this thesis is faced with the open question on what to do with such features. Biosignal devices are capable of capturing body properties 
to be exposed, used and reflected upon. This research is committed to create an introductory description of biosignals and the accessible low-cost physiology acquisition platforms to be used. Once this is set, it is possible to step into the study of how biosignals and their features are handled. The aim consists facilitating the work of both (a) the developer who wants to delve into using biosignals to study, create experiences, or utilise the physiology of the body, and (b) the interaction designer, invited to eagerly embrace a body technology that can be adapted, understood, defied and used at anyone's convenience. With the core topic of affective technologies and bodily self-awareness backing up the thesis, a list of objectives with respect to Interaction design research is prepared:

- Design: Study ways to alter, interpret and process biosignals, with an interactive design perspective focusing at body/emotion self-awareness.

O6. Establish a design stance, novel or literature-based, that helps the researcher address the interaction of emotion, body physiology and interaction boundaries.

O7. Through material explorations and crafting practices that combine technology and physical materials, approach the interpretation of biosignals that connect to either body or emotional aspects of the self, its representations and meanings from a personal view.

O8. In line with $\mathrm{HCl}$ trends on explicitly exposing materials so that they can be worked, adapted and changed, approach alternative ways to make biosignals available as a design material. The goal is that biosignals are ultimately presented in the form of tangible representations or artefacts that connect to the body, so that one can engage vividly with them.

O9. Through $\mathrm{HCl}$-Biosensing exchanges, address how design platforms support the creation of new ways to interpret biosignals, own them, approach them easily, and adapt them in a way that relates to our bodies. 


\subsection{Thesis structure}

This thesis is organised in the following structure: The $\mathrm{PhD}$ scope and project framework is presented in chapter 1, where the goals and two perspectives followed are introduced. A description of the main materials and methods utilised follows. The thesis research is divided in Part I and Part II (see fig. 1.4), in accordance to the perspectives presented in section 1.1.1. Both parts contain the description of specific materials and methods that accompany the research questions and the resulting insights from the studies conducted.

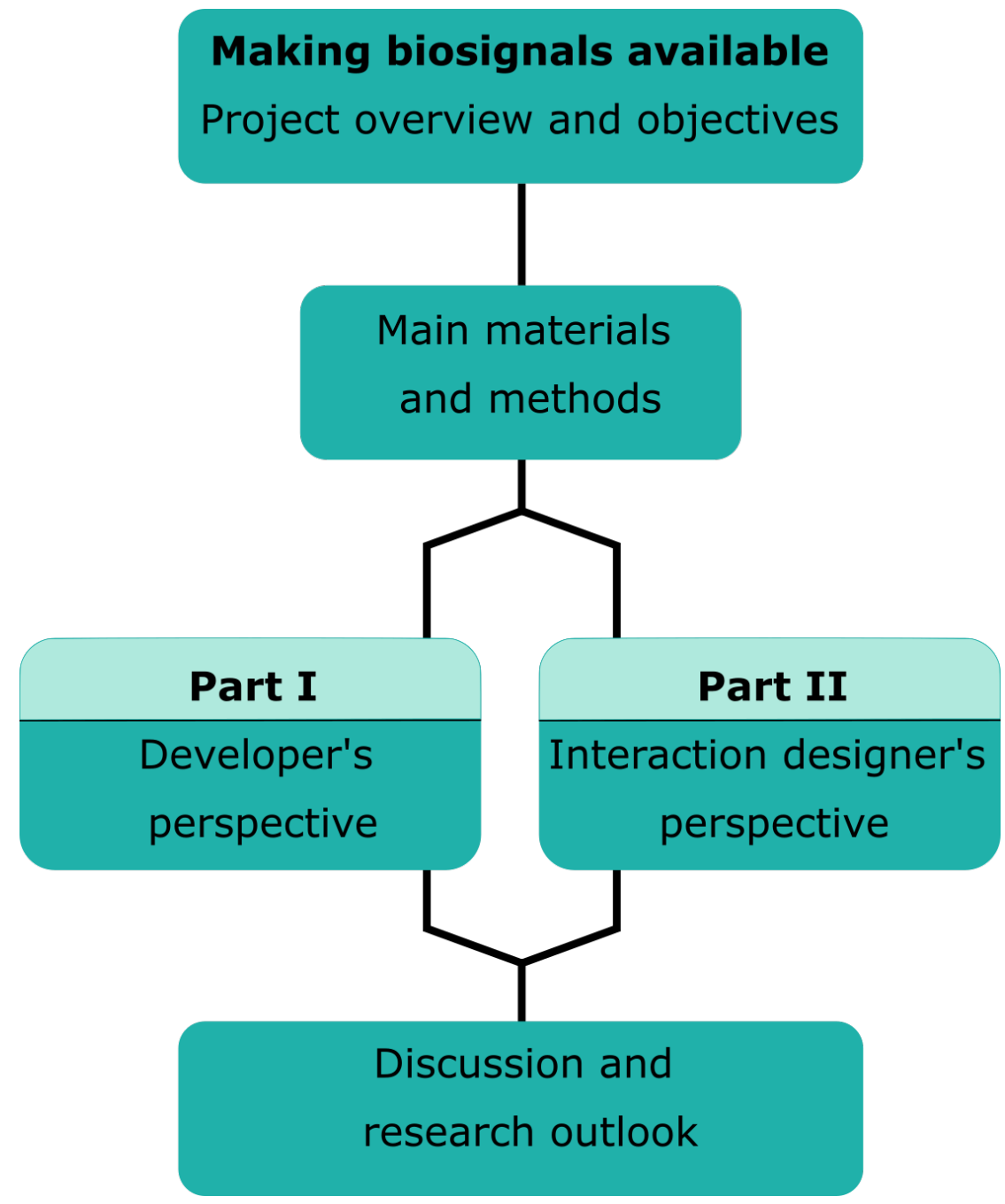

Figure 1.4: Thesis structure 
As opposed to common practice, this thesis does not present a state-of-the-art section at the beginning. Instead, the state of the art of the different perspectives addressed, is unpacked and distributed throughout the multiple studies presented in their respective sections. Part I contains the Making biosignals available research from the engineering or developer perspective (chapter 3 ), delving into wearable health use cases, addressing current progress and issues, and presenting results that argue in favour of progress done in biosignal interfaces, advances in simplistic yet efficient approaches for signal processing, and the interest in real-time processing and novel feature combinations. Part II (chapter 4) contains the Making biosignals available research from the interaction design perspective, focusing on design explorations conducted in interdisciplinary teams, addressing state-of-the-art approaches and issues, presenting studies with users/designers and going deeper into $\mathrm{HCl}$ and outcome reflections about biodata as a design material of relevance to affective and self-awareness technology. The thesis ends with a chapter (chapter 5) providing a general reflection on how to make biosignalenhanced interactions real. A discussion on future developments of interest with respect to making biosignals available is given, together with an example of interaction design leveraging both $\mathrm{HCl}$ and engineering takeaways and a look into research avenues that can continue to foster the use of biosignals in interaction design research, affective technologies and $\mathrm{HCl}$, giving way to conclusions and final remarks on aspects in which this thesis falls short or where there still room for further research. 


\section{Chapter 2}

\section{Main materials and methods}

\subsection{Main materials and methods}

The study of biosignals and how they can be made available for developers and interaction designers required an understanding of such representations and access to highly reliable and easy-to-use low-cost platforms that acquire them. In this section we present materials that have been at the core of all the studies that make up this thesis:

- Biosignals

- Biosignal acquisition platforms

Biosignals are time representations of changes in energy produced in the body. These changes correspond to energy variations of different nature, such as electrical, chemical, mechanical, and thermal (as presented in table 2.1). Examples of the measurement of mechanical changes are those of position, strain or rotations found for instance at muscle level or heart. Electrical changes can be measured as voltage signals - also measured by means of charge or conductance -, at the base of electrophysiology. Examples of these signals are electromyography (EMG), electrocardiography (ECG), electroencephalography (EEG), electrodermal activity (EDA) and electrooculography (EOG), covering the measurement of muscle, heart, brain, sympathetic nervous system activation and eye movement activity, respectively (see also section 2.1.2). Thermal changes focus on fluctuations of temperature, such as those measured on the body surface. 
Chemical changes take the form of concentrations or exchanged energy estimates, as found in the measurement of $\mathrm{pH}$ characteristics, metabolites present in body fluids or other substance or hormonal concentrations.

With the turn of the $21^{\text {st }}$ century and the advent of the digital era, the advances in the field of electronic components that spurred the development of computing, instrumentation, and algorithms left their impact on medical and biosignal devices. Biosensing and electrophysiology technologies were greatly improved, ready for the study of body functions and health monitoring in the context of clinical research. As technologies grew, the miniaturisation and reduction of costs contributed to the growth of biosensing monitoring technologies beyond clinical settings as well.

Table 2.1: Parameters and type of energy measured through body sensing. Adapted from [Semmlow and Griffel, 2014].

\begin{tabular}{l|l|l} 
Energy & Changing parameter & Measurement examples \\
\hline Mechanical & Position, force, torque, pressure & $\begin{array}{l}\text { Muscle contractions, cardiac pressure, } \\
\text { muscle movement }\end{array}$ \\
\hline Electrical & Voltage, charge, current & EMG, ECG, EEG, EDA, EOG \\
\hline Thermal & Temperature & Surface body temperature \\
\hline Chemical & Concentrations, exchanged energy & $\mathrm{pH}$, oxygen, hormonal concentrations
\end{tabular}

A direct consequence of such rapid expansion is the creation of the sports \& health monitoring markets that fill up the mobile app stores and provide remarkable revenues in the ubiquitous computing paradigm that we live in. The democratisation of the study of biosignals, however, comes with interesting possibilities such as a better understanding of the self and a richer, unprecedented way to interact with technologies that accompany us. This yields an opportunity to define alternative ways to live an affectively healthy life.

\subsubsection{Multimodal biosignal acquisition platforms}

As the maturity of open access physiology databases [Goldberger et al., 2000] backs up the improvement of processing algorithms, low-cost hardware platforms help populate the open source space [da Silva, 2018] where users embrace biosensing, share ideas and drive the future of biosignals applied in different areas. Furthermore, the biosignals that were once limited to hospitals and clinics, or in specialised research labs, addressed in classical texts of physiology, are nowadays 
accessible in virtually any context by means of wearable technologies. In the review of Heikenfeld et al.[Heikenfeld et al., 2018], an interesting account of the transition from lab tracking to wearables during the 20th century is offered along an in-depth overview of body sensing mechanisms not only restricted to electrophysiology. The field of affective computing has consistently found in biosignals a relevant source of information [Giannakakis et al., 2019]. Besides, the fact that biosensing platforms have jumped off the clinic has contributed to embracing them alongside other technologies like movement tracking, traditionally linked to behavioural and psychophysiology labs.

The research presented in this thesis is concerned with studies utilising BITalino and biosignalsplux [PLUX S.A., 2017]. Exceptionally, a particular case of using the Grove GSR Arduino accessory [Grove - Seeed Studio, 2014] for galvanic skin response sensing is found in section 4.4. With objectives that range from out-ofthe-lab psychophysiology tracking [Can et al., 2019, Can et al., 2020a, Can et al., 2020b] to new perspectives in interaction design [Umair et al., 2018, Umair et al., 2019b, Alfaras et al., 2020b] recent affective technology studies of interest have often addressed biosignals through other available biosignal research platforms such as Empatica E4 [EMPATICA, 2020], or even commercial wearables such as the Samsung Gear S2 [Samsung, 2015], among others.

\section{BITalino revolution board}

BITalino [BITalino, 2013] is a biosignal acquisition board implementing an allin-one hardware design, with a variety of pre-connected biosensing blocks. The board presents up to 6 input channels, 2 output channels for buzzer actuation or LED light, a pulse wave modulator port, a Bluetooth (or Bluetooth Low energy) communication module, a microcontroller unit and a power module that connects to the Li-Po battery supplying 3.7V for operation and charging. In its revamped revolution version, ready-to-use sensors to track muscle, heart, electrodermal, motion and brain activity coexist in a single board. The board can be found in three different kit modalities: the Board kit, the Plugged kit, where sensors are interchangeably connected by cables and the Freestyle kit, where all components, from communication, computing core, power supply to sensing modules are disconnected for prototyping purposes. In the Board kit, electrophysiology sensors are presented in the form of preattached modules. However, its modular 
board design allows to break up specific sensing modules to free channels and solder multiple sensors that better suit the research purposes of the developers. Capable of acquiring biosignals at $1000 \mathrm{~Hz}$ sampling rates, BITalino can stream data continuously for more than 12 hours.

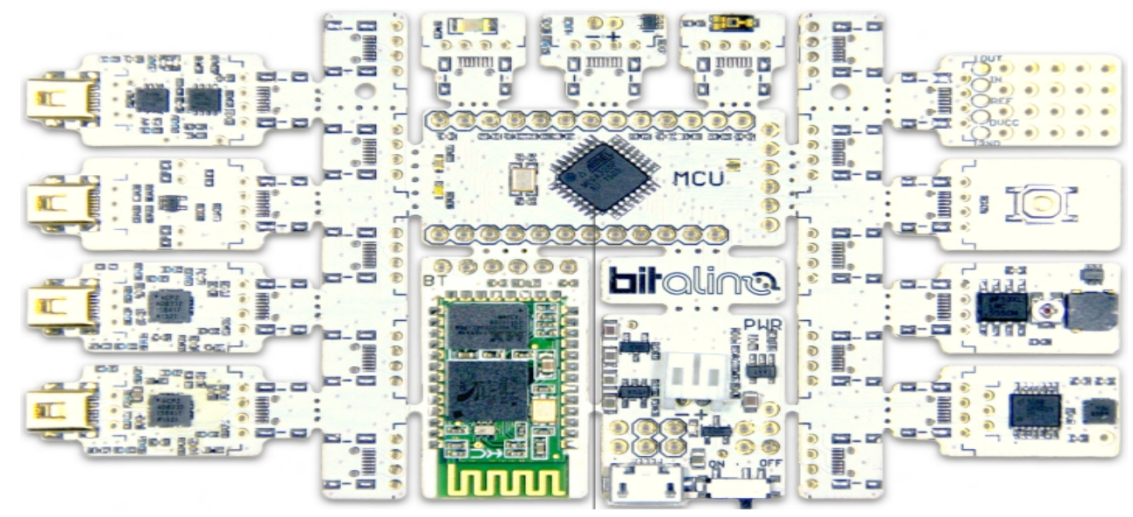

Figure 2.1: BITalino revolution biosignal acquisition platform [BITalino, 2013]

By means of specific requests from a wide range of application programming interface (API) options, data from all the channels is streamed wirelessly to a device which pairs and issues the data requests, be it a computer or a smartphone. Its sensing variety makes BITalino perfect for biosignal exploration and lab activities. The kit includes all the basic accessories needed to get started in physiology computing, with a relevant tradeoff between low cost and reliable measurements. The analogue signals are digitised at a 10 bit resolution. Along with a cross-platform and freely available software, BITalino enables instant biosignal data visualisation and recording out-of-the-box. Moreover, extra sensors such as breathing activity, blood volume pulse and others can complement the kit.

\section{biosignalsplux acquisition platform}

biosignalsplux [PLUX S.A., 2017] is a high resolution acquisition platform presented in the form of a 4-channel or 8-channel hub that enables the measurement of multimodal biosignals concurrently. With a closed and simple form factor, the platform is aimed at advanced research studies employing physiology signals. Thanks to its vast sensor choice, from common heart, electrodermal, brain, motion and muscle activity to specialised sensors such as blood oximetry, angle tracking, blood volume pulse and temperature, among others, biosignalsplux offers many 
possibilities for personal sensing research uses. The hub is capable of handling several channel acquisitions at very high sampling rates $(3000 \mathrm{~Hz})$ for human physiology. Data is captured at a $16 \mathrm{bit}$ high resolution and streamed wirelessly through Bluetooth, for a total duration that can reach 10 hours. Besides, the hub incorporates a digital port for communication and synchronisation purposes, and allows internal memory storage.
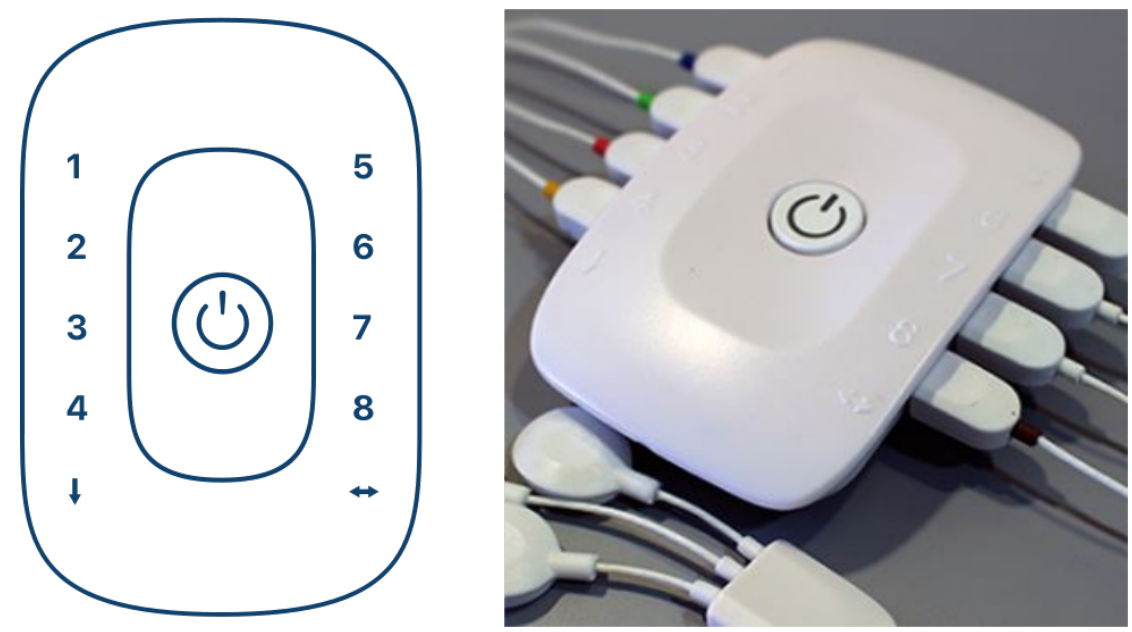

Figure 2.2: biosignalsplux acquisition platform [PLUX S.A., 2017]

Its multi-channel approach has led to a broad platform adoption in the context of physiotherapy, due to the interest of tracking several muscles simultaneously. However, the many sensing modalities has paved the way for physiology research in other domains, such as high performance sports, cognitive fatigue and ergonomics.

\subsubsection{Biosignals}

The research focus of this thesis is set on a subset of biosignals present in the BITalino revolution do-it-yourself (DIY) low-cost biosensing platform [BITalino, 2013, da Silva et al., 2014, Batista et al., 2019] that backed and inspired the research in affective technologies. These, although not an exhaustive list, are to some extent physiological signals that have become standard for physiology monitoring research - slowly crossing disciplines and making their way into affective health tracking, interaction design, and other domains of interest.

This section presents a selection of biosignals that can be incorporated into the creation of new technologies for affective health. 


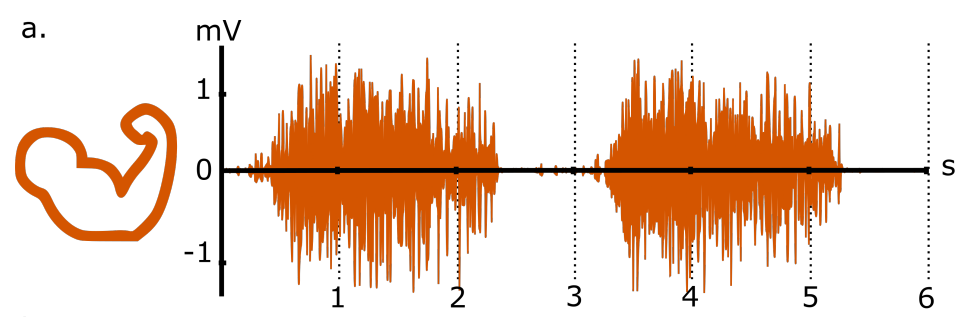

b.

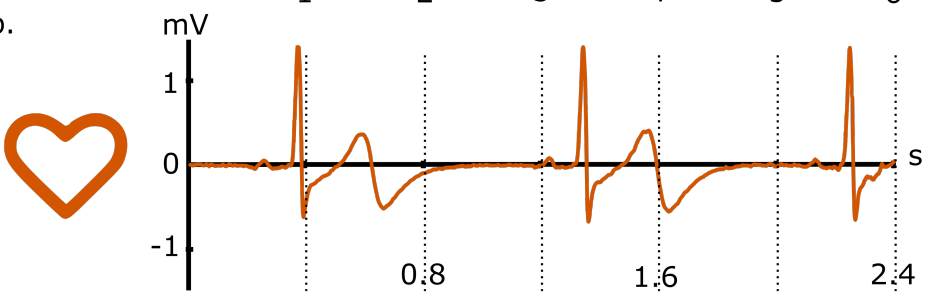

c.

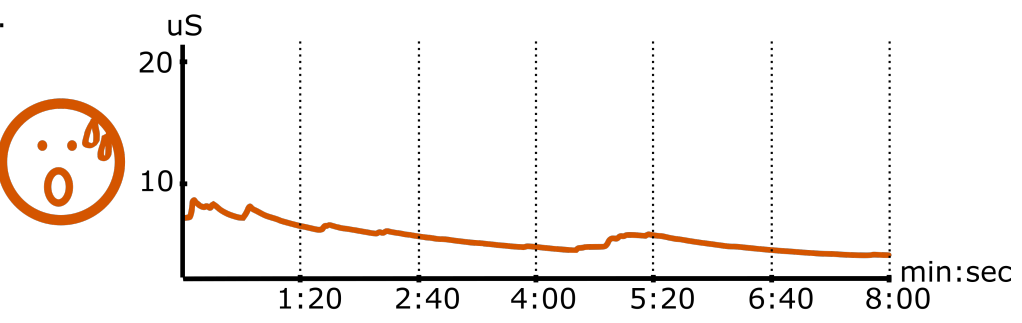

d.

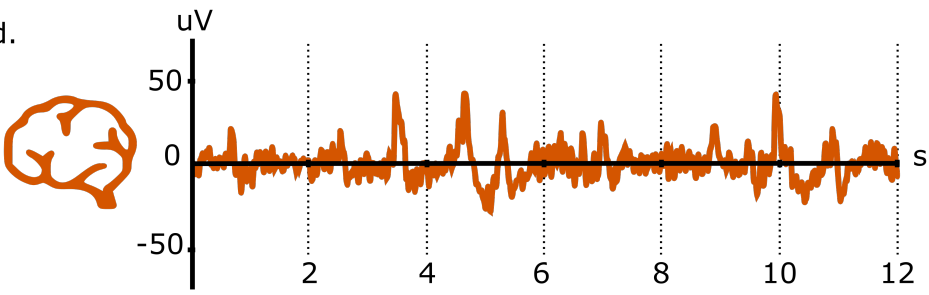

e.

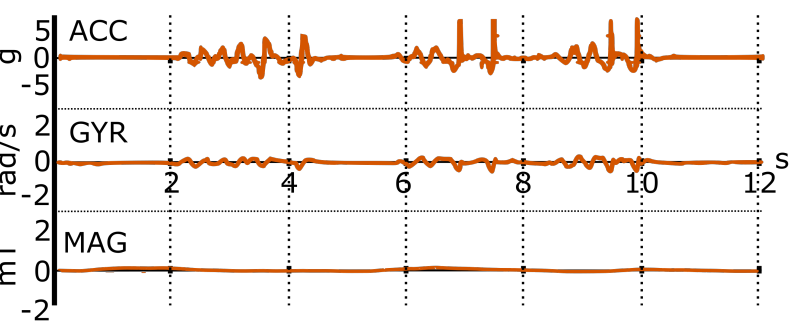

:

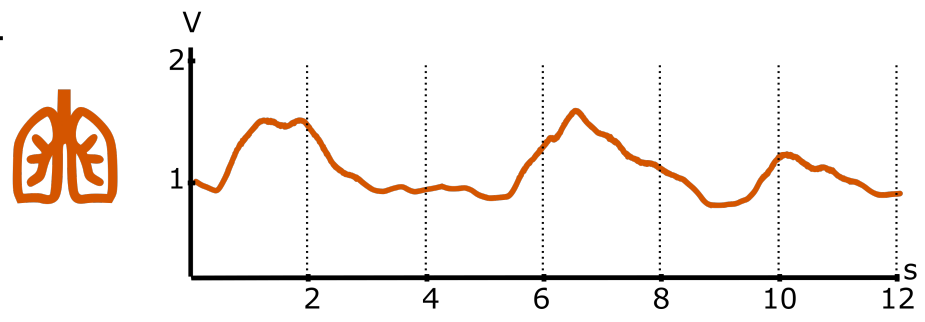

Figure 2.3: Different monitored biosignals: a) Electromyography (EMG), b) Electrocardiography (ECG), c) Electrodermal activity (EDA), d) Electroencephalography (EEG), e) Inertial measurement unit (IMU) signals and f) Respiration 
This is a collection of relevant biosignals for psychophysiology research. For each of them, this section offers a brief, systematic description on 1) How it works, summarising the basic physiological principles that provide the biosignals energy observables; 2) What can be extracted from the collected biosignal; 3) Where the biosignal can typically be collected in the human body; 4) When, or how often, the signal should be sampled describing the concerns on the timing of the acquisition and in particular the typical sampling frequency of each biosignal; and 5) Limitations of the biosignal acquisition and processing with the challenges of noise or signal artefact. All of them are examples of signals that we have addressed in our research. This non-exhaustive selection offers a good starting point for researchers interested to integrate biosignals in their design of technologies for wellbeing and mental health.

\subsubsection{Surface electromyography (sEMG)}

How it works: The recording of the electrical activity produced by skeletal muscles receives the name of electromyography (EMG). Human muscles are made up of groups of muscle units that, when stimulated electrically by a neural signal, produce a contraction. The recording of the electrical activity of the muscles (voltage along time), traditionally relying on intrusive needle electrodes (intramuscular), is easily accessible nowadays by means of surface electrodes that capture the potentials of the fibres they lay upon. The result of this measurement is a complex surface electromyography signal (sEMG) that reveals data about movement and biomechanics of the contracted muscles (see figure 2.3a).

What: Electromyography signals inform about the contraction of specific muscles and parts of the body. The EMG signal consists in the time representation of rapid voltage oscillations. Its amplitude range is approximately $5 \mathrm{mV}$. In terms of signal analysis, the EMG allows the assessment of several aspects such as muscle contraction duration, the specific timing at which movements or contractions are activated, the presence of muscular tension or fatigue, and the extent to which different fibres (area) are contracted. The analysis is conducted through noise filtering, together with feature extraction that yields contraction onset detection, the estimation of signal envelopes, and the computation of average frequencies. This lets subjects deepen their understanding of movement strategies, very relevant for embodied art and sports performance, improve muscle coordination, or even 
reveal existing movement patterns that they are unaware of.

Features: Onset instants; Max amplitude; Instant of maximum amplitude; Activation energy; Envelope;

Where: Having become the standard in EMG monitoring, bipolar surface electrodes consist of three electrodes. Two of them (+/-) must be placed close to each other, on the skin that lies on top of the muscle under study, along the fibres' direction, while the third one is placed in a bony area where no muscular activity is present. This allows the measurement of electrical potential differences with respect to a common reference, yielding a unique signal that represents the muscular activity of the area.

When/Frequency: Given the fast muscle-neural activation nature of EMG signals and the presence of different active muscles contributing to the same signal, muscle activity must be acquired at sampling rates no lower than $200 \mathrm{~Hz}$ frequencies. Working at $500 \mathrm{~Hz}$ is desirable, while a sampling rate of $1000 \mathrm{~Hz}$ guarantees the tracking of all the relevant events at a muscular level.

Limitations: Surface EMGs are intrinsically limited to the access to superficial muscles. This is compromised by the depth of the subcutaneous tissue at the site of the recording which depends on the body composition of the subject, and cannot unequivocally discriminate between the discharges of adjacent muscles. Proper grounding (reference electrode attached to a bony inactive muscular region) is paramount to obtain reliable measurements. Motion artefacts and muscular crosstalk compromise the assessment of the muscle activity under study. In this context, interference from cardiovascular activity is not uncommon, particularly in areas such as chest and abdomen. The presence of power supplies and mains (power line) in the vicinity poses the risk of $50 \mathrm{~Hz}-60 \mathrm{~Hz}$ interference.

\subsubsection{Electrocardiography (ECG)}

How it works: Electrocardiography (ECG) is the measurement of the electrical activity of the heart. The ECG consists of the tracking of the cardiac muscle contraction that results from the electric depolarisation-repolarisation cardiac cycle. In every cycle, the heart undergoes changes in the distribution of electrical charges along with the muscular tissue, which appear in ECGs as a particular wave variation in electrical potentials along time (see figure 2.3b).

What: Electrocardiography signals inform about the activity of the heart, cycle 
after cycle. The electrical signal, which presents a particular shape of well-studied peaks and troughs, reveals a pattern that informs about the heart functionality. In terms of signal analysis, ECGs and the annotation of the so-called fiducial points, allow the segmentation of the signal that leads to the extraction of features like amplitudes, peak to peak time intervals, and associated heart rate. Moreover, the analysis of the shape of the specific waves represented in the signal is linked to the correct functioning of the particular parts of the heart, which is crucial in order to spot the origin of problems. ECG analysis in time, basically built upon the peak to peak assessment and heart rate, allows the understanding of phenomena like arrhythmia, the response to physical exertion (sports), or even the presence of stressors such as cognitive demanding tasks or emotional stimuli, addressed often by means of heart rate variability (HRV).

Features: Beat instant (R wave); Heart rate; Inter-beat interval standard deviation; Heart Rate Variability; Detected waves (PQRST); Signal to noise ratio;

Where: ECG measurements are based on what is known as a lead, i.e. the assessment of electrical potential differences between two locations with a common reference point. A different lead corresponds to the measurement of heart activity from a different angle, i.e. presenting complementary signal shapes that contribute to a deeper analysis of the generated signals. Leads have been standardised through the practice of cardiology since ECG was developed. With ECG being part of all medical routine checkups, the standard 12 multi-lead (electric potential measured from different angles) setting continues to be common in clinical settings. The mechanism underlying the acquisition of an ECG signal lead consists of the placement of two electrodes (+/-) in a specific location. These track the electric activity with respect to a common reference point on the body. Both signals are subsequently subtracted, providing the desired signal representation. Limb, augmented limb, precordial, and chest leads, with clear symmetrical electrode placement guidelines on arms, legs or chest continue to be highly used in ECG studies. However, research in single lead settings and exploration of alternative electrode placement such as hands and neck have been gaining attention in recent years thanks to the pursuit of simplified assessment techniques in line with ubiquitous computing possibilities out of the lab.

When/Frequency: Heart activity during a cycle comprises the electrical activation of different regions of the heart. For this reason, the use of relatively high sampling rate frequencies is strongly recommended. While technologies working at frequen- 
cies below $100 \mathrm{~Hz}$ can extract peak to peak interval features in the time domain, insufficiently high sampling rates might miss capturing fast dynamics relevant for the assessment of the heart function important in specific contexts and cardiovascular conditions. Hence, a desirable working regime of $250 \mathrm{~Hz}$ is recommended to capture a reliable ECG shape, with clinical guidelines even pointing at $500 \mathrm{~Hz}$ or more.

Limitations: Electrocardiography, which is based on the measurement of heart electrical potential through direct contact on the skin, suffer from the presence of motion artefacts and EMG-related interference. While ECG has experienced great advances in the development of comfortable electrodes, challenges remain in terms of making the measurement less intrusive given the needed skin contact. Whereas the effects that physical and emotional activity have in ECG constitute an opportunity to focus on the subject's health at different levels, an ECG study must ensure access to mechanisms or guidelines that help having the sources of ECG variations under control - this could be the case of the task forces or governmental-sponsored committees created to provide lists of ECG-related physiological parameters that help rule out misleading information, as well as the use of multi-modular biosignal tracking, other sensors, cameras or behavioural monitors helping to tell apart ECG phenomena from movement and other artefacts.

\section{Photoplethysmography (PPG)}

A wearable ECG alternative to heart activity monitoring that has gained relevance in recent years is that of blood volume pulse (BVP) monitoring, also known as photoplethysmography (PPG). The obtained signal has different dynamics from those of ECG signals, does not provide accurate systolic-diastolic cycle information, but enables low-cost electrode-free heart rate activity tracking.

How it works: Variations in the amount of blood or air inside organs can change the volume within those organs. Measuring these volume changes is a mechanism called plethysmography. A plethysmogram obtained optically is called photoplethysmogram. Photoplethysmograms are used to detect changes in blood volume inside the microvascular surface area of a tissue [Shelley et al., 2001]. Photoplethysmography is a straightforward, non-invasive, portable, and inexpensive technique that uses light-emitting and light sensor diodes to detect volumetric changes of the blood by measuring variations in the absorption of a light shined on 
(or through) the skin. PPG uses include assessing the autonomic nervous system (ANS) function, and the signal is trackable under a wide variety of off-the-shelf personal and medical-grade measurement devices. Furthermore, wearable and unobtrusive PPG-based monitoring systems provide early symptom detection and diagnosis of physical and psychological health conditions in a consistent and realtime manner [Castaneda et al., 2018]. This mechanism has gained momentum as it has been a key tracking feature of widely commercialised smartwatch wearables. One of the drawbacks of the sensor when offered in these form factors, though, is the operation at low sampling rates that are usually not user-settable, hence presenting a significant trade-off between the quality or simplicity of the information to be extracted and the autonomy of a comfortable wearable.

\subsubsection{Electrodermal Activity (EDA)}

How it works: Electrodermal activity (EDA), also known as galvanic skin response (GSR), measures the electrical conductivity of the skin, linked to the activation of the autonomic nervous system (or more precisely the sympathetic nervous system). By applying a weak current upon two electrodes attached to the skin, it is possible to measure the variations of voltage that are present between the measuring points (see figure 2.3c). When placed at specific locations on the skin, the measured electrical signals are affected by the sweat secreted by the glands that are found in the dermis.

What: Electrodermal activity signals inform about the activity of the sympathetic nervous system. Given its electrolyte composition, the sweat secreted by sweat glands has an impact on the electrical properties of the skin. This phenomenon, visibly monitored in voltage signals by means of electrical conductance (or impedance/resistance, conversely), facilitates the assessment of arousal effects. Arousal is the physiological response that stimuli such as emotional or cognitive stressors trigger. The measurement of electrodermal activity is usually decomposed in two major behaviours present and superposed in any skin response signal, i.e. the skin conductance (tonic) level, with slowly varying dynamics, and the skin conductance (phasic) responses, that exhibit relatively faster dynamics. In terms of signal analysis, this decomposition is accompanied by the assessment of characteristics such as the rate of detected EDA events, detection of onsets, and the characteristic rise and recovery times. 
Features: Onset instant; Skin Conductance Response (SCR) rise time; SCR 50\% recovery time; Event rate; Skin Conductance Level (SCL).

Where: EDA measurements use two electrodes to monitor changes in electric potential between two locations on the skin. Electrodes must be placed a few centimetres apart for differences to be relevant. The nature of the measurement technique and the phenomenon itself, makes hands and foot soles the most suitable electrode locations. On the hand, either palm or finger phalanges, most subject to skin sweating, are optimal for the monitoring of electrodermal activity. Foot (sole) placement, also affected by sweating glands, is not uncommon either in EDA measurements given that particular use cases or settings require access to hands for carrying out certain activities. For the alternative placements of the EDA sensors, such as forehead or wrist, the presence (or lack) of sweating glands remains a decisive factor in obtaining reliable measurements.

When/Frequency: Electrodermal activity is a slow physiological signal. Thus, sampling rate frequencies as low as $10 \mathrm{~Hz}$ allow a full representation of the skin conductance variations. Electrodermal activity peaks usually occur after few seconds from the exposure to a given stimulus (1-5 seconds).

Limitations: Electrodermal activity measurements use changes in electrical properties of the skin produced by sweating. Since sweating is not only triggered by arousal but also the human thermoregulation system, ambient heat and physical activity monitoring are aspects that limit the capabilities of EDA studies. In common practice, electrodermal sensors are usually prepared to obtain salient data from the most comprehensive userbase, providing relevant (measurable) changes regardless of the wide variety of sweating responses from subject to subject. However, it is not uncommon to find examples of subjects with either too high or too low skin conductance responses that complicate the measurements. Moreover, settings that involve an intense physical activity pose concerns on the electrode attachment and motion interference. The presence of power supplies and mains (power line) in the vicinity of the acquisition systems pose the risk of $50 \mathrm{~Hz}-60 \mathrm{~Hz}$ interference. With regard to feasibility, since traditional electrodermal activity studies rely on hands or feet electrode placement that compromises certain actions, attention needs to be given to the use case and activities that take place while monitoring, on a case by case basis. 


\subsubsection{Electroencephalography (EEG)}

How it works: Electroencephalography (EEG) measures the electrical activity of the brain, usually in a non-invasive way, from its outer layer. The acquired voltages correspond to ionic currents present in the activation of neurons (see figure 2.3d). This measurement, often done via scalp electrodes, provides electrical signals that allow the analysis of what is known as event-related potentials that link brain activity to specific stimuli or actions, together with the study of the predominance of certain frequencies in neural oscillations.

What: Electroencephalography signals inform about brain activity. The measured signals, usually acquired from different standard positions on the scalp, offer a complex overview of the brain (neural) activity oscillations. EEG combines the electrical monitoring of several areas of the brain, distributing electrodes (channels) along the head, giving rise to a wide range of signals representing neural activity at the different zones assessed. Although EEG is mostly approached from a multichannel perspective, few- and single-electrode EEG platform examples with simpler but limited access to EEG signals have gained attention in recent years. In terms of analysis, EEGs are traditionally studied from an event-related potential perspective, i.e. focusing on the changes in neural activity observed at the presence of certain stimuli or actions and from a spectral perspective, i.e. analyzing the frequency bands and corresponding oscillations that make up a given electroencephalography signal by means of frequency filters. EEG gives access to studying the power of the different frequency bands (alpha, beta, gamma, delta, theta, mu), linked to different brain functionalities as well as the onset detection of certain events that trigger neural activity (e.g. eye blinking, counting). Moreover, signal characteristics such as phase dynamics (e.g. phase locking between signals, phase coherence) are at the scope of EEG studies.

Features: Alpha band power; Beta band power; Delta band power; Theta band power; detection of events (e.g. cognitive efforts, eye blinking event, awakening);

Where: EEG measurements are acquired via several electrodes placed directly on standardised locations on the scalp surface. This is achieved by using either EEG caps, EEG headsets, meshes or, alternatively, single EEG electrodes. Importantly enough, electrodes must be in close contact with the skin, often requiring making contact between the subject's hair, the use of caps or headbands that press and ensure the contact, or supplementary gel that facilitates electrical contact in the 
areas of interest. One of the electrodes, used as a reference, allows to derive the electrical activity present at every zone under study. In the case of singlechannel configurations, consisting of two electrodes and a reference one, a bony or electrically inactive area is chosen as a reference.

When/Frequency: Electroencephalography signals are relatively fast. Given the fact that the frequency bands of interest differ remarkably, the targeted frequency band should guide the choice of an adequate sampling rate frequency. Considering the highest frequencies (gamma band) in event-related potentials, and trying to make a choice that prevents frequency aliasing, sampling rate frequencies of $200 \mathrm{~Hz}-250 \mathrm{~Hz}$ should cover any EEG relevant event.

Limitations: Electroencephalography provides complex biosignals, often involving the assessment and integration of several channels (several electrical signals) concurrently. This complexity requires the application of effective noise removal techniques. The fact that EEG platforms are portable and relatively non-invasive has contributed to the widespread use of the technology over other brain monitoring techniques or interfaces [Nicolas-Alonso and Gomez-Gil, 2012], regardless of the EEG limited spatial resolution. As the technologies progress, the quality of signals acquired nowadays is improved, yet subject to noise. Power supplies (mains, powerline) can potentially interfere with the signals. In terms of electrical artefacts, EEG is prone to movement or muscle interference mainly related to eye or head movements, making proper grounding a key aspect. Although the biosensing market has made great advances in creating low channel EEG platforms that present less obtrusive form factors, wearability comes at the cost of lowered spatial resolution and precision due to the reduced number of source channels as compared to lab-based EEG headsets. Moreover, some commercial applications choose designs that do not make the raw data or the processing steps evident or fully available.

\subsubsection{Inertial Measurement Unit (IMU) signals}

How it works: An inertial measurement unit (IMU) combines accelerometers, gyroscope, and magnetometer sensors to measure acceleration, rotation, and magnetic field in the three orthogonal directions of space. Used as a body sensor, it informs about its movement. Built upon microelectromechanical systems (MEMS), accelerometers use the displacement of a tiny mass surrounded by capacitors to measure 
proper acceleration. Gyroscopes, use the Coriolis displacement of two opposite oscillating masses to measure the rate of rotation (or rotation speed). Magnetometers are capable of measuring the magnetic field by means of magnetoresistance changes, informing about the orientation. Hence, IMU signals depict voltage variations corresponding to acceleration changes measured by accelerometry (ACC), rotational speed changes measured through gyroscopes (GYR) and magnetic field fluctuations measured by magnetometers (MAG), throughout a given dimension in space (see figure 2.3e).

What: IMU signals inform about the properties of the movement of the body which they are attached to, such as orientation and changes in speed. The measurement of accelerations and rotations, together with orientation, helps the researchers assess the existing movement patterns. Characteristics such as tilt (orientation), changes of direction, or number of repetitions in a given movement pattern (e.g. steps) are usually addressed. This makes possible the understanding of gait, posture, the dynamics of specific gestures or movements, as well as the detection of undesired movement patterns. Moreover, the measurement of movement properties is crucial in areas like ergonomics research and in estimating the metabolic equivalent of tasks addressed in physical effort studies. In terms of features and processing, information of interest can be fundamental frequencies or rates, maximum angles (movement range), and maximum accelerations are of interest.

Features: Metabolic equivalent of task (MET); Angular position (with respect to the ground plane); Footstep instants; Maximum acceleration; Maximum angular velocity. More advanced processing of multiple sources integrated can delve into gesture recognition, gait assessment, and other movement pattern analysis.

Where: The use of IMUs is extensive in monitoring navigation systems, present in many vehicles, and the devices are nowadays part of the set of sensors that mobile phones are equipped with. IMUs used as the body movement tracking systems work through the different sensors placed on the body part that is subject to study. This is typically the case of limbs and joints, shoulders, hips, or head, among others. Just like in the case of mobile phones, the wide use of IMUs has fostered the appearance of gadgets and wearables equipped with this monitoring technology (e.g. helmets, head-mounted displays, handles, controllers, footwear) that can monitor movement properties without necessarily having to attach sensors on the body. 
When/Frequency: Depending on the target action or movement to monitor, the needed sampling rate frequencies for signal acquisition may vary. While activity tracking and classification studies have succeeded in implementing the use of IMUs in a very low sampling rate frequency (less than $50 \mathrm{~Hz}$ ) there is the risk that, depending on the use case, fast vibrations or changes in rotation are not fully described. Higher values, e.g. $100 \mathrm{~Hz}$, may be a better tradeoff between sensing capabilities, data processing, reliable signal representation, and data storage needs.

Limitations: Despite the fact that inertial measurements are becoming less and less intrusive through the different forms in which monitoring devices are presented, IMUs present some limitations. Given the fact that inertial measurements combine different sensors, working on the 3 spatial dimensions, IMU signals are relatively complex to integrate. However, signal processing and activity recognition algorithms that build upon this integration is a developed area of research in itself. Magnetometer measurements, in particular, are prone to disturbances caused by the environment. With regard to accelerometers, measurements often accumulate drifts that compromise the estimation of trajectories.

\subsubsection{Breathing activity}

How it works: Respiration (or breathing) sensors monitor the inhalation-exhalation cycles of breathing, i.e. the process to facilitate the gas exchange that takes place in the lungs. In every breathing cycle, the air is moved into and out of the lungs. A breathing sensor uses either piezoelectric effects on bendable wearable bands or accessories (one of the most predominantly used technologies), respiratory inductance plethysmography on wired respiration bands around the thorax, microphonics on the nose/mouth airflow, plethysmographs (measuring air inflow) or radiofrequency, image and ultrasonic approaches. A review on breathing monitoring mechanisms is found at [Massaroni et al., 2019]. For piezoelectric breathing sensors, thoracic or abdominal displacements (strain) produced in breathing cycles bend a contact surface that converts resistive changes to continuous electrical signals (see figure 2.3f).

What: A breathing signal informs about the respiration dynamics, i.e. the dynamics of the process mediating gas exchange in the lungs, as well as supporting sound and speech production. The monitoring of the fundamental function of breathing 
brings in the assessment of breathing cycles and rates which in turn allows the study of apnoea-related problems (involving breathing interruptions), oxygen intake, metabolism of physical activity, and the effect of cognitive or emotional stressors in breathing. In terms of analysis, breathing cycles are studied using breathing rates, the maximum relative amplitude of the cycle, inhale-exhale volume estimation, inhale-exhale duration, and inspiration depth, that allow the characterisation of several breathing patterns.

Features: Respiration rate; Inspiration duration; expiration duration; Inspirationexpiration ratio; Inspiration depth;

Where: A piezoelectric breathing sensor is usually located on the thoracic cavity or the belly, using a wearable elastic band. With adjustable strap and fastening mechanisms, the sensor can be placed slightly on one side where bending is most relevant, optimizing the use of the sensor range. These kinds of sensors, allow both the study of thoracic and abdominal breathing. With the development of conductive fabric, breathing sensors are making its way into the smart garment market in the form of T-shirts and underwear bands.

When/Frequency: Breathing is a relatively slow biosignal, with breathing rates often below 20 inhale/exhales per minute. A sampling rate frequency as low as $50 \mathrm{~Hz}$ is sufficient to capture the dynamics of respiration.

Limitations: While piezoelectric breathing sensors are prominent given the low cost and form factor advantages of wearable sensor platforms, deviations in placement have an effect in the relative range of the response signal. Movement artefacts, most relevant when physical activity is present, are a common source of noise. Respiration sensing techniques like the respiratory inductance plethysmography, compensate the highly localised piezoelectric approach with a sensor capturing the general displacement of the whole thoracic cavity, yielding a signal less prone to movement artefacts. The monitoring of breathing cycles is usually accurate, although the exploration of effects to be used as voluntary inputs in interactions, such as holding the breath, are not easily captured. 
PART I 



\section{Chapter 3}

\section{Wearable health: Simplistic processing and other advances}

The recent development of wearable fitness and health tracking systems, i.e. personal sensing that monitors physical activity, behaviour and physiology, has been met with enthusiasm. The promise of personal tracking systems ranges from:

(a) Extending the research possibilities beyond the labs, with huge impact on augmented behavioural metrics or efforts entering physical and mental wellbeing domains

(b) Offering the opportunity for people to know themselves better, a movement called the Quantified Self

(c) Providing healthcare access to broader populations at a lower (more affordable) cost for societies

(d) Exploiting personalisation in a way that potentially unveils a new rapport between people and their own health, improving adherence to treatment, to say the least

The advent of affordable, high quality, personal sensing, is found in a context where the parallel development of high performance computing and artificial intelligence faces a data-driven society that offers unprecedented technological opportunities. From the iPhone, to the FitBit, new ecosystems of interconnected 
tracking devices, within the Internet of Things (IOT), are laying the foundations for business and research that will capitalise on machine learning (ML) progress. Tracking systems, however, with such a giant market behind, face the challenges of not only showing capabilities but proving their effectiveness, addressing what makes them valuable and trustworthy in relation to our expectations [Merrill et al., 2019] and unveiling how users interact with them, for instance capturing acceptance vs abandonment trends [Clawson et al., 2015]. Met with scepticism, it is the role of the research community to ensure that such profit translates into a societal change that brings broader access to health, a more inclusive and environmentally respectful progress, a deeper understanding of the human condition and a strengthened active role of the subject.

\subsection{Making biosignals available: Biosignal monitoring interfaces}

Biodata encompass a broad range of sources of body information, from cyrcadian, sleep and hormonal cycles, to metabolic activity (food or substance intake, digestion and excretion), mainly physiological, but also social contact and physical activity, among others. A particular form of biodata where records are concatenated tightly through time is that of biosignals (as introduced in 2.1.2). In the context of physiological monitoring platforms, it is interesting to count on tools that help track biosignal characteristics dynamically, i.e. delivering insight through changes measured over time. As personal sensing platforms build more and more on biosignal acquisition systems that already provide information continuously, the relevance of knowing how features change or even detecting events not long after they occur becomes more evident. Elaborate, processed information takes a main role. Raw biodata alone is no longer the main commodity, when working with these biosensing technologies.

\subsubsection{The problem}

The problem that is addressed in this research, which has been dubbed "Making biosignals available", consists in the following: In order for biosignals to be useful for design, wearable biosensors' data interfaces, tutorials, features and processing mechanisms that go beyond raw data storage are needed. As a way to challenge 
what is possible, or in other words how biosignals and its processing are used to infer relevant information, attention is firstly drawn on wearable health. The application of biosensing technologies to the domain of health has accompanied the rise of biosignal acquisition devices and motivated their improvement. By taking a perspective more aligned with biomedical engineering, machine learning and signal processing, the recognition of directions for future development is sought.

Before posing the question on how to make biosignals available for interaction design and human-computer interaction research, the possibilities of making biosignals available to developers are addressed, translated in biosignal processing interfaces, novel or simple processing algorithms and richer biosignal features.

\section{How can biosignals be made available for development, through data processing interfaces, novel algorithms and features?}

Focusing on wearable health, interesting intersections can be unpacked, as it will be shown. Real-time monitoring and features are not only relevant within hospital settings, but also in out-of-the-lab health platforms, since accessing immediate information means working towards making the most informed decisions in the many settings where tracking technologies are present nowadays. A personal tracking system that delivers information in a fast manner, is not necessarily opposed to the longitudinal analysis of data - either by aggregation of data points or via lower sampling or data processing rates- but complementary. This fact, we will see, has implications in health monitoring, as it is the case in the anomalous heartbeat classifiers able to raise a flag when a physiological condition of risk is detected (section 3.3.1), in the case of timely predicting a fatal health breakdown such as sepsis (section 3.4), in the case of continuous fatigue tracking as presented in section 3.5.2, but also in making biosignals available for the design of interactions. At PLUX, a multimodal biosignal acquisition platform manufacturer, there is the commitment to make biosignals more available. The work on the democratisation of physiological monitoring achieved by the hardware efforts devoted to low-cost and reliable monitoring platforms like BITalino [BITalino, 2013, Batista et al., 2019] and the development of higher-end, wireless, physiology tracking platforms with form factors that pave the way for biosignal research beyond traditional hospital settings such as biosignalsplux [PLUX S.A., 2017] are core development lines within this active field. Besides, software is an important asset of the biosignal monitoring ecosystem that is being created. A whole team of 
biosignal engineering developers is focused on maintaining different application programming interfaces (API) and software that serve the purpose of managing the biosignal acquisitions and revealing biosignal features by means of processing algorithms that encourage the user to appropriate and further utilise the information contained in the measured biodata. The initial filters and processing mechanisms made available are a stepping stone for those who want to further understand electrophysiology or develop customised processing algorithms that better suit specific purposes.
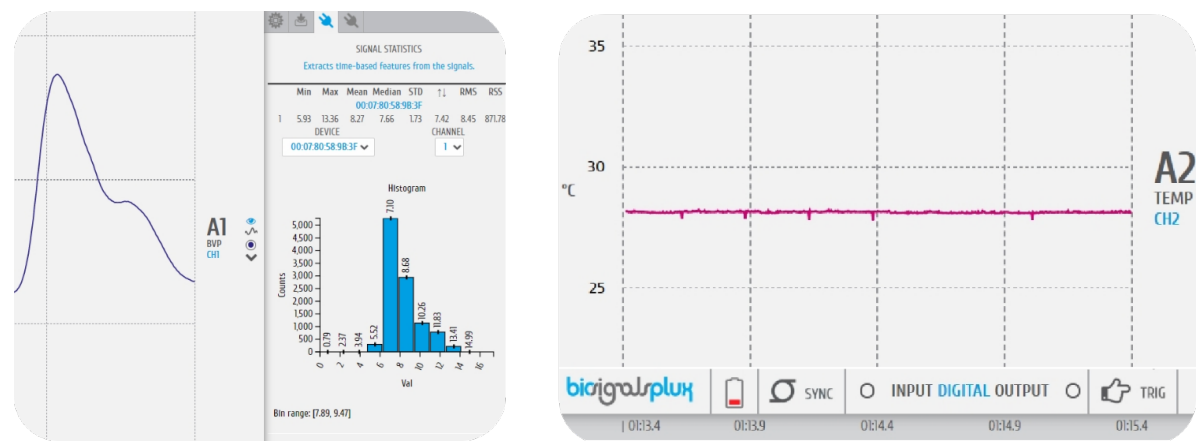

Figure 3.1: PLUX OpenSignals software implementation of histogram (statistics) and battery monitoring assets

In the course of the doctoral research presented here, there have been opportunities to collaborate in the development of software tools that render biosignal information more available while ensuring users are given the control options necessary for the planning of acquisitions of use within biosignal research. Some examples of these, that counted on this thesis contribution, are biosignal histograms which translate signals into statistical measurements that expose characteristics such as ranges, averages, maximum and minimum values, among others. Furthermore, biosignal platform interface assets such as the monitoring of the battery lifetime are of relevance for the planning of acquisition sessions for research studies (see fig. 3.1).

The biosignal acquisition interface, crucial for the definition of acquisition parameters, the thorough analysis of signals during and after acquisition, and the assessment of undesirable effects has been used throughout the $\mathrm{PhD}$ research path. One example is the characterisation of movement artefacts present in blood volume pulse (BVP) signals obtained by customised wrist biosensing platforms 


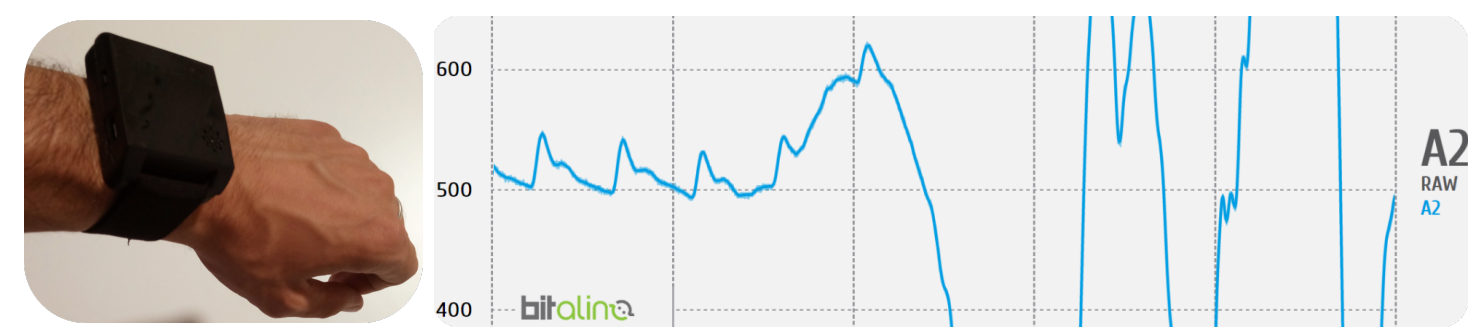

Figure 3.2: Study of blood volume pulse (BVP) noise in a custom-made wrist PPG sensor

using photoplethysmography (PPG).

From the open source perspective, other projects undertaken at PLUX are worth mentioning. In the context of BITalino, a platform much more aligned with community-supported development - as it is the case with the open source tools such as ServerBIT [BITalino, 2017] -, the organisation and hosting of introductory workshops on Do it yourself (DIY) biosensing led to the preparation of a collection of Hands-on code snippets (fig. 3.3) that guide the user into understanding, visualising and controlling the biosignal acquisition sessions [BITalino, 2018].
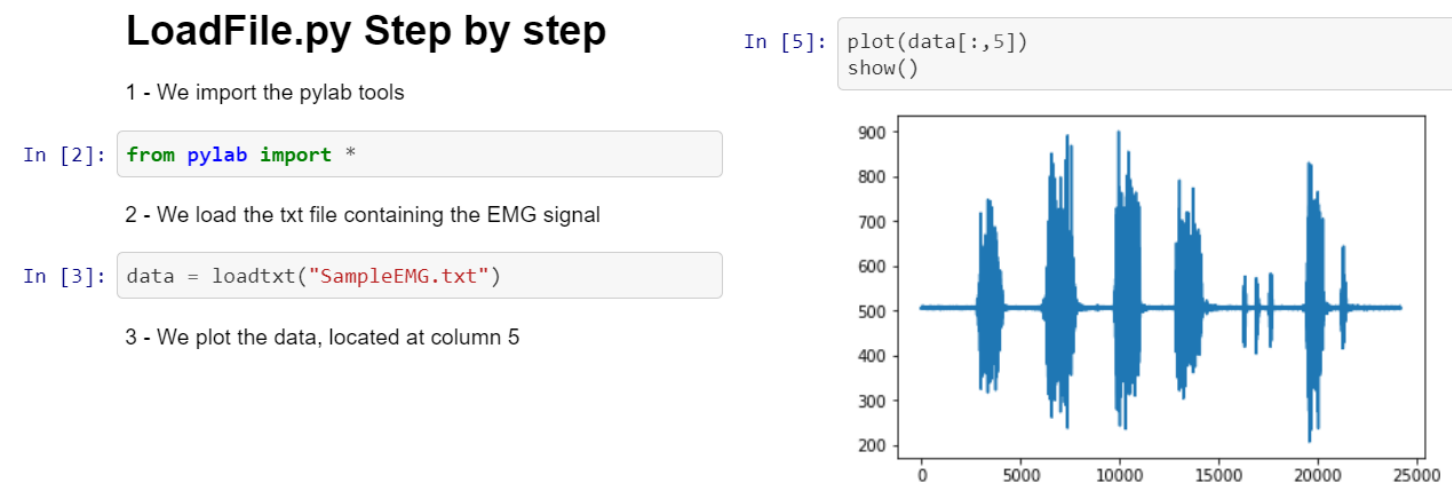

Figure 3.3: Example of open source BITalino Hands-on coding tutorials for biosignal acquisition and processing sessions (Python Jupyter Notebooks)

This collaboration of ours, together with Prof. Hugo Gamboa, took place during this thesis context and built upon the vast work undertaken by Prof. Hugo Silva and the BITalino team, who complement learning material with video tutorials that have become a staple of making biosignals available. It is worth noting that, 
recently, researchers at PLUX have taken those initial steps towards a learning environment for physiological tracking and extended it throughout the different acquisition platforms they manufacture, in an honest and open access effort to be a companion in the process of learning from - sharing and working with - our own physiology (see the biosignalsnotebooks [PLUX S.A., 2020, Varandas et al., 2020] project).

\subsubsection{Health and Machine Learning: The case for simplistic machine learning}

In this section, health research applications or goals are taken in order to provide a context in which machine learning uses have found a broad adoption. The discussions presented build on previous research conducted in the field of computationally undemanding neural networks for (health) time series processing [Ortín et al., 2019, Alfaras et al., 2019a] and unpacks current studies of this thesis, focusing on extending the application of simplistic neural network paradigms [Alfaras et al., 2019c], the use of real-time biosignal data processing and visualisation [Ramos et al., 2018], and the novel combination of features to enable the recognition of more elaborate physiology patterns [Ramos et al., 2020].

It is incontestable that deep neural networks (DNNs) and their recent success in many fields have revolutionised - and helped rebrand - artificial intelligence. From self-driving cars, to speech recognition or the prediction of plant disease in favour of an increased agriculture efficiency. The increasing amount of labelled data that researchers count on nowadays and the dramatically improved computing capabilities that exploit parallelism to circumvent the long announced end of Moore's law are to blame. These are the fertile grounds that make deep learning flourish, allowing a process of brain-inspired learning by example that lets scientists dream of the era in which technologies outperforming human capabilities will augment the human in tackling some of the great challenges of our times. Artificial neural networks (ANNs), though, comprise a wide spectrum of developments, wider than deep learning. Neuroscience, the science that is called to be the science of the century is still at early stages in understanding consciousness, thought and memory. In the development of artificial neural networks, while the inspiration on biology had been a major driving motor (von Neumann, Hopfield), science at some point benefited from disregarding neural limitations, such as dropping the rejection 
of backpropagation on the grounds of not being neurally plausible. A vast range of possibilities stood in front of ANN researchers. The unbounded development of ANNs took many forms, competing in pattern recognition or classification problems against now traditional machine learning approaches (random forests, support vector machines, etc.). Machine learning comes in many flavours. All of them offer different trade-offs and come with intrinsic strengths and limitations. Could personal sensing platforms harness the potential of the more simplistic machine learning paradigms?

In this chapter, the thesis focuses on 3 case studies that strengthen wearable health research and inform research directions for biosignal interfaces:

1. Echo state networks and arrhythmia. When alternative, simplistic recurrent neural topologies compete with the state-of-the-art algorithms (section 3.3)

2. Health records, Echo State Network morphology processing capabilities and heterogeneous data. Can a network supplant a doctor? (section 3.4)

3. Beyond classification. The move to real-time processing and the definition of new metrics (section 3.5)

\subsection{Materials and methods for the biosignal developer}

Biosignals and reliable acquisition platforms have been essential throughout the whole research presented in this thesis. The software and data handling platforms introduced in section 3.1 have also played an important role in seeing, understanding and interpreting biodata. In this section, however, an understudied tool that offers low-demanding computational requirements and promising physiology processing capabilities is presented. This is the case of Echo state networks, a particular recurrent neural network that does not usually fall within machine learning paradigms traditionally adopted and covered in reference texts.

\subsubsection{Echo state networks (ESN)}

Recurrent neural networks (RNNs) [Lipton et al., 2015] are artificial neural networks that incorporate recurrencies between computing neuron nodes, i.e. where 
links or connections allow information to cross more than once a given node or set of nodes throughout time. In other words, RNNs model temporal dependencies or sequentiality in an explicit manner. As opposed to standard neural networks, recurrent network states are not discarded once used, and part of the information is kept within the system, influencing further states. Within RNNs, the apparently unbounded number of steps at which information can spread or be retained is said to be behind the success of computationally expensive architectures such as the Long Short-Term Memory, a relevant ingredient of successful Deep Learning examples tackling handwriting, language/voice recognition and simultaneous translation among other machine learning problems concerned with data of sequential nature.

Alternatively, efforts towards simplistic recurrent neural network paradigms have seen in recent years the emergence of examples such as Echo State Networks (ESN) [Jaeger, 2001, Jaeger, 2007], garnering a relative success in machine learning tasks. ESNs emerged in the context of dynamical systems in the early 2000s, developed independently and simultaneously as the Liquid State Machines (LSM) from computational neuroscience, and are nowadays subsumed under the name of Reservoir Computing (RC). We use the RC and ESN terminology interchangeably. ESNs are RNNs that follow particular setups which, to some extent, avoid the computationally expensive update iterations and the growing number of optimisation parameters. One of the advantages that ESNs present, is in fact the simplification of the training process (in this case only done for the readout weights), given that optimisation is reduced to a few parameters. An echo state network, must satisfy the following properties:

- Consistency or approximation: 2 instances of similar information provided to the reservoir system must also present similarities in the nonlinear response space.

- Memory (fading, echo state): As a recurrent neural network, an ESN must ensure that couplings between nodes take place, so that temporal feedback is maintained within the nonlinear system of responses. This is, in other words, equivalent to stating that information is leaked between nodes or consecutive responses. In turn, this feedback or memory property needs to fade away for very long iterations, as information states distant in time and completely unrelated should avoid interference. 
- Separability: 2 instances of similar information provided to the reservoir must sufficiently differ so that responses can be interpreted as different and used as computational resources. A properly tuned separability should leverage the dynamic range of responses intrinsic to the reservoir.

In terms of elements, the setup of ESNs [Lukoševičius and Jaeger, 2009] involves:

(a) A network that is randomly created and whose internal connections remain unchanged;

(b) Input signals that excite the network, which maintains in it a nonlinear transformation of the input history;

(c) A linear combination of the input excited reservoir generates the output signal, based on the weight obtained via simple output regression using the target outcomes.

The fact that the network's internal connections are kept constant simplifies significantly the process of training. This has motivated the emergence of a wide range of reservoir computing implementations and applications drawing upon physical or analogue computation paradigms such as oscillatory-mechanical, fluidic, electrical, or photonic ones. Among target tasks for reservoir computing there are classification or recognition goals, signal-information recovery, or timeseries prediction. Examples of applications include, but are not limited to, e.g. a memristor-based reservoir computer for digit recognition [Du et al., 2017], a photonic reservoir counting on a delay-coupled semiconductor laser for spoken digit recognition [Brunner et al., 2013], etc. The idea behind the application of ESNs is to leverage the temporal information present in the data, e.g. making the network learn the characteristic response dynamics of the sound " $n$ " in the word "one" as different from that of the word "ten" when feeding spoken digits to the system.

The structure of an echo state network as seen in fig. 3.4 is that of a recurrent neural network, where internal connections are randomly set and fixed. First, a nonlinear mapping takes place in the input layer. Network nodes implement fixed links (with recursivity) within the reservoir $R$, establishing the feedback of information. Finally, the echo state is read and mapped onto outputs $(Y)$ thanks to the output layer weights $(W)$. 


\section{Echo state network}

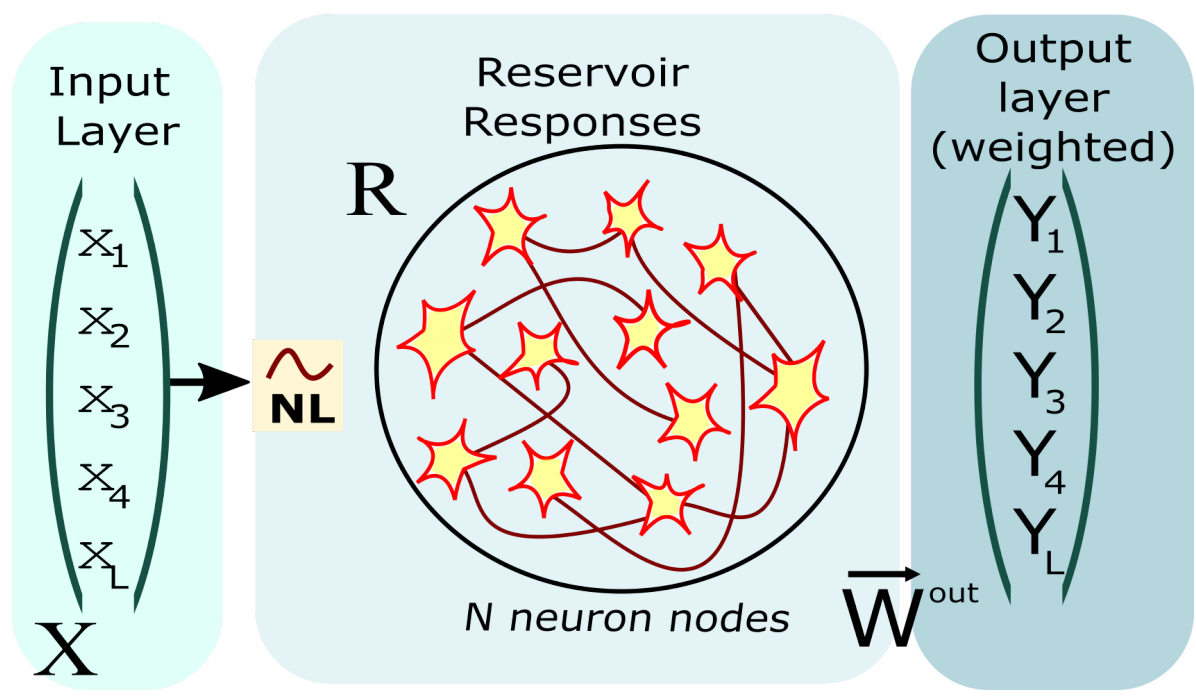

Figure 3.4: ESN structure, with the input layer, fixed reservoir and weighted output layer

\section{Training the echo state network}

The training procedure of a reservoir computer starts by providing a set of input data to the network. Data inputs are scaled and nonlinearly mapped to the resorvoir. Sigmoidal functions - in particular hyperbolic tangents - are usually chosen as nonlinear neural activation functions. The reservoir, implements its internal node connections, with memory being leaked among connected nodes as iterations unfold. A readout of the states is performed. The output weights that minimise the mean square error, i.e. the difference, between the target outcomes and the measured outputs at readout would be the optimal weights.

In terms of formulation, the reservoir system can be represented as follows:

$$
\mathbf{r}_{i}=F\left(\gamma \mathbf{W}^{\mathbf{i n}} \mathbf{X}_{i}+\eta \mathbf{W} \mathbf{r}_{i-1}\right),
$$

The reservoir state $\mathbf{r}_{i}$ at iteration step $i$ is the nonlinear activation pattern $F$ that results from adding up two terms: the combination of input $\mathbf{X}_{i}$ nonlinearly mapped by the input weights $\mathbf{W}^{\text {in }}$ and scaled by a $\gamma$ factor, together with the feedback reservoir response $\mathbf{r}_{i-1}$ mediated by internal connections $\mathrm{W}$ and feedback or memory strength factor $\eta$. Usually, having a sparse connectivity between nodes is 
thought to be responsible for the resulting rich dynamics of the network [Jaeger, 2007]. The reasoning is that sparsely connected nodes lead to a loosely coupled system that exhibits a large variation of states. This rationale, though, has been challenged by research reporting that fully connected reservoirs work as well as sparse counterparts. However, a practical benefit of sparse connectivity is seen in terms of computational cost, as constant rates of connections per neuron yield a linear scaling on computational complexity.

In attempting to explore alternative simplistic approaches - in terms of modelling and computational cost - to solving tasks of biosignal processing or classification nature, Echo State Networks stand out, not only provided the fixed internal reservoir structure, but also given the existence of simple topologies for which recent research has reported significant performance on different time series prediction tasks [Rodan and Tiňo, 2011]. Inspired by this work, promising capabilities are seen in simple cycle reservoirs, referred here as a ring topology echo state network. Experimental works on hardware implementations of reservoir computing that leverage single computational elements (nodes or neurons) multiplexed in time, lie close to these paradigms, as it is the case of [Appeltant et al., 2011, Soriano et al., 2015]. The approach of the simple cycle reservoir, consisting on having internal connections of fixed and equal intensity, is adopted. Input-to-reservoir connectivity is full. In this paradigm, neurons form a ring "non-random" fixed topology. The internal weight square matrix $\mathrm{W}$ exhibits nonzero single fixed absolute weights on the lower subdiagonal and the upper-right corner, which for a network of $N$ neurons takes the form of

$$
\mathbf{W}_{n+1, n}=\mathbf{W}_{1, N}=c
$$

Keeping the notation of eq. (3.1), the value of $c=1$ is chosen, as the feedback strength is controlled and optimised via the feedback parameter $\eta$. A difference taken with respect to the simple cycle reservoir proposed in the literature is that of limiting the symmetrical range from which input weights are drawn but not setting them as fixed and equal throughout the network. The chosen network, hence, does not optimise a shared input weight. This step, although maintaining input scaling factor optimisation, is taken as a way to address the possibility of combining ensembles of reservoirs or ESNs as a more general recipe or alternative to obtain relevant performances rather than optimising a shared constant input weight that does not necessarily exploit the dynamical richness that randomly 
assigned weights give to the network.

\section{Solving an ESN system}

If one looks at the reservoir formulation and the desired use of the classification system to be developed, the following equations help us understand the classification and training mechanisms:

$$
\hat{\mathbf{y}}=\mathrm{W}^{\text {out }} \mathbf{r},
$$

Here, an echo state network that works as a classifier works in the following way: When inputs are fed into a high-dimensional nonliear dynamic system, i.e. the reservoir, network responses $\mathrm{r}$ are recorded. If properly trained, a simple product by output weights $\mathbf{W}^{\text {out }}$ should be able to provide an output class label $\hat{\mathbf{y}}$. If we think of the system in terms of matrices encoding several inputs $Y$ and a collection of reservoir network responses $R$, we can manipulate the equations to present them as a linear system to be solved:

$$
\mathbf{y}=\mathbf{R} \times \mathbf{W}^{\text {out }} \rightarrow \mathbf{R}^{*} \times \mathbf{y}=\mathbf{W}^{\text {out }}
$$

In order to find out the output weights $\mathbf{W}^{\text {out }}$, one can make use of the pseudoinverse matrix of the echo state network responses $\mathbf{R}^{*}$ and the known labels used for training, $\mathbf{y}$. A trick to transform the system into a less computationally demanding one consists in using the normal equations [Lukoševičius and Jaeger, 2009], which by means of transpose matrices reduces the dimensions of the main matrix to solve or invert in the system of equations:

$$
\mathbf{R}^{t} \mathbf{R} \times \mathbf{W}^{\text {out }}=\mathbf{R}^{t} \times \mathbf{y}
$$

The resulting linear system can be solved for the output weights $\mathbf{W}^{\text {out }}$, via decomposition approaches or applying pseudoinverse matrices:

$$
\mathbf{W}^{\text {out }}=\left[\mathbf{R}^{t} \mathbf{R}\right]^{*} \times \mathbf{R}^{t} \times \mathbf{y}
$$

\subsection{Echo state networks and arrhythmia When alternative, simplistic recurrent neural topologies compete against state-of-the-art algorithms}

The fact that high performance computing has allowed the transformation of data centres, now cementing cloud computing services offered worldwide, with 
an agenda that targets the deployment of the infrastructure that will render a much more interconnected society (e.g., via the Internet of Things IoT and the advent of $5 G$ ) is accompanied with the enthusiasm of those who have witnessed the advances in computational power. With higher computational power, though, come higher transmission demands. In a world increasingly filled with devices, and in particular in wearable health, we have the opportunity to impact, from the way that data processing algorithms are implemented, and rethink the role that these devices will play in communications. In this context, the inspiration to seek simplistic, yet powerful, algorithms that devices could exploit on their own - or edge computing - emerges, far from more complex (deeper) artificial intelligence paradigms. Such is the case of echo state networks (ESNs), presented in section 3.2.1.

\subsubsection{Holter ECGs and heartbeats of different origin}

As a first step to enter wearable health, or more precisely, the improvement of machine learning applications on biosignal processing aligned with wearable and out-of-the-lab developments, the problem of cardiac arrhythmia is addressed.

Electrocardiography (ECG) (see section 2.1.4) has been a technology staple within the medical practice since its firsts uses in the late 19th century and early 20th century introduced by Nobel prize winner Willem Einthoven. Traditionally, an ECG signal is characterised by what is known as the P-wave, the QRS complex and T-wave, corresponding to a cycle of atrial depolarisation, ventricular depolarisation and ventricular repolarisation, respectively - i.e. a heartbeat. Voltage differences are drawn from electrode pairs located at specific regions of the body, capturing what is called an ECG lead. Standard electrocardiography carried out at medical facilities incorporates the assessment of several leads - typically 12 - by means of setups with multiple electrodes that yield a richer picture of the electrical nature of the heart - for an introduction to normal ECG leads and axis, i.e. the direction of the electrical flow within the body frontal plane, see the work of [Goldich, 2014]. Abnormalities in time (rhythm), amplitude or waveform across leads is what the trained eye of the cardiologist tries to spot when assessing ECG activity and looking for arrhythmia. The problem of detecting arrhythmia in ECG signals falls under the category of pattern recognition. With the development and popularisation of the ambulatory Holter ECG monitoring device in the second half of the 20th century, 
portable ECG approaches started to gain prominence in health and research. Evolving hand in hand with the psychophysiology sensor industry, incentivised by the miniaturisation and ubiquity of digital tracking systems, multiple medicaloriented and research-oriented portable ECG platforms captain and populate the today's market of health wearables. While moving ECG monitoring and opening cardiac research out of the clinic has great benefits - e.g. broader technology acceptance, low-cost, longitudinal, larger-scale studies - working with portable ECG tracking comes with challenges. One of such challenges is that of the trade-off between cutting on information richness, i.e. using fewer electrodes and leads, while optimising pattern recognition outcomes. Inspecting an ECG signal is, in essence, a task of looking for patterns or features that let one tell apart specific signals from their "physiologically normal" counterparts, or even examine the singularities that make an ECG stand out from a collection of samples within a given subject. In turn, this task, which uses temporal sequences as source material posits itself as a great candidate for machine learning approaches that leverage sequentiality, such as Echo State Networks.

Large databases such as those found at open access repository Physionet [Goldberger et al., 2000] (MIT-BIH AR, INCART) or the privately owned American Heart Association arrhythmia database [AHA / ECRI, 2003] are of immeasurable value for the development of artificial intelligence applications. Databases like these offer unprecedented biosignal processing development or improvement opportunities. The interest in ECG from Machine Learning communities is not new. In reviewing literature on the identification of arrhythmic heartbeats - i.e. heartbeats of abnormal origin - it is found that several approaches are used, e.g. ranging from Deep Learning, Support vector machines, Decision Trees or linear discriminant analysis (for a list of references and details, refer to [Ortín et al., 2019]).

\subsubsection{ML and intuition for wearable physiology}

Can a portable ECG monitoring device incorporate mechanisms to tell the difference from a healthy heartbeat and an abnormal one? Moreover, how can (mainly) morphologically-based classifiers compensate the minimal information that comes in a single/double lead ECG device?

Humans have wonderful pattern recognition capabilities that stem from the 
evolution of primary senses like sight and hearing. When a cardiologist pays close attention to ECG records, being printed from several source leads (standardised electrode placements) at once, spotting anomalies accounts to identifying differences in morphology along the signals, i.e. changes in time intervals, peak ranges, etc. Not only do doctors look for physiological anomalies, they look for signs that render a particular heartbeat unique within the context of a patient. When it comes to wearable ECG devices, most platforms provide a low number of leads, often relying on only one or two of them, with little or no guarantee that acquisitions correspond to standardised leads. In making the ECG signals more available for wearable health systems, the contribution of this work is twofold:

1. A simplistic machine learning approach, i.e. a ring topology echo state network, aimed at computationally-efficient classification temporal sequences suitable for portable monitoring systems is explored and assessed in terms of performance at classifying ECG signals

2. Alongside the chosen algorithm, the research aims to study different features leveraging the morphology of the signals, with the automatic processing of few ECG leads that aim at generalising well. Single lead approaches and patient variability are addressed.

Different types of arrhythmia, depending on the originating mechanism and place, as well as pace at which they unfold, are categorised in the clinical practice. In order to undertake a machine learning classification task, heartbeats are aggregated under the heartbeat types recommended by the Association for the Advancement of Medical Instrumentation (AAMI) [AAMI / ANSI, 2012] and assessed from a binary class of supraventricular vs. ventricular abnormal origin perspective. It is important to note that the choice of these aggregated classes constitute a good starting point to account for a simple binary class with respect to the zone where heartbeats are originated, i.e. normal and supraventricular ectopic as opposed to ventricular, but such representation is just part of a bigger picture of multiple heart dysfunctions requiring further analysis. In the paper [Ortín et al., 2019], the idea of template matching for the detection of hearbeats of ventricular origin is implemented.

Template matching, used previously in [Krasteva et al., 2015] consists in the creation of a class template, i.e., prototypical heartbeat of the class to serve as the 


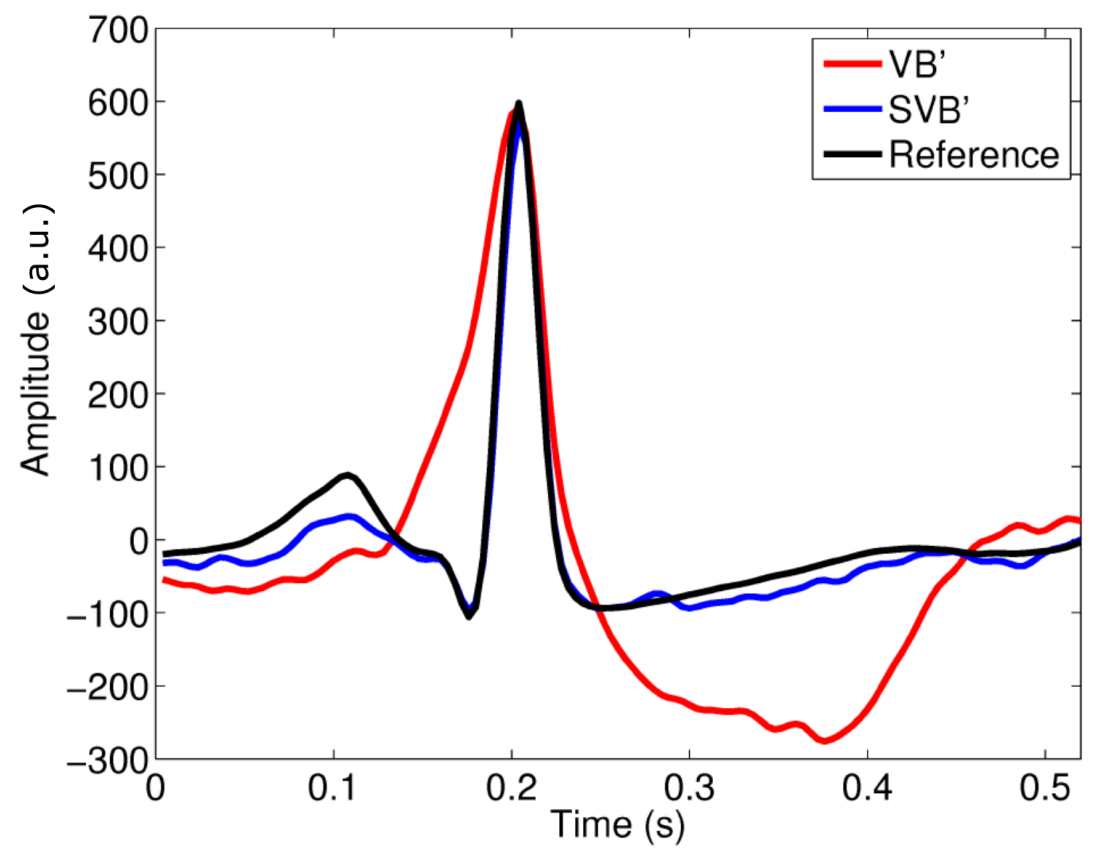

Figure 3.5: Example of a template sinus (normal) reference ECG heartbeat and their Supraventricular (SB') and Ventricular (VB') beat counterparts 
basis to be compared to. With a template reference per subject has the potential to reduced inter-subject ECG variability errors. Working with references renders the classification lead-independent or even suitable for the non-standard leads that may appear in wearable developments, as a class template can be developed in any of the leads. Under these premises, several correlations between heartbeat morphologies and those of the corresponding class templates are computed. Moreover, simple time-domain features such as updated interbeat period averages, neighbouring intervals or statistical measurements associated to them complement the template correlations to increase the performance of the classification.

The creation of a reference beat, requiring a 5-8 min. warm-up period, is done by relying on an intuitive morphology property. As data from different patients in handled, in order to capture the normal dynamics of a a subject, the reference needs to avoid being biased toward abnormal beats. For this reason, the process of building a reference consists of taking a buffer of heartbeats, computing their average absolute amplitude value, the average absolute value of their first derivative approximation and discarding the $50 \%$ of buffered heartbeats with the slowest dynamics, i.e. those which present a largest ratio between the two aforementioned properties. The reference beat is then updated throughout time, every 15 heartbeats, dynamically accounting for changes in activity. The system is trained with the strategy presented in equations 3.1-3.5. As an interesting result, the competitive results of the proposed ring-topology echo state network seemed to leverage the possibilities that information contained in single leads offer. Addressing inter-subject variability with a reference template seemed to capture morphology information in a relevant manner. Its success, that of an elaborated yet understandable feature, is likely attributed to the metric in the template building recipe, somewhat translating a visual intuition into a similarity measurement that is added to ECG features regularly used. But could ECG morphology be used by ESNs more explicitly or straightforwardly?

In the paper [Alfaras et al., 2019a], in another push on approaches with low computational costs suitable for wearables a simpler idea is explored. Can a classification primarily based on single lead morphology provide a ventricular heartbeat that competes with state-of-the-art results? The idea behind this work, is to probe whether simple ECG machine learning approaches can make use of raw signals rather than elaborated feature extraction methods. Drawing upon the previous success of ring-topology echo state networks for heartbeat classification, 
the same structure is proposed and fed with ECG wave datapoints as features. In order to train the reservoir, the set of chosen features will be fed to the system together with the expected output labels, so that a linear regression at the output layer can identify the best combination of output weights. The interesting part lies in the features. After standard filtering, peak detection and a simple scaling, a subset window of raw ECG datapoints around the QRS complex is provided to a reservoir classifier. An example of the minmax scaling can be seen in fig. 3.6.

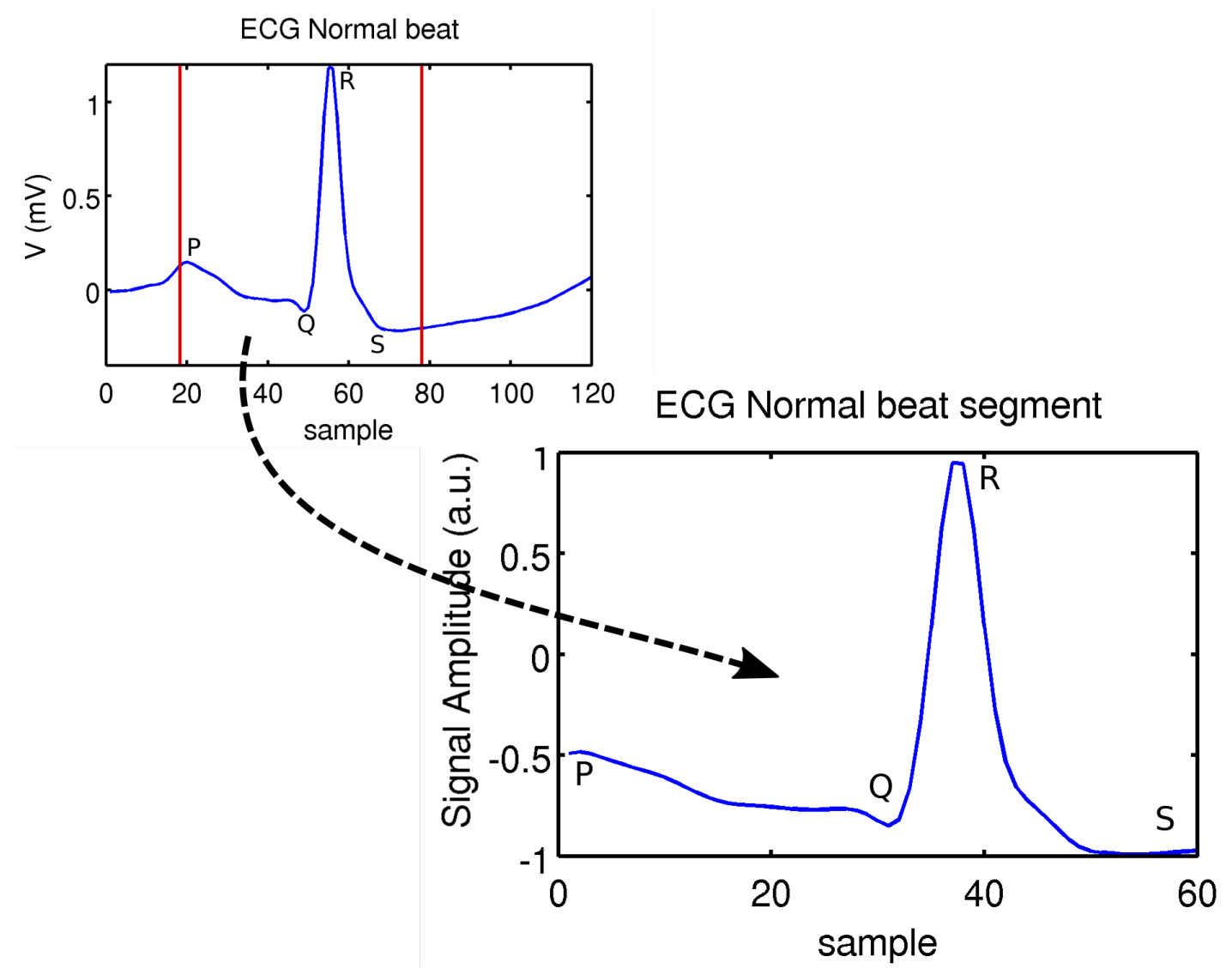

Figure 3.6: Average healthy heartbeat minmax scaling

These "features", perhaps deemed oversimplistic, together with basic peak to peak measurements are then mapped to the high-dimensional nonlinear space of an echo state network. Surprisingly, the ECG lead, the scaling, and duration about $240 \mathrm{~ms}$ centred on the QRS - successfully work at training a classifier in a standardised way. With this approach, the network responses are basically the result of exposure to ECG morphologies of different kinds. In the reported results, 
a fascinating aspect is worth highlighting: not only state-of-the-art performance on automatic single lead ventricular ECG classification is achieved, but approaches that train on a lead and test on another achieve relevant performances even for interchangeable databases. This result, although modest, paves the way for future research that aims to push the frontiers of generalisation. Of particular interest is the fact that physiology tracking out of the lab, by means of Holters or monitoring devices alike, often runs into single lead based paradigms - even nonstandard leads. Having a computer trained on a gold standard being applicable to in-the-wild use cases closer to real life is, to say the least, an encouraging scenario for future uses of wearable health monitoring systems. The study presented in [Alfaras et al., 2019a], also offers a look into using the parallelisation of computational steps involved in the training of the echo state network. Given the simplicity of the formalism of the reservoir equations (see eq. (3.1)), operations such as matrix multiplication and nonlinear mapping involved in the system preparation and the linear equation resolution are perfect candidates to exploit the computing capabilities of graphic processing units (GPU). Exploring a communication protocol deploying real-time data transfer from device to central unit to deliver classification of heartbeats on the fly does not fall under the scope of this research. However, it is easy to envision that use-case scenario. The training of algorithms with large databases is where GPU power may be exploited best. As exemplified in the paper, training a reservoir computer configuration with more than $10 \mathrm{~h}$ worth of filtered ECG is a matter of 2 seconds of GPU, meaning that different parameters explored during optimisation such as scaling factor, feedback strength, or reservoir size can be explored more easily. As a side note, it is important to note that moving away from the cycle [Rodan and Tiňo, 2011] recipe on fixed input weights, ours is an approach that leverages ensembles of classifiers with nonidentical randomly generated input weights, in a shortcut to reduce the parameter space while making the most of different nonlinear response patterns combined via several classifiers (increasing performance up to 30 ring networks in our example). 


\subsection{Health records, Echo State Network morphology processing capabilities and heterogeneous data}

Having been inspired by the success of echo state networks applied to ventricular heartbeat classification, a use case possibly contributing to the research on wearable health, this thesis embarked on a challenge launched by the Physionet on data from intensive care units (ICU). Part of the success of the properly trained networks at spotting heartbeats of ventricular origin could be attributed to the nature of the problem. ECG data is highly temporal, presenting rhythms and sequences that repeat over time. In this context, one can expect that algorithms suited for sequential information processing can leverage information and shed light on subtle differences between consecutive heartbeat sequences. When thinking of the simplest features of all, i.e. amplitude values capturing the biosignal morphology, the dependence between contiguous features is undeniable, as in principle two close samples would not exhibit great differences in value. A continuous signal such as ECG would not present leaps that are difficult to explain. These are behaviours likely captured by the nonlinear responses of the ESN network. What if the remarkable processing capabilities exhibited by simplistic topology ESNs on classifying physiological signals could contribute to artificial intelligence health problems based on ICU medical records? Would the rich morphology processing capabilities of a simple ESN still apply to highly heterogeneous data? The Physionet 2019 challenge [Reyna et al., 2020] presented a problem that differed significantly from ECG analysis. The topic revolved around sepsis, a prevalent issue in ICUs all over the world.

The challenge objectives are reproduced here: "Sepsis is a major public health concern with significant morbidity, mortality, and healthcare expenses. Early detection and antibiotic treatment of sepsis improve outcomes. However, although professional critical care societies have proposed new clinical criteria that aid sepsis recognition, the fundamental need for early detection and treatment remains unmet. In response, researchers have proposed algorithms for early sepsis detection, but directly comparing such methods has not been possible because of different patient cohorts, clinical variables and sepsis criteria, prediction tasks, evaluation metrics, and other differences. To address these issues, the PhysioNet/- 
Computing in Cardiology Challenge 2019 facilitated the development of automated, open-source algorithms for the early detection of sepsis from clinical data."

Sepsis is defined as "life-threatening organ dysfunction caused by a dysregulated host response to infection" [Singer et al., 2016]. This condition can be detected using physiological measures, such as, heart rate, temperature and laboratory measures of body fluids analysis. For patients in the ICU, sepsis prediction is compromised due to the effects of the prescribed treatment. For instance, drugs that help to attenuate the patient symptoms have an impact on physiological measurements (temperature, heart rate, etc.). In turn, mechanical ventilators do not allow to take the respiration rate into account in order to detect sepsis. Inspired by the successful application of ring topology echo state networks for the classification of biosignals of relevant temporal structures, in particular that of ventricular heartbeat classification carried out on ECG data, this thesis explored whether a similar network could tackle the prediction of septic shocks. This work was conducted in a collaboration with the Instrumentation lab (LIBPhys-UNL) at Universidade Nova de Lisboa. While the application of ESNs to ECG classification faced temporal signals where morphology was to be exploited, ICU health records pose several differences. The data provided by the challenge organisation consisted originally in physiological, laboratory and demographic measurements of more than 40000 patients from the ICU of two different hospitals. In the interest of fairness, and besides counting on a third unshared hospital dataset, the organisers sequestered data from the two hospitals where data was sourced from, so that no training could be performed based on samples that are to be used for testing and scoring only. Values were registered hourly, with missing data points marked with $\mathrm{NaN}$ (not a number). At any isntant in time, a Sepsis Label indicating the presence of sepsis is provided. Features accounted for 40 indexes tracked over more than $1.5 \mathrm{M}$ hours. However, $70 \%$ of those data points are NaN. Among samples, sepsis appeared only on $1.80 \%$ of the total. Since the scope of the challenge was sepsis prediction, the organisation created an ad hoc utility function that would penalise 
classifications too anticipated or delayed in time, prioritising timely classifications.

$$
U(s, t)= \begin{cases}U_{T P}(s, t) & \text { septic patient } \mathrm{s} \text { has a positive prediction at } \mathrm{t} \\ U_{F P}(s, t) & \text { healthy } \mathrm{s} \text { has a false positive at } \mathrm{t} \\ U_{F N}(s, t) & \text { septic patient } \mathrm{s} \text { has a negative at } \mathrm{t} \\ U_{T N}(s, t) & \text { healthy } \mathrm{s} \text { has a negative prediction at } \mathrm{t}\end{cases}
$$

The utility function $U(s, t)$ for subject $s$ at time $t$, takes the form of the different values that, according to time, reward true positive as compared to negative outcomes (TP, TN) and penalises false negatives (FN) significantly or false nefatives slightly $(\mathrm{FN})$. $6 \mathrm{~h}$ from onset is the time at which correct predictions are rated best and false negative oucomes start to reduce utility.

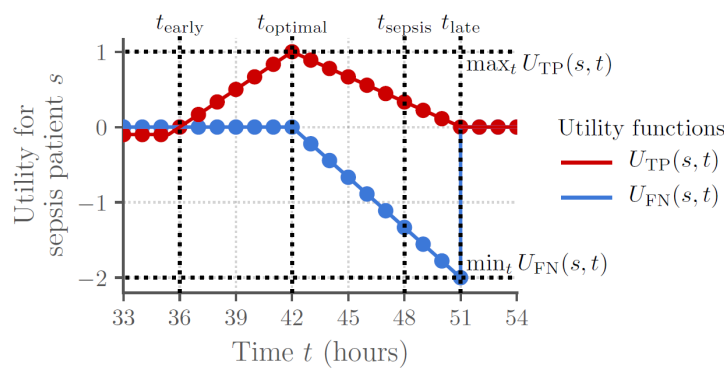

(a) Utility function for septic subjects

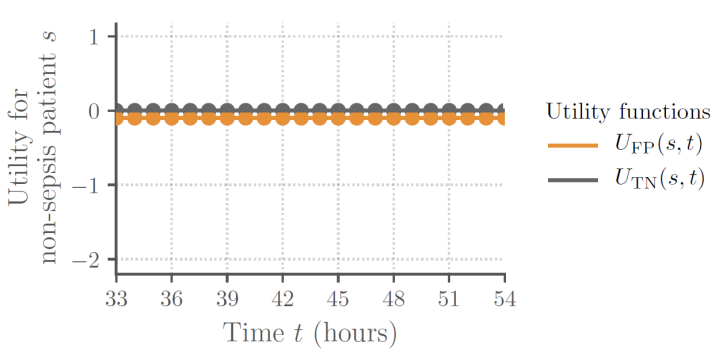

(b) Utility function for healthy subjects

Figure 3.7: Clinical utility rating function for septic prediction

Classification at the time when patients are labelled as septic by doctors would be of limited use or improvement for a condition that, too often, leads to complications and death when detected. Septic class labels had suffered a $6 \mathrm{~h}$ time shift in accordance to the objective of forcing algorithms to learn associating physiology data to a septic state before becoming apparent.

The first of the issues encountered was that of missing data (see fig. 3.8). In order to circumvent this problem and allow classifiers to process the data rather than discarding entire features or subjects, different substitution strategies were laid down:

1a Constant value replacement (using zero)

1b Constant value replacement (using a stored an average value) 
2 Random normal distribution value replacement around the average value of the feature

3 Interpolation between closest significant neighbouring values, with simple replication when no later values exist

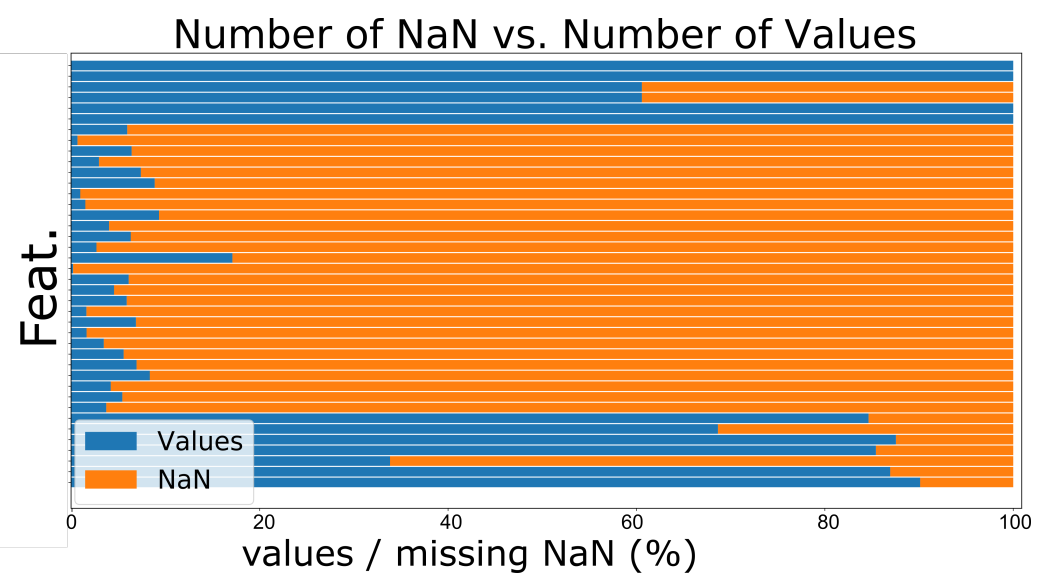

Figure 3.8: Sepsis dataset ratios of missing data (\% of not a number values, NaN) per feature.

After the unofficial phase of the challenge, with initial data processing approaches being tested, it was made clear that non-causality should be avoided at all costs. That meant that classifiers and processing steps that needed the full story of a patient, looking into the future in order to perform scalings, computing statistical moments or placing missing value replacements would present serious applicability in real-case scenarios. As opposed to the ECG classification problem, dealing with features that maintained a high temporal- and morphological- relation, the sepsis challenge consisted in mapping 40 -feature vectors onto a nonlinear response space, where links among features are not evident a priori.

\subsubsection{Training an ESN sepsis classifier}

Following a ring topology approach (see section 3.2.1), the 40-dimensional inputs are fed via an input mapping with uniformly distributed random weights and offsets (or biases) and a sigmoid nonlinearity activation, eq. (3.9), that transforms 
the scaled inputs and combines the current and previous state into a new response:

$$
\begin{gathered}
E s n_{n, t}=f\left(\gamma \text { Input }_{t}+\eta\left(W_{E s n} \times E s n_{n-1, t-1}\right)\right) \\
f(x)=\left(1+e^{-x}\right)^{-1}-0.5
\end{gathered}
$$

In the system equation (3.8), with the structure of eq. (3.1) $E s n_{n, t}$ is the state of neuron $\mathrm{n}$ at time $\mathrm{t}, f$ is the activation function, $\gamma$ is the input scaling factor applied to the masked data Input $_{t}$ and $\eta$ is the memory or leakage parameter controlling the strength between the fixed internal neuron connections $W_{E s n}$. The equation captures the temporal and neighbouring neuron dependencies. In the case of the ring topology, $W_{E s n}$ is connection matrix that consists of a square matrix with ones in the subdiagonal.

The intention to explore combination of traditional algorithms employed at machine learning challenges, and with time not being a main concern as algorithms were allowed to run for $24 \mathrm{~h}$, influenced the choice to work on Python. The Python programming language counts on numerous ready-made libraries that facilitate the application of machine learning algorithms, as it is the case of scikit-learn [Pedregosa et al., 2011]. Moreover, the language was favoured by the organisation, as tools for facilitating code submission and scoring were made available in that language. Within the Python ecosystem, a choice was made on implementing the ESN ad hoc, as existing libraries showed interesting (weight strength) spectral radius control features but did not offer the ability to easily fine tune topology to simpler structures as those we were interested in. A version of the ESN tools developed can be seen on [Alfaras et al., 2019b].

With all the ingredients in place, data was divided into 10 -folds for crossvalidation, i.e. leaving around $10 \%$ of the test data out of the training procedure iteratively, making sure that stratification guaranteed that no undesirable class biases existed between folds. A subset of 5000 patients out of 40000 was used in order to probe the dependence on the number of neurons $(\mathrm{N})$. In order to determine an optimal value of $\mathrm{N}$, a basic grid search was conducted over the input scaling and feedback strength space. The early results showed that for $N>100$ classification performance decreased considerably. Once neurons were settled at $N=100$, the cross-validation training procedure to optimise input scaling and memory began for the whole training dataset. While mean square error minim- 
isation was the criterion for optimal weights in the solution of the linear system, parameter optimisation was guided by the area under the curve of the receiver operating characteristic (AUC). A 10-fold stratified cross-validation procedure was used, i.e. testing on folds with $10 \%$ of the training data with a similar septic-healthy proportion. Moreover, a check-up was implemented to ensure no patients used in the same iteration ever belonged to training and testing sets. Finally, with resulting binary classifications that overlapped significantly, a threshold maximising F1score is set for every parameter combination. The F1-score denotes classification performance and prioritises both precision and sensibility. The training results yielded a sepsis classifier that best performs $(A U C=0.744)$ under the following regime: $N=100, \gamma \leq 0.001, \eta \in[0.1 ; 2.5]$ (see fig. 3.9).

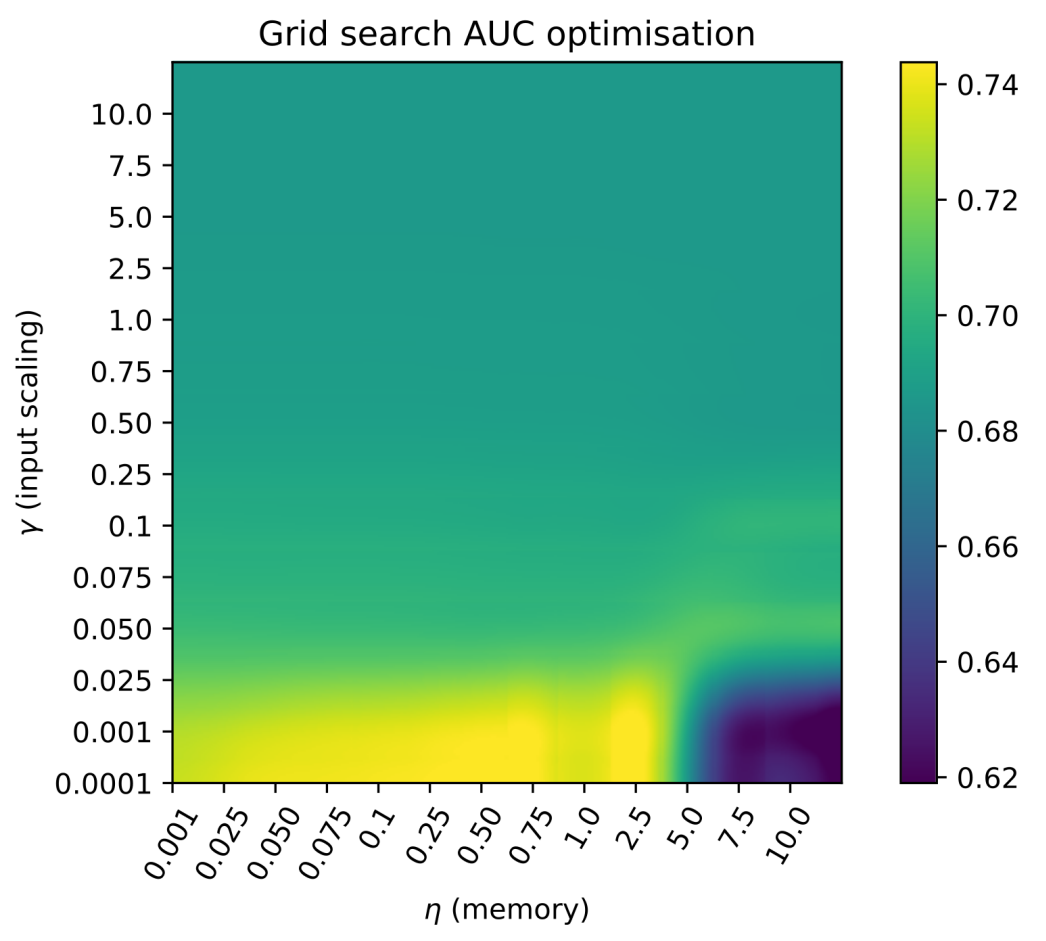

Figure 3.9: ESN AUC-based input $(\gamma)$ and feedback $(\eta)$ strength parameter grid search for the classification of septic entries in ICU medical record data.

In an attempt to compensate for the unexplored randomly chosen constant input weights at the nonlinear mapping [Rodan and Tiňo, 2011], and working with an approach closer to that of [Ortín et al., 2019, Alfaras et al., 2019a], a slight improvement of 2-3\% on AUC was verified for ESN ensembles consisting of 10 
networks or less. As compared with ECG classification approaches with echo state networks, the results of the combination fell short on classification improvements. One of the side goals of using the ESN for the sepsis challenge, was to probe and compare performances among traditionally used machine learning algorithms, the ring ESN and a combination of both. After some tests were carried out with the same data, results indicated that gradient boosting could slightly improve the ESN classification if the classifier took the network output as input. However, it was verified that gradient boosting alone or even random forests achieved better AUC values for the task at hand.

\subsubsection{Discussion: Predicting sepsis}

All in all, the implemented ESN classifier seemed to miss the health record dynamics that could shed light on the sepsis prediction problem. Apparently, the network dynamics alone were not able to separate classes significantly. Among all the entries that competed officially, no entry was free from overfitting problems. The organisation, carefully planning the challenge, sequestered data from a hospital, which remained unseen [Reyna et al., 2020]. Although high AUC or Utility scores correlated well between the two training hospital datasets, this was not the case for the unseen hospital. Moreover, clinical utility and AUC scores did not necessarily present high values simultaneously either, showing how far still algorithm performance in ML competitions is from clinical impact. In fact, instances of the competition opted to optimise for the utility function only, sometimes even coding loss functions that rated the most utility-beneficial feature sets. One of the major challenges that the ESN implementation did not solve was the great effect that the underrepresented septic class had on the training. In the competition, gradient boosting based approaches performed well, mainly those which often included features that somehow encoded the information missingness more explicitly [Singh et al., 2019, Chang et al., 2019, Du et al., 2019]. This raises concerns. If one looks at artificial intelligence as a technology that is called to support doctor expertise, the data used for the given challenge, and in particular the data missingness has to be revisited with care. In the clinical history of the subjects whose data are made available, some patterns emerge for given variables. If a subject who is being hospitalised in an intensive care unit exhibits signs of further health complications it is likely that doctors in charge ask for lab tests. In 
lab variables lies an interesting insight. As opposed to routinely collected variables such as temperature, heart rate, etc., indicators such as $\mathrm{pH}$ or lactic acid, among others, cannot be disentangled from their analytical nature. Values are present when doctors suspect problems are bound to appear. An algorithm may certainly pick on information missingness. However, by no means a classifier trained as such would bear the doctor's intelligence or intuition, likely failing to generalise well. Although not trying to argue against or in favour of automatised approaches that leave out "human-in-the-loop" use cases, it is needless to say that the question of exploiting information missingness triggered by health professional's decisions raises questions of fairness. On a separate issue, data imbalance and imputation strategies gathered some attention during the competition. Although imputation strategies did not seem to have a relevant impact, preprocessing steps considering time widow environments of the order of $6 \mathrm{~h}$ where differences of sequential values were explicitly taken as inputs made a difference. These windows, sometimes used at the time of imputation, likely contributed to enhance the impact that the low number of septic cases had on the algorithm training, either explicitly - as an oversampling strategy - or implicitly. Interestingly enough, there is a fact probably contributing to the idea that machine learning competitions are very much enriched when attracting cross-disciplinary teams that do not merely apply signal processing recipes that have become off-the-shelf thanks to widespread programming libraries. The challenge winners presented a mathematical implementation of multidimensional integral paths that to some extent encode different relative covariances [Morrill et al., 2019]. The resulting integrals are said to be a signature encoding patients' history, even said to exhibit bijective properties.

\subsection{Beyond classification: The path to real-time pro- cessing and new metrics}

We have so far seen how simplistic machine learning algorithms processing health records and biodata help making biosignals available, e.g. for the classification in health problems targeting certain conditions or characteristics. Another way to make biosignals available is to work on new features or descriptors that better characterise or unveil a condition. In this section attention is paid to other ways of making biosignals available beyond the development of simplistic ML processing algorithms, by implementing real-time processing trying to capture 
specific events or developing new descriptors combining features. The first is an example of real-time processing implementations, of relevance in health domains and for wearables. Additionally, progress can be made via exploiting biosignals in the context of human performance. In such study, electromyography and heart rate variability data are combined in order to capture the emergence of fatigue.

\subsubsection{Monitoring physiology in real-time: HRV}

In the current section, the real-time monitoring of heart rate variability (HRV) is addressed. This work led to the development of a plugin module for a biosignal acquisition and processing software platform (see fig. 3.11), OpenSignals [PLUX S.A., 2018], the official and freeware biosignal acquisition platform of BITalino and biosignalsplux. Heart rate variability (HRV) is the variation, over time, of the period between consecutive heartbeats [Acharya et al., 2006]. This phenomenon, predominantly dependent on the extrinsic regulation of the heart rate, is thought to reflect the heart's ability to adapt to changing circumstances by quickly detecting and responding to unpredictable stimuli. Its study is often carried out via the observation of RR peak interval time series or, conversely, changes in instantaneous heart rate. By means of $\mathrm{HRV}$ analysis, researchers get access to the assessment of cardiac health and the performance of the regulatory autonomic nervous system (ANS). In the work of [ChuDuc et al., 2013], a list of medical conditions affected by HRV is provided. "Heart rate variation techniques and indexes have proved crucial in treating not just cardiovascular system diseases, but unrelated conditions such as stroke, Alzheimer, renal failure, leukemia, epilepsy, chronic migraines, and obstructive sleep apnoea. Most doctors tend to agree that all biological systems, even the healthy ones tend to show haphazard dynamics while biological systems suffering from disease show reduced levels of dynamics. [...] autonomic dysfunction is a key characteristic of heart and diseases such as sepsis, brain trauma, multiple organ failure, and myocardial infarction". Moreover, when it comes to affective health, ANS dysfunction has proved to be a marker of depression. In [Beauchaine and Thayer, 2015], it is highlighted that abnormally low values and relevant decreases in the high-frequency components of HRV in resting conditions when, e.g. evoking emotions, are linked to a significant range of psychopathological syndromes (phobias, anxiety, attention problems, depression, etc.). In fact, approaches (e.g. [Can et al., 2019]) implemented for the detection 
of stress indicators often rely on the use of HRV - perhaps in line with common processing of physiology carried out in affective computing. An early work by [Zucker et al., 2009] showed how the effects of a biofeedback intervention based on the phenomenon of respiratory sinus arrhythmia, i.e. the changes in heart rate due to the thoracic cavity changes that synchronously accompany breathing, could be analysed by means of HRV and the resulting changes in post traumatic stress disorder symptoms that were targeted. Moreover, the meta-analysis [Kemp et al., 2010], for instance, concludes that correlations exist between low HRV and depression as well as acknowledging the negative correlation between HRV and depression severity.

\section{Analysing heart rate variability}

The study of heart rate variability begins with the electrocardiogram. Any ECG signal is characterised by particular patterns (heartbeats) that repeat over time. A heartbeat appears in an ECG as a specific shape or wave pattern that presents well-known peaks.

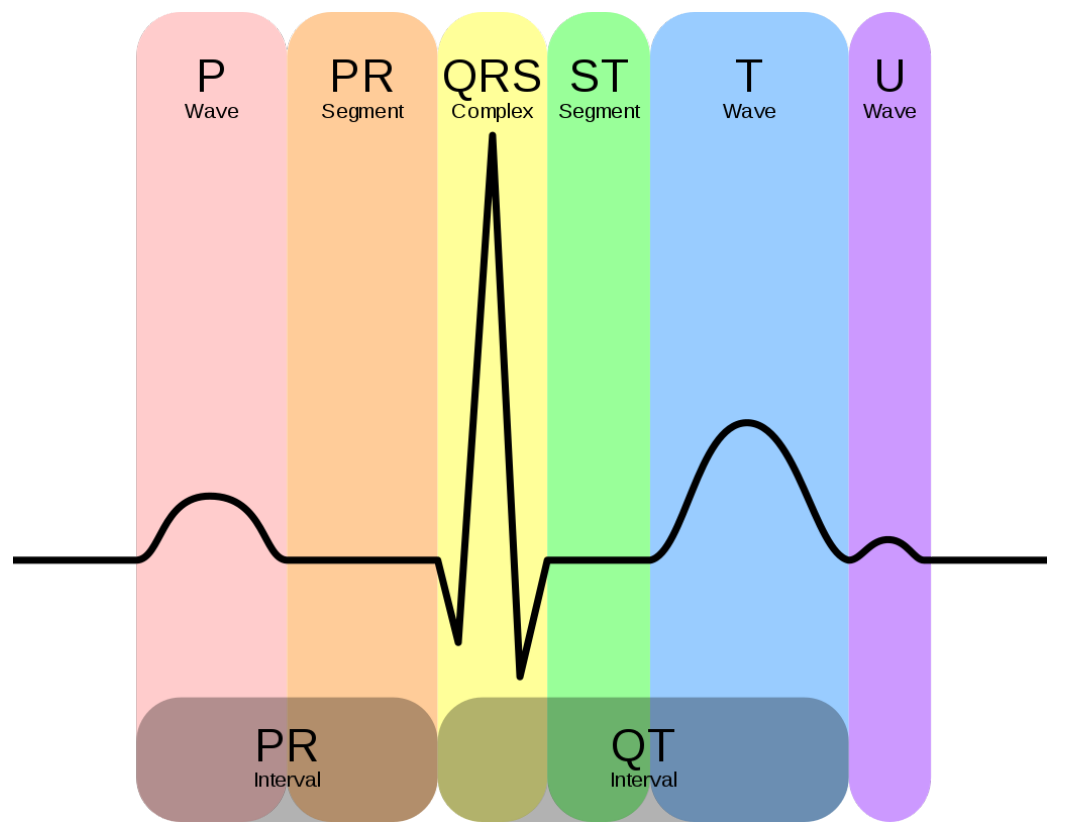

Figure 3.10: Example of an electrocardiography ECG signal and its waves and peaks [Rehua (derivative) et al., 2014]

These peaks, corresponding to the different electric depolarisation-repolarisation 
cycles that the heart muscle undergoes, mainly consist of the P-wave, the QRS complex, the T-wave and the segments in between these relevant points. The QRS complex, i.e. the most pronounced dynamics within the heartbeat, is often used to locate the different beats in an ECG signal. The significant ventricular contraction that the QRS complex encompasses, renders its most relevant feature: the high amplitude change in the R peak. ECG processing algorithms have traditionally relied on differential approaches to properly locate the $\mathrm{R}$ peaks (and heartbeats, consequently). Our idea is that of implementing the different HRV measurements using a sliding window strategy which provides time series of features that update as ECG information is being acquired. Once $R$ peaks are identified, it is possible to represent the series of $\mathrm{R}-\mathrm{R}$ intervals. This series is known as the tachogram, an it is at the core of HRV analysis. HRV features can be categorised in different groups: Temporal features (or statistics of the tachogram), Nonlinear features, Frequency features (spectral or Fourier-like) and Geometrical.

\section{List of implemented HRV features}

* Temporal features:

- $\operatorname{minRR}(\mathrm{s})$ : Minimum RR interval duration.

- $\operatorname{maxRR}(\mathrm{s})$ : Maximum RR interval duration.

- avgRR or $\langle\mathbf{R R}\rangle(\mathrm{s})$ : Average RR interval duration.

- SDNN or SDRR (s): Standard Deviation of RR (normal to normal NN) intervals.

- rmsSD (s): Root mean square of the successive tachDiff differences - an approximation to the dispersion on the tachogram's derivative, formulated as:

$$
r m s S D=\sqrt{\frac{\sum \operatorname{tachDiff}_{i}^{2}}{K}}
$$

where $\mathrm{K}$ is the number of samples.

- NN20: Number of RR intervals between non-ectopic (normal) beats with duration greater than $20 \mathrm{~ms}$ 
- pNN20: Ratio between NN20 and the total number of RR intervals on the processing window.

- NN50: Number of RR intervals between non-ectopic (normal) beats with duration greater than $50 \mathrm{~ms}$

- avgIHR or $\langle\mathbf{I H R}\rangle$ (bpm): Average of the instantaneous heart rate, computed by means of RR interval inversion.

- STD IHR or SD IHR (bpm): Standard deviation of the instantaneous heart rate.

Nonlinear features:

- SD1 (s): Major subaxis on the nonlinear Poincaré plot ellipse $R R_{i+1}=$ $R R_{i+1}\left(R R_{i}\right)$

$$
S D 1=\sqrt{\frac{S D S D^{2}}{2}}
$$

- SD2 (s): Minor subaxis on the nonlinear Poincaré plot ellipse $R R_{i+1}=$ $R R_{i+1}\left(R R_{i}\right)$

$$
S D 2=\sqrt{2 S D N N^{2}-S D 1^{2}}
$$

- SD1/SD2: Ellipse subaxis rate on the nonlinear Poincaré plot $R R_{i+1}=$ $R R_{i+1}\left(R R_{i}\right)$

Frequency features:

- HF: spectral power of the high frequency band $(f \in[0.15,0.40] \mathrm{Hz})$

- LF: spectral power of the low frequency band $(f \in[0.04,0.15] \mathrm{Hz})$

- VLF: spectral power of the very low frequency band $(f \in[0.003,0.04] \mathrm{Hz})$

- ULF: spectral power of the ultra low frequency band $(f<0.03 \mathrm{~Hz})$

Geometrical features:

- tIndex: Triangular index.

$$
\text { tIndex }=\frac{\mathrm{RR} \text { intervals }}{\mathrm{RR} \text { intervals in the dominant histogram bin } t s}
$$


In the reported work addressing real-time heart rate variability analysis, at the time a work in progress [Ramos et al., 2018], there was the aim to test which typical offline measurements were translatable to an online processing mode working with iterative windows of segmented data. Although the first implementations of online HRV assessment counted on simplified adaptive thresholds for QRS detection, the differences in generated tachogram $R R$ series made us eventually opt for the wellknown Pan-Tompkins algorithm [Pan and Tompkins, 1985], applied sequentially in the case of online processing. The Pan-Tompkins algorithm, consists in the following steps:

1. Acquire an ECG signal

2. Filter by means of a Low-pass + High-pass filter (Band-pass)

3. Derivative computation

4. Squaring step

5. Window Integrate

The application of a band-pass filter, a second-order Butterworth with frequency band-width $f_{B W} \in[5,15 \mathrm{~Hz}]$, reduces noise related to sources such as muscle activity and powerline interference. For the derivative step, sample difference approximations are used. The idea behind it is that information is obtained on the slope or trend of the changes. Finally, the resulting signal is squared to further augment the QRS complex contributions as compared to the lower peaks in ECG patterns and then slightly smoothed out via an average window. The resulting values are the integrated signal, providing the peak candidates to be compared with a threshold. Our implementation never worked on delays shorter than $8 \%$ sample delays, as that is a condition met in the original work of Pan-Tompkins (the most demanding processing step applying a delay of 16 samples at a $200 \mathrm{~Hz}$ sampling rate). Maxima are searched for in the integrated signal. We proceed with the definition of a adaptive thresholds (eq. (3.14)) that iteratively take into account the information of the previous QRS complexes detected, the current peak being evaluated and the changing levels of signal and noise.

$$
\begin{aligned}
\text { threshold } & =\text { noise }+0.25(\text { signal }- \text { noise }) \\
\text { signal } & =0.125 P+0.875 \text { signal } \\
\text { noise } & =0.125 P+0.875 \text { noise }
\end{aligned}
$$


When a QRS peak is detected in the integrated signal, the same check is carried out in the filtered signal, with independent threshold and signal/noise levels as criteria to be met and iteratively updated following the same rules (eq. (3.14)). Moreover, a rule is incorporated to discard the possibility of having two peaks separated by intervals below $200 \mathrm{~ms}$, a physiological limitation. Details on the user interface can be seen in the following image (fig. 3.11), showing a configuration pane, active features, and generated graphs:
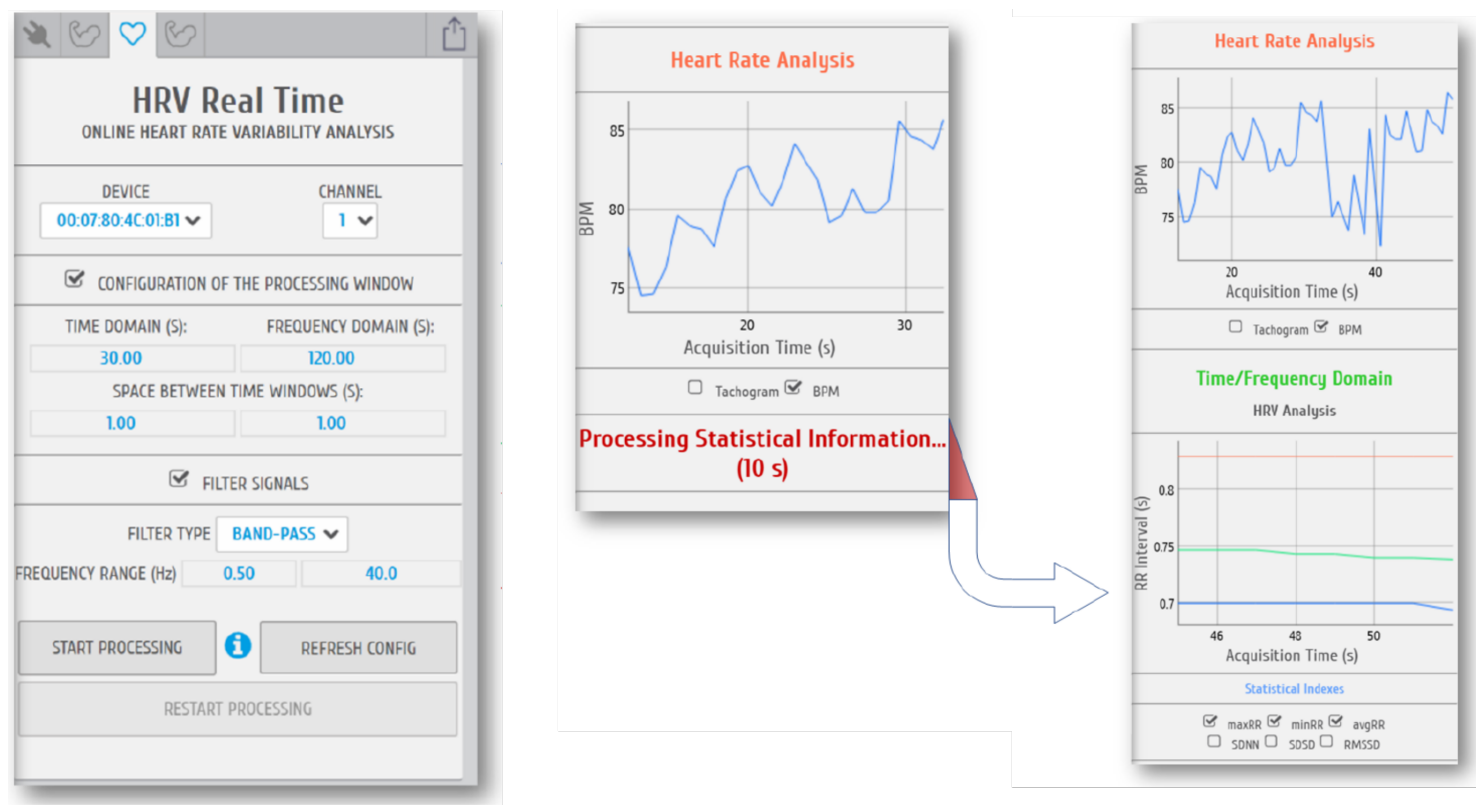

Figure 3.11: PLUX OpenSignals software HRV real-time add-on panes, with filtering settings, selected features and generated graphs (left to right)

\section{Discussion and limitations}

Although the resulting platform presented at [Ramos et al., 2018] is relevant for the use of developers and psychophysiology researchers aiming to explore different filter ranges and ectopic heartbeat exclusion criteria, the implementation of real-time HRV processing poses several concerns related to real-time limitations. According to guidelines [Task Force of the European Society of Cardiology, 1996], warm-up periods are essential to provide relevant feature values. For frequencybased features, for instance, power spectrum values are not considered reliable unless using at least time sequences of 2 minute duration or greater. This is 
derived from the fact that when using the frequency transform methods (in our case using Lomb-Scargle implementations [VanderPlas, 2018]), shifting from the temporal domain to the frequency domain presents a temporal-frequency resolution tradeoff. It is worth mentioning some of these concerns, as seen in [Task Force of the European Society of Cardiology, 1996]. The assessment of power spectrum information of the ULF frequency band $(f<0.03 \mathrm{~Hz})$, for instance, is not conceived for short term variation analysis. The spectral contribution of the VLF band $(f \in[0.003,0.04] \mathrm{Hz}$ ) should be discarded if not obtained with time series longer than 5 minutes. Furthermore, the geometrical analysis (triangular index) is only suggested for periods above $20 \mathrm{~min}$. With the goal of making biosignals available (ECG and HRV data in this case) the outcome of this collaboration led to a useful software tool that further motivates the study of HRV in real-time, with the possibility of setting up customised heartbeat excluding criteria, addressing the contribution of different power spectral ranges, the effect of different window size and overlap for the time series of features, among others. Solutions like these, combining the configuration of processing steps while allowing the representation and deeper study of acquired biosignals through functions like data exports, zoom in/out, smoothing filters, etc. are paving the way for future biomedical engineering research, biosignal processing leveraging machine learning and the like. However, the processing platform presented leaves certain aspects understudied. That is the case of further nonlinear feature analysis as originally presented in e.g. [Acharya et al., 2006], discarding instead the breadth of this research area and limiting our scope on very few features derived from the Poincaré plots whose dependence on window lengths is not covered in our study. The established use of statistically-based assessments that draw upon temporal features has achieved a standard status for several reasons. As a rationale we can argue that any heart monitoring device targets, at the very least, the tracking of heartbeat occurrences. Even with the low-fi specifications found in commercial platforms without strong scientific validity, or the usage of different sensing mechanisms such as wrist photoplethysmography (PPG) powered to track the blood volume pulse (BVP), dedicated algorithms make it possible to compensate for the technology drawbacks and achieve a reliable detection of heartbeats. The consequence is that computing intervals, heart rate and tachograms are approaches that standardise the assessment of $\mathrm{HRV}$ and allow comparisons across a wide range of platforms. That is the success of HRV. A downside presented by this situation is that nonlinear 
features, counting in fact with entropy-based, detrended fluctuations and many other measurements beyond Poincaré-based magnitudes [Melillo et al., 2011], receive less attention. However, the significant information intrinsic to the ECG morphology - perhaps entailing a more nuanced or controlled acquisition design depending on leads, electrode placement, etc. - is discarded completely in HRV. ECG morphology, as it can be seen in section 3.3.2 and the work presented in [Ortín et al., 2019, Alfaras et al., 2019a], is able to unveil relevant processing capabilities in the domain of health. While HRV makes a great case for the creation of tools that facilitate customisation of the analysis steps, platforms called to promote biosignal understanding should embrace approaches beyond timebased processing.

\subsubsection{Combining biosignal features into new descriptors: The case of fatigue monitoring}

Fatigue is a complex psychophysiological phenomenon with relevant impact in sports performance and in the correct functioning of our motor system. Physical fatigue is an objective phenomenon that decreases the mechanical exertion capabilities. On the contrary, mental fatigue, subjective and related to cognitive stress, has an impact on psychological factors such as concentration and alertness. In the context of sports, fatigue can have a negative impact on performance or, even worse, lead to injury or health complications if prolonged in time. Past research has shown that muscle injuries are more frequently associated with prolonged forms of muscle fatigue [Rozzi et al., 1999]. To this regard, the development of a monitoring system that identifies the onset of fatigue and monitors it, incorporating mental and physical fatigue features in a non-invasive manner for the sport under study would be of relevance. In order to do so, we suggest the creation of new fatigue indicators that combine electromyography (EMG) and heart rate variability (HRV) features derived from electrocardiography (ECG). Our study [Ramos et al., 2020] builds upon physiological data acquired during cycloergometry exercises.

\section{Assessing fatigue: Tests and features}

Our research of fatigue onsets and indices draws on conducted cycloergometry tests and physiological monitoring. With university institutional review board approval and having provided signed informed consent, the study participants were 
recruited from a local university community through invitation and flyers. The group of participants consisted of fourteen healthy subjects (men, aged $24.5 \pm 3.6$, body mass index $=23.7 \pm 1.7 \mathrm{~kg} / \mathrm{m}^{2}$ ), free from known cardiovascular/metabolic disease and medication intake, nonsmokers and normotensive i.e. with consistently bounded blood pressure values $(80$ and $120 \mathrm{mmHg}$ reference upper values for sistolic and diastolic). Before testing, participants were requested to avoid heavy physical activity for $24 \mathrm{~h}$, caffeine drinks, and food intake from midnight to the morning testing session. The monitoring sessions were scheduled twice in a 7-day period for each subject. The two sessions were held at similar times (between 7a.m. and 10 a.m.) with no more than $48 \mathrm{~h}$ between them. On day 1, subjects' weight and height were measured, alongside a "Graded Exercise Test" providing a treadmill-based estimation of their peak oxygen uptake $\left(\mathrm{VO}_{2}\right.$ peak $)$ and second ventilatory threshold $\left(V T_{2}\right)$. On day 2, subjects underwent a "Constant Work Test" on the cycloergometer working at the established constant work rate $\left(W R_{V T_{2+15 \%}}\right)$, with constant cycling cadence and synchronising their right leg with a metronome speaker. EMG signals from 2 of the muscles captured during exercise (rectus femoris and vastus medialis) and heart rate variability data were used for our analysis. The HRV records of 3 participants were excluded, as they suffered from data loss issues.

\begin{tabular}{lll|ll}
\multicolumn{2}{c|}{ EMG } & \multicolumn{2}{c}{ HRV } \\
\hline Time & Frequency & Time-frequency & Time & Frequency \\
\hline rms & Median freq. & Median freq. & Max RR & ULF power \\
& Total power & Major freq. & Min RR & VLF power \\
& & Major time & Average $\langle R R\rangle$ & LF power \\
& & Mean power & SDNN & HF power \\
& Area & rmsSD & Median freq. \\
& Volume & Triangular I & \\
& Time dispersion & SD1 & \\
& Freq. dispersion & SD2 & \\
& & SD1/SD2 & \\
& &
\end{tabular}

Table 3.1: EMG and HRV parameters for the study of fatigue

Data was recorded during cycloergometry exercise (acquisition+ phase) and as during post-exercise recovery (acquisition-phase). Of particular interest are 
the rectus femoris acquisition+ signals, i.e. a muscle that is heavily affected by the buildup of fatigue and the vastus medialis acquisition- signals, a muscle relatively less or not affected by fatigue during the test. Applying the Teager-Kaiser Energy Operator on the EMG signals, muscle activation events were monitored. As per HRV data, R peaks were computed following the Pan and Tompkins procedure, yielding the RR intervals for the tachogram time series representation. Using an overlapping sliding window approach, with different sizes, several time series of EMG and HRV features were created to represent their evolution (see table 3.1). The time-frequency analysis of the EMG, circumventing the nonstationarity problems of EMG Fourier transforms, is derived from the use of the wavelet transform with the $\Psi$ Morlet prototype wavelet:

$$
\Psi_{\text {Morlet }}(t)=\frac{1}{\sqrt{b \pi}} \mathrm{e}^{-\frac{t^{2}}{b^{2}}} \mathrm{e}^{j 2 \pi f_{c} t}
$$

In the wavelet function of time (t), $j$ is the imaginary unit in the sinusoidal term $\mathrm{e}^{j 2 \pi f_{c} t}, f_{c}$ represents the central frequency, and $b$ is the bandwidth parameter playing the role of a Gaussian window width related to the energy spread in the frequency domain. The time-frequency domain analysis is carried out by iteratively scaling the wavelet and obtaining the scalogram $S(t, f)$ based on a given set of pseudofrequencies, yielding the features (Major time, Major frequency, etc.). The resolutions used were $\Delta t=0.001 \mathrm{~s}$ and $\Delta f=2 \mathrm{~Hz}$. For each subject Subject $_{i}$, parameters $P_{k}$ are extracted using a wide range of window sizes $W S^{E M G}$ and $W S^{H R V}$, in turn evaluated for different time steps $T S$ :

$$
\text { Subject }_{i}\left\{\begin{array}{l}
\text { For each parameter } P_{k}^{E M G} \text { and } P_{k}^{H R V} \text {, respectively } \\
\text { Compute series with } W S^{E M G}=[5,10,15,20,25] \text { muscle activations } \\
\text { Compute series with } W S^{H R V}=[30,40,50,60,70,80,90,100,110,120] \mathrm{s} \\
\text { Repeat for different time steps } T S=[0,10,25,50,75,90] \% \text { of } W S
\end{array}\right.
$$

For EMG parameters, the application of the different window sizes means that features are computed per activation and then averaged to obtain a single measurement. The time series were obtained for all the parameter, window size and time step combination, for the $14 \mathrm{EMG}$ and $11 \mathrm{HRV}$ samples, respectively. A trend was derived from every time series by means of fitting a linear regression model to the values. This yielded slopes $(m)$ and standard deviations $(\sigma)$ for every parameter, which was initially used to characterise the trend as a growing or descreasing 
one. More research should be done in order to justify the procedure, as despite the usefulness in helping reduce the complexity of the problem, concerns emerge regarding the vague linearity assumption on several of the parameters. Looking at the whole population of subjects, it was possible to combine parameter trends and obtain a general average trend for each parameter at a given window size and time step:

$$
m_{\text {comb }}=\frac{\sum^{N} m_{i} w_{i}}{\sum w_{i}} \quad \sigma_{\text {comb }}^{2}=\frac{1}{\sum^{N} \sigma_{m_{i}}^{-2}}=\frac{1}{\sum^{N} w_{i}}
$$

For every pair of $W S$ and $T S$, a coefficient of variation $C V$ is computed (eq. 3.17) in order to characterise which combinations render the most relevant variation (high $m_{\text {comb }}$ ) with the least uncertainty (low $\sigma_{c o m b}$ ), i.e. presenting a low $C V$ :

$$
C V(W S, T S)=\frac{\sigma_{c o m b}}{m_{c o m b}}
$$

In order to rule out single-subject effects and focus on fatigue-induced states, the trend analysis is restricted to the $W S-T S$ combination that minimises $C V$. Moreover, two conditions had to be met. A trend for parameter $P_{k}$ is not considered as such, when its acquisition+ value $\pm 95 \%$ confidence interval crosses $0-$ i.e. implying a change of sign or trend reversal. Any acquisition+ trend must also verify that its acquisition- counterpart presents a trend reversal or certain degree of uncertainty that would allow it. When both criteria hold, a parameter $P_{k}$ is said to be an individual fatigue descriptor (IFD). The resulting features (4 EMG and $10 \mathrm{HRV}$ ) that are left are Fourier median frequency, Wavelet median frequency, Wavelet major frequency and Wavelet major time for EMG, and Max RR, Min RR, $\langle R R\rangle$, rmsSD, SDNN, Triangular index, LF power, HF power and Fourier median frequency of the tachogram for HRV data.

\section{Combining fatigue descriptors into a global index}

In order to further characterise fatigue, our study gathered all the individual fatigue descriptors (IFD) identified and combined them into what we called a global fatigue descriptor (GFD). Using the inverse of CV as a weight - hence, prioritising 
the contribution of IFDs with low CV - a weighted average is computed as follows:

$$
G F D[i]=\frac{1}{C V_{k}} \frac{\sum^{K} I F D_{k}[i]}{\sum^{K} C V_{k}^{-1}}
$$

The IFDs used, are previously normalised, using the parameters' maximum values obtained in the cycloergometer test. The resulting GFD, with values comprised between 0 and 1, can be analysed along time for each participant, capturing a fatigue profile for each one of them. An example is shown below. Using statistical quartiles segments are established in the GFD values in order to characterise what we propose as dangerous fatigue. Red zones, delimited by the first quartile, highlight dangerous levels of fatigue. Green zones (above median) denote non-dangerous levels of fatigue. The area in between is a transitional regime:

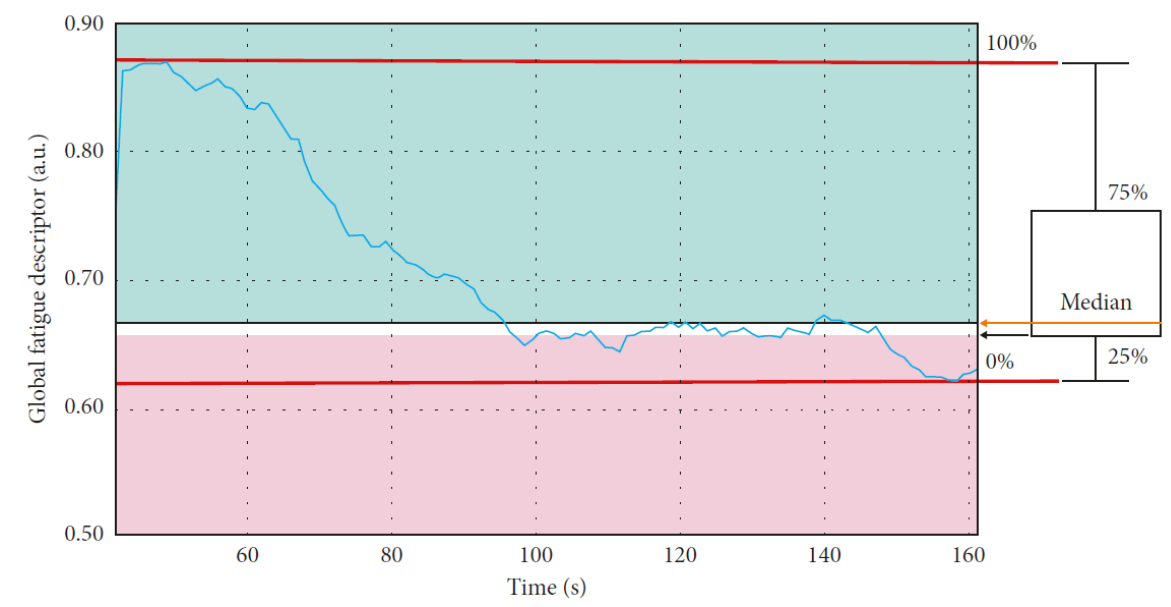

Figure 3.12: Example of a Global Fatigue Descriptor (GFD) and its dynamics for a given subject. Red (below first quartile) and green (above median) ranges denote dangerous and non-dangerous fatigue levels, respectively.

Empirically, it is observed in the collected data that, in general terms, GFDs present an abrupt declined followed by a prolonged stability period. The observed behaviour, can be interpreted based on the physiological mechanisms taking place during fatigue buildup. When exercise starts, energy production is led by efficient and slower aerobic breathing. When the maximum rate of aerobic energy production is reached, a faster anaerobic respiration gradually takes over to fulfil 
the energy needs. The aerobic-anaerobic transition, starting which what is known as the first lactate threshold could be linked to the emergence of the "stability" GFD period [Faude et al., 2009]. In order to classify the fatigue regime, we choose to train a support vector machine (SVM), an algorithm that learns what hyperplane best separates data classes while maximising margins (distance to the datapoints of the different classes). EMG and HRV time series of the 11 subjects are split in two segments of the same duration. Two features are computed, encoding the average value and the variation rate of the normalised IFD parameter series:

$$
\text { feat }_{1}=\frac{\sum^{L} P_{k}[i] / \max _{P_{k}}}{L} \quad \text { feat }_{2}=\frac{\left[P_{k}^{\text {end }} / P_{k}^{\text {start }}\right]-1}{t_{\text {end }}-t_{\text {start }}}
$$

$P_{k}[i]$ is the parameter value at every given sample $\mathrm{i}, L$ is the number of samples in the time series segment, $P_{k}^{\text {start }}$ and $P_{k}^{\text {end }}$ represent parameter value at the start and finish time instants $t_{s t a r t}, t_{e n d}$, and $\max _{P_{k}}$ is the calibration maximum for parameter $P_{k}$.

Hence, any pair of EMG and HRV series was encoded in $2\left(N_{E M G}+N_{H R V}\right)=$ 28 values. Using a leave-one-out cross-validation strategy, a recursive feature elimination taking care of redundant information yielded feat $_{1}$ for the Wavelet median freq., Wavelet major freq. (EMG), $\langle R R\rangle$, SDNN, Triangular Index, SD2, Fourier median freq., LF power and HF power (HRV). In total, 9 features are left.

Since we are interested in assessing fatigue classification in real time, the SVM testing phase disregards averages and feeds instantaneous values to the classifier. The classifier performance was rated by means of a stratified 11-Fold crossvalidation procedure, obtaining an accuracy of $A C C_{S V M}=0.82 \pm 0.24$. Example classification outputs can be seen in fig. 3.13.

\section{Discussion and limitations}

Our approach shows how by means of a broad analysis of EMG and HRV parameters and their trends, combined with normalisation values obtained on cycloergometry exercises, it is possible to find candidate features (IFDs) that describe the buildup of fatigue in general. The idea behind this characterisation is very appealing, as it could made biosignals available for the real-time monitoring of fatigue that could prevent injury or reduced muscle performance long before any damage takes place. However, our study poses several limitations besides 


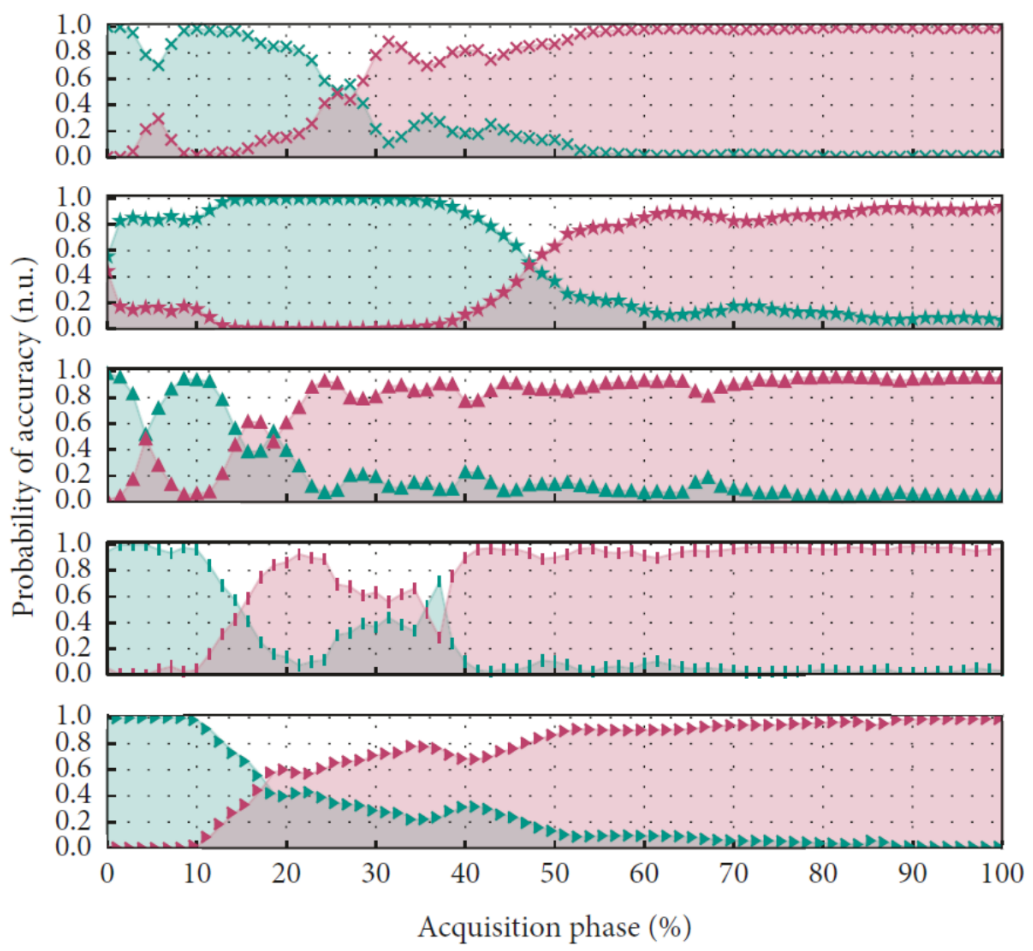

Figure 3.13: Support Vector Machine (SVM) fatigue classifier output for 5 subjects. Green datapoints represent non-fatigued class outputs and red datapoints represent fatigued class outputs. 
the small population it is based on. By means of a weighted average that favours relevant change in values and low uncertainty IFDs are then combined into a global fatigue descriptor. Although the idea of a GFD index that characterises the fatigue state is very powerful, as well as their graphic representation, the optimisation of window sizes and time steps renders IFDs that are computed with under different conditions - a process that does not likely generalise well. Fatigue regimes in the GFD and their boundaries are merely defined based on statistical quartiles. Without established window sizes and time steps that work in general, the approach proposed is still costly in terms of computational power. Experimentally, how dependent fatigue characterisation is on the chosen muscles has not been studied. Without this validation, there is no guarantee that fatigue buildup generalises to other muscles. Calibration, or the obtention of normalisation values limits the broader applicability of the characterisation we describe. Moreover, although it is empirically observed that the GFD generally follow a decrease trend, values for different subjects do not necessarily conform to fatigue regimes (ranges) determined during calibration and exceptions to the decreasing dynamics exist. While the SVM classification in line with real-time results work relatively well at depicting the transition from non-fatigued to fatigued regime, a few subjects' representation of output classes over time pose concerns. These emerge either because fatigue is never established or because non-negligible regions of nonfatigue states appear after fatigue has settled in - an unexplained behaviour present in about half of the sample subjects. Finally, the application of a simple Principle Component Analysis (PCA), a dimensionality reduction method that removes redundancies, shows that the 9 features used in the classification could be reduced to 2 principal components. 
PART II 



\section{Chapter 4}

\section{Biosensing, body self-awareness and first-person design}

\subsection{Making biosignals available: The body, interaction design and affective technologies}

In recent years, as personal sensing devices have gained popularity throughout mobile phones, applications that go beyond behavioural health and sport selftracking wearables - such as FitBit, AppleWatch or SamsungGear implementing heart-rate monitors -, interaction design research has started to call for alternatives to challenge, rewrite and appropriate meaning-making around biosensors and the signals from our body. Biosignals are powerful. They stem from the medical practice and capture body and physiological insights to expose them. In combination with the growth of biomedical wearables, biosignals have spurred research directions on the anytime/anywhere monitoring of the body that could have not been anticipated (see the review [Dunn et al., 2018]). Being able to take biosignals out of the lab, far from clinical settings, opens many possibilities. Especially when telling us about our emotions, our health or even performance, biosignals create a close tech-user rapport where authority and meaning are not easy to challenge. Outputs from sensing platforms backed up by science, with measuring mechanisms counting on different degrees of transparency, are subject to technology preconceptions. Sensing outputs do not only unveil rich 
body features the user might not be aware of but inevitably deliver information with certain authority, preattached meanings and health connotations that are not easy to circumvent. By authority, we refer to scientific/medical rigour and certainty that biosignals inherit from the clinical practice. When interacting with biosignals, in the form of personal sensors or trackers, the information delivered by these platforms carry such meanings. Fear and respect are common elements of that interaction. Biosignals carry meaning on aliveness, sometimes on performance or correspondence to standards [Howell et al., 2019, Merrill et al., 2019]. In part it is the interaction designer or engineer's responsibility to add the context to expose or allow that interpretation flexibility if desired, playing with ambiguity if needed [Sanches et al., 2019a].

\subsubsection{The problem}

In this research stage, the problem that is addressed, i.e. "Making biosignals available: The body, interaction design and affective technologies", consists in the following: In order for biosignals to be available for the design of affective technology and body/emotion self-awareness experiences, biodata must be understood and exposed differently. We need to approach the designer's practice, using the engineering perspective's takeaways but going beyond. As a way to challenge what is possible, a collection of interaction design principles are taken from human-computer interaction and design research.

Biosignals, as traditionally used in clinical contexts, have meanings attached, certain scientific authority, and predominant ways to be understood and presented. Biodata, in itself, has been used only to some extent, and the most predominant examples in technology-based interactions fall into a performance, self-improvement, goal-oriented paradigm (notably in physical activity). In the case of emotional sensing or tracking, that is no exception. Either through biosensing or behavioural reporting, emotion tracking examples mostly present this improvement or efficiency view of ourselves. When it comes to the presentation of biodata, i.e. the way the user is provided with (bio)feedback information, screen-based visual charts inherited from the clinical/electrophysiology practice are the norm. Alternative ways to present biodata, features or cues are sought. Hence, the key problem is posed: 


\section{How can biosignals be made available for the design of affective technology and body/emotion self-awareness experiences?}

That poses the question of how to engage bodily with biodata related to the body and emotions. How can biodata present material affordances? Or, in other words, how can one transform biosignals into something a designer can give form to, adapt, repurpose, stretch or modify as he/she pleases? If we consider biodata as a material, one can argue that biodata is not a malleable material to design with. This, of course, differs substantially from designing artefacts or experiences with tangible materials found in crafting practices, such as using wood, fabrics or metals. By affordances, what is meant is the properties of the materials that show or indicate users what can be made with them, tacitly. As introduced in section 1.1.1, the biosignals acquired by electrophysiology sensors are a computational material, often based on fluctuations of body properties that are digitised. Research on the ways in which biodata can be stretched, adapted, modified and interpreted is needed. But this is a major challenge, as biodata is not tangible, rather immaterial, and difficult to become attuned to. That is why relying on the inspiration given by interaction design research that advocates the material properties and challenges or redefines the immateriality of interaction elements resonates with our goals. On another front, the field of Affective Computing, i.e. computing that relates to, arises from, or deliberately influences emotions, needs consideration. Its development, for more than two decades, and many of its advances, draw on the recognition, detection and classification of traits or emotions. This paradigm has understandably evolved hand in hand with machine learning, feeding back in algorithm refinement and establishing vast databases to continue improving. However, that is not the full spectrum of the Affective Computing field. If a more important role for the body in the interactions is what is sought, design methods that argue in favour of doing so come in handy and provide rigour and solid foundations. This is, understanding the interaction body not only as something to be measured but as the core to feel, perceive, reflect and interpret.

In this part of the thesis, the core part of this $\mathrm{PhD}$ research, I present how interaction design is used to help researchers probe assumptions on the construction of meaning around the body and biodata. In particular, biodata generated by sensing technologies (see section 2.1.1 and 2.1.2) is of interest here. In challenging the role that personal sensing plays in technology-mediated interactions, we turn to 
the body and the design of interactions that touch upon movement, affect -as interaction-, emotion and sharing that lets us see beyond. This part of the thesis is structured under the following sections:

- Materials and methods utilised within the explorative research presented, and which proved to be inspirational for the studies carried out, are introduced:

1. $\mathrm{HCl}$ waves and the third-wave context (section 4.2.1)

2. First-person perspective design (section 4.2.2)

3. Soma design (section 4.2.3)

4. An exploration of Haptics and the body (section 4.2.4)

- Affective technology. Interacting with the body (section 4.3) provides research context and describes the way affective technology is addressed through the conducted studies. The described affective technology context and the first-person/soma design perspectives introduced set the ground for the first set of $\mathrm{HCl}$ research studies:

1. The ThermoPixels kit (section 4.4)

2. Designing (with discomfort) from a first-person perspective (section 4.5)

3. Dare to design: Sensory misalignment (section 4.6)

- Inspired by the first-person and soma design perspectives, a First-person Physiology approach takes form (section 4.7). Research is conducted to investigate how biosignals can be explored somaesthetically, integrated in biosignal acquisition and processing efforts that revert to the following study and subsequent topics that precede the final research outlook:

- From Biodata to Somadata (section 4.8)

Making biosignals available for interaction design offers interesting possibilities. With regard to affect, as it will be shown, making biosignals available is of relevance. In the particular context of affective technology design, interaction design research on arousal is used to probe the creation of representations of biodata that accompany users in the understanding and redefinition of meanings, scenarios and ownership surrounding biosignal-based emotion technology (see section 4.4, [Umair et al., 2020]). Guided by a first-person design approach (section 4.2.2), 
with tools that let the design researcher highlight the aesthetics of her/his own experience, we try to assign novel meanings to biodata, or even uncover them, almost like turning biosensing into a malleable material that one can change, shape and interpret. Somaesthetic design [Höök, 2018] (section 4.2.3), promotes a shift towards experiential qualities of interaction and putting the focus on the subjective sentient body as the core for cognition, emotion, perception and action as opposed to the traditional Western dichotomy of body vs. mind. Inspired by soma design researchers and their body-centred practices, first-person experiences guide a process to embrace discomfort, explore sensory misalignment, and more importantly, create novel interactions that make the body central in an experience that not only measures body properties as mere inputs but connects the user to them via actuation feedback that spurs self-reflection, reinterpretation and sharing. Biosignals become a malleable element to design with (section 4.8).

\subsection{Materials and methods for the interaction designer}

In order to address how biosignals can be made available for interaction designers working with (affective) technologies, the research carried out in this thesis draws on several $\mathrm{HCl}$ avenues. The field of Human-Computer Interaction has undergone many perspective shifts (called waves) that yielded particular methods or ways of working. The work presented here, is inspired by, e.g. research fields such as Affective Computing, intersecting with cognitive sciences and motivating artificial intelligence advances. However the thesis research is best contextualised with what is known as the third wave of $\mathrm{HCl}$, which could be dubbed "embodied interactions". With these tools, an approach is taken on transcending dualism and sharing subjective perspectives through exploratory group design sessions and leveraging meaning/making processes via "learning or thinking by physically doing", most notably with a first-person perspective and Soma Design concepts. In this section, a short context is provided on the Third wave of $\mathrm{HCl}$ (section 4.2.1), first-person design (section 4.2.2) and somaesthetic design approaches followed (section 4.2.3) - with a note on an inspiring haptic-body exploration exercise (section 4.2.4) that set the ground for some of the studies undertaken in this research. 


\subsubsection{The third wave of Human-Computer Interaction}

In the 1980s, computing technology started to make it into people's lives thanks to personal computers, providing accounting aids, entertainment and organisational tools to be used at home by the layperson. This fact put the focus on how computers can be useful and understandable in the absence of advanced training, creating a whole new discipline. Human-Computer Interaction, or $\mathrm{HCl}$, is an academic and design discipline concerned with the study of how people interact with computing technologies - or, more broadly, digital technologies. The idea behind the design aspect is that it is crucial to understand how to best create computing technologies that are usable, effective, and adopted by the users, in a way, engineering the human factors of technology design. The research area has its foundations on computing, together with social disciplines such as cognitive science, psychology and/or sociology, providing the grounds to address how people make use of the technology in different contexts, how interactions lead to knowledge or how technology meanings are conveyed. On the design side, and spurred by the graphical user interfaces that motivated the vast adoption of computing, there is interest in designing effective visualisation mechanisms. Another design concern is the emergence of cooperative work supported by computers, and how technology can motivate this kind of interactions. However, and especially notable after the 2000s, as computers have made it into all sorts of technologies, ubiquitous technologies are no longer necessarily dependent on screens and are virtually found in every possible object one can imagine - more so in the internet of things paradigm where any object could potentially be connected and exchange information through a network. The impact of aspects touching usability, acceptance, enjoyment or even ergonomics comes to light. Technology design cannot be separated from user experience design. Computers, or digital technologies, have use cases, contexts and goals embedded in them. Among $\mathrm{HCl}$ scholars, it is recognised that the development of the discipline has gone through three distinct waves. These historical paths can be approached by reading the works of Harrison, Bødker, Sengers and Tatar, among others [Bødker, 2015, Harrison et al., 2007, Dourish, 2001, Blevis et al., 2014]. A summary of the $\mathrm{HCl}$ waves is briefly presented here:

1. First wave: very much related to its computing and engineering origins, the first wave of $\mathrm{HCl}$ is technical, and revolves around the concept of metrics 
and guidelines. Through these tools, the use of technology is analysed - isolated to some extent. The human is an object of study. Stemming from the concept of Human factors and ergonomics, i.e. the application of psychological and physiological principles to the engineering of products, $\mathrm{HCl}$ inherits from industrial design the productivity and safety scope. Humans interact with artefacts (or computers). $\mathrm{HCl}$ seeks to compute metrics, run structured tests and focuses on skill to obtain an optimised, productive, "man-machine coupling". In words of Duarte et al. [Duarte and Baranauskas, 2016], "interaction is perceived as a form of man-machine coupling, which can be improved by solving ergonomic issues and interaction disruptions".

2. Second wave: The second wave of $\mathrm{HCl}$ is characterised by a turn to cognitive science. The human is seen as a processing entity, to be compared with, combined and studied alongside the machine. Theories are sought or strengthened to understand what the mind thinks, being studied while unaltered by the researcher. Moreover, interaction is not merely seen as something that takes place between a machine and a person, but in specific settings. These spurred contexts of work and communities of users, highlighting cooperation, understanding, learning and participation. Through the cognitive inspiration, a set of aspects are addressed, such as the fact that information needs to be communicated efficiently, be processed in and out of the system, drawing attention to user interfaces to communicate with the machines.

3. Third wave: The third wave of $\mathrm{HCl}$, revolves around usage of technology that is not circumscribed to the workplace. Instead, (computing) artefacts are found and used at home, in our everyday lives. This puts a focus on culture, and how users actually utilise technology. From an embodied interaction perspective, thinking is no longer exclusively abstract but realised through doing things in the world, expressing through gestures and learning through manipulation [Harrison et al., 2007]. Meaning making is central to $\mathrm{HCl}$, built collaboratively and on the fly. Technology impacts society globally. Values are entailed in the design of technology and interactions. While not in search of universal laws Third-wave $\mathrm{HCl}$ is much more inclined to study the many aspects that were not addressed in previous waves, such as tangible interfaces and pervasive/ubiquitous computing. To some extent, in this wave 
the recognition of multiple perspectives and scenarios gains prominence, and in turn the plurality motivates the study of situated, local, user practices.

Some $\mathrm{HCl}$ researchers are proponents of a current fourth wave encompassing a transdisciplinary paradigm [Blevis et al., 2014], apparently leveraging an orientation toward values such as ethics, equity or social responsibility among others. While these are aspects that resonate with the work presented in this research (somewhat observed via the third wave societal impacts too), the studies undertaken throughout the exploratory $\mathrm{HCl}$ journey presented here are eminently third-wave, i.e. addressing meaning making, ubiquitous technologies, embodiment strategies and recognising material affordances.

\subsubsection{First-person perspective design}

First-person perspective design is an approach at designing interactions and technology that places the user's lived experience at the core of the design process [Höök et al., 2018]. Emerged in Human-Computer interaction research contexts, the first-person perspective tries to prioritise designing for a lived experience. In doing so, the role of the designer is in turn that of the user, as interactions and technologies are tried on, experimented and tested. Goals may entail gaining a deep and experiential understanding of the effect of technology on people, society, and everyday life [Lucero et al., 2019]. In practical terms, first-person research is research that involves data collection and experiences from the researchers themselves, as opposed to external users. Höök and co-authors argue that, nowadays, most designed systems are continuously modified whenever used by users, i.e. whenever realised in the world. The end user's behaviours have an impact on systems, ultimately altering how the meaning of a given technology or interaction is built, hence leading to a never entirely finished system. The first-person researcher draws on that statement and takes a step to actively adopt a hands-on evaluative or exploratory stance. Potentially, this perspective can open ways for engaging differently with technology. The designer steps into the user's shoes. When related to design concerning the body, the distinction between a first-person and a third-person body perspective can be traced back to the French phenomenologist Merleau-Ponty [Merleau-Ponty, 2002], who sees the lived body (first-person) as different from the body as an object (third-person). Studies on user-centred design, i.e. design research that focuses on the users' needs and 
deploys validation mechanisms to approach them, often rely on a third-person perspective involving the observation of users, tracking of their behaviours, or even establish contact with them in the form of questionnaires and interviews. While the growing interest in this approach highlights the opportunities to harness new ways to create interactions and engage early on with effects, design options, and meanings that interactions spur, the first-person design perspective also poses concerns. Although handled carefully with group design sessions involving several first-person accounts, design researchers, for instance, call for attention on the fact that this way of designing subjectively may risk the fact of not accounting for users' versatile preferences, differences in perception, body, and lived experiences even if exposed to the same interactions.

\section{Autobiographical design}

Methods employed in anthropology, such as autoethnography, are very much in line with - and to be partly responsible for - the recent adoption of first-person approaches in $\mathrm{HCl}$ and will probably continue to feed on design practices around technology and interactions. $\mathrm{HCl}$ researchers have in fact "attempted to reconcile autoethnography with a more traditional view on methods" [Lucero et al., 2019], by using a scientific prose that distances itself from evocative narratives or by drawing autoethnographic design guidelines.

\subsubsection{Soma design}

Soma design, short for somaesthetic design, is a design approach that builds on the work of Somaesthetics [Shusterman, 2012] combining the body with aesthetics. The soma design framework offers a coherent theoretical basis starting from the constitution and morphology of our human body and perception. In soma design a focus is put on the felt body [Höök, 2018]. In a design process, the body is important because it is through the body that people feel, perceive and interpret the world. Somaesthetics promote cultivating our (bodily) aesthetic appreciation. When engaging in an experience, the body can be used to elucidate what the aesthetics of the experience are. Somatic experiences seek letting designers examine and improve on connections between sensation, feeling, emotion, subjective understanding and values. In order to apprehend one's sensations and exert control over them, training is needed. $\mathrm{HCl}$ research works on the soma to 
unpack several practices that have been used to get attuned to the body and how interactions are perceived. Among others, examples of body-centred practices used in soma design research are Feldenkrais - i.e. body awareness through movement - , which consists in guided movement exercises highlighting body symmetries, positions and sensations while taking a non-judgemental perspective, yoga, contact improvisation dance (see section 4.5.1) or body scans that guide one's attention towards body parts through instructions similar to those used in meditation. The works of Tsaknaki et al. and Windlin et al. provide useful descriptions for the novice [Windlin et al., 2019, Tsaknaki et al., 2019]. Moreover, in order to reach a heightened body awareness, soma design employs techniques such as estrangement [Wilde et al., 2017] or engaging with the non-habitual, i.e. performing actions, movements and gestures in ways we are not used to. A good companion of body awareness exercises is that of body sheets or maps [Khut, 2006]. These are body sketching figures that are used to reflect on body sensations at different stages of the exercises, body practices or interaction design activities conducted by design participants or users. These are presented with body sketches that need to be personally coloured, extended, and drawn according to body sensations so that group discussion is motivated and change is stressed for the participant, as body maps show the differences in sensations "captured" at different instants. As argued by Höök and colleagues, Somaesthetics is an ethical and aesthetic project that makes us aim at "living a better life". Soma design (together with a first-person perspective) approaches ethics quite uniquely [Höök, 2020, Höök et al., 2019], and even goes beyond in questioning body politics or pre-agreed norms within a cultural context. "Technologies encourage certain movements,certain aesthetic experiences, certain practices, and responses, while discouraging others, therefore shaping our ways of being. As with any design process, when one shapes an interactive artefact, future users' bodies and movements are shaped too. And it is in that interplay of movements and adaptations of behaviors that the political is enacted and enforced" - where the political is to be understood as the cultural norms and freedoms that our bodies live in. A soma design process engages personally with the digital and physical materials, but also takes the end-users' somas into consideration. As phrased by Loke and Schiphorst [Loke and Schiphorst, 2018], through the somatic perspective, self-awareness or self-care goes beyond narcissism to empathy. 


\subsubsection{Haptics and the body}

This thesis draws on a remarkable experience that stood out for a long period. This is the exploration of haptics via what is called an Aesthetic Laboration (Alab) or Sensitising lab [Akner-Koler and Ranjbar, 2016]. Akner-Koler, who had developed this method to bodily engage with the affordances of haptics - i.e. the properties that touch and touchable elements suggest tacitly in terms of gestures, directions, and ways to manipulate -, joined the first-person training event to facilitate a design session where somaesthetic actuation technology was to be combined and explored. An A-lab is a structured method for interacting with physical and digital materials that aims at increasing one's aesthetic sensitivity. As we will see, this method will impact research directions followed in section 4.7 and section 4.8, together with the Soma Bits toolkit project [Windlin et al., 2019]. With the soma design approach, A-labs foster the ability to feel and articulate different bodily sensations and properties of materials (lightness, softness, warmth, among others). Akner-Koler's approach offers structure to soma design explorative sessions. Sorted in groups with clearly delimited roles, participants share firstperson accounts of experiencing the material affordances. In the context of an A-lab first-person design workshop organised as part of a doctoral training week, for instance, groups were made of three participants. The roles were the sentient subject, the research conductor applying the requested effects or materials and the person in charge of documenting the experience and evoked body sensations. In order to obtain a heightened haptic perception, the sentient participant is blindfolded. Actions are continuously verbalised. This highlights that no undesired action takes place. Moreover, participants count on previously agreed rules on body parts where haptic probes are to be placed so that explorations do not become too invasive or overly intimate. The result of these sessions lead to a heightened sensitivity towards haptic cues and material affordances. With the soma design approach, the body is inquired throughout the session, as the mechanism to construct meaning but also as the means to evocatively engage with a material or piece of technology. The design space is broadened by body-material insight. It is worth depicting what is meant by the body-material insight. In previous inspiring work developed by soma designers, prototypes like the Breathing Light [Ståhl et al., 2016] demonstrated the ability to switch the users' attention to a body characteristic (breathing) and focus on the experience of inhale/exhale cycles. The 
device counts on a lamp that can dim its light according to a proximity sensor tracking the thorax and the associated breathing cycle. Throughout the design of the interaction artefact - and its usage -, the body is attended and treated with care. That can be accomplished via several techniques or exercises that soma designers elaborate on. With the A-lab, the designing team and users have another route available to get attuned to the body, as it could be the case for the somaesthetic prototypes shown in [Tsaknaki et al., 2019], using it as an extra resource.

\subsection{Affective technology. Interacting with the body}

With the emergence of everyday personal sensing systems, such as those embedded in our permanently reachable phones, smart watches and fitness bracelets, $\mathrm{HCl}$ and ubiquitous computing scholars have highlighted the value of these technologies for innovative research. Technologies that we have seen permeate the everyday space with quantification, exercise tracking, and physical wellbeing, have also -perhaps in line with a more traditional Affective Computing view- made researchers dream of extended healthcare, diagnosis and monitoring applied as well to mental wellbeing. Just to name a few studies, research can be found on the promising field of affective computing technologies for autistic spectrum disorders (see the review [Cabibihan et al., 2016]), on the move towards mobile-based psychotherapy platforms and tracking routines for the youth [Matthews and Doherty, 2011] or the implementation of affective computing techniques in the design of mobile health tools for bipolar patients [Abdullah et al., 2016, Matthews et al., 2015]. As exemplified by Bardram and Matic [Bardram and Matic, 2020], mental health research is catching up. In recent years, research on mobile and wearable technologies that track behavioural, psychological, and contextual signals has gained momentum in the field, albeit not without pending design challenges [Sas et al., 2020]. Pursuing a research path toward ubiquitous technologies deployed in mental wellbeing domains may help to bring to the surface such aspects as personalisation, perhaps achieving forms of rapport or engagement not seen in traditional healthcare. The promise of affective computing, i.e. computing that relates to, arises from, or deliberately influences emotions [Picard, 1997], is vast. In our view, we argue that just as self-awareness plays a major role in the motivation of change in rehabilitation therapy, e.g. in cardiac rehabilitation [Maitland and 
Chalmers, 2010], psychotherapy could benefit from self-monitoring technologies. Awareness, for instance, may contribute both to (re)assessment and behavioural change that are solid grounds of cognitive behavioural psychotherapy. Addressing how technologies expose body characteristics and support (body and affect) self-reflection lies within the core of our goals. Thus, when research in affective technology design is presented, we significantly place importance on emotional awareness. Emotion plays an integral role in design work, and design researchers are not exempt from its ups and downs either [Balaam et al., 2019, Sas and Zhang, 2010a, Sas and Zhang, 2010b]. As affective computing reaches maturity, other ways of approaching affect keep the pace. Links between emotion and physical activity exist, for instance, via dance, exercise, movement, or paying attention to our body senses while immersed in nature. Engaging with the body might therefore be a fruitful path to explore when designing technologies and interactions with a self-reflection scope. There is room for an affective computing that does not look at the body as "an instrument or object for the mind, passively receiving sign and signals, but not actively being part of producing them" - as phrased by Höök when referring to dominant paradigms in commercial sports applications [Höök, 2012]. In an effort to attend to emotions, rather than primarily recognising them, researchers investigating what is known as the affect through interaction prioritise making emotion available for reflection. In such line of thought, seeking emotion aside from context would not make sense. In this affect through interaction exercise, the role that emotion has had for a long time in artistic and design endeavours is acknowledged. This is exemplified by the analysis of Boehner et al. [Boehner et al., 2007], later picked up by Howell et al. [Howell et al., 2018a] to defy the role of personal sensing in the affect through interaction view, in particular the role of biosensing. That is, by no means, to say that the push that personal sensing has witnessed under the advent of affective computing should be diminished. Rather, the dialogue with artificial intelligence research and attention to more cognitivist oriented outcomes can strengthen the affect interaction paradigm. When designing technology-mediated experiences, we view the affect as a sociocultural, embodied, and interpretative construct. 


\subsubsection{When design redefines technology}

Recent examples of Human-Computer Interaction research demonstrate how technologies that so far were relegated to engineering domains, are making their way to design contexts where they are harnessed, twisted, put to the test, respectfully stripping away their meaning to yield new uses. The transformation that design exploration brings to digital materials, for instance Bluetooth communication [Solsona Belenguer et al., 2012, Sundström et al., 2011], paves the way to new explorations on sensing platforms and biodata. These works highlight the relevance of taking an interaction research approach: "it is not enough to experience digital materials at any given moment to grasp their properties and design potentials; instead such dynamic qualities only reveal themselves when put to use. More often than not they have to be assembled as part of running systems for properties to take on form and substance, and especially so for materials as immaterial as radio." The seminal work of Howell et al., capturing the reality of the ubiquitous performance-oriented sensing trend permeating work, home and leisure, points to alternative tangible representations of biodata [Howell et al., 2018a]. Biodata graphs are able to become physical. Biosensing garments present paradigms where biodata is not disassociated from emotional experience, sensing mechanism or display. These are entangled phenomena. Howell builds such case around electrophysiology, where researchers deal with the redefinition of electrodermal activity. Interaction designers, through artefacts, open the design space to gain access to uses that would otherwise be out of sight. Electrodermal activity representations are taken to the extent of exposing properties, creating appealing visuals, or playing with nonhabitual dynamics that do not necessarily require screens. Data is no longer something to check upon, in a disembodied manner, but something that one carries, wears, or enhances in order to better understand the self, engage with, gain knowledge or even give rise to sharing instances. In the work of M. Umair and colleagues [Umair et al., 2018, Umair et al., 2019b], the concept of arousal, also under the electrodermal activity sensing paradigm away from conventional screens, is addressed from the lens of visuals and haptics. In his studies, we observe how tensions like privacy, interpretability and ownership emerge vividly to bear the weight of alternative interactions with personal sensing. In an attempt to go further, building on thermochromic-based displays, skin conductance and its connection to affect still pose questions on how 
users can appropriate the interpretation of arousal biodata and their uses. What if users could drive the design of arousal display platforms? Little by little, the different questions lay the groundwork for the ThermoPixels toolkit [Umair et al., 2020].

\subsection{The ThermoPixels: User-engaging affective biodata}

Affective health and wellbeing has recently caught the attention of HumanComputer Interaction research [Sanches et al., 2019b]. Interfaces that combine physiology with feedback try to promote self-reflection in order to help users be more aware of their own emotions, potentially contributing to their wellbeing. With examples that go from heart rate relaxation artworks [Khut, 2016], to electrodermal activity expressive wearables [Howell et al., 2018b, Umair et al., 2019b] or brain activity feedback interfaces [Hao et al., 2014], biosignals have significantly taken over a particular discourse of emotional awareness. Designed by means of different feedback modalities, new affective technology paradigms coincide in trying to leverage engagement and direct participation of the user in interactions aimed at self-reflection. The idea behind it, is that emotional awareness and regulation constitute basic skills for emotional wellbeing. By emotion regulation [Gross, 1998], we refer to the processes that subjects deploy in order to influence which emotions are experienced, when and how - relating to physiology responses, intensity and behaviours. Commercial devices or research prototypes intertwining users with emotion self-reflection are usually provided to users as closed systems, opaque in terms of working mechanisms, leaving no room for customisation or appropriation. In this research, it is argued that building upon end-user development despite the development difficulties, user involvement entails benefits with respect to agency, attachment and transparency in understanding the technology mechanisms. In other words, end-user development is proposed in order to let the user take actions on his or her own behalf, exposing that the behaviour of the technology is in part fruit of the design decisions taken. At the same time, participating directly in the creation of artefacts or representations exposing inner qualities (in this case, arousal or EDA levels) must enable a closer appreciation for the technology and its uses, with a rapport building on feelings of ownership or authorship that consumer goods do not face. Inspired by the work presented in [Howell et al., 
2018b] and [Umair et al., 2019b], facilitating the reliable measurement of arousal via galvanic skin responses that leads to emotion regulation uses, the question on whether users are able to personalise arousal representations is posed. Can users add their perspective and customise an arousal representation that speaks to them amidst these technology-driven interactions? Beyond the work that $\mathrm{HCl}$ has inspired on combining smart materials and alternative biofeedback modalities which often get tangible or more intimately connected to the user, novel design opportunities for affective technology design are found. In order to find out how the users deal with arousal biodata, in particular EDA, and create representations a toolkit allowing to do so is sought. This is how the concept of the ThermoPixels kit is born. The research described here is a result of a personal collaboration uniting University of Lancaster and PLUX/UJI for a specific $\mathrm{HCl}$ project. The ThermoPixels kit, and the idea of running workshops to assess how users would interact with smart materials to address arousal representations (and arousal itself) were both conceptualised by Lancaster researchers M. Umair and C. Sas. Having collaborated in other occasions thanks to the AffecTech [AffecTech, 2017] partnership, the possibility to actively participate in organising the workshops and analysing the outcomes generated by participants was very promising for this thesis. As it will be seen, this project highlights a different way to bring the user into the centre of the technology interaction. Intersecting emotional awareness as no other study developed within this thesis, the ThermoPixels (based on EDA but not unpacking it explicitly) underscores how different users make interaction (biosensing-based) devices their own, not only addressing arousal concepts on the fly but also questioning their possible day to day uses. Those who build the resulting arousal representations feel connected to them because they own the communication codes and anticipate what to do with them. Appropriation, in a way, should be seen beyond a mere IKEA (self-made) effect [Norton et al., 2012], i.e. the attachment that is created when effort is put in a given product that needs to be finalised. ThermoPixels had an impact in the personal research line that followed, trying to open up the possibilities with respect to biosignals and what they add (not so much concerned final concepts or prototypes for emotional awareness). In this thesis, it contributed to move closer to the study of interaction that uses the perspective of the researchers themselves (see section 4.5, 4.6 and 4.8). With a focus that is put on interesting visuals brought by thermochromic smart materials - i.e. materials that change colour when subject to temperature 
changes - , ThermoPixels unites physical and digital materials that provide visual qualities enabling the creation of alternative arousal representations. Importantly enough, finding out how the toolkit's material affordances are explored and used to create personalised arousal representation regardless of the users' technical skills needs to be investigated. Moreover, not only the wide range of visual concepts or prototypes that emerge from the ThermoPixels needs to be addressed, but also the contexts, hypothesised use case scenarios and their emotional connections, of much relevance to affective technology design.

\subsubsection{Background: Affect, material approach and users' input}

Arousal has been consistently present in discussions surrounding studies of affective computing. Since the early days of the circumplex model of affect [Russell, 1980], affect can be characterised by two dimensions: valence, or the degree at which one rates an experience positively or negatively, and arousal, i.e. the intensity of the emotional experience, ranging from low, neutral to high (see, for instance a simplified version, fig. 4.1). The 2D representation is a tool that has gained prominence in psychology and affect research through the years.

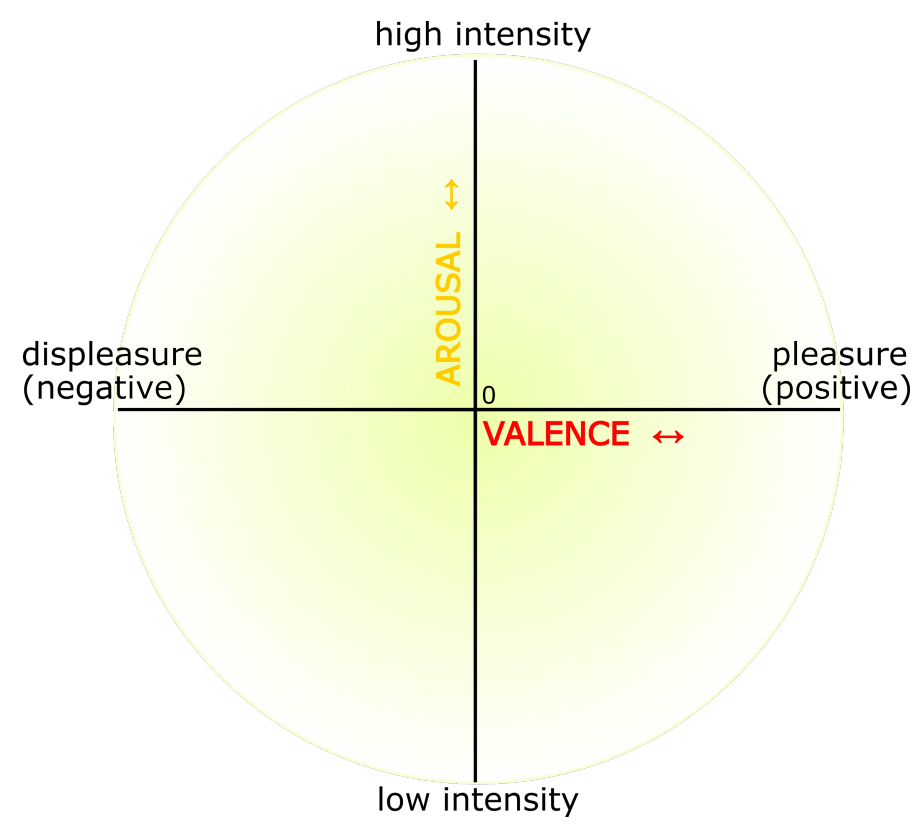

Figure 4.1: 2D representation of arousal-valence affect, where affect words can be placed attending to their intensity and pleasure 
Strictly speaking, Russell and his research on mapping affect concepts in a 2-dimensional circular space referred to pleasure-displeasure as the horizontal dimension and arousal-sleep as the vertical dimension. The representation would be used to judge how well specific concepts such as excitement, depression, distress or contentment would be placed within this framework, along with other words like happy, astonished, angry, sad, bored or serene among others, always attending to their valence-arousal. There is interaction design research in the intersection of wearables, biofeedback and emotion regulation investigating affective interfaces to support emotional wellbeing. A notable example is found in [Miri et al., 2018a, Miri et al., 2018b], where the concept of emotion regulation is addressed via personalised technology, paving the way for the creation of interactive connections - tangibly, with haptics - with the user's body in a way that regulation strategies or interventions are deployed anywhere. In general, the goals of these interaction design studies usually encompass the facilitation of emotional self-awareness and regulation. Emotion regulation technology does not necessarily stay in the awareness scenario only, but should motivate empowering reflection and change, if needed. Other works on tangible platforms, leverage haptics and evocativeness of touch to support clinical mental health interventions [Vaucelle et al., 2009]. MoodWings [MacLean et al., 2013], for instance, targets stress interventions with the use of a wearable butterfly that makes the users reflect on their current stress states. Early works such as Affective Diary [Lindström et al., 2006], explored the combination of sensing and emotional memories in a broader sense that spanned over time, allowing its users to actively own the narrative surrounding experienced emotions and mixing body inputs with personal accounts to reflect and re-assess past recorded experiences. This fertile space, though, gives rise to projects where the combination of emotionally relevant biosignals (such as EDA) with event logging spurs stress event classification and recognition [Ayzenberg et al., 2012] where the active self-reflection role of the user unfolds in a later stage. The work of Affective Health [Sanches et al., 2019a], with a different focus, highlights the interest in long-term data trends, the relevance of open-ended designs where various use cases emerge and exemplifies different uses of colours and shapes to represent arousal. Representations are not necessarily free from ambiguity, which in fact is seen as an affordance of the technology that eventually yields interpretations. These observations, much more in line with the research of M. Umair's material arousal interface research [Umair et al., 2018, Umair et al., 
2019b], allow addressing questions on the users' personal take. This work is notable for building upon smart materials' qualities that provide alternatives to experiment the data. Wiberg calls for a material exploration that works back and forth with the materials in order to understand their properties. This allows one to put materials together in new ways. In particular, "attention to details through a process which switches back and forth between details and wholeness" is sought [Wiberg, 2013], i.e. linking aesthetics to the material details and meaning to the whole artefacts. However, Wiberg draws upon an artefact-oriented perspective [Jung and Stolterman, 2012], therefore assessing what the physical technology brings and not necessarily bound to user-centred efforts that focus on the user's needs. The focus on materiality should not be underestimated, as progress in $\mathrm{HCl}$ research has benefited from it uncovering the experiential and aesthetic qualities of the relationship between user and technology. Although only partially achieved with regard to thermal effects and visual triggers, ThermoPixels tries to build on the envisioned programming of smart materials [Vallgårda et al., 2017], i.e. being able to program changes in the material properties that must unfold during the interaction with a piece of technology. The work of Giaccardi and Karana [Giaccardi and Karana, 2015], enriched the debate addressing how materials (or their affordances) shape interactions, influencing what users do and how users do it. The ThermoPixels, created for EDA arousal representation, aims to address the affordances of the chosen smart materials and let the users unpack them while prototyping with the toolkit.

\subsubsection{A toolkit to design with}

Design toolkits (prototyping kits) containing electronics have emerged in recent years within $\mathrm{HCl}$ research, offering new avenues for tech development and echoing the rise of the do it yourself (DIY) culture [Kuznetsov and Paulos, 2010, Mellis, 2014]. Toolkits usually rely on instructions that guide users in the assembly processes. Some examples of (electronic) DIY kits are found in the context of assistive technologies [Moraiti et al., 2015, Hurst and Tobias, 2011], for instance addressing how this paradigm empowers users with the means to create technologies better adapted for their own uses, at a low cost and potentially increasing acceptance. Other uses involve e.g. end-user programming kits that present different data modalities (light-based and physical-based: visual, vibrational, rotational) aimed 
at environmental data engagements [Houben et al., 2016], and domestic energy consumption tracking [Sas and Neustaedter, 2017]. Targeting the benefits of handcrafting enjoyment and enhanced learning present in the DIY practices, low-cost, thermochromic, smart materials are put into a design DIY toolkit. With the aim of exploring the creation of alternative physiological arousal representations, the novel combination of making and affective technology is studied.

For the sake of understanding better what the DIY designing experience entailed it is worth describing some of the components we included in the kit. Thermochromic materials, carrying notions of range, inertia, duration and responsiveness [Umair et al., 2019b], are the main components of the ThermoPixels toolkit. The material choice consists of black liquid-crystal sheets exhibiting colourful patterns when heated, color-changing thermochromic inks and revealing sheets turning translucid when warmed up (a.k.a. rub-and-reveal). Sheets are bendable, cuttable and provide a sticky side to facilitate manipulation. Inks are provided together with application syringes. The temperature range of operation conditioned the material choice (ranges between $25^{\circ} \mathrm{C}$ to $40^{\circ} \mathrm{C}$ ), in order to obtain relevant responses without compromising safety. Heating materials using electric current to deliver temperature to the color-changing components are also part of the toolkit. Nichrome wires, heating pads, thermoelectric coolers (Peltier modules) and conductive fabric are used. Besides, the Arduino-compatible Grove GSR sensor [Grove - Seeed Studio, 2014] for electrodermal activity tracking is the pillar to capture the arousal changes. The sensor, tracking changes in skin conductance due to the sweating that the autonomic nervous system drive in the presence of affect stimuli, is used alongside an Arduino board and basic electronic connectors (cables, alligator clips, etc.). The electronic system is enclosed in a plastic box and ready to be connected to the prototyped displays. Other materials accompanying the kit components are mainly for isolation (plastic sheets, cardboard, etc.), sketching and crafting facilitation purposes (colour markers and sheets to create the main visuals, scissors, cutter, glue, Velcro, adhesive tapes, etc.). A relevant part of the design leverages colour contrasts, the interplay between opacity and transparency, and the layered structure that the heating and visual components will impose. Each of the heating elements presents different affordances, i.e. weight, rigidity, current-temperature dependence, temperature dissipation properties, etc. In order to not overwhelm participants with choices, it was decided that every participant would exclusively work with a single (randomly-assigned) heating element without 
having a choice. At the same time, evenly distributing the heating elements would ensure that no bias with regard to heating choice would arise, e.g. ending up with too many fabric-based outcome prototypes due to the resemblance of its characteristics with those of wearables.

Once the kit was set, we set out to run the experimental phase of the study. In order to study the usage of the ThermoPixels toolkit, hybrid crafting workshops of 3 h duration were organised by our team. Every workshop hosted 2 participants who worked independently. 20 participants (average age 27; 9 women), compensated by a $£ 30$ worth voucher on online purchase credits and recruited through a university campus, ended up being part of the study cohort, yielding to 20 different outcome artefacts ( 5 prototypes per heating element). A researcher is available all the time in order to provide technical support if needed, i.e. ensuring electrical terminals are safely connected and operating power supply. At a workshop, a participant follows 3 stages to embark on the design of her/his own arousal representation:

1. Being introduced to arousal and the DIY toolkit. Sketches and low-fi prototype concept.

2. Crafting. Design work and prototyping. From drawings of arousal representation to prototyping thermochromic visuals.

3. Testing the prototypes. Reaction to stimuli and arousal triggering.

Emphasis is put at introducing the concept of arousal as emotion intensity. After sketching several representations of emotions at different levels of intensity, participants are asked to think of a unique image that conveys the increase of arousal (not necessarily bound to a discrete emotion) and imagine how it could be translated to a low-fi prototype with the given materials. 

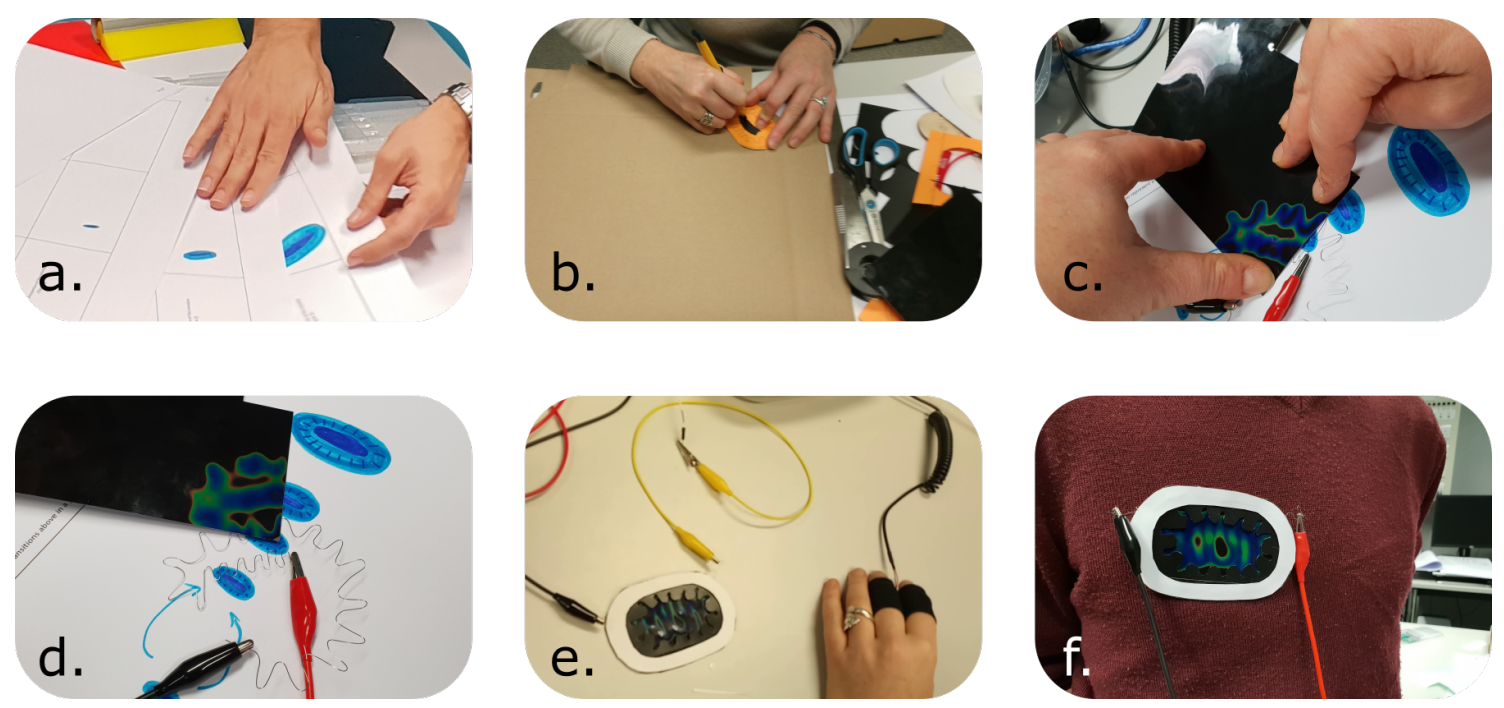

Figure 4.2: Crafting arousal representation prototypes with ThermoPixels. Badge: (a) sketching, (b) layers, (c) thermal/touch exploration of liquid crystal layer, (d) connecting the nichrome wire, (e) arousal EDA sensing test, (f) wearable badge

The sketching stage is of particular relevance, as participants start to inadvertently experiment with the connections they establish between different emotions and the various colours and shapes, to end up transitioning to the concept of intensity per se that will be translated into a prototype that needs to convey arousal change. This activity potentially saves trial and error time and orients the toolkit's material exploration that follows. The prototyping stage takes inspiration from previous research on the layered structure of crafted thermochromic-based platforms [Umair et al., 2018, Wang et al., 2017]. Participants create the objects that will hold their arousal representation and host the heating mechanism activating the visual effects (see examples in fig. 4.2 and 4.3, where e.g. the nichrome wire is used in combination with liquid crystal and revealing sheets to create, respectively, a badge and a desk air purifier/paperweight). The activity involves substantial trial and error for both implementing the sketched concept or pattern and experimenting with the material affordances of the different components. Finally, the activity concludes with the opportunity to test the mechanism and visuals created in the form of an arousal prototype by means of a set of IAPS [Bradley and Lang, 2017] arousal-triggering pictures shown on a laptop screen. 

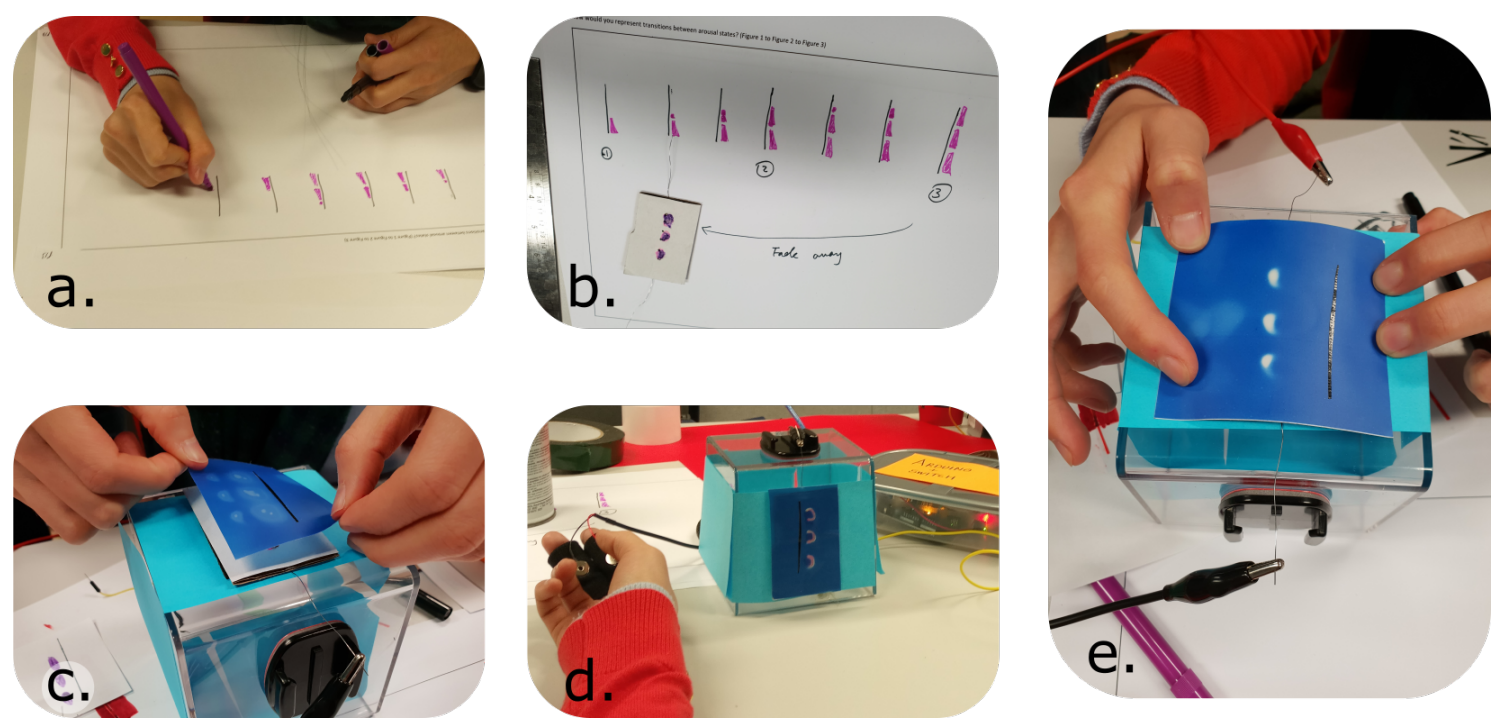

Figure 4.3: Crafting arousal representation prototypes with ThermoPixels. Flowers: (a) sketching, (b) arousal transitions, (c) layer structure/revealing sheets, (d) arousal EDA sensing test, (e) nichrome wire heating

\subsubsection{Prototypes. Affective technology design lessons}

A key factor of the ThermoPixels is that the users who created prototypes with it explored the materials from an embodied perspective, spontaneously. The thermochromic materials prompted touching, breathing on, squeezing, folding and stretching the components. This exploration informs the participant about the material responsiveness, colour aliveness and time dynamics. Although not directly addressed, these body-led affordances could reinforce the attachment to the created representations emerging already from the DIY craft journey. Thermochromics posit themselves as relevant candidates to drive further affective research. The experience of participants, addressed via the created artefacts and a qualitative interview, indicate that complex affective technologies as the ones deployed in ThermoPixels achieve a certain level of accessibility regardless of participants' background or field of studies. The toolkit significantly enables to input user creativity into otherwise distant technologies. The outcome prototypes are vast, exploiting concepts such as a projector, a mobile phone case, a ring, a pair of sneakers, an air purifier, an alarm-like clock, a dynamic badge, a wristband, or a lamp, among others. Notably, wearables constitute a relevant amount of the outcomes, but circumscribing affective technologies (or interfaces for arousal 
reflection) to wearables would be an error. Fortunately, the ThermoPixels was not addressed in such a manner. The internet of things ecosystems that are found in today's societies allow the emergence of other paradigms, as it could be the case with the smart agent present in the ThermoPixels' outcomes, the air purifier, the projector or the clock. All of them present interesting features like portability, or are subject to attributes like smart, connected or responsive technology. As a learnt lesson, finding the media that best speaks to the user should not prevent these fruitful explorations that place affect reflection concepts beyond wearables seen in otherwise meritable research [Umair et al., 2018, Howell et al., 2018b, Devendorf et al., 2016]. ThermoPixels' prototypes, due to the way they are built, open the way for inputting creative personal expression, something that contrasts with other DIY studies. The chosen smart materials are a vehicle to open-ended exploration via hybrid crafting [Golsteijn et al., 2013] - combining the physical and the digital-, as opposed to assembling instructions. The body, with not much attention paid as it is often the case in interaction design, is not directly addressed from the toolkit design. However, the use of the body emerges vividly in the crafting practice, revealing how participants leverage their bodies as actuating tools triggering temperature effects. This adds to the aforementioned embodied or haptic take on material exploration. In doing so, the users establish connections with the materials that go beyond colour and shape choices, potentially replayed when the arousal visuals are triggered in future use case scenarios. This aspect should reinforce the idea that the role of the body needs a place in affective design work, with so much potential insight to be studied, i.e. with biofeedback and emotion mappings to be explored in whatever novel affective interaction is created. Moreover, workshop participants navigate repeatedly from body-as-input (through the sensor, through the triggering actions to explore the materials) to body-as-output (via effects experienced on artefacts attached to the body). Affective technology design should consider the benefits of addressing this body-material interplay before any development is deployed at a large scale. Creating space for open critique touches, at the very least, foundations on the ethics of technologies that touch upon users' emotion. This body role, we argue, could be paired with research studies like [Lazar et al., 2018], where Lazar et al. suggest that there is room to explore materials as language potentially overcoming expression difficulties.

Looking at the results of conducting the ThermoPixels workshops, an aspect 
regarding the use of the prototypes is worth noting. Participants either conceptualise the prototypes as artefacts for emotion expression or technology for emotion regulation. Both uses are crucial for the effective functioning of everyday life and emotional wellbeing. Emotion regulation is continuously at play, for everyone. Its importance can be illustrated with examples. The accounts presented in the context of project WEHAB [Miri et al., 2018a], for instance, speak about the impact that emotion regulation has for emotionally charged occupations such as flight attendants, police officers, customer facing services, military personnel, and emergency response personnel. For them, it is very important to detect when negative emotions rise and select a coping strategy accordingly. The example text goes on to hypothesise how technology could privately cue users with appropriate emotion regulation strategies, warn about overreacting instances or motivate changes of coping strategies. The chosen occupations make it easier to empathise with the effects that emotion regulation may have when dealing with customers, suspects, opponents or patients under stressful situations. However, emotion regulation affects everyone. ThermoPixels outcomes, notably, allow users to hypothesise with emotion expression and regulation mechanisms, two aspects that are not necessarily addressed together in affective technology research. In terms of arousal depiction, despite the limited number of prototypes, outcomes show an important variety of choices of colour, softness and size of geometrical shapes, but also going beyond and moving from $2 \mathrm{D}$ to rich $3 \mathrm{D}$ forms. Moreover, this study could motivate further research on the associations that emerge between emotion intensity depictions and colour, roundness or shape choices, feeding into previous research reflections, e.g. [Khut, 2016]. Beyond the effect of the affective technology assembled, there is potential to use the toolkit as a psychoeducation tool. Not only the toolkit attunes the subjects encountering these sensors and effects for the first time but can also help affective technology designers being introduced to novel platforms or materials of interest for their practice. Participants note that the use of the ThermoPixels sensitise them to the challenges and skills of emotion regulation, setting the ground a mindful consideration of emotional needs and use scenarios discussion. Through the use of the different materials, although not infallibly, participants better approached the concept of arousal as emotion intensity, opposed to negative-positive valence and not necessarily coupled with discrete emotions. Material limitations and the frustration when desired visual effects were not achieved dominated the experience. The challenges posed by an 
EDA sensor measuring galvanic skin responses were foregrounded too.

\subsubsection{Limitations}

The ThermoPixels toolkit, at a small scale, has worked to unveil design possibilities, body-material affordances, and opportunities for psychoeducation and affect-tech design support. However, conclusions are drawn from a very small population, with mostly higher studies backgrounds. The design insights obtained surpass those of previous developments and pave the way for future research. Furthermore, no long-term use has been addressed. In a particular single case, doubts remained on the working of the sensor even after prototype testing, confusing temperature events as sensor triggers instead of skin conductance fluctuations. The processing of the data, mainly based on an average threshold calibrated during the prototype trials, still has room for exploration in terms of event recognition, trigger times and recovery dynamics. Moreover, with regard to toolkit contents, while the ThermoPixels kit is clearly delimited by an EDA sensor, the thermochromic materials and the heating elements, the supporting materials open the door for an ever-growing list of insulation components and crafting support tools that render difficult the analysis of the hybrid crafting toolkit as a standalone set without the aid of any researcher. All in all, this study paves the way for future research in affective technology design, either deploying DIY kits, leveraging the user's customisation, addressing psychoeducation goals or taking the chance to focus on the body as a vehicle for experience and meaning-making from the early stages of the design.

\subsection{Designing from a first-person perspective}

\subsubsection{Embracing discomfort}

Research such as [Sanches et al., 2019b], has called attention to the rise of affective technology development within $\mathrm{HCl}$. Although the field has experienced great advances in the recent years, unclear or nonexistent information on the ethical foundations behind affective developments and applications pose serious concerns - on current research falling short, difficulties for designers to properly attune to vulnerable users, and broader risks if technology approaches 
technology-driven businesses without solid evidence-based grounds. First of all, the nonexistence of an ethical framing or disclaimer in a given affective health study within $\mathrm{HCl}$ shows a certain distance with respect to the users. When considering cases in which targeted users present vulnerable conditions - such as those belonging to a user base with a given affective disorder diagnosis -, an ethical framing of the designer should be of utmost importance. Such vulnerabilities could easily touch on aspects like exposure, failure to provide support or data privacy. Some would argue that non-maleficence - the goal of committing to harm-free studies - is a basic, shared value within $\mathrm{HCl}$ research, inherent to the discipline, the challenges posed by the study of tracking technologies and the ubiquitous data ecosystems present nowadays set the bar significantly higher. Under these terms, non-maleficence may translate into assessing data privacy provisions, into a guarantee that users are accompanied by professional or medical resources throughout the study and a need to understand what a regular use of technology feedbacks and reminders of a given condition implies. Non-maleficence may, therefore, not be sufficient, having to rely on other ethical principles such as beneficence or fairness. Accordingly, a designer must go through an ethically sensitive evaluation during the process of creating an artefact or interaction. Moreover, technologies can be appropriated in harmful ways. The weak ethical ground highlighted by the body of literature analysed by Sanches et al. is even aggravated if one considers the commercialisation of mobile applications, a business that could easily frame $\mathrm{HCl}$ research and technology in affective health domain, too often lacking evidence-based foundations, without transparency on methods, therapies and principles followed.

Having had these considerations, we turn to design approaches that may provide the means to engage with the technologies' often overlooked ethics when embarking on such designs, without necessarily engaging vulnerable populations at an early stage. First-person design [Höök et al., 2018, Lucero et al., 2019], offers a relevant alternative to engage directly with the experience of the designed artefacts and interactions in order to learn, first-hand, the impact that the designed experiences have (section 4.2.2). In this section, work developed in the context of the present thesis, discussed in [Umair et al., 2019a], is presented, motivated by a UbiComp design workshop trying to get opportunities from reassessing uncomfortable design [schraefel et al., 2019]. When using first-person perspective, what is sought by us is the effect of leveraging the body role in perceiving the 
experiences, their aesthetics, and not only its use as input where data is to called to reveal body-emotional characteristics but also as a body that is a sentient output receiving the effects programmed in the technology. In particular, the interest in the research presented here lies in the use of wearable physiological sensors capturing electrodermal activity and conveying meanings on arousal. These affecttech sensors are not free from usage challenges. With first-person design, the opportunity to step into the users' shoes offers the possibility to probe the ethical challenges that affective technology and interaction design pose. Moreover, in line with studies that exemplify biodata engagements not necessarily linked to quantification goals [Howell et al., 2016], first-person design offers alternative narratives in a space that too often gets caught in the biosensing pursuit of performance-based self-improvement interactions impacting how we feel, move or behave in order to achieve a (supposedly) healthier life.

Two design experiences with the body are the focus of this study. The idea behind our approach is that in order to more actively engage the body into the design of affective technologies, inspiration can be spurred by borrowing ideas from body-centred practices already informing the design practitioner. This is the case of Contact improvisation dance and Yoga. Can such practices inspire reflection on the lived experiences and inform the design of technology interactions? The engagement with the mentioned activities, takes place in groups, hand in hand with professional conductors of the two body practices:

1. Contact Improvisation: Contact improvisation dance [Smith and Koteen, 2019] is a form of dance that stresses the properties of touch and weight sharing. With the help of a partner, contact touch points are explored to spur movement improvisation that is centred in the present, flowing nonjudgementally. Engaging personally in these exchanges, regardless of level of expertise, age or gender, non-verbal body negotiations are explored. What are one's limitations? What is it possible to do? How far can one push? These are boundaries and intentions that are explored by the mere subtle touch pressure of bare contact -sometimes aided by gaze too. When in front of dance partners, one gets to see the own body as if looking at a mirror. Being in contact reveals sweaty body parts, clumsy and shaky movements. Speed changes, directions and backward or forward advances are communicated through touch. The subtle meanings involved are not easy to convey. An uncomfortable insecurity sets in. 
2. Yoga: Yoga, is an ancient Indian tradition aiming to bring physical awareness to the body. For an interaction designer, yoga offers valuable tools to address proprioception and the felt body. When practised among other researchers, all with different skill levels, we felt that trying to cope with the conductor instructions highlighted the notion of comfort. Our bodies were not capable of comfortably follow the routine. Suddenly, we lose grip of the required nonjudgemental state and fall, sweat or get embarrassed. Discomfort evolved from the exercise frustration to the physical domain with aches and heightened body awareness. Yoga probes our responses, our limits and makes one confront preconceptions we have about our bodies.

\subsubsection{Electrodermal haptics}

As exemplified by the Grove [Grove - Seeed Studio, 2014] electrodermal activity sensor used in the ThermoPixels (see section section 4.4.1) [Umair et al., 2020] via finger electrodes affect is often measured in terms of arousal by using the emotional intensity. Inspired by the material trend in $\mathrm{HCl}$, the use of actuators responding to captured biosignals in everyday settings is of interest. These are technologies that touch the body, sometimes literally attached to it. The use of off-the-shelf components such as little vibration motors allow the implementation of a simple but evocative vibrotactile EDA biofeedback system, using a galvanic skin response sensor. In order to probe the implications of using the system, a simple sensing-actuation prototype is placed on a wrist and addressed for a period of 2 days of use. How do changes in EDA feel in terms of vibration? Is the sensor comfortable? On the first day of use, a few minutes of wearing the finger electrodes are sufficient to reveal certain incommodity. The sensor is tight and needs to be loosened. More difficulties emerge when for instance trying to do everyday activities requiring both hands, as it is the case with typing on a keyboard regardless of the dominant hand. Doing so, triggered the vibration something completely unintended related to erroneous readings. A similar effect was encountered when opening a metal door. As it turns out, different actions lead to relevant skin conductance fluctuations. On the following day, an awkward experience took place during a visit to the supermarket, where people began staring at the low-fi looks of the prototype and questioning what it was for. 


\section{Discomfort as a design element}

Discomfort emerged as an element of the real-life interactions. The uncomfortable becomes an ingredient of our design research. Recurrently, discomfort appears when adopting a first-person perspective. Our lesson for the design of affective technologies is that, if discomfort appears amidst the experience of experienced researchers it is not hard to imagine that end users wearing the technology would struggle with similar issues. Rather than pushing discomfort back, we see it as an opportunity that first-person research puts forth for the researcher to probe preconceptions, programmed effects and reactions that only take place under daily use cases. Its use unpacks ethical dilemmas without putting much at risk. To what extent is someone prepared to face the information that tracking technologies make visible? How do technology users react to feedback that is continuously available, about our internal states? How does this translate to the experience of vulnerable populations, such as those suffering from affective disorders? How would they cope with faulty technology or outputs? These ubiquitous technologies expose us. Parallels can be drawn between these reflections and the ThermoPixels study (section 4.4) or the $\mathrm{HCl}$ designer responsibility referred to in the work of Sanches et al. [Sanches et al., 2019b]. Moreover, a first-person approach allows the designer to use discomfort as a way to accompany the learning process of the user, opening the design possibilities or construct new meanings around affective wearables.

\subsection{Dare to design: Sensory misalignment}

Continuing with the inspiration that first-person design approaches bring to interaction design, a view that motivates experimenting first-hand the technologies and interactions designed, the question of whether novelty can emerge from the intersection with other design stances pushing the boundaries of the body is posed. At the opportunity of conducting a joint exploration with the University of Nottingham's Mixed Reality Lab, skilled in Sensory misalignment, the challenge was accepted without hesitancy. The KTH Interaction design team I was collaborating with, embarked on a novel exploration. This research is the result of an encounter of Soma Design ([Höök, 2018], section 4.2.3) and Sensory misalignment [Marshall et al., 2019]. Sensory misalignment research has risen 
in recent years due to the renewed $\mathrm{HCl}$ interest in $\mathrm{VR}$ platforms. In sensory misalignment, engaging experiences are created by means of the manipulation of perceived information gathered through the senses. This information can relate to the senses of kinaesthetics, i.e. body motion, orientation, equilibrioception (balance), proprioception, audition, temperature, touch (haptics), smell and taste. Sensory alignment should be understood in interactive systems as the consistency of the main sensory input highlighted by an interaction and the rest of sensory inputs gathered during the experience. Its opposition is what receives the name of misalignment. Often, parallels can be drawn between sensory misalignment and the rubber hand illusion [Ehrsson, 2005], an experiment that has motivated research in VR experience creation and neuropsychology studies. In sensory misalignment, the interest of working with senses that are purposely not aligned varies from the obtention of novel, thrilling or different entertainment experiences to the possibility of administering interactions that reorient how actions are conducted by a user (e.g. redirected walking [Razzaque et al., 2001] or Byrne et al.'s AR fighter game leveraging disorientation [Byrne et al., 2018]). The sensory misalignment design team counted on a background assessing digital stimulation for sensory alignment that integrates insight from practices beyond $\mathrm{HCl}$ [Marshall et al., 2019]. Soma design, which thrives with its "disrupting the habitual" attitude see defamiliarisation [Bell et al., 2005] -, could indeed be extended by sensory misalignment expertise. The encounter of the two approaches may help address how and why misalignment experiences are designed.

Especially in the context of VR, sensory misalignment is often seen as a negative property of systems, where links between VR motion sickness and mismatched physical motion are found. However as argued by Marshall and colleagues, in certain circumstances misalignment might serve the creation of novel and positive experiences, getting rid of limitations that hinder the exploration of a wider range of experiences. Two of the misalignment application domains are the application of techniques that bypass technological limitations (as for instance using subtle cues that achieve an experience of a long VR walk when the technology is limited by its use in a small indoor space) and the deployment of misalignment for Vertigo play in entertainment (creating sense conflicts that enhance certain aspects of the virtual game and makes it more thrilling). A workshop (2 days) on sensory misalignment with soma design was prepared. Prior to the design sessions, the topic of balance was chosen in order to provide some context. Balance is a 
multi-sensory experience, involving touch, vision, proprioception and vestibular sense mechanisms [Byrne et al., 2018, Day et al., 2002]. For the participating researchers, emphasis was put in the body affordances that balance evokes through the examples of the VR flying harness and Balance Beam that the hosting institution had set up to explore. The VR flying harness can be seen in fig. 4.4. The workshop counted on other proto-experiences such as Dancing prosthetics or Balance guitars that fall beyond the focus of the research presented here. The soma design team, equipped with body-centred practices (contact improvisation, Feldenkrais, body scans, etc.) and tools to reflect upon the body, prepared activities to address balance aside from technology. An early remark consisted in re-purposing balance as state to be at, i.e. being in balance as opposed to holding balance. This was complemented by the Soma Bits design toolkit, offering evocative shapes and haptic actuation platforms (Arduino-based heat and vibration modalities) that help connect effects to the body [Windlin et al., 2019]. Participants had also access to PLUX's BITalino physiology monitoring platform [BITalino, 2013, da Silva et al., 2014].

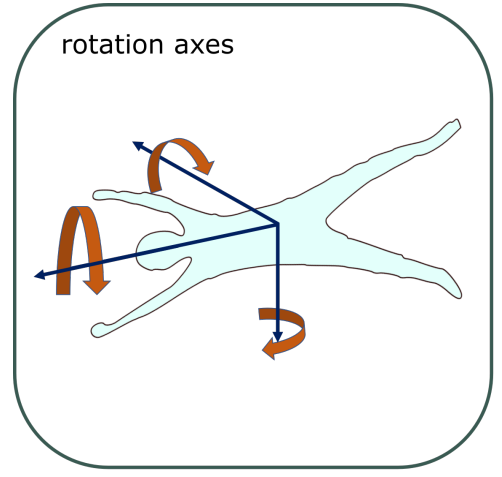

a.

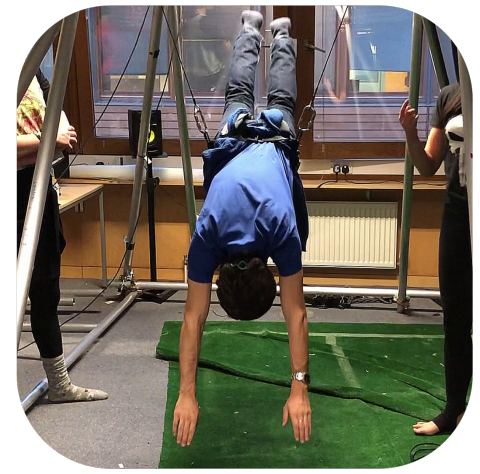

b.

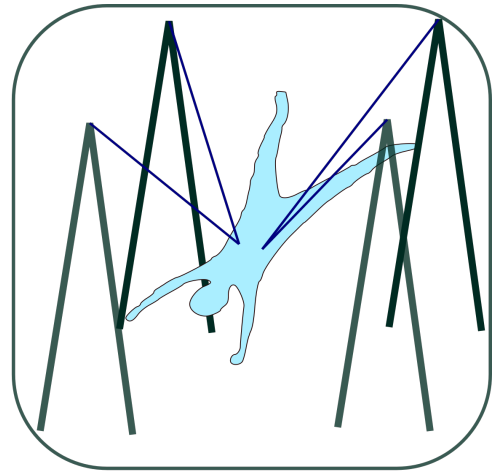

c.

Figure 4.4: VR harness depiction, with (a) rotation axes, (b) real example flying downwards, (c) VR scaffold position

\subsubsection{First-person account insights}

When embarking on this design exploration, one wonders what the complementary design approaches would bring to one another. The free exploration format of the soma design sessions allowed participants to easily go from visual-based disturbances of balance to richer misalignment inputs such as shapes touching the body, with different softness degrees or even offering digital-enabled temperature 
and vibration qualities. Whereas visual based misalignment is present in VR research, used to achieve movement effects in VR entertainment, there was no need to stick to this dominant paradigm. Blindfolding participants to draw attention to the somaesthetics offered by other senses, or addressing misalignment by limiting participants' movement - e.g. on a balance beam or suspending them in a VR harness that changes how weight and movement are perceived - are strategies that, as it turns out, can complement each other. Adding noise and cutting the interaction flow may be seen as a bad idea. However, doing so, forces the participant to reconsider the body action that she or he is carrying out. First-person experiences in a group do not necessarily coincide, as it can be seen in aspects of the interactions approaching discomfort:

- Familiarity and skill: One of the aspects that emerged remarkably when attending to the different first-person accounts derived from the workshop is that skill level plays an important role in how users experience the balance activity. The extent to which someone is acquainted with a practice influences which senses are foregrounded when misalignment sets in. Moreover, different degrees of coping with the misalignment were observed with respect to expertise. Misalignments often had a greater impact on participants who were acquainted with the balance practice at hand, making it more difficult for them - the experts - to beat or overcome successfully.

- Inside / outside: Certain activities, such as placing sensors and actuators on the body of people who is in experiencing the misalignment of their senses in a way, exposing a degree of vulnerability - exposed body boundaries on what one is ready to do, i.e. placing devices on clothes, in close contact to our skin, perhaps within our bodies, while being observed, touched or guided by someone.

A long-time concern of soma and first-person design is how to share experiences if everyone is unique in the way interactions are experienced. Even when engaging deeply with the way our bodies perceive interaction, how can insights be shared? The group design workshop presented here unavoidably confronts the pluralism of experiences. By means of this workshop, the value of the materials used to spur body-centred reflection and account for the other peers was strengthened. And these would be materials that would stick to this thesis' $\mathrm{HCl}$ efforts. Sheets where body sensations are sketched, labelled and reflected upon, group discussion 
sessions, the open-ended design scope and bearable pace that soma design imposes ends up foregrounding the different subjectivities. Bodies are inherently different. Size, weight, skin colour, age, strength and physical capabilities differ. Inevitably, how participants experience emotions, understanding, empathy for the others and meaning making around an interaction, become part of the group discussions.

The somaesthetic and misalignment research used topics to let first-person experiences emerge. And balance proved to be a good nonindulgent topic choice that certainly unveiled differences. Everyone was touched or puzzled by it. Rather than interpreting it as a cause for setting red lines, or contributing to see design collaboration as a harsh effort, the soma exploration of balance encouraged sharing and empathising with the experiences of our peers. Diverging subjectivities should teach us the need to care for every potential user and her/his needs and motivations, somehow resonating with the ethical aspects underscored in section 4.5.1. The elements that supported our misalignment research encounter became tools to pave the way for further interaction design research where the body is purposefully addressed, as it is the case with alternative affective technology designs this thesis advocate.

\section{Balancing on a beam}

The virtual reality balance beam proto-experience, consists in a challenge to cross a balancing beam (or pole) while wearing a VR headset that presents a virtual scenario. The VR adds orientation misalignment possibilities to probe how the concept of balance is disturbed. Different material balance beams enable the exploration of different levels of difficulty (softer, lighter, more slippery, more rounded) for the experience. Moreover, extra tools such as the aforementioned biosensing platforms (providing e.g. EMG - muscle electromyography tracking) and actuation technology let designers enrich the experience and explore different sources of misalignment beyond visuals (vibration, temperature). Balancing expertise emerges as a crucial aspect of the interaction, impacting how participants react to the different misalignments, usually having a milder effect in those less acquainted with the balance practice. On the contrary, VR visuals that hide the feet provide a stronger effect in the case of the novice user. As put by a skilled participant: "l've been able to walk tightropes and balance beams for years, but 
when someone put the vibration on my foot, suddenly I was like a beginner again. It made me realise quite how important foot feel is to my balancing, in the same way hand feel is vital for hand-balancing."

Furthermore, the design exploration, always counting with this drive for the nonhabitual, led on occasions to remarkable interaction additions: "While I balanced on the tightrope with the EMG sensor attached to my calf, the rest of the team watched the screen showing the realtime plot of the EMG signal. We cycled through different calf muscles until we found the one most distinct. [...] In a second session, we redirected the sensory data to music software that mapped the intensity level of the EMG onto the pitch of a sine wave. While balancing on the beam, everybody could hear the changes of my contracting calf muscle. This changed the perception of my balancing act."

This shows ways to enrich somatic engagements with data, or even open perspectives to make biosignals more available or linked to the body, a topic that is later addressed in depth (see chapter 4).

\section{Suspended on a VR harness}

The flying harness proto-experience consists in a flying harness that holds the weight of the researcher, suspended by the hips, so that VR superhero-like flying experiences can be prototyped. Wearing the harness and a VR headset transports the user into a city scenario where two modalities of flight are simulated: one based on hand-propulsion (hands pointing down) and one based on upwardfist gestures pointing at the direction aimed. The use of the harness conveyed the qualities of being suspended, allowing to address the somaesthetics of the different movement framework. At some point, VR was deemed unnecessary to drive a design exploration, given that simple blindfolding paired with the harness offered countless opportunities to leverage body self-awareness. As in the case of the balance beam, there was the chance to use biosensing platforms and components from the Soma Bits toolkit (shapes and actuators). In the harness, however, no narrative was ever used. Hence the VR harness might even exemplify more vividly the usefulness of open-ended group explorations that try to leverage our somaesthetical appreciation. The exploration of suspension together with interesting haptics provided relevant design insight. As it can be seen in personal accounts of the flying harness experience, while everyone found mechanisms 
to connect to augmented body suspension interactions, the design exploration unveils thought-provoking effects but subjective differences too. These differences were sometimes relevant for participants to connect together, to empathise. An example is found when applying vibration while being suspended. Oftentimes, discussion emerged on how the different subjective sensations need to be taken into account. As put personally: "[...] when my colleagues applied vibration on my neck, I lost a hold on where I was. When approaching my back with a soft firm surface, unexisting arms seem to grab me softly." Another participant is quoted by saying: "The most interesting, and at the same time confusing experience, was the illusion that the vibration applied on my back created. I felt spinning around myself and slightly dizzy. My sense of balance was totally distorted and confused and I tried to stay completely still to limit this sensation. For a while I did not know whether I was actually moving slightly back and forth, trying to divert my attention from feeling like I was spinning; or whether I was standing completely still." These accounts underline the potential of somatosensory exploration in groups, and how experiences like these enrich the design of interactions, making designer/users connect bodily with technology-driven effects and challenge emotional, physical and social boundaries.

Regardless of the preparations prior to the workshop (with the preparation of dedicated body-centred practices addressing balance), it is not until one experiences sensory misalignment that the somaesthetics of the experience let one understand what works and what does not in order to create the opportunity to evocatively disrupt the habitual experience and re-engage with a balancing action. Sensory misalignment, resonating with the idea of estrangement, does indeed contribute to soma design methods, encouraging designers to go beyond prevalent soft, comforting, and soothing effects to embrace discomfort, disturbance and confusion that yield a wide spectrum of interaction possibilities. Despite the agreeable pace at which soma design usually unfolds - "we design slowly", as put in the Soma Design Manifesto - and the fact that soma design enables, notably, body and somaesthetic self-reflection, nothing prevents a somaesthetic exploration from engaging with other disruptive qualities. Sensory misalignment teaches so. Staying with the design topic and proto-practices for the 2 workshop days and the subsequent analysis, enabled a deeper reflection on the overall aesthetics of the design experiences. The narrative around the EMG experience, for instance, helped hold the pieces together, i.e. the interest in pushing the balancer off the 
comfort zone, the use of haptic components making participants focus on their bodies while misleading the senses of the expert balancer and the use of a sensor unpacking an internal body insight shareable with the design team. How does the interaction travel back and forth from aligned dynamics to sensory misalignments? The research presented here unveils the need to orchestrate interaction trajectories within levels of familiarity, comfort/discomfort, or even exposure, using technology crossing the boundaries of what is inside or outside the body. Interaction trajectories need to be orchestrated not only through narrative, but via digital effects, emphasis and emotionally charged passages engaging the users' attention by different mechanisms such as fear, reward, excitement, disorientation and pleasure.

\subsubsection{Limitations}

As it turns out, interests of the two design labs involved in this study counted on several intersections. In the end, soma design has enriched sensory misalignment and sensory misalignment has enriched soma design methods. Remarkably enough, spending time with other cutting-edge interaction designers lets one understand their motivations far better than through the reading of published works only. While this research example, built upon the similar design groups' interests on body design and engaging with the non-habitual, is a call for thoughtful explorations encompassing different design teams, what has worked for us under this format does not guarantee success for other groups. The coexistence of different design approaches is rich, but leads circumstantially to design tensions that are not necessarily easy to foresee or deal with. Coming from a soma design stance, where I often embrace provocation as a tool to either rework on the grounding of my design approach or elucidate ways to progress, is helpful. However, designing with the body is faced with culture, physical, and mental boundaries that everyone carries. If interested in affective technology design, the examples found in these somaesthetic encounters should hint that the benefits of addressing these kinds of subjective appreciations are relevant enough to consider first-person explorations before any technology is deployed for end user manipulation. 


\subsection{First-person Physiology: The Sensing Self}

In recent years, physiological monitoring research has been gaining prominence. Drawing upon the do it yourself DIY culture inspired by successful platforms such as the Arduino and the RaspberryPi, specific low-cost platforms that foster an ever-growing ecosystem of multiple devices, multiple programming interfaces and the paradigm of ubiquitous technologies have emerged in the domain of personal biosensing. At the same time, interaction design has witnessed the emergence of first-person perspectives leveraging the designer's experience. In order to facilitate a first-person exploration of biosensing, a set of recommendations is laid out before design sessions. Used as tutorial materials or, rather, suggesting prompts, the group experience guidelines and visual representations presented are aimed at designers, researchers and students wanting to get attuned -with their own physiology- to an otherwise disembodied material. Below, EMG, ECG and EDA examples are shown.

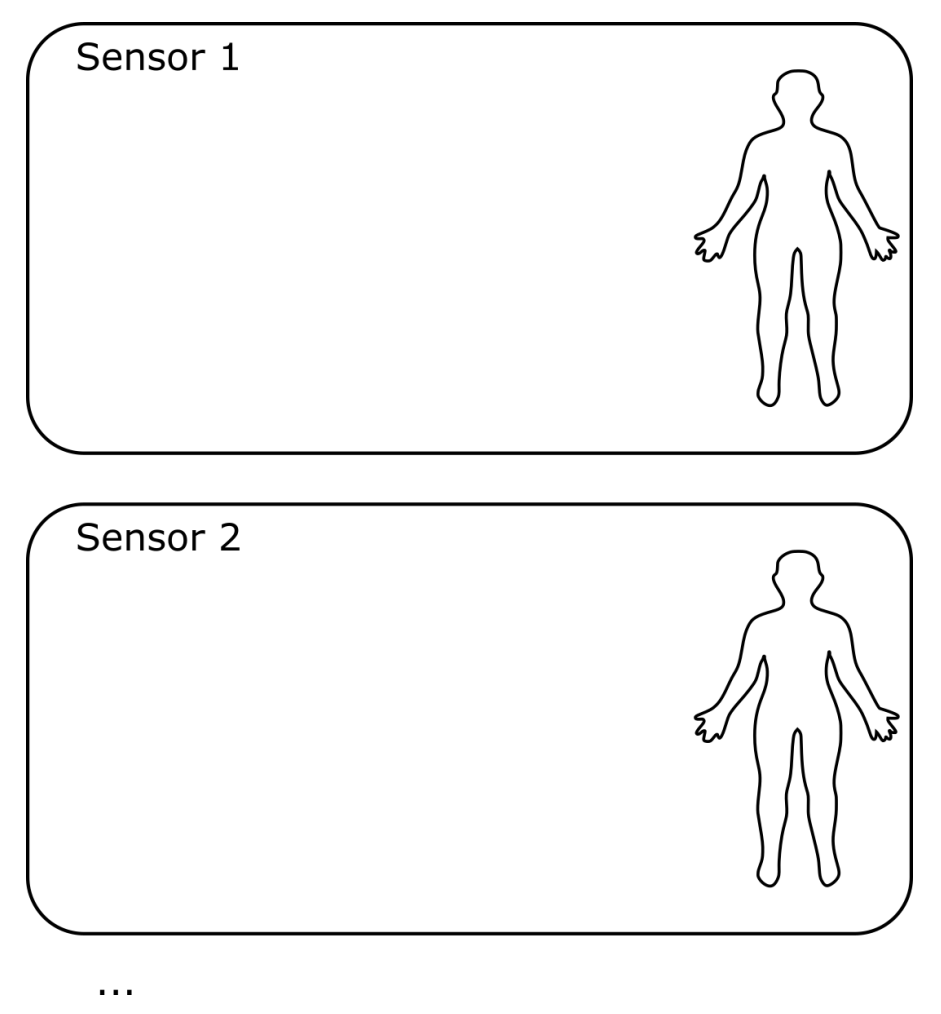

Figure 4.5: Body shapes to reflect on our own limitations, sketch sensor placements and decide involved body parts 
The idea is that the biomedical engineer or the interaction designer can build on such examples, appropriate them, and adapt the biosensing tips to forms that better suit their design frameworks or goals. Along the lines of research presenting first-person exploration experiences touching the body, roles and agreements can be arranged prior to the exploration session so that trust is built between e.g. session conductor and (sentient) participant. The exploration has to spur novel ideas, use cases, sensor placements and non-habitual ways to relate to sensing. However, those involved in the session must ensure that participants have the means to stop if the experience gets too invasive or uncomfortable. For that purpose, a conversation is held before the session to respect the decisions on either what sensor is to be explored, what body parts will be used, or what actions can or cannot be asked to be performed. Bodies are different, and users hold different views on what is considered emotions, behaviours, intimacy and exposure. As in the case of Akner-Koler's aesthetic laborations [Akner-Koler and Ranjbar, 2016], sketching ideas over a body shape is helpful.

\section{Capturing body information. EMG, ECG and EDA}

Electrophysiology acquisition platforms aim to characterise body changes via fluctuations in electrical properties. One of the first obstacles that physiology systems encounter is that of noise.

\section{Noise}

Signals that need sticking electrodes on the body, are very much dependent on the + - electrode placement, as well as the ground reference electrode in an electrically neutral area when needed (e.g. on the skin surface close to any bone). Exploring the effects of noise is highly recommended for the different sensors that need electrodes attached to the body.

Before properly exploring any signal that uses electrodes, feel free to start the acquisition and give a look at the information that is present. Proceed to connect the + and - electrodes. Observe how the signals change when the ground electrode is put in place. Can you misplace the electrodes? Is the space between electrodes changing how the signal is represented? Test different locations and see how signals are modified. 


\section{Exploring Electromyography (EMG)}

Describe the setup: EMG Electrodes are placed on the surface of the muscle that we want to monitor. +/- electrodes must be close to each other, preferably along a muscular fibre. The ground electrode used as a reference must be placed on the body, in an electrically neutral area (e.g. a bone where no muscular activity is present).

Preparation: Close your eyes. Take a deep breath. Leave your arms hanging freely, and extend your arms in front of yourself. Open your hands and hold them facing up, keeping the extended arms position. Can you feel the muscles that are involved in holding this position? Try turning your hands now, moving them to face the floor. Can you feel how other muscles are important for this position?

\section{STEP BY STEP EXAMPLE}

Let the participants leave their hands hanging. For instance, rest the wrist on a fixed surface (or on the own knees) so that the arm is completely relaxed. Place the electrodes to the arm (forearm, biceps, triceps) and the ground to the bone. The raw EMG should be of low energy, exhibiting small amplitudes on the graphs.

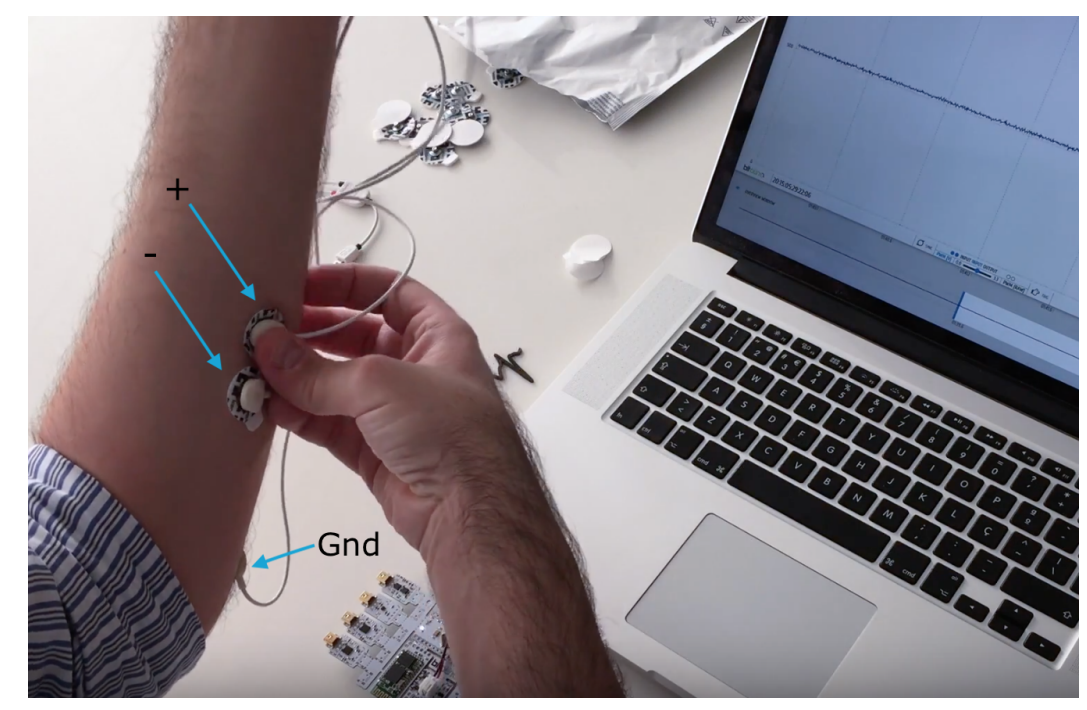

Figure 4.6: BITalino EMG setup

There should be a constant rhythm of peaks along the timeline. You will need a heavy object that can be caught fairly easily, such as a bottle of water of 
$0.5 \mathrm{l}$ - 1.0l. Have someone else hold the object above the participants hand. Start with a small distance and increase it gradually. Drop the object onto the open hand, allowing the participant to catch it. Mark the time before and after the drop, and the point of the catch. The EMG should rise just before catching, and then spike rapidly once the object comes into contact. If the users close their eyes, how does this affect the signal? Is the attention to the visual feedback altering how one clenches a fist or activates certain muscles?

Exploration: EMG signals highlight how we are/are not in control of muscular activity. Being relaxed, try to discover how to activate a particular muscle. We do not actually know how to activate or relax several muscles. Follow this exploration and notice how rotation and flexion change the fibres involved in the muscular contraction. We are looking for noisy signals that show high amplitudes which contrast with flat resting signals.

Try to tell muscles apart. Test some complementary muscles. Which are the muscles responsible for the different fingers (several EMG sensors)? Fibre direction is crucial, in order to understand how signal propagates. Place electrodes perpendicular to fibres and notice that it does not make sense. Generate a situation to make the muscles shake (abdominals and plank exercises are very subject to this). Accelerometry (ACC) sensors are great complements to add to this experience. Keep a physically demanding position (e.g. planking position on the floor) for some time while measuring key muscles in the abdomen or arms. An interesting insight can be provided by comparing symmetrical EMG measurements and assessing the presence of different tensions (e.g. due to handwriting, dominant hand holding weights more often, etc.). Testing different gestures like pointing, grabbing, raising a hand could be relevant to highlight the role of the different muscles. Consider using light weights to test how muscles react to "catching" and/or "holding" in different conditions. What happens with weight and pressure? Hold some weights (and keep adding) for a while to experience some changes. Feel the shaking. Provide a physiological explanation of the phenomenon measured: Describe what EMG is. When you perform a movement, or when you hold a position, muscular fibres receive electrical information in order to tense. Muscular activity is revealed by paying attention to such signals measured along the fibres. 


\section{Exploring Electrocardiography (ECG)}

Describe the setup: Choose an electrode placement that is suitable for your Soma Sensing experience. There are several electrode placement options that you may want to explore (e.g. ECG electrodes placed on the chest or on the hands).

Preparation: Close your eyes. Can you feel your pulse? Is the blood being pumped to your limbs noticeable in any way. Where is it most remarkably present? Exploration: Is ECG a signal that we can control as we please? How can we disturb the signal? Try to change your breathing so that your pulse is modified. Does the rhythm change when changing position? Can you try a physically demanding gesture/position and assess how this affects your heartbeat?

Are you able to observe how the respiration outer envelope encloses the ECG peaks? Try to slow it down by slowing your respiration. Sustain respiration and see the changes in heart rate. Try the Valsalva maneuver, which can be used to explore effects in ECG. The Valsalva maneuver consists in strongly breathing through the mouth while the nose is held tightly closed, creating a pressure that impacts the heart rhythm. Is there a way to introduce noise in the acquisition? Try moving, walking, pulling cables and electrodes.

Provide a physiological explanation of the phenomenon measured: Describe what ECG is. ECG measures the electrical activity of the heartbeats. Electrical impulses responsible for the polarisation of the heart are generated with every beat. By placing electrodes in specific locations, voltage drops can be tracked, showing the heartbeat electrical signals and highlighting the different standardised ECG waves.

\section{STEP BY STEP EXAMPLE}

Place the electrodes onto the participants' chest and monitor the signal as they are standing/sitting still and breathing normally. 


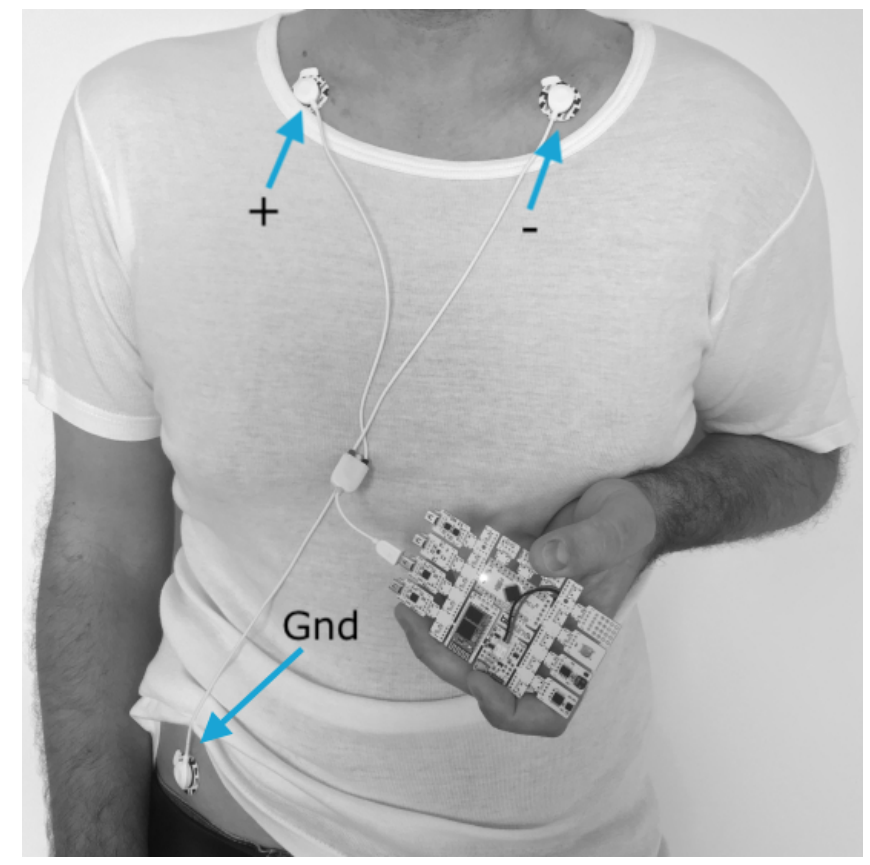

Figure 4.7: BITalino ECG setup

There should be a constant rhythm of peaks along the timeline.

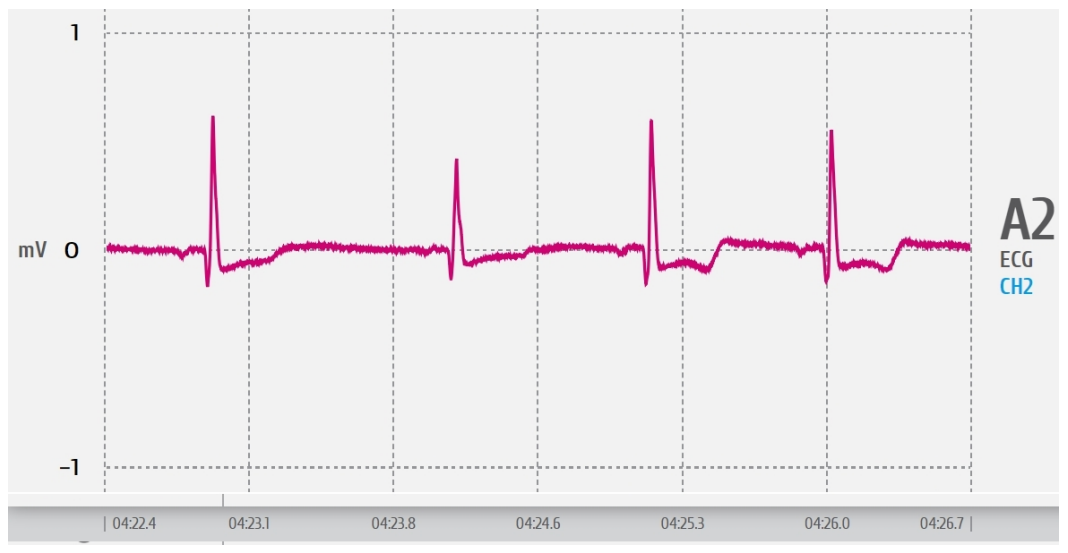

Figure 4.8: ECG signal representation

Follow the user through a mindfulness exercise and record a baseline signal. Address how the participant reacts:

1. Record the signal as the participants rapidly jump around until they can 
feel a change in the intensity of their heartbeat.

2. Ask the user to spread her/his arms in and out from their chest, staying conscious of their own breath. Record the signal as the participant moves slowly between these states.

How do the above exercises affect the signal? Do you still recognise the peaks? If so, take note of their periodicity and amplitude.

\section{Exploring Electrodermal Activity (EDA)}

Describe the setup: Electrodes are placed onto palms or fingers. Sole (foot) is also a possibility. EDA is a slowly varying signal, much better observed when working with longer time-scales (slow variations, that take time to appear).

Preparation: Close your eyes. Take a deep breath. Leave your arms hanging freely. Pay attention to your hands. You can move the fingers slightly if you want. Are you able to feel the room temperature with them? Is there any perceptible airflow? Bring the participant closer to her/his body sensory awareness.

\section{STEP BY STEP EXAMPLE}

Keep the ECG sensors attached if you have used them previously. Place a set of EDA electrodes onto the fingers or palm, separated by a small distance.

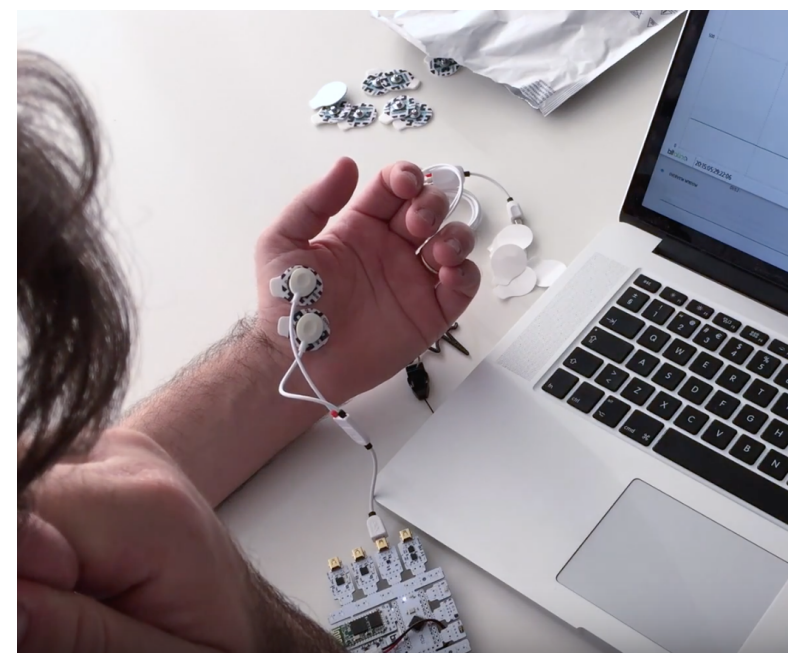

Figure 4.9: BITalino EDA setup 
Allow the participant to follow a mindfulness exercise. Guide them towards a relaxed state. They should be comfortably seated and able to breathe fully. Zoom into the signal, is there much change? Stimulate the user(e.g. show scary video). Record both signals as they are watching the video. How does the EDA signal change when the user is aroused? How does this signal compare to the ECG signal in regard to time.

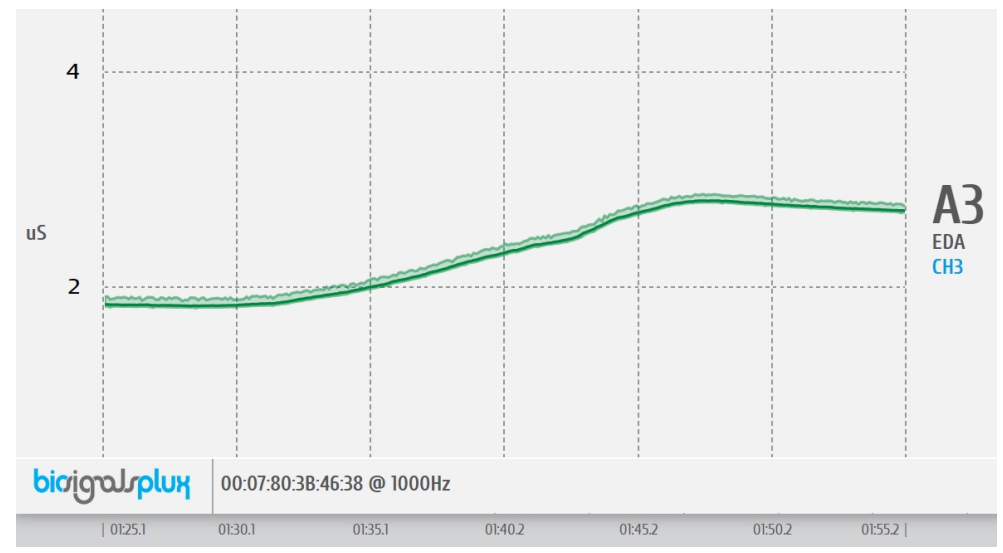

Figure 4.10: EDA signal representation

These are examples intended to help the interaction designer that wants to integrate biosensing into the design of experiences grounded on the body.

\section{Exploring Electroencephalography(EEG)}

Close and open your eyes.

Alpha signals are present.

Carry out a cognitive task.

Try meditating.

Explore different placements.

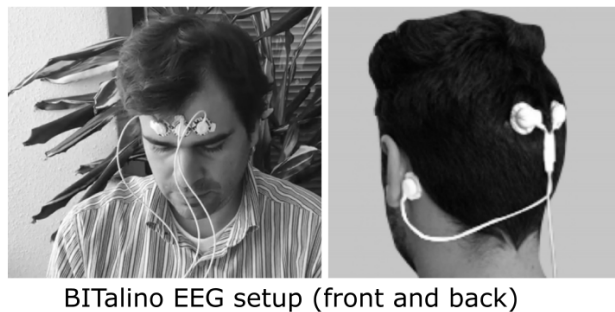

Exploring Breathing sensing

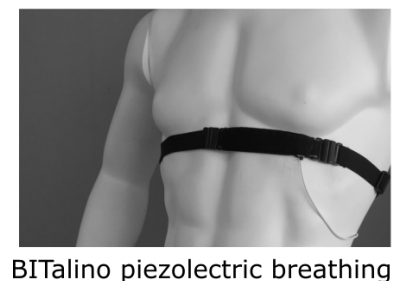

Do chest and abdominal signals differ?

What about different people?

How does coughing appear in the signal?

Hold your breath. Breathe in and out.

Can you spot changes in (rapid/slow) flow?

Figure 4.11: Creating extra examples of first-person sensing (EEG and breathing) 
The user should be able to create their own and go beyond, e.g. with electroencephalography or breathing sensing.

With them, and with the support of biosignal acquisition tutorial materials found around low-cost platforms (see section 2.1.1, section 3.1) we aimed at setting the ground for a growing body of research into first-person sensing, leveraging the role of the body not only as an input mechanism but as a main player to make sense of the experienced data and sensations that one can attach to it.

\subsection{From Biodata to Somadata}

Biosignals are time representations of changes in energy produced in the body [Semmlow and Griffel, 2014].These changes correspond to energy variations of different nature such as in electrical, chemical, mechanical and thermal processes (see section 2.1). While affordable costs motivated the proliferation of biodatabased applications in everyday life [BITalino, 2013, Grove - Seeed Studio, 2014] the design of biosignal-based interactions beyond biomedical paradigms still remains much unexplored. When designing with biosignals, several aspects come into play. Biodata comes in many forms. This research mainly draws upon psychophysiology-based and movement biosignals. However, data of other nature, for instance behavioural, routine-based, on reproductive health or related to the excretory system, could also be considered for the design of novel biodatabased interactions. As such, there is room in interaction design to explore the engagement with data describing drug intake, sleep cycles and circadian rhythms, nutrition / food consumption, menstrual/reproductive cycles, physical activity, etc. The input modalities have an enormous impact on the biodata interactions that one can create. When it comes to affective technologies, for instance picking arousal as an example (see Sanches et al.'s [Sanches et al., 2010] biofeedback stress study), it is possible to address heart rate variability fluctuations, capture blood pressure or measure changes in electrodermal activity instead. Every source of data presents characteristic affordances. Moreover, even if a source of biodata is chosen, several sensing mechanisms with their advantages, their different fidelity, form factors and drawbacks exist. Different sensing modalities enable different design directions, with their own consequences and constraints. For this reason, not only choosing the input source is important, but exploring several sensing mechanisms benefits interaction design efforts aiming to approach biodata as a 
design material. Some researchers that are found at the intersection of the arts and the design of technology-enhanced interactions, such as Fdili Alaoui [Fdili Alaoui, 2019] call for embracing technology resistance and turning it into artistic creativity. Sensors often accept many placements. Hence, design research should consider different body locations and how every choice conditions the quality of the data and the resulting interaction. Biodata is in many cases immaterial. As such, design insight is not easily provided by the technical specifications of the sensor. When raw data is recorded, processing mechanisms to distil the information meaningful for the designed artefact or interaction need to be in place. For instance, an affective tracking system monitoring face expression to infer emotion aspects, may work via facial electromyography, hence needing the processing capabilities to precisely detect muscle contractions at the relevant face spots and assign affect meaning to it. Alternatively, systems based on cameras, hormonal tracking, heart rate or electrodermal activity with the proper processing mechanisms may either corroborate or contradict the inferences. Every sensing system needs processing mechanisms to manipulate data. Sensing multimodality, becoming more and more common, adds robustness at the cost of increasing the complexity of the monitoring experience (more sensors, more cables, more electrodes, and different processing paradigms working concurrently).

\subsubsection{Biosignals as a design material}

Echoing the work of Solsona [Solsona Belenguer et al., 2012] and colleagues on immaterial materials, interaction design should strive to find different ways to explore the affordances of biodata as a design material. As already stated, biodata is in many cases immaterial. Could alternative ways to design interactions, grounded on material exploration and the experience of the sentient body, help embrace biodata and its meaning-making process? If one thinks of technology that has already made it into everyday life, systems for physical activity tracking, cardiac health or sleep quality stand out. These systems usually capture and provide data in a concrete way, i.e. via a screen display, through charts, tables and text, with a given choice on colours, shapes, and dynamics. Recent $\mathrm{HCl}$ research [Howell et al., 2018a, Merrill et al., 2019] has found that when dealing with biodata - in general data of medical nature -, the layperson ascribes precision and authority to the data, or even to the platform. Sanches et al. [Sanches et al., 
2019a] explain how user groups come up with completely different interpretations around an EDA-based monitoring system. The interpretations of biodata are inevitably tainted by the user background, to the extent that, sometimes, the expectations of what is to be seen in the data rule out any possibility of accepting meanings that go against them. Not even when the data supports such meanings. Data interpretation and meaning making are crucial for the design of affective technologies and interactions enabled by biodata, as for instance mentioned in section 4.4 and the work of Hollis et al. [Hollis et al., 2017]. But users do not necessarily possess the data analysis skills of a trained data scientist, nor technology should expect it from them. When designing biodata interactions, one can take different approaches, i.e. focusing on health diagnosis, aiming at the detection of patterns or specific events or, in the case of exploratory designs adopting an open-ended attitude that moves away from engineer-oriented uses. The approach of the interaction designer is not necessarily a solutionist one, i.e. an effort looking for the best solution to a given problem. Rather, interaction designers are used to address ill-defined problems [Cross, 1992] and leverage material exploration to elucidate different ways to address such problems. As explained in section 4.4.1 and Umair et al.'s works [Umair et al., 2018, Umair et al., 2019b], not long ago, Human-Computer Interaction research experienced a turn towards the material [Fernaeus and Sundström, 2012, Wiberg, 2013]. The idea is that through materiality, design ingredients that are difficult to grasp - as it is the case of biodata - can be transformed into something can be touched, tangibly understood, modified, tuned or programmed [Vallgårda et al., 2017]. Biodata could be seen as a material to design with, with its affordances and aesthetics [Elblaus et al., 2015]. As exemplified with the ThermoPixels' thermochromic material exploration, touching, feeling and establishing a conversation with the materials leads to an analysis of possibilities, and potentially novel ideas. Explorations of biosensing data as material are still a small part of $\mathrm{HCl}$ biodata studies. Our research, borrowing ideas from the affective technology design field we aim to contribute to, and armed with the help of a first-person soma design approach, is set to work in bridging that gap. 


\subsubsection{Collaborative design of biodata interactions}

The insights presented here are the result of a design journey that was born with the great inspiration that a training on first-person design methods brought (see section 4.2.2 and 4.7). Highly motivated by the somaesthetics of actuation haptic explorations that followed Akner-Koler's [Akner-Koler and Ranjbar, 2016] sensitising lab concepts (section 4.2.4) and with ideas on how to translate biosensing into a sensing self experience (see section 4.7) we set out to explore ways to turn biosensing into a design material. This design journey took the form of 4 collaborations in the context of project AffecTech [AffecTech, 2017] meetings, training events and organised workshops that took place at different universities across Europe. From the beginning to the end, soma design (section 4.2.3) was kept as the background design approach, with a strong focus on the sentient body, disrupting the habitual and designing slowly. By disrupting the habitual, in a body-centric interaction design exercise, we are referring to alternative ways to move or interact with our own bodies and surroundings, using strategies that allow breaking body actions into steps so that one can rethink what is involved in them. The purpose is to either analyse or gain awareness of body aspects - suggesting an arm folding gesture in the opposite way as usually performed is an example we often use to convey such disruption ideas. The AffecTech collaborations - or design stages - that built our study benefited from numerous participants from the EU project, but also from designers and users that engaged in biosensing explorations across institutions. By organising soma design workshops, our research progressed, took learnt lessons from design stage to design stage, eventually culminating on the successful technology couplings and interactions reported here. However, these are fruit of an open-ended exploration, where insightful interactions were far from anticipated. After reflection on the A-lab possibilities (see section 4.2.4) for sensing and having written about the sensing self ideas (section 4.7) we put them in practice by means of 2 group design sessions conducted at KTH Stockholm. For the design sessions, the BITalino [BITalino, 2013] biosignal acquisition platform was facilitated, offering the tracking of different physiology signals. Our exploration highlighted the interest in engaging with biodata bodily, rather than mere visual representations that are abstract or disembodied. As in the case of the A-lab, a heightened sensibility for biodata and sensing was sought. An important design experience improvement came when, asked by other interaction research 
colleagues, we were asked to prepare soma design sessions that touch upon the topic of balance. These were to take place in Nottingham's Mixed Reality lab, in order to explore a collaboration combining soma design and sensory misalignment (see section 4.6). The relevant step forward consisted in addressing the design of an interaction as a challenge to touch upon the balance topic - working in an open-ended way but striving to put technology together to either achieve evocative effects or novel ways to disrupt (or misalign) experiences. One such way of putting technology together was a coupling of biosensing with generated biofeedback audio cues deployed during a balance activity in which participants had to cross over a beam. The novel interaction insight that couplings brought was amazing. With the same enthusiasm, another topic, synchrony, was picked for a similar group soma design workshop that took place in the Cattolica di Milano University. In that occasion we set out to continue exploring the somaesthetics of biosensing-actuation couplings.

\subsubsection{Biosensing-Actuation couplings}

The main takeaway from the soma design sessions is the concept of somaesthetic biosensing-actuation couplings. Throughout our design research process many possibilities were investigated, but only some successfully provided design insight. These are the ones described in this section.
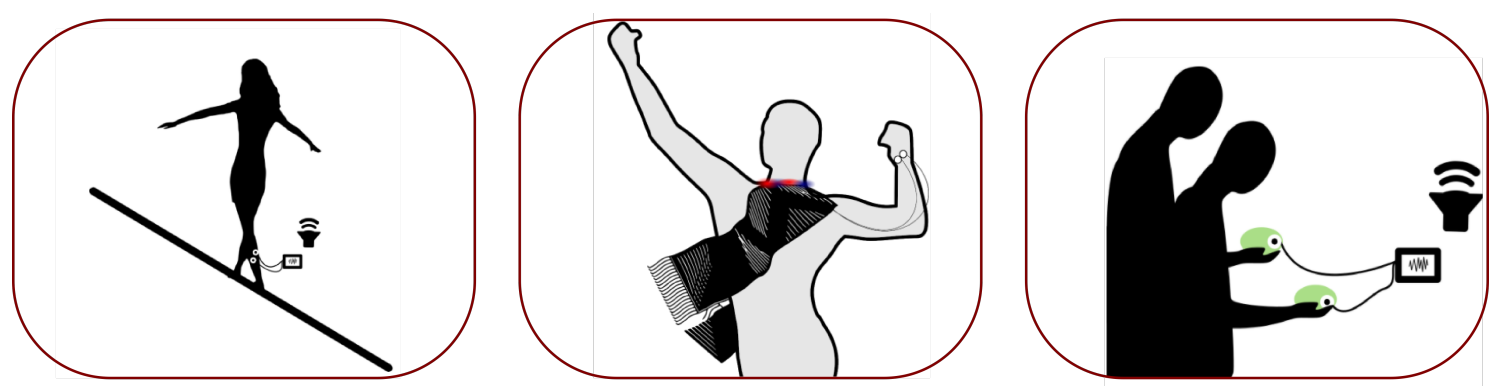

Figure 4.12: Somadata coupling examples: Balance EMG-to-sound experience, Scarfy thermal-based EDA feedback, ACC-based sounds of synchronous movements (left to right)

What we call a successful coupling, is a coupling that is grounded in what the biosensing and biodata afford. Moreover, the interaction must respect the design topic through which it was conceptualised. Couplings need to connect with 
our body, with our somaesthetics, and let us extend from it either through firstperson experience reflections or sharing instances. A description of the design discoveries brought by the couplings, which converted them in candidates for somadata exemplars, is provided here.

- EMG Balance premonition: Electromyography (EMG) is the measuring of muscle activity. The estimation of muscle contractions via the inspection of EMG graphs is not always an easy task, dealing with rapidly changing fluctuations and often subtle amplitude differences. By leveraging the use of sound biofeedback, i.e. by connecting EMG amplitudes to sound properties such as frequency, a user is able to receive muscular balance information while balancing on a beam. Moreover, the heightened awareness of the muscle activity is instantly shared with peers who, when looking at real-time EMG graphs of the monitoring platform, can anticipate the loss of balance. A sound feedback like the one presented here, and as opposed to screenbased graphs, does not disconnect the perception of the user's own leg from the balance activity and its first-person experience.

- Scarfy, an EDA Breeze Around Your Neck: Electrodermal activity (EDA) is a signal that is linked to physiological arousal. The challenge to design for synchrony and the renewed interest in crafting couplings that connect biodata to the body brought the focus to ways in which the elusive EDA data could be leveraged. $\mathrm{HCl}$ research has been contributing to the idea that arousal information monitored via EDA can be experimented on the body through alternative visual and haptic feedback mechanisms [Howell et al., 2018a, Umair et al., 2018, Umair et al., 2019b, Umair et al., 2020]. Would it not be an interesting design aspect that such an awareness could be synchronised between two people? That would mean that arousal states count with measurements synchronously triggering feedback mechanisms that the subject herself/himself can experience on the body. If the feedback is deployed in the form of a wearable, arousal effects become shareable. That is how the concept of the Scarfy, i.e. the temperature haptic EDA scarf was born. Interestingly enough, the Peltier elements used for creating the coupling exhibit a peculiar quality. While heat builds up on a side when subject to electric current coming from the EDA control mechanism, the other side of the thermoelectric cooler decreases temperature. During the soma 
design sessions, where the body was used as the main source of experience, material, embodied explorations emerged naturally. When participants realised the dual properties of Peltier elements, they felt prompted to try and turn elements upside down. Eventually, what this created was the notion that beyond cool-warm effects achievable by the Scarfy, EDA data is malleable, shapeable and re-interpretable.

- Sounds of Synchronous Movements: When designing for synchrony, the idea of capturing movement emerged naturally, as synchrony is per se a monitoring challenge when tracking several sources of movement simultaneously. At some point, the exploration of accelerometers (ACC), used in this case to sense changes of speed in the form of stops, turns, shaking and abrupt bumps, led to the implementation of an experience involving two different hand-held ACC sensors. Quite remarkably, following the inspiration that the audio EMG balance coupling had brought, a simple algorithm fine-tuning the pitch of a chord with respect to differences in acceleration measured in two different spots was implemented. When tried out without instructions, participants hearing the output audio felt encouraged to bodily explore how the device worked. As it often happens in contact improvisation, a non-verbal communication set in, yielding a playful attitude that led to choreographies, gestures and actions that were aimed at understanding the technology. More importantly, participants went beyond and realised the limitations of the sensing, only operating in one axis (either horizontally, vertically or back-and-forth), hence challenging the interpretation of data, turning one sensor upside down and appropriating the accelerometry data and its meaning-making process.

\subsubsection{Somadata and design insights}

Quite remarkably, at the end of this design journey, we had arrived, by means of couplings, at the definition of a new concept: Somadata. Somadata is the experience of biodata from a firs-person somaesthetic perspective. Somadata experiences are collaborative, supporting collective meaning-making and the sharing of design insight. Somadata should not constrain the exploration of the body dynamics in the chosen design context or topic. The couplings' design insights that made us recognise them as somadata are three. Firstly, through our 
soma design couplings biodata becomes a design material. This rings true for the EMG, EDA and ACC biosignals related here. Although not highlighted more emphatically, this success is the fruit of the thoughtful preparations of the materials before each workshop took place. Data from biosensors, rather than being "raw", becomes in fact a carefully crafted sociodigital material, readily to be experienced and molded by workshop participants. Here, sociodigital material [Höök, 2018] refers to the combination of the digital materials and our social and living bodies that we design with and for -also subject to change via the interactions we perform. Secondly, the concept of couplings is stretched further to play a relevant role in the creation of interactive experiences - not only "cool" feedback effects. As such, interactive experiences need to be orchestrated. Coupling mechanisms count on components that need to be turned on at the right time. Control of dynamics over actuation-deactuation cycles must be sought. Furthermore, as soma design continuously reminds us - and of relevance for affective technologies - every subjective felt experience is different. Hence, orchestration mechanisms must also be put in place so that re-programming, re-purposing and customising the technology couplings is a reality. These are insights that pave the way for future interaction research where similar bodily sensing-actuation paradigms are of interest. Finally, the third aspect of somadata is their shareability, highlighted in the 3 examples that were developed throughout this study. EMG signal novelty lies, partially, in the fact that not only a balance anticipation is achieved through sound, but it is also made available to colleague participants that can be part of a collective meaning-making experience. Scarfy is born with the desire to share arousal effects. The approach is appropriated by participants, and EDA data mappings are challenged and enriched. In the case of synchronous movements, audio-based ACC data is shared through movement coordination and several gestures. All of these are examples that could guide $\mathrm{HCl}$ research already addressing the sharing of biosignals [Howell et al., 2019, Liu et al., 2019], now with a strong focus on the lived body.

\subsubsection{Limitations}

Through a range of soma design-inspired workshops we explored how to bring forth interesting qualities in biodata to motivate designerly imaginations. Successful explorations are what emerged from engaging with what we call somadata - 
that is turning the biodata into a "tangible" form by coupling it to sensuous actuation, enabling first-person, felt and shareable encounters that are aligned with a design context. However, even if we provide the ingredients that were part of our design path, i.e. the strong soma design focus, the sensitising methods, the multimodal sensing platform, etc. there is no way to present this design approach as an infallible one-size-fits-all technique. In fact, questions would arise even if presented as a technique in itself, as work between workshops was strongly conditioned by a choice of topics that does not generalise well - a choice that was motivated by researchers' backgrounds and evocative properties we place on design context. Besides, we believe that the balance workshop worked very well because of the $\mathrm{HCl}$ expertise of the participants, the long and quietly paced timeline adopted, among others. However, we currently have no absolute certainty in that regard, perhaps calling for further meta-design research on the standardisation of workshop structure, materials and activities. Although these couplings are examples that we found successful, many others did not make it into a final prototype that resonated with our somaesthetic appreciation, e.g. a movement tracking to liquid thermal/weight exchange actuation, a breathing sensing to vibration feedback, etc. While these have become challenging projects that are still deemed work-in-progress we have no mechanism to guarantee that they will evolve towards somadata artefacts at some point. Hence, somadata should be looked as a collection of exemplars developed with a strong theoretical soma design backdrop behind and depicting how biodata can possibly be turned into design material, potentially inspiring research in similar directions.

The chosen couplings led to interesting design discoveries that, from the users'/designers' point of view, talked to their own somatic experiences. This took place via shared interpretations within design groups, shedding light on how to craft interactive experiences with biodata in a way that is available, and that stems from a holistic soma view. 


\section{Chapter 5}

\section{Making it real. Discussion on biosignal-enhanced body interactions}

\subsection{A research outlook on biosignal-enhanced in- teractions}

Throughout this PhD research journey, the contact with researchers in biomedical engineering and the programming of biosignal processing algorithms has contributed to grasp directions in which biosignals can be further processed to offer elaborate real-time features beyond raw data (chapter 3). In turn, the contact with researchers in the fields of human-computer interaction and design has undoubtedly contributed to building an understanding of what the creation of biosignal-based experiences entails (chapter 4). The strategy of attending to the users' needs - or the users/designers themselves - through first-person soma design sessions, and DIY crafting practices in the case of arousal representation kits, has proved to be a rich procedure. The research started from a science or engineering perspective that studied how biosignals can be made available for the developers who want to benefit from them in terms of improved information processing (health and sports). It is through the shared design insight that emerged from design sessions that an understanding of how to make biosignals available for a more general user was gained. But the main achievement is the approach 
to understand novel ways to leverage biosignals in interactions. Only through interdisciplinary dialogue (even if oftentimes non-verbal) has this understanding been formed. In an attempt to look at the overall research, aspects borrowed from the two perspectives are brought forth. Teaching resources and interfaces (see [Tsaknaki et al., 2019], section 3.1) help support the interdisciplinary dialogue, of course. But only when the opinions of the engineer clash with those of the interaction designer is one forced to seek novel alternatives to understand biosignals as a material. This perhaps echoes a too much compartmentalised science doctrine, be it on the engineering perspective or the interaction design one - or both. The artefacts resulting from this research, or the novel interactive instances in which biosignals are made into tangible and shapeable material (section 4.8), speak in favour of a fruitful way to address biodata differently. In this chapter, joining the biosignal developer and the interaction designer perspectives, a view on open orchestration research avenues is presented. The following are the best candidates to capitalise on both strands of work to make biosignals more available:

- The study of ways to extend the orchestration of biosignal-enhanced interactions by means of refined biosignal processing techniques and interactive machine learning tools

- The extension of the repertoire of somaesthetic biosignal-actuation couplings, adding "bits" to the SomaBits collection [Windlin et al., 2019]

In the following sections, both aspects are described and addressed with examples.

Beyond such research lines, there is also potential for the field to progress in two other directions that have not been sufficiently explored in the context of this thesis. On the one hand, research can further pursue what could be dubbed as "Dare to design". The successful encounter of two design studios presented in section 4.6 highlights the benefits of committing to long, slow-paced body interaction design exercises which brought relevant insight. With biosignals that were an added ingredient to leverage body inputs, the experience grew from a meeting that ended up in prototype concepts that succeeded in defamiliarising a design experience [Bell et al., 2005]. However, there is room to further unpack the dimensions that are navigated when designing together (e.g. levels of comfort, body boundaries and familiarity or skill). This could be done, over time, with in-depth analysis of the 
gathered materials used within the organised workshop [Tennent et al., 2020], but also conducting new sessions involving other design teams or background topics. The described experiences exploited the significant expertise in entertainment and misalignment strategies that our study collaborators counted on, but the same design strategy could be in principle applicable to other interests and design labs. Just as a first-person approach pushed researchers to dare to try themselves [Umair et al., 2019a] and go through discomfort, limits are still to be set in how far one would go in trying technology within the body, where aspects such as body contact and awkwardness are at play. On the other hand, if one pays attention to the outcomes achieved in [Alfaras et al., 2020b] or even the structured study shown in [Umair et al., 2020], it is worth to acknowledge that no test was done on the long-term usage of somadata prototypes exposing body aspects nor affective arousal gadgets exposing emotionally-charged and personalised representations.

\subsection{Orchestration, design platforms and interfaces}

At the end of the bodily-inspired sensing-actuation research journey (section 4.8), one of the main, unveiled aspects of relevance for body interactions is that of orchestration. Orchestration refers to the ways, timings and effects of an interaction that must unfold according to the measured biosignals and features. Orchestration links body measurements to graspable outputs or feedbacks that act on our body in a way or another. The effects that take place must be programmed, sequenced and organised, even allowing a configuration by the user/designer so that actions are customised to her/his needs or preferences. These either take the form of programmed (software) actions such as sound generation or effects that take place, e.g. when a muscle is activated, or wired (hardware) sequences such as thermal based actuation connected to electrodermal activity increases. Moreover, the orchestration should not be seen as a mere immutable strategy that has been decided upon by a designer. Ideally, researchers and designers should count on interfaces that, if desired, can expose the subjacent mechanisms of the technology to be understood, improved and confronted. During the soma design workshops or sessions in which participants experiment in groups, the orchestration mechanisms prepared should be facilitators of the technology exploration. Using the felt body with an evaluative stance, the exploration of biosignals and actuation mechanisms relevant to self-awareness (body and emotional) has to be enabled. The designer 
needs to break down interaction steps and either adopt or craft new meanings and interpretations. The designed systems, either seeking body or affective selfawareness objectives, need ways in which body input-output and meanings are put in place, coupled, coordinated, customised, sequenced and exposed so that the underlying mechanisms can be better understood, challenged or extended. Experiences that are tangible, i.e. that "touch" the user, somaesthetically, must be sketched, thought and programmed, from the hardware and software point of view. An orchestration is needed, serving several (general) purposes:

- Technology components must be able to communicate information reliably

- Interaction modules must be able to process information, without compromising time

- Information must be represented, triggering coupled effects in line with the designed experience, exposing elaborate information beyond mere raw data

It is crucial that inputs (biosensors) and outputs (actuators) are nicely coordinated. Orchestration mechanisms as described here aim to facilitate the exploration of biosensor data and meaningful representations through actuation that addresses modalities beyond visual feedback (see section 4.5, [Devendorf et al., 2016] on wearing screens, and [Umair et al., 2019a, Umair et al., 2018, Alfaras et al., 2020a] on alternatives). For this to happen, several aspects are identified:

- Coupling modalities: (New) Couplings need to be in place, i.e. biosensors and novel body output effects (actuation/feedback) need to be connected. These require data acquisition and processing capabilities, as well as the ability to provide responses. Ideally, couplings should be able to be made, modified and turned on or off

- Sequencing/coordination: Input/output modalities need to be sequenced. Effects must follow an order, or count on event-based rules that govern which effect goes first

- Design platform: A design platform, or an ecosystem of devices, must be able to guide users in understanding the captured biodata through their felt experiences. This design platform must provide the design exploration ground, showing capabilities, limitations and roles of the involved technologies that participate in the interactions 
The work of Windlin et al. [Windlin et al., 2019] on the SomaBits is a precursor of these aspects, which has been - and will continue to be - inspirational for the research on coupling biosensors to body actuation. Strategies and effects will grow as body input (sensing) and output modalities (e.g. actuation mechanisms) increase [Alfaras et al., 2020a]. Through physical artefacts that make up a design toolkit, design session participants or users are invited to explore, try on and test effects on themselves. The spontaneous material exploration observed in experiences such as the ThermoPixels section 4.4, where participants use their own bodies to understand how thermal effects and thermochromic changes work, is somewhat outlined. In the case of First-person Physiology (section 4.7) despite working as an exploratory framework, the session's structure criticism received mainly from $\mathrm{HCl}$ scholars participating in design workshops, is somewhat diluted when working with more elaborate exemplars, such as those encountered in [Alfaras et al., 2020b]. This has to do with the appropriation of the meaning-making process and biodata involved - a process observed with arousal data too in the ThermoPixels study due to the crafting focus-, arguably resulting from the firstperson perspective and particularly strengthened in the case of participants who attended design sessions repeatedly.

\subsubsection{A repertoire of biosignal effects}

In the creation of a Soma Design toolkit, we aim at achieving a wide range of customisable modalities for the user to explore possibilities that relate to her/his body. In this thesis, and in particular in the study addressed in section 4.8, the takeaways should revolve on a path that proved successful to connect or engage with biodata differently, in a material and body way. This is exemplified by coupling exemplars. However, by no means these are presented as the sole and ultimate couplings, generalisable to anyone. What is unpacked in [Alfaras et al., 2020b] is a way to address biodata, to be explored having our bodies into account, leading to an appropriation of meaning and technology that one can change and design with. Ultimately, one can create the effects what communicates best for her/his soma. The exemplars are exemplars because of their condition of generating design insight or somadata aspects, i.e. being able to be felt personally, able to be shared and understood differently and collectively and in line (not against) a design context or activity. Through the proposed approach, which is to be understood as a 
suggested strategy rather than an infallible recipe, biodata has been appropriated by interaction design. Echoing principles relevant for soma design, such as that of disrupting the habitual or estrangement [Wilde et al., 2017], it could be argued that biodata has been deconstructed. Somaesthetic first-person design is not the only way to deconstruct biodata. Examples exist in exposing biosignal related data in the public space, to be shared and experienced while capture or re-experienced in future [Howell et al., 2019]. Sound designers have addressed the design of instruments with patterns that represent chemotherapy sessions, in a mixture of body and emotional data that is tangible and can be replayed [Rocchesso et al., 2019]. Artistic projects such as Lozano-Hemmer's Pulse Park [Rafael LozanoHemmer, 2018] for instance explore the shareability of pulse signals from the heart and how they can be interconnected with architectural elements.

Soma Design provided a path for an interdisciplinary design team to embrace this material and open the design space while keeping the researchers grounded on the body - an aspect we consider fundamental for the affective interaction / technology approach. This is what made couplings successful, i.e. giving us design insight such as the playful non-verbal movement arrangements obtained through synchronous accelerometry or the shared audio-muscle activity in the context of balance. The same applies when considering the reinterpretations of electrodermal activity data via heat dissipation felt thanks to a scarf, all of them irrespective of whether an artefact is to be properly evaluated by means of user testing or further developed -which was never the goal of this material exploration to make biosignals available. It is worth stating clearly that the prototyping efforts presented fall short on making a very wide range of possibilities available to the users to experience biodata with their bodies. In line with this, for instance, some of the usual affective computing biosignals have not been successfully approached for the design of interactions covered in this thesis. That is the case of, among others, heart activity (information that counts on established processing mechanisms ), body temperature (an often ignored signal, or simply complementary, that is easy to monitor) and breathing activity, to some extent. In terms of biofeedback modalities, shape-changing actuation, taking physical changes to the tangible, haptic and beyond-the-screen visual domains, has been inspirational throughout the soma + affective design research undertaken but still remains work in progress as per the results achieved by our own biosensing-actuation integration efforts. Technology-wise, $\mathrm{HCl}$ research has already shown some of the design potential 
behind linear actuators and inflatable shape-changing mechanisms that inspire and guide future research perspectives. Examples of these effects are Project Materiable [Tangible Media. MIT Media Lab, 2016, Nakagaki et al., 2016] and Project Lift-Bit [Carlo Ratti Associati, 2016]. These remarks should not be read as if soma design is not suited to explore certain biosignal (input) modalities or biofeedback effects. Beyond the expertise of the designers that this research has counted on, the interplay of body aspects recorded, how they are processed to expose characteristics beyond raw data, the technology limitations or affordances, the body-centred practices surrounding the design sessions and the context in which they unfold have an impact on whether the resulting coupling relates to our bodies. Hence, there is room for improvement if properties or effects that already stir our interests are further studied or, as shown in the misalignment research (section 4.6) one does so by letting discomfort, fast dynamics, thrill, the unpleasant and the unknown enter into the design exploration to enrich the soma perspective.

\subsubsection{Machine learning for biosignal interactions}

As seen in the first chapters of this thesis (chapter 3), a relevant part of the research presented here has focused on the extension of biosignal feature extraction, processing, and real-time analysis (e.g. to potentially enrich real-time feedback possibilities within the lab and in more ecological settings). The discussed couplings (e.g., as presented in section 4.8) make use of basic signal processing. Further possibilities, however, lie in improving the current algorithms and processing approaches. Any sort of biosignal acquisition, in particular in psychophysiology, can be seen as the process of dealing with sequential and timeseries data. Prior to the deployment of feature extraction and selection techniques, data must be preprocessed properly. ECG, EMG, EDA, IMU, respiration, and all the data collected from available sensors are in general sampled at different rates and present different properties that entail signal noise and instances of data that do not conform to (standard) expected representations. These can be seen as artefacts or "meaningless" data. Since the data obtained in real-world ambulatory settings is always prone to noise, presenting inconsistencies or missing values, preprocessing and cleaning is required. Depending on the signal, researchers count on specific signal characteristics (section 2.1.2), time domain and statistical features that are suited to real-time processing (see section 3.5). An example is 
seen in the case of heart activity, where mean value of the heart rate, the mean value of the inter-beat intervals, the standard deviation of the interbeat interval and the root mean square of successive interval differences are accessible in real-time, in the wild (see section 3.5.1 [Ramos et al., 2018]). Of particular interest are the less apparent frequency-based features which given the demanding spectral analysis computational requirements have just begun to appear in the nowadays more capable out-of-the lab devices. More often than not, it may be interesting to address several biosignal input modalities if unveiling particular regimes or phenomena is what the researcher is after. This is the case of fatigue and the elaborate combination of EMG and ECG features (see section 3.5.2 [Ramos et al., 2020]), but could be applicable to contexts closer to psychophysiology and mental health therapy that would benefit from a multimodular biosignal acquisition offering a broader picture of e.g. stress, emotion regulation strategies, etc. At the same time, multimodularity improves accuracy, allowing to rule out instances of false detection of events more easily. Input output links need to rely on processing capabilities, event detection, and high-level feature extraction to overcome the limitations of too basic couplings that only put in place simple (signal) amplitudeto-intensity mappings. An understudied aspect in this research, is the possibility to address machine learning interactively. This research receives the name of Interactive Machine Learning. The interest in working toward the deployment and refinement of real-time machine learning processing of information is stressed throughout the thesis. However, the interactive aspect is of relevance, not only when exploring preprogrammed effects in multi-user experiences, but also when deliberately working to alter effects and come up with novel biodata-actuation mappings. This echoes early research achievements such as Wekinator [Fiebrink, 2011] and puts forth recent progress such as that exhibited by the Teachable Machine [Carney et al., 2020]. Interactive machine learning [Fails and Olsen, 2003], originally conceptualised as a way to correct machine learning classifications on the fly has evolved significantly. By means of tools like Wekinator or Teachable Machine-like paradigms, it is possible to define actions, classes of images, movements or gestures and link them to a classification output effect. While these are mainly visual- and movement-based, one can hypothesise on richer inputs, i.e. designers teaching specific biosignal-actuation mappings to algorithms. 


\subsubsection{Supporting the design process}

Building on the relevance of orchestration, the coupling concept is paving the way for future research exploring sensing-actuation connections. An orchestration platform supporting biosignal-based interaction design and the available toolkits (e.g. the SomaBits [Windlin et al., 2019]) could be equipped with mechanisms that count on interactive machine learning that would allow to teach physical mappings or couplings on the fly, by doing, rather than deliberately programming.

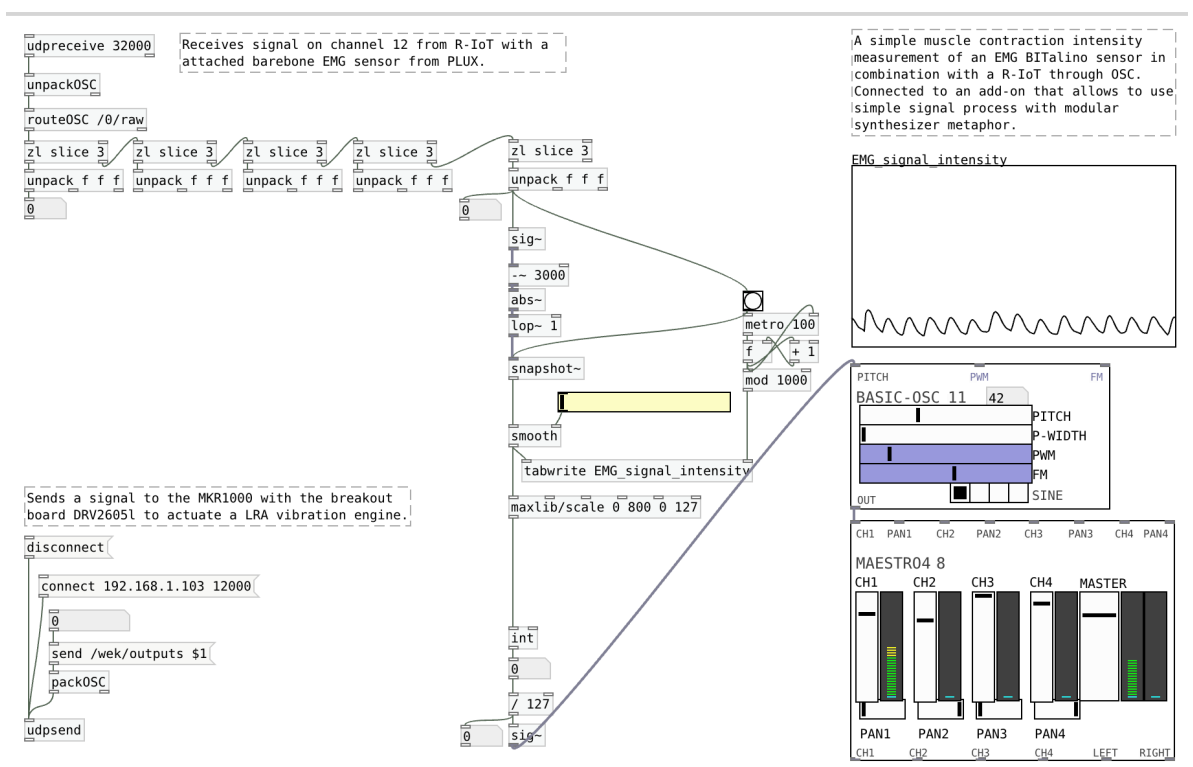

Figure 5.1: Design interface for the creation of am EMG-audio feedback coupling (using PureData).

Hence, the explorative process would move even closer to the body. On the programming side, though, visual programming tools have emerged to connect input and output elements more intuitively, by drag and drop interfaces that enable setting ranges, filtering, value thresholding and concurrent actions. The designer using a modular structure platform where sensors, processing approaches and effects should work Plug and play, needs to access tools to enable both planning (sequencing) and play (exploration, feeling). While this is so far an aspect not centrally studied in this thesis, undoubtedly motivating future research avenues, early experiments exemplify rich possibilities. That is the case of the collaborative work with Windlin himself on muscle activity-sound couplings, feeding into the broader goal pursued at the KTH IxD team on developing affective technology 
design toolkits.

In the depicted example (fig. 5.1), a visual programming user interface where elements are added via drag and drop, PureData (Pd) [Puckette, 1996, PureData, 2020], is used to easily create, link, sequence and fine-tune biosignal to actuation coupling effects, in this case electromyography to sound. It may also be the case that the interfaces support not only the creation of mappings behind the scenes, but its quick modification while exploration takes place. These tools should not be seen as a shortcut to work with biosignals without understanding their affordances. In fact, the act of engaging with them, their features, difficulties, with the presence of noise and nature of their temporal dynamics should be inspired when following a first-person exploration (section 4.7). As Fdili Alaoui mentions with regard to sensing on the skin, technology resistance can be embraced creatively [Fdili Alaoui, 2019]. Here, technology resistance is to be understood as the hurdles and challenges faced when artists approach technologies to be included in a piece (in this case a range of personal tracking sensors used while dancing and the demanding issues of noise, lost/misclassified detections, electrode attachment or operation under intense physical activity regimes). Biosignal-based interaction design platforms bring together the benefits of exposing elaborate biodata (see the teaching materials examples as described in fig. 3.3), elusive features or characteristics as seen in [Ramos et al., 2018, Ramos et al., 2020] (section 3.5) that can, therefore, use effects in order to be made more explicit, graspable, felt or able to be modified by designers. In this process, unique physiology recording and processing technology (section 2.1.1) is leveraged. Ideally, an arranged set of components and preprogrammed effects or coupling modules makes biosignals more available. While multimodular input platforms for biosignals have been identified, e.g. providing a selection of biosignals of interest in affective computing, a mere look at the many coupled effect possibilities (with e.g. changes in tactile, thermal, vibrational, audio, visual, form/shape feedback) indicates that creating an integrative platform for the exploration of actuation is complex. Rather than pursuing an all-purpose body feedback device, it is worth to work on having platforms establishing communication protocols and realtime processing capabilities (or the connection to technology capable of doing it). Besides, it is interesting to count on a modular structure where particular input/output can be inserted at the designer convenience and a defined set of module snippets which make up an effect mapping lexicon that generalises to 
several modalities. For instance, one can think of snippets for the detection and limitation of amplitude ranges, the recognition of specific patterns or trends, the set up of value thresholds, the measurement of between-event intervals (suitable for many electrophysiology inputs) and its connection to output ranges of intensity, duration or periodic repetition cycles. These outputs may require more ad hoc elements, e.g. driving currents for any of the associated motors, transducer degrees of freedom if applicable, as feedback mechanisms differ significantly.

\section{A design interface candidate}

Node-RED is a browser-based visual programming platform for loT development that emerges as a candidate interface to support the design of body and emotional self-awareness interactions linking body inputs and outputs. Through drag-and-drop boxes that users can connect within a main screen, the creation of computer programs (flows) is possible. As such, running (deploying) a flow takes an input, processes it, and provides an output, being able to perform computational operations such as loops and control flow conditionals. The open source internet of things project was born in 2013 at IBM to provide a tool for quickly and intuitively run boilerplate code. Its potential was already foreseen in its early versions by $\mathrm{HCl}$ researcher J. Solsona, who was able to create flows to operate artefacts exposing the immaterial properties of e.g. radio waves [Solsona Belenguer, 2015]. With an ever-growing online community of developers, the platform counts on a wide range of available libraries or extension plugins. Computational functions, called nodes in this case, are editable in JavaScript programming language if needed, but off-the-shelf nodes provide regular input/output functionality and communication with running algorithms, e.g., processing biodata. HTML nodes add a visual user interface screen where clickable elements like buttons, sliders and switches are easily stacked to trigger and modify interaction effects. Just as Soma Bits' actuation knobs and controls enable a rich exploration of haptic effects through the different shapes created [Windlin et al., 2019], Node-RED interfaces enable the fine tuning of input/output mappings on the fly but apparently move away from the interesting tangible approach that Soma Bits offer. The ease of use vs explicitly physical interface tradeoff applies in this comparison. In certain instances, the Soma Bits achieves a remarkable level of simplicity, e.g. by linking an Arduinobased "bit" with thermal feedback that is controlled with our hands. However, when aiming to generalise and couple other inputs or actions, decisions need 
to be made on whether explicitly programming additional components is a good idea. Node-RED features tools to import code from native JavaScript or other programming languages. Working via messages passed from other platforms is feasible. Hence, developing specific nodes to directly launch sensing/actuation commands is a question of trade-offs, since it is also possible to rely on an underlying communication and control hub (orchestrating a group of sensors/actuators via messages) usually leveraging general-purpose programming languages.

The initial steps with Node-RED seem promising. However, the design interface faces several challenges. The interest in using a visual programming interface lies behind the idea of simplifying how effects are connected. A tool like this is capable of connecting different platforms, managing the connection of several devices and give a higher-level control to the user. Every device (sensing or actuation) counts on its programming interface and communication protocol. As pointed out, tangible control options like those of the Soma Bits project, or even seen in other interaction instances such as music tabletop interfaces [Jordà et al., 2007], present interaction paradigms that are not mainly centred on clickable screens in a computer but wheels, physical buttons and interaction in 3d space. Knobs, sliders, controllers and elements that need to be physically moved, rotated or connected together lead tangible interactions. Node-RED, though, works nicely when prototyping effects, managing information processed by more intricate algorithms, and discerning how devices must communicate to each other or what the working ranges are for the parameters involved in their operation.

\subsection{Shape-changing breathing: supporting the design of a somaesthetic biosignal coupling}

In an effort to further extend the repertoire of body and self-awareness interaction modalities, a new design challenge of creating breathing-based effects has been undertaken recently by a joint team of interaction designers and biosignal researchers. In section 4.8, the work on creating couplings - i.e. artefacts, effects or mappings- that connect to the body in a relevant, intimate and evocative way is addressed by means of three examples. These are examples that counted on a group of designers / users / students, leading to rich biosignal interpretations that combined a collection of first-person experiences. Moreover, besides extending 
somaesthetic exercises and practices [Tsaknaki et al., 2019, Windlin et al., 2019] with a look into first-person physiology (section 4.7), couplings exploited the expertise of designers and design topics that surrounded the workshops conducted, i.e. synchrony and balance. In this section, ongoing work is introduced on the process of designing (via soma design) with breathing. This collaboration was initiated and led by researchers A. Jung, P. Karpashevich and K. Höök, counting on W. Primett contribution as well. For the first time, orchestration aspects such as interface/platform and the exploration of feature processing possibilities are brought to the discussion from the very start. The first-person soma approach remains a staple of this design work, in this case conducting several Feldenkrais exercises within the design sessions supported by body sheets (see, for instance, [Tsaknaki et al., 2019]). The study builds on recent progress that KTH has made, extending the previously developed interaction design toolkit. New "pixels" (modules) have been added to the Soma Bits [Windlin et al., 2019], consisting of little air pillows or pads that can be shaped conveniently, and which include pressure control.

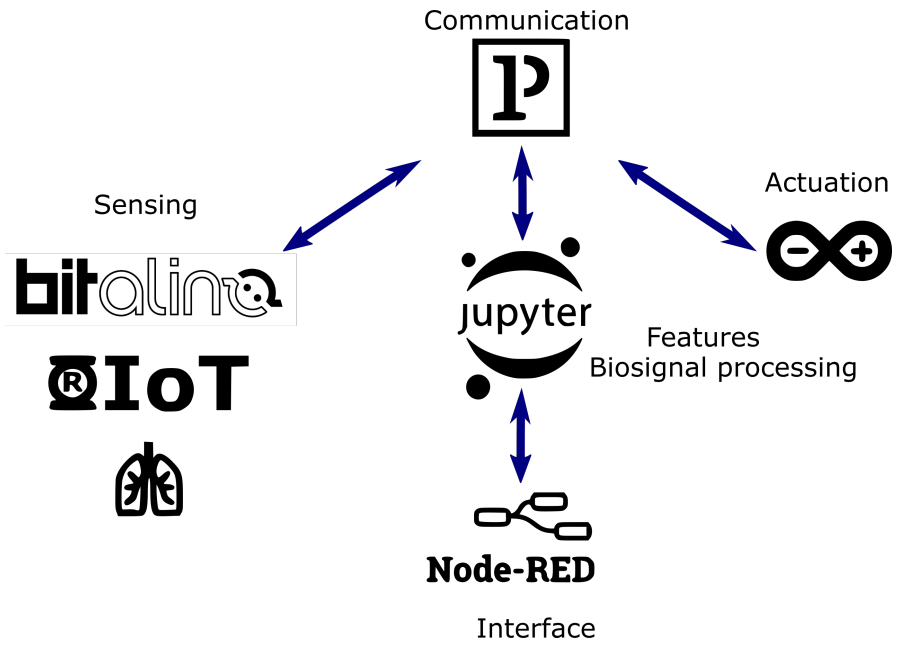

Figure 5.2: Shape-changing breathing communication workflow: Processing hub on top, managing OSC communication with (shown from left to right): sensors (R-loT), its data processing and configuration interface (jupyter and Node-RED) and the actuation effects (Arduino)

These enable the exploration of Arduino-based shape changing effects in the form of inflatables that are controlled through pressure valves, managed by Processing [Reas and Fry, 2003] instructions, and able to be felt by touch if attached to the body. In terms of input, a BITalino R-IoT [PLUX S.A. and IRCAM, 2017] 
with a respiratory inductive plethysmography sensor is used, leveraging the R-loT Wi-Fi communication capabilities and a sensing mechanism that is more robust than piezoelectric-based approaches to breathing activity monitoring. Real-time processing algorithms, the soma design structure of the sessions that let us designers - get attuned to the body and reflect on biodata, and the extended body actuation modules count now on orchestration interfaces.

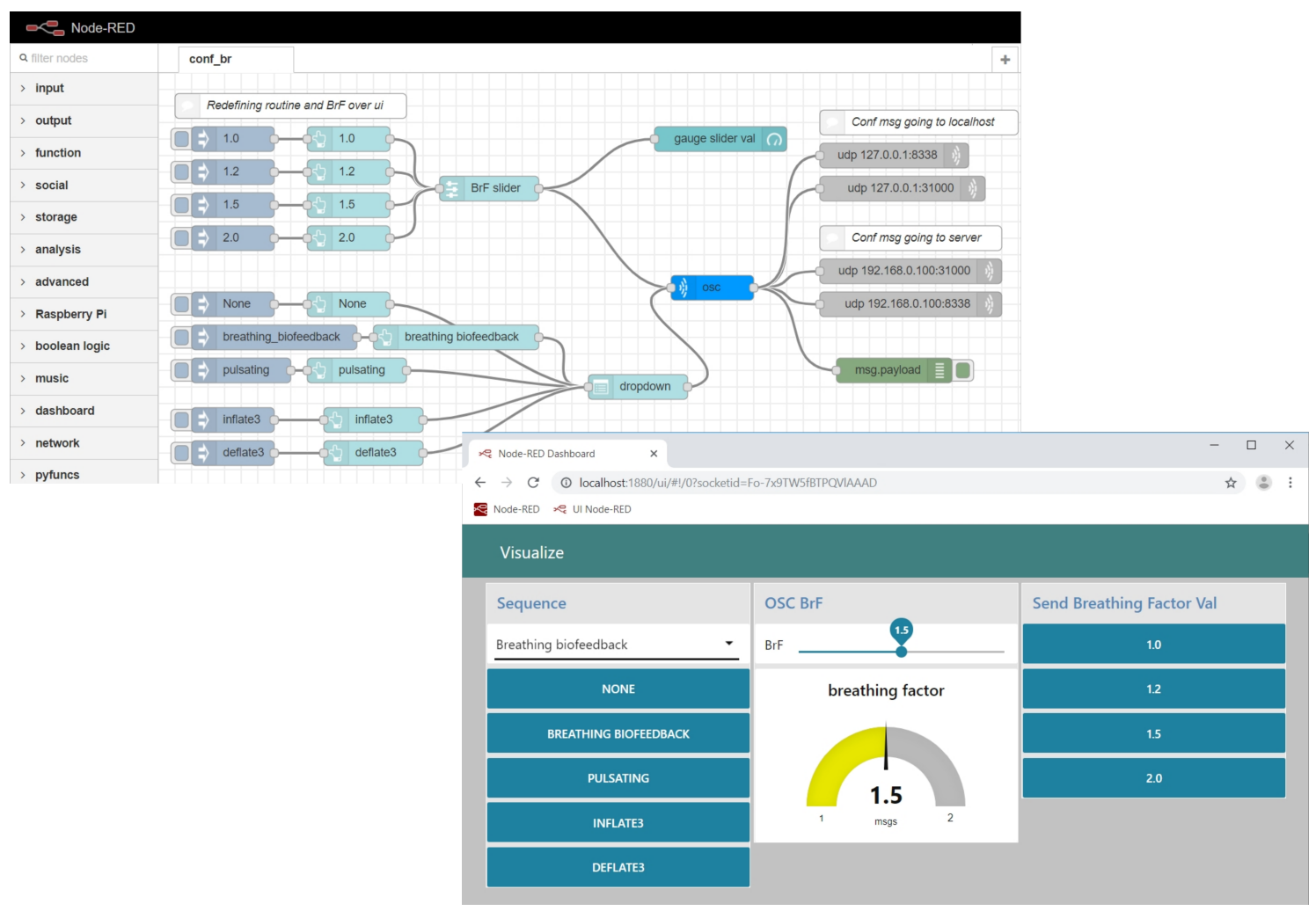

Figure 5.3: Node-RED UI configuration flow and browser configuration interface (bottom right) to be used during design sessions

In this experience, breathing is explored as an input modality, and pressurebased shape changes that can be felt are investigated, both with their rationale or inspiration behind, as explained below (section 5.3.1, section 5.3.2). In terms of tools supporting the design orchestration and exploration of mappings, the simple SomaBits structure takes a big leap now and incorporates extra steps to process data and communicate effects between input and output devices. Wi-Fi is chosen as the wireless approach to work with. Communication is managed by Processing scripts that act as a hub (see fig. 5.2). This hub sends and receives Open Sound 
Control (OSC) [Schmeder et al., 2010] messages to and from the sensing platform and the actuation platform. Actuation is managed and programmed via an Arduino that inherited the control mechanisms of the Soma Bits and extended functionalities with pressure monitoring that drive the inflation valves. The biosensing relies on the R-loT and is mainly accessed via Python instructions that run under Jupyter notebooks, communicating via OSC to the Processing hub when requested through a browser based Node-RED interface. In the Python code, the extracted signal processing features, such as current inhale/exhale state or breathing rate, are taken to build actuation instructions, e.g. applying inflation sequences that replicate or scale the measured cycles.

Table 5.1: Node-RED nodes used for the UI

\begin{tabular}{|c|c|l|}
\hline Node-RED node & Name & Function \\
\hline 1.0 & Input & Sends a numerical or text value input \\
\hline BrF slider & $\begin{array}{c}\text { Slider } \\
\text { controller }\end{array}$ & $\begin{array}{l}\text { Interface: Creates a slider with values to be } \\
\text { selected and sent to the BrF (breathing factor } \\
\text { configuration) }\end{array}$ \\
\hline dropdown & $\begin{array}{c}\text { Dropdown } \\
\text { selector }\end{array}$ & $\begin{array}{l}\text { Interface: Puts a dropdown with different modes to } \\
\text { be selected and sent }\end{array}$ \\
\hline osc & $\begin{array}{c}\text { OSC message } \\
\text { encoder }\end{array}$ & $\begin{array}{l}\text { Encodes messages in Open Sound Control format } \\
\text { before sending to a config script }\end{array}$ \\
\hline msg.payload & $\begin{array}{c}\text { Message } \\
\text { payload }\end{array}$ & Enables printing messages for debugging purposes \\
\hline udp 192.168.0.100:31000 & $\begin{array}{c}\text { UDP } \\
\text { server/client }\end{array}$ & Sends messages via a udp protocol \\
\hline gauge slider val & Gauge & $\begin{array}{l}\text { Interface: Monitor and display the current value of a } \\
\text { variable }\end{array}$ \\
\hline
\end{tabular}

Within the design sessions, a Node-RED (section 5.2.3) interface is used to change effects (type of output effect or intensity) at ease, showing that Node-RED not 
only allows to sequence or create biosignal-actuation mappings but rather, in this case, supports the change of settings during design sessions (see fig. 5.3, table 5.1). As a UI, Node-RED contributions add gauge nodes to count or plot current values on the browser, value inputs on click/trigger, dropdown menus and sliders, used alongside OSC encoding functionalities from other extension packages that facilitate communication to other platforms like the actuator that control the interaction or the sensor data processing software.

The Node-RED interfaces are part of a more ambitious, unconcluded, PLUX project Biofeatures Node-RED on integrating biosensing devices and the visual loT programming interface to enable interfacing directly with PLUX devices via the OpenSignals software and TCP communication, through BITalino R-IoT's automatically generated OSC messages, through BITalino APIs and Python servers running on the background (for example thanks to tools such as project pynored https://github.com/ghislainp/pynodered), or potentially exploring the possibility of creating native JavaScript Node-RED nodes working as if they were an extra application programming interface to the devices, with nodes for the data filtering or thresholding, as well as basic start/stop acquisition functionalities. The browser interfaces, much simpler, enable the launch of data capturing but, most importantly, the change of pre-coded actuation sequences or input breathing factor $(\mathrm{BrF})$ values to apply to the chosen 2-inflatable sequence (1. None/stop, 2.Breathing biofeedback scaling the measured breathing rate to a factor that dilates it, 3.Pulsate alternatively 4.Inflate/Deflate).

Currently, the breathing exploration is making it into the process of designing a garment that would integrate the pressure inflatables and the breathing (input sensing and interaction/self-awareness focus) [Jung et al., 2021]. An example of sharing instances that took place soma design sessions where breathing data was shared in the form of pressure inflatables can be seen (fig. 5.4).

\subsubsection{Deep Touch Pressure}

As with other biosignal-enhanced interaction design explorations, a design context was sought. Deep touch pressure (DTP) is a method used in sensory integration therapy which uses tools such as weighted garments and blankets, swaddling, or firm hugs to provide a firm pressure sensation to the body. Its calming effect seems to be due to stimulation of the parasympathetic nervous 

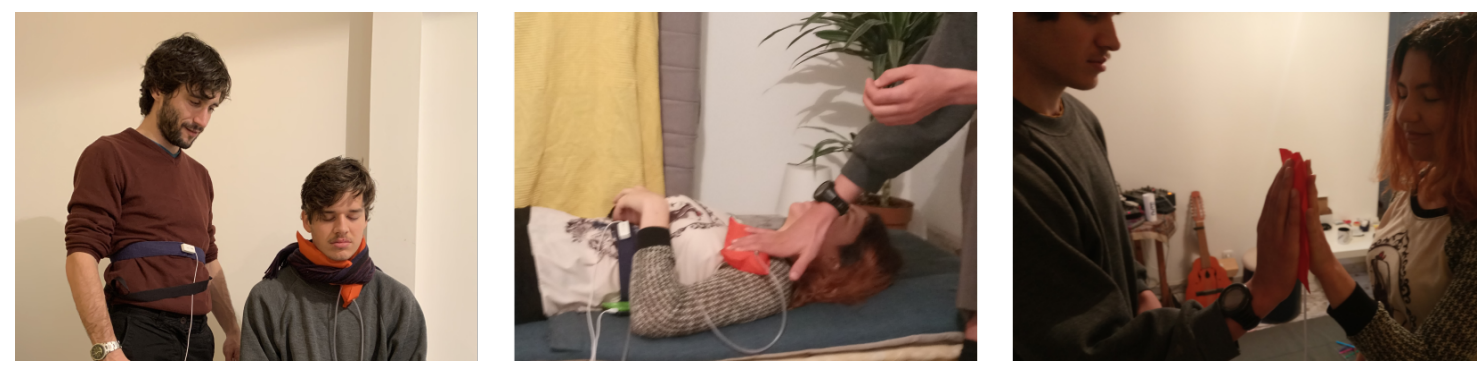

Figure 5.4: Instances of sharing a breathing biofeedback actuation via pressure inflatables on different body locations (neck, shoulder and hands)

system, which plays a significant role in anxiety management [Chen et al., 2013]. In therapy practice, DTP has been applied to increase attention [Fertel-Daly et al., 2001] and reduce anxiety symptoms [Krauss, 1987], particularly for children and students with autism spectrum disorders (ASD) [Lang et al., 2012].

Since DTP is considered a rather unconventional approach [Losinski et al., 2016], it has received limited attention in $\mathrm{HCl}$. Vaucelle et al [Vaucelle et al., 2009] developed a variety of prototypes for different forms of sensory stimulation, including a pressure vest containing pneumatic chambers. Such vests are the most common form of designs for deep touch pressure, and have also been applied to simulate hugs in long-distance interactions [Teh et al., 2008]. Another form of compression garments for DTP uses shape memory alloys which contract when heated, exerting pressure on the wearer's body [Duvall et al., 2019]. There have also been several commercial prototypes of inflatable vests [Tware, 2015, Squeasewear, 2014] which aim to help people with ASD and ADHD to manage anxiety, attention and sleep issues.

In gathering inspiration from what is known in therapy as deep touch pressure garments [Vaucelle et al., 2009, Tware, 2015], i.e. tangible grounding technology that leverages the use of pressure applied to the body, the issue of whether biosensing and somaesthetics could be utilised to revisit pressure-based actuation was posed. The resulting design exploration, uses a focus on the body to expose design limitations, feeding into the research of body self-awareness wearables and deep touch pressure interaction design. 


\subsubsection{Breathing as input}

Breathing activity can be captured as a physiological signal. As such, though, it entails a specific interaction constraint. Studies of physiology usability for $\mathrm{HCl}$ design [da Silva, 2017] try to shed some light on different affordances of the biosignals when deployed in real-world use case scenarios. As opposed to signals or physiological data with a longer tradition in the intersection of medical and $\mathrm{HCl}$ research such as heart rate, via electrocardiography (ECG) or bloodvolume pulse (BVP) sensing -which depict rhythms that are challenging to control-, respiration offers an interesting compromise of need and agency. Imagine, say, one represents physiological signals in a scale that rates their controllability, where highly involuntary sources such as brain synapses or digestion are considered to have the lowest rating. One needs to breathe to live. Breathing rhythms, however, can be altered at ease. On the other side of this interaction level, we would find movement tracking or muscle activity. Overly used in music performances or as voluntary input mechanisms to many interfaces, movement and electromyography (EMG) are physiology examples of signals that one can easily fine-tune, disrupt, control or even stop.

The controllability nature of breathing mixed with the relevance in calming techniques positioned it as an interesting interaction design focus candidate. Biosignals are not mere representations going up and down (see section 2.1.2), but a collection of shapes, durations and patterns to be assessed via elaborate features. In the exploration of breathing features, a breathing sequence was programmed early on, scaling inhale / exhale sequences to be felt through an inflatable. This turned out to be a mapping to stick to and refine.

In a paper by Tennent et. al [Tennent et al., 2011], sensory misalignment researchers explore the potential of breathing interfaces to control game inputs. Although biofeedback use in games is not new, they propose interfaces far from fitness-oriented use cases. Their approaches are based on breath flow measurement that draws upon modified gas masks. "Breathing is both autonomic and in part explicitly and quickly controllable." Although this cannot be detached from its physiological need and constraints. Part of their insight highlights that even when breathing appears as a highly controllable, high accuracy process, challenges such as sustained control for certain amounts of time emerge. The Tunnel Run interface enters into the design of an experience where a player tries to match 
the breathing data curve to that of an opponent. In an earlier work, Marshall et al. [Marshall et al., 2011] already identified the rich interplay between game-like elements, voluntary and involuntary control of breathing and skill build up against a machine in a breathing-based bucking bronco ride. As a side effect, subjects who engage in the game become more aware of their breathing.

Projects such as Un air de flûte [Lesobre, Vanessa, 2019] and its instrument interface aim to revolutionise respiratory failure rehabilitation, opening its design towards gamification and remote eHealth domains. In Just Breathe, a haptic interface is the basis of an stress intervention deployed in a driving scenario where participants are asked to regulate their states through breathing [Paredes et al., 2018].

\section{Processing breathing data}

Observing the raw data going up and down is informative, just as the conductor does in First-person Physiology experiences (section 4.7). This process invites the user to test the sensor limits and find out what it is that cannot be captured. In preparation of design sessions, an overview of features was sought, together with processing algorithms that could be created or tweaked as needed:

- Cycle duration (inhale/exhale)

- Breathing rate

- Instantaneous inhale/exhale state

- Rate of change (indicating how fast air is taken or released)

- Amplitude range (indicating how deep a breath is)

- Area (combining duration and depth of breath)

Having a breathing rate implementation turned out to be instrumental. The breathing interaction coupling explored presented here counted on the BioSPPY [Carreiras et al., 2015], enabling access to filtering and breathing rate estimation in an easy way. As soon as processed data became interaction ingredients to be fed to the actual inflatable, visual intuition was ready to be strengthened to proceed to try effects tangibly. This, however, called for a long trial and error process regarding the use of time. The resulting main actuation sequence is a 
biofeedback that computes the (averaged) duration of last inhale and exhales and requests it, scaled, on the inflatable side. 


\section{Chapter 6}

\section{Conclusion and final remarks}

In the final chapter of this thesis, it is worth to recap and revisit the initial challenge overview presented at the beginning of such research journey. The text found in the following section reflects back on the different strands of work merged within this investigation, adding insight in both the biosignal engineering perspective and the human-computer interaction design one. Pondering on the future research outlook (see section 5.1) and the open research paths only recently started to successfully address (such as orchestration, e.g. section 5.2) remarks on the thesis outcomes are given. To conclude, the contributions and research outcomes of this thesis are highlighted.

\subsection{General overview}

Research on mobile and wearable technologies that track behavioural, psychological, and contextual signals has recently gained momentum in the field of mental health. At the same time, the rise of personal sensing has garnered the interest of $\mathrm{HCl}$ research. In this thesis, the interest in affective technologies, body and emotional self-awareness experiences motivates the exploration of biosignals. At the beginning, the research focus revolved around finding ways in which physiology processing could be done efficiently and with the performance that competes with state-of-the-art techniques, considering its deployment within wireless devices or wearable health use cases. Echo state networks emerged as suitable candidates to be applied to physiology and wearable health, given their performance for specific tasks and the relatively undemanding computational 
needs that are attractive for devices that in principle do not count on high computational capabilities. While the ESN remarks apparently hold in the case of electrocardiography data analysis, it is yet to be seen which contexts or more traditional machine learning challenges are suitable for a method that is apparently good at exploiting the temporal dependencies of a cyclic signal (which is not the case of signals that are not as predictable or that are voluntarily controlled). ESNs face significant challenges that come to light when deployed on eHealth problems. As also exposed when addressing the processing of health records that either lack values or present variables not as densely sampled through time as an ECG, the limitations on temporal dependencies add up to the lack of clear guidelines on how to integrate data of different nature (metabolites, vitals, rates computed every hour), or required data fusion strategies. The case of sepsis classification exemplifies it (see section 3.4.1). These are limitations that other machine learning paradigms face too when trying to generalise to multivariate data. With respect to biosignal research advances that could impact the practice of $\mathrm{HCl}$ or interaction design more directly, the work towards real-time processing platforms and the study of complex features encompassing more than a single sensing modality seem more promising (as shown in the recent breathing interaction exploration experience, section 5.3).

With the goal of making biosignals available, two perspectives (developer and designer) are combined in this thesis to investigate ways in which biosignals can be exploited. Promoting interaction design with biosignals, research possibilities are sought, towards a body self-awareness focus and appropriating meaning-making processes moving beyond traditional ways to think of physiology. To achieve this, understanding current (and emerging) physiology processing platforms has been crucial. More importantly, with the adoption first-person soma design to integrate biosignals that are commonly used in ubiquitous low-cost personal sensing together with actuation mechanisms studied in $\mathrm{HCl}$, biodata has arguably been transformed into a material to design with. The biosignal-based design exploration, giving special attention to the sentient body and acknowledging alternative ways to address affect within interaction, culminated with a set of coupling examples, in which we demonstrate data mapping strategies between various devices in the context of bodily and emotion awareness. Through biosignal-actuation couplings, the role of orchestration defines the ways in which body input-output systems and meanings are put in place, the range of mappings and how they unfold. The two 
strands of work meet with interesting synergies in the study of biosignal-enhanced body interactions [Alfaras et al., 2020b]. The current efforts shown in section 5.3 are even more clear in highlighting how biosignals are available by means of biosignal processing, first-person soma design, biodata-based effects to be felt on the body and self-awareness goals more aligned with the psychotherapy practice. The design platforms achieved, in particular following the line started by the Soma Bits, shed light into directions that seem promising for the future of interaction design exploiting biodata. However, current prototypes still fall short in providing a smooth exploration of body-based effects. While the development of design toolkits is a work-in-progress, the many technologies involved imply a too large set of protocols and programming languages to render a simple design ecosystem. Research-wise, design paths accompanied by a soma design approach have proven to be very successful to approach a technology that is seldom seen as material. The future of affective technology interactions will be shaped by the development of design toolkits and platforms. Besides, throughout this thesis, the soma view has been complemented with that of sensory misalignment (section 4.6), inviting to the disruption of soma design itself with e.g. the inclusion of thrill and discomfort (section 4.5), and the consideration of how the body-centred design processes unfold back and forth between ends of bodily-inspired interaction design dimensions, i.e. moving along different levels of comfort/discomfort, intrusiveness (within/outside the body) or familiarity/strangeness that will spur further reflections on how to navigate this space.

On another front, the promising capabilities of design toolkits for self-reflection have also been studied by an example addressing affect representation as a core objective (section 4.4), using electrodermal activity sensing and body/material explorations but highlighting affect instead of overemphasising the chosen technology modalities to achieve its meaning. The combination of materials and technologies opens up arousal from a unique and personal perspective. While further research is taking the form of a coupling/orchestration augmentation (mainly motivated by the observations on the benefits of design support interfaces and interactive machine learning, section 5.2), the exploration of how affect information is appropriated through crafting practices is worth investigating further [Umair et al., 2020]. In this case, rather than extending a kit with more materials or refining it, and despite the possibilities of changing inputs and outputs to focus on different emotional information, there is room for addressing the very same personalised 
affect representations by studying prototyping behaviours and engagement in longer-term studies with other population samples. While project AffecTech [AffecTech, 2017] stresses the relevance of emotion regulation, with strong ties to cognitive behavioural psychotherapy, the soma design studies presented here pursued a more basic goal of turning biodata (not complex behaviours) malleable. However, as ThermoPixels expose with the emergence of emotion regulation in the users' hypothesised uses, and as the breathing exploration experience suggests (section 5.3), some contexts lie closer to the psychotherapy practice. Therefore, either the study of e.g. emotion regulation (backed up with multimodular psychophysiology monitoring to detect, characterise and expose its presence) or a less subtly addressed emotion regulation focus in a design process rather than affect representation offer possibilities worth studying further, with the potential to call for a more direct participation of therapy stakeholders as well.

In relation to ethics, AffecTech has also highlighted how $\mathrm{HCl}$ studies may benefit from a direct consideration of ethics during the design or research process [Sanches et al., 2019b]. This is somewhat echoed from the perspective of mental health applications available in mobile markets [Bowie et al., 2018, Bowie-DaBreo et al., 2020]. A broader discussion on the topic is presented in [Alfaras et al., 2020a]. This thesis, however, is no exception to a limited attention to ethics, handled with care when engaging participants, but only intermittently addressed as a main topic. Soma design is, to some extent, an approach to ethics, i.e. engaging with core values throughout the design process, also favoured via the first-person perspective that puts designers' - not solely final users' - feelings to the test during design, not after, and uses them, sharing and enriching an outcome that is broader or that represents the unalike multiple subjectivities brought forth in design session groups. The feelings of designers are not to be seen as less relevant but, quite the contrary, of importance if engaged in emotion work [Balaam et al., 2019] and when trying to instigate values in the designs or interactions created. These set the ground for future research endeavours including ethics, end-to-end.

\subsection{Thesis contributions}

The following text summarises the contributions and research outcomes of this thesis, divided into biosignal processing/engineering advances and humancomputer/interaction design ones, ultimately carrying a joint view exemplified with 
the final biosignal-actuation interaction artefacts or couplings.

\subsubsection{Engineering and biosignal development contributions}

In seeking ways to drive biosignal tracking research fronts, this thesis started by an eminently engineering focus that explored how biosignals are captured, processed, and represented, challenging how biosignals and its intrinsic physiological information are made available from a developer point of view. Contributions (and related objectives in brackets, see section 1.2) in this domain are the following:

\section{Computationally undemanding Echo state networks (ESNs) for the pro- cessing of biosignals and wearable health data [O3].}

This thesis drew on a background of studies carried out in the context of ESN architectures [Ortín et al., 2019, Alfaras et al., 2019a], i.e. simplistic recurrent neural networks, for the processing of biosignal data. This thesis further unpacked the use of ring-topology ESNs [Rodan and Tiňo, 2011] and created an ESNtools Python implementation [Alfaras et al., 2019b] which was applied to a worldwide data-driven sepsis [Reyna et al., 2020] classification challenge in which it competed with state of the art ML techniques. The proposed model in the context of the intensive care unit data, although beaten by ML paradigms that exploited mathematically robust path integrals [Morrill et al., 2019] or improved traditional ML techniques, is usable, easy to tweak and retrain. In turn, it is probably more aligned with (explainable) Al models that can be transparently assessed step by step, and helped underscore the insufficiently met goals of data fusion, data imputation for missing/faulty values and generalisation.

- Ring-topology Echo State Networks for ICU Sepsis Classification. [Alfaras et al., 2019c]

2. Real-time monitoring, processing and visualisation of biosignal data [O5, O4, O2].

Being based at a company that is devoted to the creation (and democratisation) of biosignal platforms, had a strong influence in the scope of this thesis, making it possible to link research knowledge with development efforts that tackle user's needs. This is to be seen, not only as an electronic device 
and sensing focus, but as a genuine interest of a company and research team in understanding how investigators, developers or even users who are not acquainted with biosignals can use them. In a collaboration led by LIBPhys at Universidade NOVA de Lisboa, it was demonstrated that heart rate variability processing (features, filtering) can be implemented in real-time biosignal visualisation platforms. The resulting proof-of-concept software add-on, delved into the temporal requirements that features of different nature posed for processing (e.g. temporal features and frequency features), and was successfully integrated in a beta plugin of the official PLUX OpenSignals software [PLUX S.A., 2018].

- Real-Time Approach to HRV Analysis. [Ramos et al., 2018]

\section{New metrics and descriptors for biosignal processing and pattern re- cognition $[\mathrm{O} 4, \mathrm{O} 5, \mathrm{O} 2]$.}

Continuing with the focus on enhancing biosignal processing capabilities, and in line with the goal of making biosignals available (to developers and researchers seeking to understand particular physiology aspects), the possibility of collaborating with a study centred on physical performance emerged as an interesting path to challenge the ways in which biosignals are utilised. A team led by LIBPhys - Universidade NOVA de Lisboa and collaborators from the Faculdade de Motricidade Humana of Universidade de Lisboa addressed the challenge of combining ECG and EMG data to capture the onset of fatigue in cycloergometry exercises. The resulting study successfully reviewed and characterised biosignal features and elaborated on those to propose individual fatigue descriptors. Moreover, a global fatigue descriptor was established, to characterise the level of fatigue with an associated probability, therefore setting a new metric for a complex phenomenon (fatigue). Completely aligned with the need to spur ways to make biosignals more available (in this case via elaborated new metrics capturing data patterns otherwise missed), this thesis specifically contributed to the assessment of the $\mathrm{ML}$ algorithm training procedures followed in the study.

- Fatigue evaluation through machine learning and a global fatigue descriptor. [Ramos et al., 2020] 
Beyond the scientific outcomes resulting from this strand of work, it is worth highlighting that the enrolment at a biosignal device manufacturer that was internally seeking ways to better engage biosignal users was a timely opportunity. This is reflected in the thesis' (and hosting company's) shift to researching tutorial materials that would accompany the biosignal acquisition users in their learning processes, to facilitate access to elaborate visualisations of the acquired biosignal data (see section 3.1 and [BITalino, 2018]), device status and inspire their own changes, tweaking ideas and data ownership. Such a transformative change, crystallised beyond this thesis as the company's commitment to strengthen the user learning process when dealing with biosignal acquisition (see the biosignalsnotebooks [PLUX S.A., 2020, Varandas et al., 2020] project). In this thesis, however, this approach is to be thanked for inspiring the Sensing Self ideas (see section 4.7) that permeated an $\mathrm{HCl}$ and self-awareness interaction design focus.

\subsection{2 $\mathrm{HCl}$ and Interaction design research contributions}

In seeking ways to drive biosignal research further, this thesis benefited from project AffecTech's [AffecTech, 2017, Repetto et al., 2017] focus on affective technologies and self-awareness, to investigate how making biosignals available for interaction designers could bring novelty in the ways people use biosignal acquisition devices or even spurring the development of biosignal interaction or feedback effects unthought of. The specific challenge of how biosignals can be made available to designers became a central point at the start of the thesis, inspiring design approaches and collaborations addressed in this text, often proposing developments that leveraged Do-it-yourself practices and design centred in the user's/researcher's own perspective. Since this interdisciplinary challenge entailed crossing fields and entering $\mathrm{HCl}$, the research of this thesis attempted to characterise relevant framings useful to support such a demanding journey. This is the case of the introduction to $\mathrm{HCl}$ waves (section 4.2.1), first-person and soma perspectives (section 4.2.2 and section 4.2.3) for interaction design, and a unique approach to affective technology and body/emotion self-awareness (section 4.3). Moreover, the first-person perspective is genuinely appropriated to inspire biosignal exploration (see First-person physiology, section 4.7). The thesis contributions (and related objectives in brackets, see section 1.2) in this domain are the following: 


\section{The ThermoPixels and the research on self-understanding and repres- entation of emotionally-charged information [07, O8].}

Thanks to a unique AffecTech [AffecTech, 2017] collaboration led by Lancaster University $\mathrm{HCl}$ researchers, a Do-it-yourself toolkit for the creation of EDA-based arousal representations was developed. Although the research teams who conceptualised the kit are exploring distribution and exploitation leads suitable for the toolkit, ThermoPixels was instrumental in this thesis for other reasons. In particular, the study around the kit contributed to realising that hybrid crafting combining assemble kits with unique user input are vehicles to unpack and strengthen affective self-awareness, and even a means to envision ways in which electrodermal activity information can be leveraged in daily emotional regulation contexts. ThermoPixels, building on very simple processing, and on appealing visuals exhibited by thermochromic materials, successfully paves future research in empowering the users with biosignal-arousal interpretation tools. Moreover, it is worth noting how ThermoPixels' workshops highlighted personalisation options and agency in deciding what to expose or not, without disregarding the difficulty entailed by the ambiguity of arousal (only referring to high/low emotional load and not its positive/negative valence).

- ThermoPixels:Toolkit for personalizing arousal-based interfaces through hybrid crafting. [Umair et al., 2020]

2. Dare to design. Extending interaction design from a self-centred perspective [06, 09].

Following on the fruitful interaction design line opened up by AffecTech and inspired by a theoretical background that was starting to be consolidated [Höök et al., 2018, Höök, 2018], collaborations with Lancaster $\mathrm{HCl}$ and KTH Stockholm Soma Design team helped focus the thesis on the task of leveraging first-person accounts surrounding technology and a holistic bodycentred perspective. As a result, significant discussion and insight is offered. This thesis contributed to assess, personally, existing issues surrounding discomfort in the use of personal tracking technologies exploiting a given biosignal (in this case electrodermal activity), such as concerns on being framed, being misunderstood or frustrated due to technology operation - either by frustration, malfunctioning or uses that are not in agreement 
with expectations while not being able to cope with the interaction/practice demands. This first (naive) approach, set the grounds for a more disruptive collaboration with Nottingham Mixed Reality Lab, not only taking the firstperson soma perspective to design with and challenging slow, gentle, soft qualities attached to it, but exploiting sensory misalignment (disturbing the habitual ways of addressing the user, taking confusing or even overwhelming tactics) to enhance a heightened sense of self that dares to open up design possibilities otherwise out of reach.

- Experiencing discomfort: Designing for affect from first-person perspective. [Umair et al., 2019a]

- Soma design and sensory misalignment. [Tennent et al., 2020]

\section{From Biodata to Somadata [O8, O9, O6, O1].}

This strand of work culminates the interdisciplinary research journey combining engineering and design investigation perspectives. Central to the thesis, the contributions in this line consist of a first-person account and approach at turning biosignal data available for interaction design teams, with a body-centred focus, using actuation effects and interaction ideas to understand and utilise biosignals and convey meanings in novel unprecedented ways. These took the form of evocative biosignal-actuation couplings that transformed EMG, EDA, ACC or Breathing signals into something malleable, overtly interpretable, tangible and able to be modified. Moreover, the thesis research surrounding these topics, helped underscore fundamental elements that must be present for a successful integration of biosignal inputs and evocative body-centred effects or outputs. In other words, with the discussion offered, it was possible to identify orchestration mechanisms and biosignal processing lines that could pave the way for future research avenues extending self-awareness technologies.

- From Biodata to Somadata [Alfaras et al., 2020b]

- Biosensing and actuation - Platforms coupling body input-output modalities for Affective Technologies. [Alfaras et al., 2020a]

- Exploring Awareness of Breathing through Deep Touch Pressure. [Jung et al., 2021] 


\section{Appendix: Research output list}

This PhD project has led to a collection of research outputs in the form of peer reviewed conference papers, journals and posters or other scientific dissemination actions wihtin the AffecTech project. The following list highlights the peer reviewed papers that make up the contents of this thesis:

(i) Ramos, G., Alfaras, M., and Gamboa, H. (2018). Real-Time Approach to HRV Analysis. In Proceedings of the 11th International Joint Conference on Biomedical Engineering Systems and Technologies BIOSTEC 2018. SCITEPRESS -Science and Technology Publications. http://doi.org/10.5220/ 0006641402080215

(ii) Ramos, G., Vaz, J. R., Mendonça, G. V., Pezarat-Correia,P., Rodrigues, J., Alfaras, M., and Gamboa, H.(2020). Fatigue evaluation through machine learning and a global fatigue descriptor. Journal of Healthcare Engineering, Hindawi 2020:1-18. https://doi .org/10.1155/2020/6484129

(iii) Alfaras, M., Varandas, R., and Gamboa, H. (2019). Ring-topology Echo State Networks for ICU Sepsis Classification. In 2019 Computing in Cardiology Conference (CinC). Computing in Cardiology. https://doi.org/10.22489/ CinC. 2019.327

(iv) Umair, M., Alfaras, M., Gamboa, H., and Sas, C. (2019).Experiencing discomfort: Designing for affect from first-person perspective.In Adjunct Proceedings of the 2019 ACM International Joint Conference on Pervasive and Ubiquitous Computing and Proceedings of the 2019 ACM International Symposium on Wearable Computers, UbiComp/ISWC '19 Adjunct,page 1093-1096, New York, NY, USA. Association for Computing Machinery. https://doi.org/10.1145/3341162.3354061 
(v) Tennent, P., Marshall, J., Tsaknaki, V., Windlin, C., Höök, K., and Alfaras, M. (2020). Soma design and sensory misalignment. In Proceedings of the $2020 \mathrm{CHI}$ Conference on Human Factors in Computing Systems, CHI '20, New York, NY, USA. Association for Computing Machinery. https: //doi.org/10.1145/3313831.3376812

(vi) Alfaras, M., Tsaknaki, V., Sanches, P., Windlin, C., Umair, M.,Sas, C., and Höök, K. (2020). From Biodata to Somadata. In Proceedings of the 2020 CHI Conference on Human Factors in Computing Systems, CHI '20, New York, NY, USA. Association for Computing Machinery. http://dx. doi .org/ $10.1145 / 3313831.3376684$

(vii) Umair, M., Sas, C., and Alfaras, M. (2020). ThermoPixels:Toolkit for personalizing arousal-based interfaces through hybrid crafting. In Proceedings of the 2020 ACM Designing Interactive Systems Conference, DIS'20, page 1017-1032, New York, NY, USA. Association for Computing Machinery. https://doi.org/10.1145/3357236.3395512

(viii) Alfaras, M., Primett, W., Umair, M., Windlin, C., Karpashevich, P., Chalabianloo, N., Bowie, D., Sas, C., Sanches, P., Höök, K., Ersoy, C., and Gamboa, $\mathrm{H}$. (2020). Biosensing and actuation - Platforms coupling body inputoutput modalities for Affective Technologies.Sensors, 20(21):5968. MDPI. https://doi.org/10.3390/s20215968

(ix) Jung, A., Alfaras, M., Karpashevich, P., Primett, W., Höök, K. (2021). Exploring Awareness of Breathing through Deep Touch Pressure. In Proceedings of the $2021 \mathrm{CHI}$ Conference on Human Factors in Computing Systems, CHI '21, New York, NY, USA. Association for Computing Machinery. https://doi.org/10.1145/3411764.3445533

\section{Follow-up works}

The work on soma design and sensory misalignment evolved throughout the course of this thesis, leading to more elaborate contributions not covered in this text.

- Tennent, P., Höök, K., Benford, S., Tsaknaki, V., Ståhl, A., Dauden Roquet, C., Windlin, C., Sanches, P., Marshall, J., Li, C., Martinez Avila, J., Alfaras, 
M., Umair, M. and Zhou, F. (2021). Articulating Soma Experiences Using Trajectories. In Proceedings of the $2021 \mathrm{CHI}$ Conference on Human Factors in Computing Systems, CHI '21, New York, NY, USA. Association for Computing Machinery. https://doi.org/10.1145/3411764.3445482,

- Höök, K., Benford, S., Tennent, P., Tsaknaki, V., Alfaras, M., Martinez Avila, J., Li, C., Marshall, J., Daudén Roquet, C., Sanches, P., Ståhl, A., Umair, M., Windlin, C. and Zhou, F. (2021). Unpacking non-dualistic design: the soma design case. (accepted) ACM Transactions on Computer-Human Interaction - TOCHI.

\section{Concurrent works}

Additionally, the following works, appeared during the PhD project, motivated part of the research questions addressed around Machine Learning and computationally undemanding paradigms suitable for health wearables to process physiology.

- Ortín, S., Soriano, M. C., Alfaras, M., and Mirasso, C. R. (2019). Automated real-time method for ventricular heartbeat classification. Computer Methods and Programs in Biomedicine, 169:1-8 https://doi .org/10.1016/j .cmpb . 2018.11.005

- Alfaras, M., Soriano, M. C., and Ortín, S. (2019). A fast machine learning model for ECG-based heartbeat classification and arrhythmia detection. Frontiers in Physics, 7. https://doi.org/10.3389/fphy.2019.00103

\section{Additional publications}

Throughout the AffecTech project, other works and research efforts intersecting with Biosignals, Psychophysiology/Psychotherapy, Robotics and emotions were inspired, although not directly addressed in this thesis.

- Cartosio, L., Robers, M., Alfaras, M., Pobil, A. What Are Emotions? And Do Robots Need Them? An Interdisciplinary Review (CyPsy'25 poster / paper in preparation) 
- Alfaras, M. Emotion technology: Falling Short Presented at the 2020 ACM Designing Interactive Systems Conference, DIS'20 in the workshop "Mental Wellbeing: Future Agenda Drawing from Design, HCl and Big Data" https ://doi .org/10.1145/3393914.3395920 


\section{Bibliography}

[AAMI / ANSI, 2012] AAMI / ANSI (2012). Ec57: 2012 testing and reporting performance results of cardiac rhythm and st segment measurement algorithms. American National Standards Institute, Arlington, VA, USA.

[Abdullah et al., 2016] Abdullah, S., Matthews, M., Frank, E., Doherty, G., Gay, G., and Choudhury, T. (2016). Automatic detection of social rhythms in bipolar disorder. Journal of the American Medical Informatics Association, 23(3):538543.

[Acharya et al., 2006] Acharya, U. R., Joseph, K. P., Kannathal, N., Lim, C. M., and Suri, J. S. (2006). Heart rate variability: a review. Medical \& Biological Engineering \& Computing, 44(12):1031-1051.

[AffecTech, 2017] AffecTech (2017). AffecTech: Personal technologies for affective health. https://www . affectech.org/. Accessed: 2019-06-30.

[AHA / ECRI, 2003] AHA / ECRI (2003). Ventricular arrhythmia ECG database.

[Akner-Koler and Ranjbar, 2016] Akner-Koler, C. and Ranjbar, P. (2016). Integrating sensitizing labs in an educational design process for haptic interaction. FormAkademisk - forskningstidsskrift for design og designdidaktikk, 9(2).

[Alfaras et al., 2020a] Alfaras, M., Primett, W., Umair, M., Windlin, C., Karpashevich, P., Chalabianloo, N., Bowie, D., Sas, C., Sanches, P., Höök, K., Ersoy, C., and Gamboa, H. (2020a). Biosensing and actuation - platforms coupling body input-output modalities for affective technologies. Sensors, 20(21).

[Alfaras et al., 2019a] Alfaras, M., Soriano, M. C., and Ortín, S. (2019a). A fast machine learning model for ECG-based heartbeat classification and arrhythmia detection. Frontiers in Physics, 7. 
[Alfaras et al., 2020b] Alfaras, M., Tsaknaki, V., Sanches, P., Windlin, C., Umair, M., Sas, C., and Höök, K. (2020b). From biodata to somadata. In Proceedings of the $2020 \mathrm{CHI}$ Conference on Human Factors in Computing Systems, CHI '20, page 1-14, New York, NY, USA. Association for Computing Machinery.

[Alfaras et al., 2019b] Alfaras, M., Varandas, R., and Gamboa, H. (2019b). pnet ESN tools. https://github.com/malfarasplux/pnet2019/tree/master/ ESNtools.

[Alfaras et al., 2019c] Alfaras, M., Varandas, R., and Gamboa, H. (2019c). Ringtopology echo state networks for ICU sepsis classification. In 2019 Computing in Cardiology Conference (CinC). Computing in Cardiology.

[Appeltant et al., 2011] Appeltant, L., Soriano, M., der Sande, G. V., Danckaert, J., Massar, S., Dambre, J., Schrauwen, B., Mirasso, C., and Fischer, I. (2011). Information processing using a single dynamical node as complex system. Nature Communications, 2(1).

[Ayzenberg et al., 2012] Ayzenberg, Y., Hernandez Rivera, J., and Picard, R. (2012). Feel: Frequent eda and event logging - a mobile social interaction stress monitoring system. In CHI '12 Extended Abstracts on Human Factors in Computing Systems, CHI EA '12, page 2357-2362, New York, NY, USA. Association for Computing Machinery.

[Balaam et al., 2019] Balaam, M., Comber, R., Clarke, R. E., Windlin, C., Ståhl, A., Höök, K., and Fitzpatrick, G. (2019). Emotion work in experience-centered design. In Proceedings of the $2019 \mathrm{CHI}$ Conference on Human Factors in Computing Systems, CHI '19, page 1-12, New York, NY, USA. Association for Computing Machinery.

[Bardram and Matic, 2020] Bardram, J. E. and Matic, A. (2020). A decade of ubiquitous computing research in mental health. IEEE Pervasive Computing, 19(1):62-72.

[Batista et al., 2019] Batista, D., da Silva, H. P., Fred, A., Moreira, C., Reis, M., and Ferreira, H. A. (2019). Benchmarking of the BITalino biomedical toolkit against an established gold standard. Healthcare Technology Letters, 6(2):32-36. 
[Beauchaine and Thayer, 2015] Beauchaine, T. P. and Thayer, J. F. (2015). Heart rate variability as a transdiagnostic biomarker of psychopathology. International Journal of Psychophysiology, 98(2):338-350.

[Bell et al., 2005] Bell, G., Blythe, M., and Sengers, P. (2005). Making by making strange: Defamiliarization and the design of domestic technologies. ACM Trans. Comput.-Hum. Interact., 12(2):149-173.

[BITalino, 2013] BITalino (2013). Bitalino. http://www. bitalino.com.

[BITalino, 2017] BITalino (2017). ServerBIT (r)evolution. https://github.com/ BITalinoWorld/revolution-python-serverbit.

[BITalino, 2018] BITalino (2018). BITalino Hands-on. https://github.com/ BITalinoWorld/python-lab-guides/tree/master/BITalino\%20Hands-on.

[Blevis et al., 2014] Blevis, E., Chow, K., Koskinen, I., Poggenpohl, S., and Tsin, C. (2014). Billions of interaction designers. Interactions, 21(6):34-41.

[Bødker, 2015] Bødker, S. (2015). Third-wave HCl, 10 years later-participation and sharing. Interactions, 22(5):24-31.

[Boehner et al., 2007] Boehner, K., DePaula, R., Dourish, P., and Sengers, P. (2007). How emotion is made and measured. International Journal of HumanComputer Studies, 65(4):275-291.

[Bowie et al., 2018] Bowie, D., Sunram-Lea, S.-I., and Iles-Smith, H. (2018). A systemic ethical framework for mobile mental health: From design to implementation. nihr mindtech mic national symposium 2018: Improving lives with digital mental healthcare. https://eprints.lancs.ac.uk/id/eprint/131847/1/A_ systemic_ethical_framework_for_mobile_mental_health_From_design_to_ implementation.pdf.

[Bowie-DaBreo et al., 2020] Bowie-DaBreo, D., Sünram-Lea, S. I., Sas, C., and lles-Smith, H. (2020). Evaluation of treatment descriptions and alignment with clinical guidance of apps for depression on app stores: Systematic search and content analysis. JMIR Formative Research (submitted). 
[Bradley and Lang, 2017] Bradley, M. M. and Lang, P. J. (2017). International affective picture system. In Encyclopedia of Personality and Individual Differences, pages 1-4. Springer International Publishing.

[Brunner et al., 2013] Brunner, D., Soriano, M. C., Mirasso, C. R., and Fischer, I. (2013). Parallel photonic information processing at gigabyte per second data rates using transient states. Nature Communications, 4(1).

[Byrne et al., 2018] Byrne, R., Marshall, J., and Mueller, F. F. (2018). Ar fighter: Using hmds to create vertigo play experiences. In Proceedings of the 2018 Annual Symposium on Computer-Human Interaction in Play, CHI PLAY '18, page 45-57, New York, NY, USA. Association for Computing Machinery.

[Cabibihan et al., 2016] Cabibihan, J.-J., Javed, H., Aldosari, M., Frazier, T., and Elbashir, H. (2016). Sensing technologies for autism spectrum disorder screening and intervention. Sensors, 17(12):46.

[Can et al., 2019] Can, Y. S., Chalabianloo, N., Ekiz, D., and Ersoy, C. (2019). Continuous stress detection using wearable sensors in real life: Algorithmic programming contest case study. Sensors, 19(8):1849.

[Can et al., 2020a] Can, Y. S., Chalabianloo, N., Ekiz, D., Fernandez-Alvarez, J., Repetto, C., Riva, G., Iles-Smith, H., and Ersoy, C. (2020a). Real-life stress level monitoring using smart bands in the light of contextual information. IEEE Sensors Journal, pages 1-1.

[Can et al., 2020b] Can, Y. S., Chalabianloo, N., Ekiz, D., Fernandez-Alvarez, J., Riva, G., and Ersoy, C. (2020b). Personal stress-level clustering and decisionlevel smoothing to enhance the performance of ambulatory stress detection with smartwatches. IEEE Access, 8:38146-38163.

[Carlo Ratti Associati, 2016] Carlo Ratti Associati (2016). Project lift-bit. https: //carloratti.com/project/lift-bit/.

[Carney et al., 2020] Carney, M., Webster, B., Alvarado, I., Phillips, K., Howell, N., Griffith, J., Jongejan, J., Pitaru, A., and Chen, A. (2020). Teachable machine: Approachable web-based tool for exploring machine learning classification. In 
Extended Abstracts of the $2020 \mathrm{CHI}$ Conference on Human Factors in Computing Systems, CHI EA '20, page 1-8, New York, NY, USA. Association for Computing Machinery.

[Carreiras et al., 2015] Carreiras, C., Alves, A. P., Lourenço, A., Canento, F., Silva, H., Fred, A., et al. (2015). BioSPPy: Biosignal processing in Python. [Online; accessed jtoday $i]$.

[Castaneda et al., 2018] Castaneda, D., Esparza, A., Ghamari, M., Soltanpur, C., and Nazeran, H. (2018). A review on wearable photoplethysmography sensors and their potential future applications in health care. International journal of biosensors \& bioelectronics, 4(4):195.

[Chang et al., 2019] Chang, Y., Rubin, J., Boverman, G., Vij, S., Rahman, A., Natarajan, A., and Parvaneh, S. (2019). A multi-task imputation and classification neural architecture for early prediction of sepsis from multivariate clinical time series. In 2019 Computing in Cardiology Conference (CinC). Computing in Cardiology.

[Chen et al., 2013] Chen, H.-Y., Yang, H., Chi, H.-J., and Chen, H.-M. (2013). Physiological effects of deep touch pressure on anxiety alleviation: The weighted blanket approach. Journal of Medical and Biological Engineering, 33:463-470.

[ChuDuc et al., 2013] ChuDuc, H., NguyenPhan, K., and NguyenViet, D. (2013). A review of heart rate variability and its applications. APCBEE Procedia, 7:80-85.

[Clawson et al., 2015] Clawson, J., Pater, J. A., Miller, A. D., Mynatt, E. D., and Mamykina, L. (2015). No longer wearing: Investigating the abandonment of personal health-tracking technologies on craigslist. In Proceedings of the 2015 ACM International Joint Conference on Pervasive and Ubiquitous Computing, UbiComp '15, page 647-658, New York, NY, USA. Association for Computing Machinery.

[Cross, 1992] Cross, N. (1992). Design ability. NA - Nordic Journal of Architectural Research, 5(4).

[da Silva, 2017] da Silva, H. P. (2017). The biosignal C.A.O.S.: Reflections on the usability of physiological sensing for human-computer interaction practitioners and researchers. In Ibáñez, J., González-Vargas, J., Azorín, J. M., Akay, M., 
and Pons, J. L., editors, Converging Clinical and Engineering Research on Neurorehabilitation II, pages 807-811, Cham. Springer International Publishing.

[da Silva, 2018] da Silva, H. P. (2018). Physiological sensing now open to the world: New resources are allowing us to learn, experiment, and create imaginative solutions for biomedical applications. IEEE Pulse, 9(2):9-11.

[da Silva et al., 2014] da Silva, H. P., Fred, A., and Martins, R. (2014). Biosignals for everyone. IEEE Pervasive Computing, 13(4):64-71.

[Day et al., 2002] Day, B. L., Guerraz, M., and Cole, J. (2002). Sensory interactions for human balance control revealed by galvanic vestibular stimulation. In Advances in Experimental Medicine and Biology, pages 129-137. Springer US.

[Devendorf et al., 2016] Devendorf, L., Lo, J., Howell, N., Lee, J. L., Gong, N.-W., Karagozler, M. E., Fukuhara, S., Poupyrev, I., Paulos, E., and Ryokai, K. (2016). "i don't want to wear a screen": Probing perceptions of and possibilities for dynamic displays on clothing. In Proceedings of the $2016 \mathrm{CHI}$ Conference on Human Factors in Computing Systems, CHI '16, page 6028-6039, New York, NY, USA. Association for Computing Machinery.

[Dourish, 2001] Dourish, P. (2001). Where the Action Is: The Foundations of Embodied Interaction, page 256. MIT Press, Cambridge, MA, USA.

[Du et al., 2017] Du, C., Cai, F., Zidan, M. A., Ma, W., Lee, S. H., and Lu, W. D. (2017). Reservoir computing using dynamic memristors for temporal information processing. Nature Communications, 8(1).

[Du et al., 2019] Du, J. A., Sadr, N., and de Chazal, P. (2019). Automated prediction of sepsis onset using gradient boosted decision trees. In 2019 Computing in Cardiology Conference (CinC). Computing in Cardiology.

[Duarte and Baranauskas, 2016] Duarte, E. F. and Baranauskas, M. C. C. (2016). Revisiting the three $\mathrm{HCl}$ waves: A preliminary discussion on philosophy of science and research paradigms. In Proceedings of the 15th Brazilian Symposium on Human Factors in Computing Systems, IHC '16, New York, NY, USA. Association for Computing Machinery.

[Dunn et al., 2018] Dunn, J., Runge, R., and Snyder, M. (2018). Wearables and the medical revolution. Personalized Medicine, 15(5):429-448. 
[Duvall et al., 2019] Duvall, J. C., Schleif, N., Dunne, L. E., and Holschuh, B. (2019). Dynamic Compression Garments for Sensory Processing Disorder Treatment Using Integrated Active Materials. Journal of Medical Devices, 13(2). 021001.

[Ehrsson, 2005] Ehrsson, H. H. (2005). Touching a rubber hand: Feeling of body ownership is associated with activity in multisensory brain areas. Journal of Neuroscience, 25(45):10564-10573.

[Elblaus et al., 2015] Elblaus, L., Tsaknaki, V., Lewandowski, V., and Bresin, R. (2015). Nebula: An interactive garment designed for functional aesthetics. In Proceedings of the 33rd Annual ACM Conference Extended Abstracts on Human Factors in Computing Systems, CHI EA '15, page 275-278, New York, NY, USA. Association for Computing Machinery.

[eMEN, 2019] eMEN (2019). eMEN: e-mental health innovation and transnational implementation platform North West Europe. https://ec.europa.eu/regional_policy/en/projects/France/ emen-using-technology-to-improve-europes-mental-health.

Accessed: 2019-05-10.

[EMPATICA, 2020] EMPATICA (2020). E4 wristband. https://e4. empatica.com/ e4-wristband.

[Fails and Olsen, 2003] Fails, J. A. and Olsen, D. R. (2003). Interactive machine learning. In Proceedings of the 8th International Conference on Intelligent User Interfaces, IUI '03, page 39-45, New York, NY, USA. Association for Computing Machinery.

[Faude et al., 2009] Faude, O., Kindermann, W., and Meyer, T. (2009). Lactate threshold concepts. Sports Medicine, 39(6):469-490.

[Fdili Alaoui, 2019] Fdili Alaoui, S. (2019). Making an interactive dance piece: Tensions in integrating technology in art. In Proceedings of the 2019 on Designing Interactive Systems Conference, DIS '19, pages 1195-1208, New York, NY, USA. ACM.

[Fernaeus and Sundström, 2012] Fernaeus, Y. and Sundström, P. (2012). The material move how materials matter in interaction design research. In Proceed- 
ings of the Designing Interactive Systems Conference, DIS '12, page 486-495, New York, NY, USA. Association for Computing Machinery.

[Fertel-Daly et al., 2001] Fertel-Daly, D., Bedell, G., and Hinojosa, J. (2001). Effects of a Weighted Vest on Attention to Task and Self-Stimulatory Behaviors in Preschoolers With Pervasive Developmental Disorders. American Journal of Occupational Therapy, 55(6):629-640.

[Fiebrink, 2011] Fiebrink, R. A. (2011). Real-Time Human Interaction with Supervised Learning Algorithms for Music Composition and Performance. PhD thesis, Computer Science Dept. Engineering Quadrangle Princeton, USA.

[Garcia-Palacios et al., 2013] Garcia-Palacios, A., Herrero, R., Belmonte, M., Castilla, D., Guixeres, J., Molinari, G., Baños, R., and Botella, C. (2013). Ecological momentary assessment for chronic pain in fibromyalgia using a smartphone: A randomized crossover study. European Journal of Pain, 18(6):862872.

[Giaccardi and Karana, 2015] Giaccardi, E. and Karana, E. (2015). Foundations of materials experience: An approach for $\mathrm{HCl}$. In Proceedings of the 33rd Annual ACM Conference on Human Factors in Computing Systems, CHI '15, page 2447-2456, New York, NY, USA. Association for Computing Machinery.

[Giannakakis et al., 2019] Giannakakis, G., Grigoriadis, D., Giannakaki, K., Simantiraki, O., Roniotis, A., and Tsiknakis, M. (2019). Review on psychological stress detection using biosignals. IEEE Transactions on Affective Computing, pages $1-1$.

[Goldberger et al., 2000] Goldberger, A. L., Amaral, L. A. N., Glass, L., Hausdorff, J. M., Ivanov, P. C., Mark, R. G., Mietus, J. E., Moody, G. B., Peng, C.-K., and Stanley, H. E. (2000). PhysioBank, PhysioToolkit, and PhysioNet: Components of a new research resource for complex physiologic signals. Circulation, 101(23):215-220.

[Goldich, 2014] Goldich, G. (2014). 12-lead ECGs, part i. Nursing, 44(8):28-34.

[Golsteijn et al., 2013] Golsteijn, C., van den Hoven, E., Frohlich, D., and Sellen, A. (2013). Hybrid crafting: towards an integrated practice of crafting with physical and digital components. Personal and Ubiquitous Computing, 18(3):593-611. 
[Gross, 1998] Gross, J. J. (1998). The emerging field of emotion regulation: An integrative review. Review of General Psychology, 2(3):271-299.

[Grove - Seeed Studio, 2014] Grove - Seeed Studio (2014). Grove GSR sensor. https://wiki.seeedstudio.com/Grove-GSR_Sensor/.

[Hao et al., 2014] Hao, Y., Budd, J., Jackson, M. M., Sati, M., and Soni, S. (2014). A visual feedback design based on a brain-computer interface to assist users regulate their emotional state. In CHI '14 Extended Abstracts on Human Factors in Computing Systems, CHI EA '14, page 2491-2496, New York, NY, USA. Association for Computing Machinery.

[Harrison et al., 2007] Harrison, S., Tatar, D., and Sengers, P. (2007). The three paradigms of HCl. In Alt.Chi. SIGCHI Conference on Human Factors in Computing Systems, New York, NY, USA. Association for Computing Machinery.

[Heikenfeld et al., 2018] Heikenfeld, J., Jajack, A., Rogers, J., Gutruf, P., Tian, L., Pan, T., Li, R., Khine, M., Kim, J., Wang, J., and Kim, J. (2018). Wearable sensors: modalities, challenges, and prospects. Lab Chip, 18:217-248.

[Hollis et al., 2017] Hollis, V., Konrad, A., Springer, A., Antoun, M., Antoun, C., Martin, R., and Whittaker, S. (2017). What does all this data mean for my future mood? actionable analytics and targeted reflection for emotional well-being. Hum.-Comput. Interact., 32(5-6):208-267.

[Höök, 2012] Höök, K. (2012). Affective computing: Affective computing, affective interaction and technology as experience. In The Encyclopedia of HumanComputer Interaction, 2nd Ed., chapter 12. The Interaction- Design.org Foundation, Aarhus, Denmark.

[Höök, 2018] Höök, K. (2018). Designing with the Body: Somaesthetic Interaction Design (Design Thinking, Design Theory). The MIT Press, Cambridge, MA.

[Höök, 2020] Höök, K. (2020). Soma design - intertwining aesthetics, ethics and movement. In Proceedings of the Fourteenth International Conference on Tangible, Embedded, and Embodied Interaction, TEl '20, page 1, New York, NY, USA. Association for Computing Machinery. 
[Höök et al., 2018] Höök, K., Caramiaux, B., Erkut, C., Forlizzi, J., Hajinejad, N., Haller, M., Hummels, C., Isbister, K., Jonsson, M., Khut, G., Loke, L., Lottridge, D., Marti, P., Melcer, E., Müller, F., Petersen, M., Schiphorst, T., Segura, E., Ståhl, A., Svanæs, D., Tholander, J., and Tobiasson, H. (2018). Embracing first-person perspectives in soma-based design. Informatics, 5(1):8.

[Höök et al., 2019] Höök, K., Eriksson, S., Louise Juul Søndergaard, M., Ciolfi Felice, M., Campo Woytuk, N., Kilic Afsar, O., Tsaknaki, V., and Ståhl, A. (2019). Soma design and politics of the body. In Proceedings of the Halfway to the Future Symposium 2019, HTTF 2019, New York, NY, USA. Association for Computing Machinery.

[Houben et al., 2016] Houben, S., Golsteijn, C., Gallacher, S., Johnson, R., Bakker, S., Marquardt, N., Capra, L., and Rogers, Y. (2016). Physikit: Data engagement through physical ambient visualizations in the home. In Proceedings of the $2016 \mathrm{CHI}$ Conference on Human Factors in Computing Systems, CHI '16, page 1608-1619, New York, NY, USA. Association for Computing Machinery.

[Howell et al., 2018a] Howell, N., Chuang, J., De Kosnik, A., Niemeyer, G., and Ryokai, K. (2018a). Emotional biosensing: Exploring critical alternatives. Proc. ACM Hum.-Comput. Interact., 2(CSCW).

[Howell et al., 2016] Howell, N., Devendorf, L., Tian, R. K., Vega Galvez, T., Gong, N.-W., Poupyrev, I., Paulos, E., and Ryokai, K. (2016). Biosignals as social cues: Ambiguity and emotional interpretation in social displays of skin conductance. In Proceedings of the 2016 ACM Conference on Designing Interactive Systems, DIS '16, page 865-870, New York, NY, USA. Association for Computing Machinery.

[Howell et al., 2018b] Howell, N., Devendorf, L., Vega Gálvez, T. A., Tian, R., and Ryokai, K. (2018b). Tensions of data-driven reflection: A case study of real-time emotional biosensing. In Proceedings of the $2018 \mathrm{CHI}$ Conference on Human Factors in Computing Systems, page 1-13, New York, NY, USA. Association for Computing Machinery.

[Howell et al., 2019] Howell, N., Niemeyer, G., and Ryokai, K. (2019). Lifeaffirming biosensing in public: Sounding heartbeats on a red bench. In Proceed- 
ings of the 2019 CHI Conference on Human Factors in Computing Systems, CHI '19, page 1-16, New York, NY, USA. Association for Computing Machinery.

[Hurst and Tobias, 2011] Hurst, A. and Tobias, J. (2011). Empowering individuals with do-it-yourself assistive technology. In The Proceedings of the 13th International ACM SIGACCESS Conference on Computers and Accessibility, ASSETS '11, page 11-18, New York, NY, USA. Association for Computing Machinery.

[Jaeger, 2001] Jaeger, H. (2001). The "echo state" approach to analysing and training recurrent neural networks-with an erratum note. GMD Technical Report, 148(34):13.

[Jaeger, 2007] Jaeger, H. (2007). Echo state network. Scholarpedia, 2(9):2330.

[Jones et al., 2016] Jones, T., Moore, T., and Choo, J. (2016). The impact of virtual reality on chronic pain. PLOS ONE, 11(12):e0167523.

[Jordà et al., 2007] Jordà, S., Geiger, G., Alonso, M., and Kaltenbrunner, M. (2007). The reactable: Exploring the synergy between live music performance and tabletop tangible interfaces. In Proceedings of the 1st International Conference on Tangible and Embedded Interaction, TEI '07, page 139-146, New York, NY, USA. Association for Computing Machinery.

[Jung et al., 2021] Jung, A., Alfaras, M., Karpashevich, P., Primett, W., and Höök, K. (2021). Exploring awareness of breathing through deep touch pressure. In Proceedings of the $2021 \mathrm{CHI}$ Conference on Human Factors in Computing Systems, CHI '21, New York, NY, USA. Association for Computing Machinery.

[Jung and Stolterman, 2012] Jung, H. and Stolterman, E. (2012). Digital form and materiality: Propositions for a new approach to interaction design research. In Proceedings of the 7th Nordic Conference on Human-Computer Interaction: Making Sense Through Design, NordiCHI '12, page 645-654, New York, NY, USA. Association for Computing Machinery.

[Kemp et al., 2010] Kemp, A. H., Quintana, D. S., Gray, M. A., Felmingham, K. L., Brown, K., and Gatt, J. M. (2010). Impact of depression and antidepressant treatment on heart rate variability: A review and meta-analysis. Biological Psychiatry, 67(11):1067-1074. 
[Khut, 2016] Khut, G. (2016). Designing biofeedback artworks for relaxation. In Proceedings of the $2016 \mathrm{CHI}$ Conference Extended Abstracts on Human Factors in Computing Systems, CHI EA '16, page 3859-3862, New York, NY, USA. Association for Computing Machinery.

[Khut, 2006] Khut, G. P. (2006). Development and Evaluation of ParticipantCentred Biofeedback Artworks. PhD thesis, School of Communication Arts, University of Western Sydney.

[Krasteva et al., 2015] Krasteva, V., Jekova, I., Leber, R., Schmid, R., and Abächerli, R. (2015). Superiority of classification tree versus cluster, fuzzy and discriminant models in a heartbeat classification system. PLOS ONE, 10(10):e0140123.

[Krauss, 1987] Krauss, K. E. (1987). The effects of deep pressure touch on anxiety. The American journal of occupational therapy : official publication of the American Occupational Therapy Association, 41 6:366-73.

[Kuznetsov and Paulos, 2010] Kuznetsov, S. and Paulos, E. (2010). Rise of the expert amateur: Diy projects, communities, and cultures. In Proceedings of the 6th Nordic Conference on Human-Computer Interaction: Extending Boundaries, NordiCHI '10, page 295-304, New York, NY, USA. Association for Computing Machinery.

[Lang et al., 2012] Lang, R., O'Reilly, M., Healy, O., Rispoli, M., Lydon, H., Streusand, W., Davis, T., Kang, S., Sigafoos, J., Lancioni, G., Didden, R., and Giesbers, S. (2012). Sensory integration therapy for autism spectrum disorders: A systematic review. Research in Autism Spectrum Disorders, 6(3):1004 $-1018$.

[Lazar et al., 2018] Lazar, A., Feuston, J. L., Edasis, C., and Piper, A. M. (2018). Making as expression: Informing design with people with complex communication needs through art therapy. In Proceedings of the $2018 \mathrm{CHI}$ Conference on Human Factors in Computing Systems, CHI '18, page 1-16, New York, NY, USA. Association for Computing Machinery.

[Lesobre, Vanessa, 2019] Lesobre, Vanessa (2019). Un air de flûte. https://fr. ulule. com/un-air-de-flute/. Online; accessed 29 March 2020. 
[Lindström et al., 2006] Lindström, M., Ståhl, A., Höök, K., Sundström, P., Laaksolathi, J., Combetto, M., Taylor, A., and Bresin, R. (2006). Affective diary: Designing for bodily expressiveness and self-reflection. In $\mathrm{CHI}$ '06 Extended $A b$ stracts on Human Factors in Computing Systems, CHI EA '06, page 1037-1042, New York, NY, USA. Association for Computing Machinery.

[Lipton et al., 2015] Lipton, Z. C., Berkowitz, J., and Elkan, C. (2015). A critical review of recurrent neural networks for sequence learning. ArXiv CoRR, abs/1506.00019.

[Liu et al., 2019] Liu, F., Esparza, M., Pavlovskaia, M., Kaufman, G., Dabbish, L., and Monroy-Hernández, A. (2019). Animo: Sharing biosignals on a smartwatch for lightweight social connection. Proc. ACM Interact. Mob. Wearable Ubiquitous Technol., 3(1).

[Loke and Schiphorst, 2018] Loke, L. and Schiphorst, T. (2018). The somatic turn in human-computer interaction. Interactions, 25(5):54-5863.

[Losinski et al., 2016] Losinski, M., Sanders, S. A., and Wiseman, N. M. (2016). Examining the use of deep touch pressure to improve the educational performance of students with disabilities: A meta-analysis. Research and Practice for Persons with Severe Disabilities, 41(1):3-18.

[Lucero et al., 2019] Lucero, A., Desjardins, A., Neustaedter, C., Höök, K., Hassenzahl, M., and Cecchinato, M. E. (2019). A sample of one: First-person research methods in $\mathrm{HCl}$. In Companion Publication of the 2019 on Designing Interactive Systems Conference 2019 Companion, DIS '19 Companion, page 385-388, New York, NY, USA. Association for Computing Machinery.

[Lukoševičius and Jaeger, 2009] Lukoševičius, M. and Jaeger, H. (2009). Reservoir computing approaches to recurrent neural network training. Computer Science Review, 3(3):127-149.

[MacLean et al., 2013] MacLean, D., Roseway, A., and Czerwinski, M. (2013). Moodwings: A wearable biofeedback device for real-time stress intervention. In Proceedings of the 6th International Conference on PErvasive Technologies Related to Assistive Environments, PETRA '13, New York, NY, USA. Association for Computing Machinery. 
[Maitland and Chalmers, 2010] Maitland, J. and Chalmers, M. (2010). Selfmonitoring, self-awareness, and self-determination in cardiac rehabilitation. In Proceedings of the SIGCHI Conference on Human Factors in Computing Systems, CHI '10, page 1213-1222, New York, NY, USA. Association for Computing Machinery.

[Marshall et al., 2019] Marshall, J., Benford, S., Byrne, R., and Tennent, P. (2019). Sensory alignment in immersive entertainment. In Proceedings of the 2019 CHI Conference on Human Factors in Computing Systems, CHI '19, page 1-13, New York, NY, USA. Association for Computing Machinery.

[Marshall et al., 2011] Marshall, J., Rowland, D., Rennick Egglestone, S., Benford, S., Walker, B., and McAuley, D. (2011). Breath control of amusement rides. In Proceedings of the SIGCHI Conference on Human Factors in Computing Systems, CHI '11, page 73-82, New York, NY, USA. Association for Computing Machinery.

[Massaroni et al., 2019] Massaroni, C., Nicolò, A., Presti, D. L., Sacchetti, M., Silvestri, S., and Schena, E. (2019). Contact-based methods for measuring respiratory rate. Sensors, 19(4):908.

[Matthews and Doherty, 2011] Matthews, M. and Doherty, G. (2011). In the mood: Engaging teenagers in psychotherapy using mobile phones. In Proceedings of the SIGCHI Conference on Human Factors in Computing Systems, CHI '11, page 2947-2956, New York, NY, USA. Association for Computing Machinery.

[Matthews et al., 2008] Matthews, M., Doherty, G., Sharry, J., and Fitzpatrick, C. (2008). Mobile phone mood charting for adolescents. British Journal of Guidance \& Counselling, 36(2):113-129.

[Matthews et al., 2015] Matthews, M., Voida, S., Abdullah, S., Doherty, G., Choudhury, T., Im, S., and Gay, G. (2015). In situ design for mental illness: Considering the pathology of bipolar disorder in mhealth design. In Proceedings of the 17th International Conference on Human-Computer Interaction with Mobile Devices and Services, MobileHCI '15, page 86-97, New York, NY, USA. Association for Computing Machinery.

[Melillo et al., 2011] Melillo, P., Bracale, M., and Pecchia, L. (2011). Nonlinear heart rate variability features for real-life stress detection. case study: students 
under stress due to university examination. BioMedical Engineering OnLine, 10(1):96.

[Mellis, 2014] Mellis, D. A. (2014). Do-it-yourself fabrication of electronic devices. IEEE Pervasive Computing, 13(3):22-29.

[Merleau-Ponty, 2002] Merleau-Ponty, M. (2002). Phenomenology of Perception. Routledge, London, UK.

[Merrill et al., 2019] Merrill, N., Chuang, J., and Cheshire, C. (2019). Sensing is believing: What people think biosensors can reveal about thoughts and feelings. In Proceedings of the 2019 on Designing Interactive Systems Conference, DIS '19, page 413-420, New York, NY, USA. Association for Computing Machinery.

[Miri et al., 2018a] Miri, P., Uusberg, A., Culbertson, H., Flory, R., Uusberg, H., Gross, J. J., Marzullo, K., and Isbister, K. (2018a). Emotion regulation in the wild: Introducing wehab system architecture. In Extended Abstracts of the 2018 CHI Conference on Human Factors in Computing Systems, CHI EA '18, page 1-6, New York, NY, USA. Association for Computing Machinery.

[Miri et al., 2018b] Miri, P., Uusberg, A., Culbertson, H., Flory, R., Uusberg, H., Gross, J. J., Marzullo, K., and Isbister, K. (2018b). Emotion regulation in the wild: The wehab approach. Technical report, University of California, Santa Cruz. https://www.soe.ucsc.edu/sites/default/files/technicalreports/ UCSC-SOE-18-04.pdf.

[Moraiti et al., 2015] Moraiti, A., Vanden Abeele, V., Vanroye, E., and Geurts, L. (2015). Empowering occupational therapists with a diy-toolkit for smart soft objects. In Proceedings of the Ninth International Conference on Tangible, Embedded, and Embodied Interaction, TEl '15, page 387-394, New York, NY, USA. Association for Computing Machinery.

[Morrill et al., 2019] Morrill, J., Kormilitzin, A., Nevado-Holgado, A., Swaminathan, S., Howison, S., and Lyons, T. (2019). The signature-based model for early detection of sepsis from electronic health records in the intensive care unit. In 2019 Computing in Cardiology Conference (CinC). Computing in Cardiology.

[Nakagaki et al., 2016] Nakagaki, K., Vink, L., Counts, J., Windham, D., Leithinger, D., Follmer, S., and Ishii, H. (2016). Materiable: Rendering dynamic material 
properties in response to direct physical touch with shape changing interfaces. In Proceedings of the $2016 \mathrm{CHI}$ Conference on Human Factors in Computing Systems, CHI '16, page 2764-2772, New York, NY, USA. Association for Computing Machinery.

[Nicolas-Alonso and Gomez-Gil, 2012] Nicolas-Alonso, L. F. and Gomez-Gil, J. (2012). Brain computer interfaces, a review. Sensors, 12(2):1211-1279.

[Norton et al., 2012] Norton, M. I., Mochon, D., and Ariely, D. (2012). The IKEA effect: When labor leads to love. Journal of Consumer Psychology, 22(3):453460 .

[Ortín et al., 2019] Ortín, S., Soriano, M. C., Alfaras, M., and Mirasso, C. R. (2019). Automated real-time method for ventricular heartbeat classification. Computer Methods and Programs in Biomedicine, 169:1-8.

[Pan and Tompkins, 1985] Pan, J. and Tompkins, W. J. (1985). A real-time QRS detection algorithm. IEEE Transactions on Biomedical Engineering, BME32(3):230-236.

[Paredes et al., 2018] Paredes, P. E., Zhou, Y., Hamdan, N. A.-H., Balters, S., Murnane, E., Ju, W., and Landay, J. A. (2018). Just breathe: In-car interventions for guided slow breathing. Proc. ACM Interact. Mob. Wearable Ubiquitous Technol., 2(1).

[Pedregosa et al., 2011] Pedregosa, F., Varoquaux, G., Gramfort, A., Michel, V., Thirion, B., Grisel, O., Blondel, M., Prettenhofer, P., Weiss, R., Dubourg, V., Vanderplas, J., Passos, A., Cournapeau, D., Brucher, M., Perrot, M., and Duchesnay, E. (2011). Scikit-learn: Machine learning in Python. Journal of Machine Learning Research, 12:2825-2830.

[Picard, 1997] Picard, R. W. (1997). Affective Computing. MIT Press, Cambridge, MA, USA.

[PLUX S.A., 2017] PLUX S.A. (2017). biosignalsplux. http://www. biosignalsplux.com.

[PLUX S.A., 2018] PLUX S.A. (2018). OpenSignals. http://www.bitalino.com/ software. 
[PLUX S.A., 2020] PLUX S.A. (2020). biosignalsnotebooks. https://github. com/biosignalsplux/biosignalsnotebooks.

[PLUX S.A. and IRCAM, 2017] PLUX S.A. and IRCAM (2017). BITalino R-IoT. https://bitalino.com/products/r-iot-2.

[Puckette, 1996] Puckette, M. (1996). Pure data: another integrated computer music environment. Proceedings of the second intercollege computer music concerts, pages 37-41.

[PureData, 2020] PureData (2020). Puredata (stable release). http://www. puredata.info.

[Rafael Lozano-Hemmer, 2018] Rafael Lozano-Hemmer (2018). Pulse Park. https://www. lozano-hemmer.com/pulse_park.php.

[Ramos et al., 2018] Ramos, G., Alfaras, M., and Gamboa, H. (2018). Real-time approach to HRV analysis. In Proceedings of the 11th International Joint Conference on Biomedical Engineering Systems and Technologies. SCITEPRESS Science and Technology Publications.

[Ramos et al., 2020] Ramos, G., Vaz, J. R., Mendonça, G. V., Pezarat-Correia, P., Rodrigues, J., Alfaras, M., and Gamboa, H. (2020). Fatigue evaluation through machine learning and a global fatigue descriptor. Journal of Healthcare Engineering, 2020:1-18.

[Razzaque et al., 2001] Razzaque, S., Kohn, Z., and Whitton, M. C. (2001). Redirected Walking. In Eurographics 2001 - Short Presentations. Eurographics Association.

[Reas and Fry, 2003] Reas, C. and Fry, B. (2003). Processing: A learning environment for creating interactive web graphics. In ACM SIGGRAPH 2003 Web Graphics, SIGGRAPH '03, page 1, New York, NY, USA. Association for Computing Machinery.

[Rehua (derivative) et al., 2014] Rehua (derivative), van Helvete, H., and Wikimedia Commons (2014). File:ekg complex en.svg - wikimedia commons, the free media repository. [Online; accessed 20-June-2020]. 
[Repetto et al., 2017] Repetto, C., Sas, C., Botella, C., and Riva, G. (2017). Affectech: Personal technologies for affective health. In The 15th European Congress of Psychology, 2017.

[Reyna et al., 2020] Reyna, M. A., Josef, C. S., Jeter, R., Shashikumar, S. P., Westover, M. B., Nemati, S., Clifford, G. D., and Sharma, A. (2020). Early prediction of sepsis from clinical data. Critical Care Medicine, 48(2):210-217.

[Rocchesso et al., 2019] Rocchesso, D., Monache, S. D., and Barrass, S. (2019). Interaction by ear. International Journal of Human-Computer Studies, 131:152159.

[Rodan and Tiňo, 2011] Rodan, A. and Tiňo, P. (2011). Minimum Complexity Echo State Network. IEEE Transactions on Neural Networks, 22(1):131-144.

[Rozzi et al., 1999] Rozzi, S. L., Lephart, S. M., and Fu, F. H. (1999). Effects of muscular fatigue on knee joint laxity and neuromuscular characteristics of male and female athletes. Journal of athletic training, 34(2):106.

[Russell, 1980] Russell, J. A. (1980). A circumplex model of affect. Journal of Personality and Social Psychology, 39(6):1161-1178.

[Samsung, 2015] Samsung (2015). Gear S2. https://www.samsung.com/ wearables/gear-s2/features.

[Sanches et al., 2019a] Sanches, P., Höök, K., Sas, C., and Ståhl, A. (2019a). Ambiguity as a resource to inform proto-practices: The case of skin conductance. ACM Trans. Comput.-Hum. Interact., 26(4).

[Sanches et al., 2010] Sanches, P., Höök, K., Vaara, E., Weymann, C., Bylund, M., Ferreira, P., Peira, N., and Sjölinder, M. (2010). Mind the body! designing a mobile stress management application encouraging personal reflection. In Proceedings of the 8th ACM Conference on Designing Interactive Systems, DIS '10, page 47-56, New York, NY, USA. Association for Computing Machinery.

[Sanches et al., 2019b] Sanches, P., Janson, A., Karpashevich, P., Nadal, C., Qu, C., Daudén Roquet, C., Umair, M., Windlin, C., Doherty, G., Höök, K., and Sas, C. (2019b). HCl and affective health: Taking stock of a decade of studies and charting future research directions. In Proceedings of the $2019 \mathrm{CHI}$ 
Conference on Human Factors in Computing Systems, CHI '19, New York, NY, USA. Association for Computing Machinery.

[Sas et al., 2020] Sas, C., Höök, K., Doherty, G., Sanches, P., Leufkens, T., and Westerink, J. (2020). Mental wellbeing: Future agenda drawing from design, $\mathrm{HCl}$ and big data. In Companion Publication of the 2020 ACM Designing Interactive Systems Conference, DIS' 20 Companion, page 425-428, New York, NY, USA. Association for Computing Machinery.

[Sas and Neustaedter, 2017] Sas, C. and Neustaedter, C. (2017). Exploring diy practices of complex home technologies. ACM Trans. Comput.-Hum. Interact., 24(2).

[Sas and Zhang, 2010a] Sas, C. and Zhang, C. (2010a). Do emotions matter in creative design? In Proceedings of the 8th ACM Conference on Designing Interactive Systems, DIS '10, page 372-375, New York, NY, USA. Association for Computing Machinery.

[Sas and Zhang, 2010b] Sas, C. and Zhang, C. (2010b). Investigating emotions in creative design. In Proceedings of the 1st DESIRE Network Conference on Creativity and Innovation in Design, DESIRE '10, page 138-149, Lancaster, GBR. Desire Network.

[Schmeder et al., 2010] Schmeder, A., Freed, A., and Wessel, D. (2010). Best practices for open sound control. In Linux Audio Conference, Utrecht, NL.

[schraefel et al., 2019] schraefel, m. c., Bateman, S., Friday, A., and Andres, J. (2019). The uncomfortable workshop: Exploring discomfort design for wellbeing and sustainability. In Adjunct Proceedings of the 2019 ACM International Joint Conference on Pervasive and Ubiquitous Computing and Proceedings of the 2019 ACM International Symposium on Wearable Computers, UbiComp/ISWC '19 Adjunct, page 1083-1086, New York, NY, USA. Association for Computing Machinery.

[Semmlow and Griffel, 2014] Semmlow, J. L. and Griffel, B. (2014). Biosignal and Medical Image Processing, Third Edition. CRC Press.

[Shelley et al., 2001] Shelley, K., Shelley, S., and Lake, C. (2001). Pulse oximeter waveform: photoelectric plethysmography. Clinical monitoring, pages 420-428. 
[Shusterman, 2012] Shusterman, R. (2012). Thinking through the Body. Cambridge University Press.

[Singer et al., 2016] Singer, M., Deutschman, C. S., Seymour, C. W., ShankarHari, M., Annane, D., Bauer, M., Bellomo, R., Bernard, G. R., Chiche, J.-D., Coopersmith, C. M., Hotchkiss, R. S., Levy, M. M., Marshall, J. C., Martin, G. S., Opal, S. M., Rubenfeld, G. D., van der Poll, T., Vincent, J.-L., and Angus, D. C. (2016). The third international consensus definitions for sepsis and septic shock (sepsis-3). JAMA, 315(8):801.

[Singh et al., 2019] Singh, J., Oshiro, K., Krishnan, R., Sato, M., Ohkuma, T., and Kato, N. (2019). Utilizing informative missingness for early prediction of sepsis. In 2019 Computing in Cardiology Conference (CinC). Computing in Cardiology.

[Smith and Koteen, 2019] Smith, N. S. and Koteen, D. (2019). Caught Falling: The Confluence of Contact Improvisation, Nancy Stark Smith, and Other Moving Ideas. Contact Quarterly.

[Solsona Belenguer, 2015] Solsona Belenguer, J. (2015). Engineering through Designerly Conversations with the Digital Material: The Approach, the Tools and the Design Space. PhD thesis, KTH School of Computer Science and Communication, Stockholm, Sweden.

[Solsona Belenguer et al., 2012] Solsona Belenguer, J., Lundén, M., Laaksolhati, J., and Sundström, P. (2012). Immaterial materials: Designing with radio. In Proceedings of the Sixth International Conference on Tangible, Embedded and Embodied Interaction, TEI '12, page 205-212, New York, NY, USA. Association for Computing Machinery.

[Soriano et al., 2015] Soriano, M. C., Brunner, D., an, M. E.-M., Mirasso, C. R., and Fischer, I. (2015). Minimal approach to neuro-inspired information processing. Frontiers in Computational Neuroscience, 9.

[Squeasewear, 2014] Squeasewear (2014). Squease. https://www. squeasewear. com.

[Ståhl and Höök, 2008] Ståhl, A. and Höök, K. (2008). Reflecting on the design process of the affective diary. In Proceedings of the 5th Nordic Conference on 
Human-Computer Interaction: Building Bridges, NordiCHI '08, page 559-564, New York, NY, USA. Association for Computing Machinery.

[Ståhl et al., 2016] Ståhl, A., Jonsson, M., Mercurio, J., Karlsson, A., Höök, K., and Banka Johnson, E.-C. (2016). The soma mat and breathing light. In Proceedings of the $2016 \mathrm{CHI}$ Conference Extended Abstracts on Human Factors in Computing Systems, CHI EA '16, page 305-308, New York, NY, USA. Association for Computing Machinery.

[Sundström et al., 2011] Sundström, P., Taylor, A., Grufberg, K., Wirström, N., Solsona Belenguer, J., and Lundén, M. (2011). Inspirational bits: Towards a shared understanding of the digital material. In Proceedings of the SIGCHI Conference on Human Factors in Computing Systems, CHI '11, page 1561-1570, New York, NY, USA. Association for Computing Machinery.

[Tangible Media. MIT Media Lab, 2016] Tangible Media. MIT Media Lab (2016). Project materiable. http://tangible.media.mit.edu/project/materiable.

[Task Force of the European Society of Cardiology, 1996] Task Force of the European Society of Cardiology (1996). Heart rate variability. Circulation, 93(5):1043-1065.

[TEAM ITN, 2016] TEAM ITN (2016). TEAM ITN: Technology Enabled Mental Health. https://www. team-itn. eu. Accessed: 2020-06-04.

[Teh et al., 2008] Teh, J. K. S., Cheok, A. D., Peiris, R. L., Choi, Y., Thuong, V., and Lai, S. (2008). Huggy pajama: A mobile parent and child hugging communication system. In Proceedings of the 7th International Conference on Interaction Design and Children, IDC '08, page 250-257, New York, NY, USA. Association for Computing Machinery.

[Tennent et al., 2020] Tennent, P., Marshall, J., Tsaknaki, V., Windlin, C., Höök, K., and Alfaras, M. (2020). Soma design and sensory misalignment. In Proceedings of the 2020 CHI Conference on Human Factors in Computing Systems, CHI '20, page 1-12, New York, NY, USA. Association for Computing Machinery.

[Tennent et al., 2011] Tennent, P., Rowland, D., Marshall, J., Egglestone, S. R., Harrison, A., Jaime, Z., Walker, B., and Benford, S. (2011). Breathalising games: Understanding the potential of breath control in game interfaces. In Proceedings 
of the 8th International Conference on Advances in Computer Entertainment Technology, ACE '11, New York, NY, USA. Association for Computing Machinery.

[Tortella-Feliu et al., 2010] Tortella-Feliu, M., Botella, C., Llabrés, J., BretónLópez, J. M., del Amo, A. R., Baños, R. M., and Gelabert, J. M. (2010). Virtual reality versus computer-aided exposure treatments for fear of flying. Behavior Modification, 35(1):3-30.

[Tsaknaki et al., 2019] Tsaknaki, V., Balaam, M., Ståhl, A., Sanches, P., Windlin, C., Karpashevich, P., and Höök, K. (2019). Teaching soma design. In Proceedings of the 2019 on Designing Interactive Systems Conference, DIS '19, page 1237-1249, New York, NY, USA. Association for Computing Machinery.

[Tware, 2015] Tware (2015). TJacket. https://www. mytjacket.com/.

[Ubicom Mental Health, 2015] Ubicom Mental Health (2015). Ubiquitous mental health support systems for managing long-term mental health illness. https: //cordis . europa.eu/project/id/302530/reporting. Accessed: 2019-05-10.

[Umair et al., 2019a] Umair, M., Alfaras, M., Gamboa, H., and Sas, C. (2019a). Experiencing discomfort: Designing for affect from first-person perspective. In Adjunct Proceedings of the 2019 ACM International Joint Conference on Pervasive and Ubiquitous Computing and Proceedings of the 2019 ACM International Symposium on Wearable Computers, UbiComp/ISWC '19 Adjunct, page 1093-1096, New York, NY, USA. Association for Computing Machinery.

[Umair et al., 2018] Umair, M., Latif, M. H., and Sas, C. (2018). Dynamic displays at wrist for real time visualization of affective data. In Proceedings of the 2018 ACM Conference Companion Publication on Designing Interactive Systems, DIS '18 Companion, page 201-205, New York, NY, USA. Association for Computing Machinery.

[Umair et al., 2020] Umair, M., Sas, C., and Alfaras, M. (2020). ThermoPixels: Toolkit for personalizing arousal-based interfaces through hybrid crafting. In Proceedings of the 2020 ACM Designing Interactive Systems Conference, DIS '20, page 1017-1032, New York, NY, USA. Association for Computing Machinery.

[Umair et al., 2019b] Umair, M., Sas, C., and Latif, M. H. (2019b). Towards affective chronometry: Exploring smart materials and actuators for real-time 
representations of changes in arousal. In Proceedings of the 2019 on Designing Interactive Systems Conference, DIS '19, pages 1479-1494, New York, NY, USA. ACM.

[Vallgårda et al., 2017] Vallgårda, A., Boer, L., Tsaknaki, V., and Svanæs, D. (2017). Material programming. Interactions, 24(3):36-41.

[van de Ven et al., 2012] van de Ven, P., Henriques, M. R., Hoogendoorn, M., Klein, M., McGovern, E., Nelson, J., Silva, H., and Tousset, E. (2012). A mobile system for treatment of depression. In Proceedings of the 2nd International Workshop on Computing Paradigms for Mental Health - Volume 1: MindCare, (BIOSTEC 2012), pages 47-58. INSTICC, SciTePress.

[VanderPlas, 2018] VanderPlas, J. T. (2018). Understanding the lomb-scargle periodogram. The Astrophysical Journal Supplement Series, 236(1):16.

[Varandas et al., 2020] Varandas, R., Ramos, G., Mrotzeck, K., Gamboa, P., and Gamboa, H. (2020). Biosignals Notebooks: An Innovative Approach for Biosignals Experience Sharing. In INTED2020 Proceedings. IATED.

[Vaucelle et al., 2009] Vaucelle, C., Bonanni, L., and Ishii, H. (2009). Design of haptic interfaces for therapy. In Proceedings of the SIGCHI Conference on Human Factors in Computing Systems, CHI '09, page 467-470, New York, NY, USA. Association for Computing Machinery.

[Wang et al., 2017] Wang, Y., Luo, S., Lu, Y., Gong, H., Zhou, Y., Liu, S., and Hansen, P. (2017). Animskin: Fabricating epidermis with interactive, functional and aesthetic color animation. In Proceedings of the 2017 Conference on Designing Interactive Systems, DIS '17, page 397-401, New York, NY, USA. Association for Computing Machinery.

[Wiberg, 2013] Wiberg, M. (2013). Methodology for materiality: interaction design research through a material lens. Personal and Ubiquitous Computing, 18(3):625-636.

[Wilde et al., 2017] Wilde, D., Vallgårda, A., and Tomico, O. (2017). Embodied design ideation methods: Analysing the power of estrangement. In Proceedings of the 2017 CHI Conference on Human Factors in Computing Systems, CHI '17, page 5158-5170, New York, NY, USA. Association for Computing Machinery. 
[Windlin et al., 2019] Windlin, C., Ståhl, A., Sanches, P., Tsaknaki, V., Karpashevich, P., Balaam, M., and Höök, K. (2019). Soma Bits: Mediating technology to orchestrate bodily experiences. In Research Through Design RTD 2019, pages 19-22.

[World Health Organization, 2017] World Health Organization (2017). Depression and other common mental disorders: Global health estimates. Technical report, Geneva, $\mathrm{CH}$.

[Zucker et al., 2009] Zucker, T. L., Samuelson, K. W., Muench, F., Greenberg, M. A., and Gevirtz, R. N. (2009). The effects of respiratory sinus arrhythmia biofeedback on heart rate variability and posttraumatic stress disorder symptoms: A pilot study. Applied Psychophysiology and Biofeedback, 34(2):135-143. 